\title{
Lorentzian geometrical structures with global time, Gravity and Electrodynamics
}

This is a preprint of the following work: Arkady Poliakovsky, Lorentzian Geometrical Structures with Global Time, Gravity and Electrodynamics, 2023, Springer Cham, reproduced with permission of Springer Nature Switzerland AG 2023. The final authenticated version is available online at: https://doi.org/10.1007/978-3-031-23762-1

\author{
ARKADY POLIAKOVsky ${ }^{1}$ \\ Department of Mathematics, Ben Gurion University of the Negev, \\ P.O.B. 653, Be'er Sheva 84105, Israel
}

\begin{abstract}
We investigate Lorentzian structures in the four-dimensional space-time, supplemented either by a covector field of the time-direction or by a scalar field of the global time. Furthermore, we propose a new metrizable model of the gravity. In contrast to the usual Theory of General Relativity where all ten components of the symmetric pseudo-metric are independent variables, the model of gravity presented here essentially depends only on a single four-covector field, and is restricted to have only three-independent components. However, we prove that the Gravitational field, governed by the proposed model and generated by some massive body, resting and spherically symmetric in some coordinate system, is given by a pseudo-metric $\left\{K_{m n}\right\}_{m, n=0,1,2,3}$, which coincides with the well known Schwarzschild metric from General Relativity. The Maxwell equations and Electrodynamics are also investigated in the framework of the proposed model.
\end{abstract}

${ }^{1}$ E-mails: poliakov@math.bgu.ac.il, arkady.pol@gmail.com 
In particular, we derive the covariant formulation of Electrodynamics of moving dielectrics and para/diamagnetic mediums.

\section{Preliminary introduction}

In the classical theories of Special and General Relativity the inertia and the gravity are described by certain pseudo-metric of signature $\{1,-1,-1,-1\}$ in the four-dimensional space-time. On the other hand, in the framework of the Newton-Cartan Theory (see [1], [2], [3], [4] [5], [6]) the geometry of the space-time is (incompletely) described by the two-times contravariant symmetric degenerate tensor $\left\{h^{m n}\right\}_{m, n=0,1,2,3}$ of signature $\{0,1,1,1\}$ and a covector $\left(w_{0}, w_{1}, w_{2}, w_{3}\right)$ of time direction, everywhere non-vanishing and satisfying

$$
\sum_{j=0}^{3} h^{m j} w_{j}=0 \quad \forall m=0,1,2,3 .
$$

Moreover, in the case that there exists a scalar field $\tau$ satisfying

$$
w_{m}=\frac{\partial \tau}{\partial x^{m}} \quad \forall m=0,1,2,3,
$$

this field can serve as a global time in $\mathbb{R}^{4}$. In this paper we unify both these approaches and build the model, completely describing the geometry and the gravity in $\mathbb{R}^{4}$, which includes both the pseudometric and the global time scalar field (or more generally the covector of the time direction). One of the goal of the paper was to unify the Relativistic and the Non-Relativistic approaches to the study of the space-time.

We postulate that all real physical processes appear in some valid pseudo-metric $\left\{K^{m n}\right\}_{m, n=0,1,2,3}$, describing the generalized gravity field, weakly correlated with some covector of time direction $\left(w_{0}, w_{1}, w_{2}, w_{3}\right)$ (see definitions in the sequent section 2$)$. Furthermore, we distinguish two types of generalized gravity. The first type is the fictitious gravity which we call inertia. This type of gravity depends only on the flat geometry of empty space-time via the choice of specific coordinate system and it is independent on the surrounding real matter consisting of gravitational masses or other real physical fields. The second type of the gravity is the genuine (real) gravity, which depends essentially on the real physical matter, especially on gravitational masses. We assume that this type of gravity vanishes away from essential gravitational masses and strong real physical fields. Then we state the First Law of the Newton as the following:

- In the parts of the space-time where we observes the absence of genuine gravity, and in particular away from essential real physical bodies and fields, we have

$$
K^{m n}=J^{m n} \quad \forall m, n=0,1,2,3,
$$

and

$$
\left(w_{0}, w_{1}, w_{2}, w_{3}\right)=\left(\frac{\partial \varphi}{\partial x^{0}}, \frac{\partial \varphi}{\partial x^{1}}, \frac{\partial \varphi}{\partial x^{2}}, \frac{\partial \varphi}{\partial x^{3}}\right)
$$


where the strongly-correlating flat Minkowski's pseudo-metric $\left\{J^{m n}\right\}_{m, n=0,1,2,3}$ and the fixed scalar field $\varphi$, called kinematical global time, form the standard kinematical Lorentz's structure with global time on $\mathbb{R}^{4}$, as defined in the Definition 2.7 of the sequent section. In particular they assumed to satisfy, firstly the following eikonal-type equation

$$
\sum_{j=0}^{3} \sum_{m=0}^{3} J^{j m} \frac{\partial \varphi}{\partial x^{j}} \frac{\partial \varphi}{\partial x^{m}}=1
$$

and secondly

$$
\left\{\delta_{j}\left(\frac{\partial \varphi}{\partial x^{k}}\right)\right\}_{J}=0 \quad \forall k, j=0,1,2,3 .
$$

where by $\left\{\delta_{j}\left(\frac{\partial \varphi}{\partial x^{k}}\right)\right\}_{J}$ we denote the tensor of the covariant derivatives of the covector $\left(\frac{\partial \varphi}{\partial x^{0}}, \frac{\partial \varphi}{\partial x^{1}}, \frac{\partial \varphi}{\partial x^{2}}, \frac{\partial \varphi}{\partial x^{3}}\right)$ with respect to the pseudometric $J^{m n}$.

Then it easily can be derived that there exists some coordinate system where matrix $\left\{J^{m n}\right\}_{m, n=0,1,2,3}$ has a form of

$$
\begin{cases}J^{00}=1 & \\ J^{0 j}=J^{j 0}=0 & \forall j=1,2,3 \\ J^{j m}:=-\delta_{j m} & \forall j, m=1,2,3\end{cases}
$$

and at the same coordinate system the covector of time direction for the global time $\varphi$ has a form

$$
\left(\frac{\partial \varphi}{\partial x^{0}}, \frac{\partial \varphi}{\partial x^{1}}, \frac{\partial \varphi}{\partial x^{2}}, \frac{\partial \varphi}{\partial x^{3}}\right)\left(x^{0}, x^{1}, x^{2}, x^{3}\right)=(1,0,0,0) \quad \forall\left(x^{0}, x^{1}, x^{2}, x^{3}\right) \in \mathbb{R}^{4} .
$$

We call this particular system kinematically preferable and we show that it is unique, up to equivalence. Furthermore, we define the kinematical tensor of three-dimensional Geometry $\left\{\Theta^{m n}\right\}_{m, n=0,1,2,3}$, given by

$$
\Theta^{m n}:=\left(\sum_{j=0}^{3} J^{m j} \frac{\partial \varphi}{\partial x^{j}}\right)\left(\sum_{j=0}^{3} J^{n j} \frac{\partial \varphi}{\partial x^{j}}\right)-J^{m n} \quad \forall m, n=0,1,2,3,
$$

where

$$
\left(\sum_{j=0}^{3} J^{0 j} \frac{\partial \varphi}{\partial x^{j}}, \sum_{j=0}^{3} J^{1 j} \frac{\partial \varphi}{\partial x^{j}}, \sum_{j=0}^{3} J^{2 j} \frac{\partial \varphi}{\partial x^{j}}, \sum_{j=0}^{3} J^{3 j} \frac{\partial \varphi}{\partial x^{j}}\right)
$$

is the contravariant vector of inertia. Obviously, we have

$$
\sum_{j=0}^{3} \Theta^{m j} \frac{\partial \varphi}{\partial x^{j}}=0 \quad \forall m=0,1,2,3,
$$

(as in (1.1)), forming the standard Galilean structure. In particular, in the kinematically preferable coordinate system, where (1.7) and (1.8) holds we have

$$
\left\{\begin{array}{l}
\Theta^{00}=0 \\
\Theta^{0 j}=\Theta^{j 0}=0 \quad \forall j=1,2,3 \\
\Theta^{j m}:=\delta_{j m} \quad \forall j, m=1,2,3 .
\end{array}\right.
$$


Furthermore, given arbitrary coordinate system, it is called cartesian if in this system we have simultaneously (1.12) and (1.8) but, we do not necessary have (1.7). On the other hand, given arbitrary coordinate system, it is called Lorentzian if in this system we have (1.7) but, we do not necessary have (1.12) or (1.8). Finally, given arbitrary coordinate system, we call it inertial, if we can get it from kinematically preferable coordinate system by a linear transformation. In the sequence we prove, that we obtain a coordinate system which is simultaneously cartesian and inertial from another such system by Galilean transformations. On the other hand, we obtain a coordinate system which is Loretzian (and then also inertial) from another such system by Lorentz's transformations. The unique, up to equivalence, coordinate system which is simultaneously cartesian and Loretzian is a kinematically preferable coordinate system. In subsection 2.1.1 we define Pseudo-Lorentzian coordinate systems that generalize both cartesian and Lorentzian systems. We also find the group of transformation of such systems including, in particular as subgroups Lorentzian and Galilean transformations.

Furthermore, we describe our model of the gravity: given, an arbitrary dynamical four-covector of the dynamical time direction, $\left(w_{0}, w_{1}, w_{2}, w_{3}\right)$ (formally unrelated to the kinematical global time $\varphi$ ), which is weakly correlated with $\left\{J^{m n}\right\}_{m, n=0,1,2,3}$ and an arbitrary four-covector field $\left(S_{0}, S_{1}, S_{2}, S_{3}\right)$, which we call the four-covector of genuine gravity, consider the two-times covariant tensor $\left\{K_{m n}\right\}_{m, n=0,1,2,3}$ defined by:

$$
K_{j m}=\left(J_{j m}+w_{j} S_{m}+w_{m} S_{j}\right) \quad \forall 0 \leq j, m \leq 3,
$$

and assume that $\left(S_{0}, S_{1}, S_{2}, S_{3}\right)$ is such that $\left\{K_{m n}\right\}_{m, n=0,1,2,3}$ in (1.13) satisfies

$$
\operatorname{det}\left(\left\{K_{m n}\right\}_{m, n=0,1,2,3}\right)=\operatorname{det}\left(\left\{J_{m n}\right\}_{m, n=0,1,2,3}\right) .
$$

Then, one can prove that $\left\{K_{m n}\right\}_{m, n=0,1,2,3}$, is a valid pseudometric of signature $\{1,-1,-1,-1\}$, correlated with the time-direction $\left(w_{0}, w_{1}, w_{2}, w_{3}\right)$. Moreover, we call such a dynamical pseudometric $\left\{K_{m n}\right\}_{m, n=0,1,2,3}$, with time-direction $\left(w_{0}, w_{1}, w_{2}, w_{3}\right)$, correlated pseudometric with time-direction $\left(w_{0}, w_{1}, w_{2}, w_{3}\right)$, corresponding to the covector of genuine gravity $\left(S_{0}, S_{1}, S_{2}, S_{3}\right)$. In the case of the simplified approximating model we get

$$
\left(w_{0}, w_{1}, w_{2}, w_{3}\right) \approx\left(\frac{\partial \varphi}{\partial x^{0}}, \frac{\partial \varphi}{\partial x^{1}}, \frac{\partial \varphi}{\partial x^{2}}, \frac{\partial \varphi}{\partial x^{3}}\right),
$$

and (1.13) reeds as

$$
K_{j m}=\left(J_{j m}+\frac{\partial \varphi}{\partial x^{j}} S_{m}+\frac{\partial \varphi}{\partial x^{m}} S_{j}\right) \quad \forall 0 \leq j, m \leq 3,
$$

where $\varphi$ is the kinematical global time. Next for the dynamical time direction, $\left(w_{0}, w_{1}, w_{2}, w_{3}\right)$ and the covector of genuine gravity $\left(S_{0}, S_{1}, S_{2}, S_{3}\right)$ one can consider Proca-like Lagrangians, see in the sequel.

In the following sections we prove, in particular, that in the framework of our simplified model, the Gravitational field, generated by some massive body, resting and spherically symmetric in some 
$\underline{\text { cartesian }}$ and inertial coordinate system, is given by the pseudo-metric $\left\{K_{m n}\right\}_{m, n=0,1,2,3}$, such that there exists some curvilinear (non-cartesian) coordinate system in $\mathbb{R}^{4}$, where $\left\{K_{m n}\right\}_{m, n=0,1,2,3}$ coincides with the well known Schwarzschild metric from the General Relativity! In particular, all the optical effects that we find in the framework of our model coincide with the effects considered in the framework of General Relativity for the Schwarzschild metric. Finally, all the mechanical effects will be the same in the framework of our model like in the case of the General relativity for the Schwarzschild metric, provided that the time does not appear explicitly in this effects. Furthermore, we also prove that Gravitational field, governed by our model, generated by a general slowly (non-relativistically) moving massive matter in some cartesian coordinate system, can be well approximated, by the classical model of the Newtonian Gravity.

Note here about the following advantage of the presented model of gravity with respect to the usual Theory of General Relativity. The simplified model for the gravity depends only on fourcomponent field $\left(S_{0}, S_{1}, S_{2}, S_{3}\right)$, which is by (1.16) and (1.14) has only three independent components. Even the full model dependent only on $\left(S_{0}, S_{1}, S_{2}, S_{3}\right)$ and $\left(w_{0}, w_{1}, w_{2}, w_{3}\right)$, that is by (1.13) and (1.14) has only seven independent components. On the other hand, in the General Relativity the symmetric tensor $\left\{K_{m n}\right\}_{m, n=0,1,2,3}$ has all ten independent components that makes the corresponding system of partial differential equations to be much more complicated.

Finally, in section 9 we give the covariant formulation of the Electrodynamics of the moving dielectric and para/dia-magnetic continuum mediums in arbitrary dynamical pseudo-metric. The Lorentz's covariant theory of the moving para/dia-magnetic continuum mediums in the flat Lorentz's pseudo-metric was first introduced in [8] by H. Minkowski (1908). Here we formulate the generally covariant theory in the different alternative way, that suite to formulate it in a general pseudometric including the presence of the genuine gravity. Note that for the formulation of the concepts of polarization and magnetization and for the mathematical proofs we use in particular the theory of distributions (generalized functions).

The next section plays a role of comprehensive introduction. In the sequent sections we give more detailed description of our results. In the end of the paper including Appendix we give detailed prove of all mathematical statements.

\section{Basic definitions and statements of the main results}

\subsection{Generalized-Lorentz's structures with time-direction and global time}

Definition 2.1. We say that the generalized-Lorentz's structure on $\mathbb{R}^{4}$ is chosen, if $\mathbb{R}^{4}$ is equipped with symmetric non-degenerate two-times contravariant tensor field $\left\{K^{m n}\right\}_{m, n=0,1,2,3}$, such that the matrix $K^{m n}$ has one positive and three negative eigenvalues at every point in $\mathbb{R}^{4}$. Then, $\left\{K^{m n}\right\}_{m, n=0,1,2,3}$ is called a contravariant pseudo-metric on $\mathbb{R}^{4}$. Moreover, the inverse symmet- 
ric non-degenerate two-times covariant tensor field $\left\{K_{m n}\right\}_{m, n=0,1,2,3}$ which satisfies

$$
\sum_{k=0}^{3} K^{m k} K_{k n}=\left\{\begin{array}{lll}
1 & \text { if } & m=n \\
0 & \text { if } & m \neq n
\end{array} \quad \forall m, n=0,1,2,3,\right.
$$

is called a covariant pseudo-metric on $\mathbb{R}^{4}$ associated with $\left\{K^{m n}\right\}_{m, n=0,1,2,3}$.

Definition 2.2. We say that a direction of the global time on $\mathbb{R}^{4}$ is chosen, if $\mathbb{R}^{4}$ is equipped with a four-covector field $\left(w_{0}, w_{1}, w_{2}, w_{3}\right):=\left(w_{0}, w_{1}, w_{2}, w_{3}\right)\left(x^{0}, x^{1}, x^{2}, x^{3}\right)$, non-vanishing at every point in $\mathbb{R}^{4}$ (the last property is obviously independent on the choice of a coordinate system in $\mathbb{R}^{4}$ ). Then, chosen co-vector field $\left(w_{0}, w_{1}, w_{2}, w_{3}\right)$ is called the co-vector of the time-direction. Furthermore, we say that a scalar global time on $\mathbb{R}^{4}$ is chosen, if $\mathbb{R}^{4}$ is equipped with a covariant scalar field $\varphi:=\varphi\left(x^{0}, x^{1}, x^{2}, x^{3}\right)$ such that the four-covector field $\left(w_{0}, w_{1}, w_{2}, w_{3}\right)$, defined by

$$
w_{j}:=\frac{\partial \varphi}{\partial x^{j}} \quad \forall j=0,1,2,3,
$$

does not vanish at any point on $\mathbb{R}^{4}$. Then $\varphi$ is called a global time in $\mathbb{R}^{4}$ and $w_{j}:=w_{j}\left(x^{0}, x^{1}, x^{2}, x^{3}\right)$, given by $(2.2)$ is called the co-vector of the time-direction of the given global time $\varphi$.

Definition 2.3. We say that a contravariant pseudo-metric $\left\{K^{m n}\right\}_{m, n=0,1,2,3}$ and a covector of the time-direction $\left(w_{0}, w_{1}, w_{2}, w_{3}\right)$ are weakly correlated, if we have everywhere

$$
\sum_{j=0}^{3} \sum_{m=0}^{3} K^{j m} w_{j} w_{m}>0 \quad \forall\left(x^{0}, x^{1}, x^{2}, x^{3}\right) \in \mathbb{R}^{4} .
$$

In that case we say that generalized-Lorentz's structure with time-direction on $\mathbb{R}^{4}$ is chosen. Then, we define the contrvariant four-vector field of the potential of generalized-gravity $\left(v^{0}, v^{1}, v^{2}, v^{3}\right)$ by

$$
v^{m}:=\left(\sum_{j=0}^{3} \sum_{k=0}^{3} K^{j k} w_{j} w_{k}\right)^{-\frac{1}{2}}\left(\sum_{j=0}^{3} K^{m j} w_{j}\right) \quad \forall m=0,1,2,3,
$$

so that we have

$$
\sum_{j=0}^{3} \sum_{m=0}^{3} K_{j m} v^{j} v^{m}=1 \quad \forall\left(x^{0}, x^{1}, x^{2}, x^{3}\right) \in \mathbb{R}^{4} .
$$

Furthermore, we say that a contravariant pseudo-metric $\left\{K^{m n}\right\}_{m, n=0,1,2,3}$ and a scalar global time $\varphi$ are strongly correlated on $\mathbb{R}^{4}$ if $\varphi$ satisfies the following eikonal-type equation in pseudo-metric $\left\{K^{m n}\right\}_{m, n=0,1,2,3}$ :

$$
\sum_{j=0}^{3} \sum_{m=0}^{3} K^{j m} \frac{\partial \varphi}{\partial x^{j}} \frac{\partial \varphi}{\partial x^{m}}=1 \quad \forall\left(x^{0}, x^{1}, x^{2}, x^{3}\right) \in \mathbb{R}^{4} .
$$

In that case we say that generalized-Lorentz's structure with global time on $\mathbb{R}^{4}$ is chosen. Moreover, in the later case we rewrite the definition of the contrvariant four-vector field of the potential of generalized-gravity $\left(v^{0}, v^{1}, v^{2}, v^{3}\right)$ in $(2.4)$ as:

$$
v^{m}:=\sum_{j=0}^{3} K^{m j} \frac{\partial \varphi}{\partial x^{j}} \quad \forall m=0,1,2,3 .
$$


Then we prove the following:

Proposition 2.1. Given a contravariant pseudo-metric $\left\{K^{m n}\right\}_{m, n=0,1,2,3}$ on $\mathbb{R}^{4}$, weakly correlated with a covector of the time-direction $\left(w_{0}, w_{1}, w_{2}, w_{3}\right)$, define the two-times contravariant symmetric tensor field $\left\{\Lambda^{m n}\right\}_{m, n=0,1,2,3}$ given by

$$
\Lambda^{j m}:=v^{j} v^{m}-K^{j m} \quad \forall j, m=0,1,2,3,
$$

with $\left(v^{0}, v^{1}, v^{2}, v^{3}\right)$ defined by (2.4). Then, the matrix $\Lambda^{m n}$ has one vanishing and three positive eigenvalues at every point in $\mathbb{R}^{4}$, and we call $\left\{\Lambda^{m n}\right\}_{m, n=0,1,2,3}$ the contravariant tensor of threedimensional Geometry on $\mathbb{R}^{4}$. Moreover, we have

$$
\sum_{j=0}^{3} v^{j} w_{j}=\left(\sum_{j=0}^{3} \sum_{k=0}^{3} K^{j k} w_{j} w_{k}\right)^{\frac{1}{2}}
$$

and

$$
\sum_{j=0}^{3} \Lambda^{m j} w_{j}=0 \quad \forall m=0,1,2,3 .
$$

Corollary 2.1. Given a contravariant pseudo-metric $\left\{K^{m n}\right\}_{m, n=0,1,2,3}$ on $\mathbb{R}^{4}$, strongly correlated with a scalar global time $\varphi$, define the contravariant tensor field $\left\{\Lambda^{m n}\right\}_{m, n=0,1,2,3}$, given by

$$
\Lambda^{j m}:=v^{j} v^{m}-K^{j m} \quad \forall j, m=0,1,2,3,
$$

with $\left(v^{0}, v^{1}, v^{2}, v^{3}\right)$, defined by (2.7). Then, the matrix $\Lambda^{m n}$ has one vanishing and three positive eigenvalues at every point in $\mathbb{R}^{4}$. Moreover, we have

$$
\sum_{j=0}^{3} v^{j} \frac{\partial \varphi}{\partial x^{j}}=1
$$

and

$$
\sum_{j=0}^{3} \Lambda^{m j} \frac{\partial \varphi}{\partial x^{j}}=0 \quad \forall m=0,1,2,3 .
$$

Here, it is important to note that in the framework of the Newton-Cartan Theory we have the following definition (see [1], [2], [3], [4] [5], [6]):

Definition 2.4. The Galilean structure in $\mathbb{R}^{4}$ consists of a fixed two-times contravariant symmetric tensor $\left\{\Lambda^{m n}\right\}_{m, n=0,1,2,3}$ in $\mathbb{R}^{4}$, such that the matrix $\Lambda^{m n}$ has one vanishing and three positive eigenvalues at every point in $\mathbb{R}^{4}$, and a fixed covector $\left(w_{0}, w_{1}, w_{2}, w_{3}\right)$, non-vanishing at every every point in $\mathbb{R}^{4}$ and such that

$$
\sum_{j=0}^{3} \Lambda^{m j} w_{j}=0 \quad \forall m=0,1,2,3 \quad \forall\left(x^{0}, x^{1}, x^{2}, x^{3}\right) \in \mathbb{R}^{4} .
$$

Then, we prove the inverse to Proposition 2.1 statement: 
Proposition 2.2. Let $\left\{\Lambda^{m n}\right\}_{m, n=0,1,2,3}$ be a two-times contravariant symmetric tensor, such that the matrix $\Lambda^{m n}$ has one vanishing and three positive eigenvalues at every point in $\mathbb{R}^{4}$, and let $\left(w_{0}, w_{1}, w_{2}, w_{3}\right)$ be a covector, non-vanishing at every every point in $\mathbb{R}^{4}$ and such that

$$
\sum_{j=0}^{3} \Lambda^{m j} w_{j}=0 \quad \forall m=0,1,2,3 \quad \forall\left(x^{0}, x^{1}, x^{2}, x^{3}\right) \in \mathbb{R}^{4},
$$

that form together a Galileian structure. Next, given an arbitrary contravariant four-vector field $\left(v^{0}, v^{1}, v^{2}, v^{3}\right)$ satisfying the covariant relation

$$
\sum_{j=0}^{3} v^{j} w_{j}>0 \quad \forall\left(x^{0}, x^{1}, x^{2}, x^{3}\right) \in \mathbb{R}^{4},
$$

consider a contravariant symmetric tensor field $\left\{K^{m n}\right\}_{m, n=0,1,2,3}$, given by

$$
K^{m n}=v^{j} v^{m}-\Lambda^{j m} \quad \forall j, m=0,1,2,3 .
$$

Then, $\left\{K^{m n}\right\}_{m, n=0,1,2,3}$ is a valid contravariant pseudo-metric. Moreover, the generalized-Lorentz's structure, given by $\left\{K^{m n}\right\}_{m, n=0,1,2,3}$ is weakly correlated with the time direction $\left(w_{0}, w_{1}, w_{2}, w_{3}\right)$. Finally, we also have

$$
\left(\sum_{j=0}^{3} \sum_{k=0}^{3} K^{j m} w_{j} w_{k}\right)^{\frac{1}{2}}=\sum_{j=0}^{3} v^{j} w_{j} \quad \forall\left(x^{0}, x^{1}, x^{2}, x^{3}\right) \in \mathbb{R}^{4},
$$

and

$$
v^{m}=\left(\sum_{j=0}^{3} \sum_{k=0}^{3} K^{j m} w_{j} w_{k}\right)^{-\frac{1}{2}}\left(\sum_{j=0}^{3} K^{j m} w_{j}\right) \quad \forall m=0,1,2,3 \quad \forall\left(x^{0}, x^{1}, x^{2}, x^{3}\right) \in \mathbb{R}^{4},
$$

So, $\left\{\Lambda^{m n}\right\}_{m, n=0,1,2,3}$ is a contravariant tensor of three-dimensional Geometry and $\left(v^{0}, v^{1}, v^{2}, v^{3}\right)$ is a potential of generalized gravity, corresponding to the pseudo-metric $K^{m n}$ and the time-direction $\left(w_{0}, w_{1}, w_{2}, w_{3}\right)$.

Corollary 2.2. Let $\left\{\Lambda^{m n}\right\}_{m, n=0,1,2,3}$ be a two-times contravariant symmetric tensor, such that the matrix $\Lambda^{m n}$ has one vanishing and three positive eigenvalues at every point in $\mathbb{R}^{4}$, and let $\varphi$ be a covariant scalar field such that the four-covector field $\left(w_{0}, w_{1}, w_{2}, w_{3}\right)$, defined by

$$
w_{j}:=\frac{\partial \varphi}{\partial x^{j}} \quad \forall j=0,1,2,3,
$$

does not vanish at any point on $\mathbb{R}^{4}$ and such that

$$
\sum_{j=0}^{3} \Lambda^{m j} \frac{\partial \varphi}{\partial x^{j}}=0 \quad \forall m=0,1,2,3 \quad \forall\left(x^{0}, x^{1}, x^{2}, x^{3}\right) \in \mathbb{R}^{4} .
$$

Next, given an arbitrary contravariant four-vector field $\left(v^{0}, v^{1}, v^{2}, v^{3}\right)$ satisfying the covariant relation

$$
\sum_{j=0}^{3} v^{j} \frac{\partial \varphi}{\partial x^{j}}=1 \quad \forall\left(x^{0}, x^{1}, x^{2}, x^{3}\right) \in \mathbb{R}^{4},
$$


consider a contravariant symmetric tensor field $\left\{K^{m n}\right\}_{m, n=0,1,2,3}$, given by

$$
K^{m n}=v^{j} v^{m}-\Lambda^{j m} \quad \forall j, m=0,1,2,3 .
$$

Then, $\left\{K^{m n}\right\}_{m, n=0,1,2,3}$ is a valid contravariant pseudo-metric. Moreover, the generalized-Lorentz's structure, given by $\left\{K^{m n}\right\}_{m, n=0,1,2,3}$ is strongly correlated with the global time $\varphi$. Finally, we also have

$$
v^{m}=\sum_{j=0}^{3} K^{j m} \frac{\partial \varphi}{\partial x^{j}} \quad \forall m=0,1,2,3 \quad \forall\left(x^{0}, x^{1}, x^{2}, x^{3}\right) \in \mathbb{R}^{4},
$$

So, $\left\{\Lambda^{m n}\right\}_{m, n=0,1,2,3}$ is a contravariant tensor of three-dimensional Geometry and $\left(v^{0}, v^{1}, v^{2}, v^{3}\right)$ is a potential of generalized gravity, corresponding to the pseudo-metric $K^{m n}$ and the global time $\varphi$.

Remark 2.1. The above Proposition and Corollary show that the Galilean structure alone incompletely describes the geometry of the space-time and for complete description, in addition to $\left\{\Lambda^{m n}\right\}_{m, n=0,1,2,3}$ and $\left(w_{0}, w_{1}, w_{2}, w_{3}\right)$, above we need to specify one more contravariant vector field $\left(v^{0}, v^{1}, v^{2}, v^{3}\right)$ (that we call here the potential of generalized-gravity) satisfying either

$$
\sum_{j=0}^{3} v^{j} w_{j}>0 \quad \forall\left(x^{0}, x^{1}, x^{2}, x^{3}\right) \in \mathbb{R}^{4},
$$

in the case of the weak coupling, or

$$
\sum_{j=0}^{3} v^{j} w_{j}=1 \quad \forall\left(x^{0}, x^{1}, x^{2}, x^{3}\right) \in \mathbb{R}^{4},
$$

in the case of the strong coupling. Then we can define a Lorentz's-like contravariant pseudo-metric $\left\{K^{m n}\right\}_{m, n=0,1,2,3}$ as:

$$
K^{m n}=v^{j} v^{m}-\Lambda^{j m} \quad \forall j, m=0,1,2,3,
$$

that will either weakly or strongly correlate with the time direction $\left(w_{0}, w_{1}, w_{2}, w_{3}\right)$. Moreover, in the case of strong coupling, the contravariant vector $\left(v^{0}, v^{1}, v^{2}, v^{3}\right)$ is just a lifted time-direction $\left(w_{0}, w_{1}, w_{2}, w_{3}\right)$ with respect to a pseudo-metric $\left\{K^{m n}\right\}_{m, n=0,1,2,3}$. Very similar to $\left(v^{0}, v^{1}, v^{2}, v^{3}\right)$ contravariant vector field appears implicitly in in the equation (2.2) and before it on [5] (it was denoted $\left(\nu^{0}, \nu^{1}, \nu^{2}, \nu^{3}\right)$ there). Moreover, then it can be easily shown that the covariant degenerate tensor field $\left\{h_{m n}\right\}_{m, n=0,1,2,3}$ in (2.2) on [5] is just the lowering-index of the tensor $\left\{h^{m n}\right\}_{m, n=0,1,2,3}:=$ $\left\{\Lambda^{m n}\right\}_{m, n=0,1,2,3}$, with respect to the covariant pseudo-metric $\left\{K_{m n}\right\}_{m, n=0,1,2,3}$, which is inverse to the contravariant pseudo-metric

$$
K^{m n}=v^{j} v^{m}-\Lambda^{j m}:=\nu^{j} \nu^{m}-h^{j m} \quad \forall j, m=0,1,2,3 .
$$

Definition 2.5. Consider a contravariant pseudo-metric $\left\{K^{m n}\right\}_{m, n=0,1,2,3}$ on $\mathbb{R}^{4}$ and let $\left\{K_{m n}\right\}_{m, n=0,1,2,3}$ be the inverse covariant pseudo-metric on $\mathbb{R}^{4}$, associated with $\left\{K^{m n}\right\}_{m, n=0,1,2,3}$. Then, in every coordinate system define the Christoffel Symbols $\left\{\Gamma_{k j}^{m}\right\}_{K}$ by

$$
\left\{\begin{array}{ll}
\left\{\Gamma_{j, m n}\right\}_{K}:=\frac{1}{2}\left(\frac{\partial K_{j m}}{\partial x^{n}}+\frac{\partial K_{j n}}{\partial x^{m}}-\frac{\partial K_{m n}}{\partial x^{j}}\right) & \forall j, m, n=0,1,2,3 . \\
\left\{\Gamma_{m n}^{j}\right\}_{K}:=\sum_{k=0}^{3} K^{j k}\left\{\Gamma_{k, m n}\right\}_{K} &
\end{array} \quad \forall j\right.
$$


Furthermore, given a covariant four-vector field $\left(h_{0}, h_{1}, h_{2}, h_{3}\right)$, define the covariant derivative of $\left(h_{0}, h_{1}, h_{2}, h_{3}\right)$ by

$$
\left\{\delta_{j} h_{k}\right\}_{K}:=\frac{\partial h_{k}}{\partial x^{j}}-\sum_{m=0}^{3}\left\{\Gamma_{k j}^{m}\right\}_{K} h_{m} \quad \forall k, j=0,1,2,3 .
$$

Then, it is well known that $\left\{\left\{\delta_{n} h_{m}\right\}_{K}\right\}_{m, n=0,1,2,3}$ is a two-times covariant tensor.

Definition 2.6. Consider a contravariant pseudo-metric $\left\{K^{m n}\right\}_{m, n=0,1,2,3}$ on $\mathbb{R}^{4}$ and let $\left\{K_{m n}\right\}_{m, n=0,1,2,3}$ be the inverse covariant pseudo-metric on $\mathbb{R}^{4}$, associated with $\left\{K^{m n}\right\}_{m, n=0,1,2,3}$. Next, consider the Christoffel Symbols $\left\{\Gamma_{k j}^{m}\right\}_{K}$, defined by (2.25). Given a coordinate system in $\mathbb{R}^{4}$, we call it inertial coordinate system with respect to $\left\{K^{m n}\right\}_{m, n=0,1,2,3}$ if, in this particular coordinate system we have

$$
\left\{\Gamma_{m n}^{j}\right\}_{K}=0 \quad \forall j, m, n=0,1,2,3 .
$$

Then, using Lemma 11.2 from the Appendix, we deduce that, given coordinate system in $\mathbb{R}^{4}$ is inertial with respect to $\left\{K^{m n}\right\}_{m, n=0,1,2,3}$ if and only if the tensor $\left\{K_{m n}\right\}_{m, n=0,1,2,3}$ is independent on the local coordinates $\left(x^{0}, x^{1}, x^{2}, x^{3}\right) \in \mathbb{R}^{4}$ in this particular coordinate system.

Definition 2.7. If there exists some coordinate system where matrix $\left\{K^{m n}\right\}_{m, n=0,1,2,3}$ has a form of

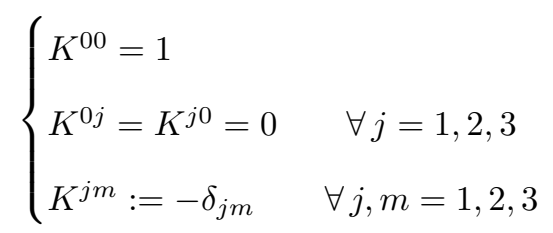

at every point in $\mathbb{R}^{4}$ and at the same time the covector of time direction for $\varphi$ has a form

$$
\left(\frac{\partial \varphi}{\partial x^{0}}, \frac{\partial \varphi}{\partial x^{1}}, \frac{\partial \varphi}{\partial x^{2}}, \frac{\partial \varphi}{\partial x^{3}}\right)\left(x^{0}, x^{1}, x^{2}, x^{3}\right)=(1,0,0,0) \quad \forall\left(x^{0}, x^{1}, x^{2}, x^{3}\right) \in \mathbb{R}^{4}
$$

in the same coordinate system, then the contravariant pseudo-metric $\left\{K^{m n}\right\}_{m, n=0,1,2,3}$ and the global time $\varphi$ are obviously strongly correlated on $\mathbb{R}^{4}$ and we say that the standard kinematical Lorentz's structure with global time on $\mathbb{R}^{4}$ is chosen. Moreover, in that case we call the contrvariant four-vector field of the potential of generalized gravity $\left(v^{0}, v^{1}, v^{2}, v^{3}\right)$, corresponding to that structure and defined, as in (2.7), by

$$
v^{m}:=\sum_{j=0}^{3} K^{m j} \frac{\partial \varphi}{\partial x^{j}} \quad \forall m=0,1,2,3,
$$

the contrvariant four-vector of the potential of inertia. Furthermore, we call the coordinate system where (2.28) and (2.29) hold simultaneously, kinematically-preferable for the given generalizedLorentz's structure with the global time. In particular, it is clear that a kinematically-preferable system is inertial with respect to $\left\{K^{m n}\right\}_{m, n=0,1,2,3}$ (see Definition 2.6) Furthermore, in kinematicallypreferable coordinate system we obviously have

$$
\left(v^{0}, v^{1}, v^{2}, v^{3}\right)\left(x^{0}, x^{1}, x^{2}, x^{3}\right)=(1,0,0,0) \quad \forall\left(x^{0}, x^{1}, x^{2}, x^{3}\right) \in \mathbb{R}^{4} .
$$


Moreover, in the same coordinate system the contravariant tensor field $\left\{\Lambda^{m n}\right\}_{m, n=0,1,2,3}$, given as in (2.11) by

$$
\Lambda^{j m}:=v^{j} v^{m}-K^{j m} \quad \forall j, m=0,1,2,3,
$$

obviously satisfies

$$
\left\{\begin{array}{l}
\Lambda^{00}=0 \\
\Lambda^{0 j}=\Lambda^{j 0}=0 \quad \forall j=1,2,3 \\
\Lambda^{j m}:=\delta_{j m} \quad \forall j, m=1,2,3 .
\end{array}\right.
$$

Finally, using Lemma 11.3 from the Appendix in the end, we deduce that a general coordinate system is inertial, with respect to the above pseudo-metric, if this coordinate system is obtained from kinematically-preferable system by a general linear transformation of the form:

$$
x^{\prime m}=\sum_{j=0}^{3} C_{m j} x^{j}+c^{m} \quad \forall m=0,1,2,3,
$$

where $\left\{C_{m n}\right\}_{n, m=1,2,3} \in \mathbb{R}^{4 \times 4}$ is a constant (independent on $\left.\left(x^{0}, x^{1}, x^{2}, x^{3}\right) \in \mathbb{R}^{4}\right)$ non-degenerate matrix, and $\left(c^{0}, c^{1}, c^{2}, c^{3}\right) \in \mathbb{R}^{4}$ is a constant (independent on $\left.\left(x^{0}, x^{1}, x^{2}, x^{3}\right) \in \mathbb{R}^{4}\right)$ vector.

Definition 2.8. Given a contravariant pseudo-metric $\left\{K^{m n}\right\}_{m, n=0,1,2,3}$, we say that a given general coordinate system in $\mathbb{R}^{4}$ is Lorentzian with respect to $\left\{K^{m n}\right\}_{m, n=0,1,2,3}$, if $\left\{K^{m n}\right\}_{m, n=0,1,2,3}$ has the following simple form in the chosen coordinate system:

$$
\begin{cases}K^{00}=1 \quad \forall\left(x^{0}, x^{1}, x^{2}, x^{3}\right) \in \mathbb{R}^{4} & \\ K^{m n}=-\delta_{m n} \quad \forall 1 \leq m, n \leq 3 & \forall\left(x^{0}, x^{1}, x^{2}, x^{3}\right) \in \mathbb{R}^{4} \\ K^{0 j}=K^{j 0}=0 \quad \forall 1 \leq j \leq 3 & \forall\left(x^{0}, x^{1}, x^{2}, x^{3}\right) \in \mathbb{R}^{4} .\end{cases}
$$

Note that (2.35) also implies:

$$
\left\{\begin{array}{l}
K_{00}=1 \\
K_{m n}=-\delta_{m n} \quad \forall 1 \leq m, n \leq 3 \\
K_{0 j}=K_{j 0}=0 \quad \forall 1 \leq j \leq 3
\end{array}\right.
$$

Moreover, due to Definition 2.6, every Lorentzian coordinate system is obviously inertial with respect to $\left\{K^{m n}\right\}_{m, n=0,1,2,3}$.

Definition 2.9. Let

$$
\left\{\begin{array}{l}
x^{\prime 0}=f^{(0)}\left(x^{0}, x^{1}, x^{2}, x^{3}\right), \\
x^{\prime 1}=f^{(1)}\left(x^{0}, x^{1}, x^{2}, x^{3}\right), \\
x^{\prime 2}=f^{(2)}\left(x^{0}, x^{1}, x^{2}, x^{3}\right), \\
x^{\prime 3}=f^{(3)}\left(x^{0}, x^{1}, x^{2}, x^{3}\right) .
\end{array}\right.
$$


be a change of the first general coordinate system to the second coordinate system. We say that transformations (2.37) are Lorentz's transformations if, for arbitrary contravariant pseudo-metric $\left\{K^{m n}\right\}_{m, n=0,1,2,3}$, such that in the first coordinate system we have

$$
\left\{\begin{array}{l}
K^{00}=1 \quad \forall\left(x^{0}, x^{1}, x^{2}, x^{3}\right) \in \mathbb{R}^{4} \\
K^{m n}=-\delta_{m n} \quad \forall 1 \leq m, n \leq 3 \quad \forall\left(x^{0}, x^{1}, x^{2}, x^{3}\right) \in \mathbb{R}^{4} \\
K^{0 j}=K^{j 0}=0 \quad \forall 1 \leq j \leq 3 \quad \forall\left(x^{0}, x^{1}, x^{2}, x^{3}\right) \in \mathbb{R}^{4},
\end{array}\right.
$$

in the second coordinate system we also have

$$
\left\{\begin{array}{l}
K^{\prime 00}=1 \quad \forall\left(x^{\prime 0}, x^{\prime 1}, x^{\prime 2}, x^{\prime 3}\right) \in \mathbb{R}^{4} \\
K^{\prime m n}=-\delta_{m n} \quad \forall 1 \leq m, n \leq 3 \quad \forall\left(x^{\prime 0}, x^{\prime 1}, x^{\prime 2}, x^{\prime 3}\right) \in \mathbb{R}^{4} \\
K^{\prime 0 j}=K^{\prime j 0}=0 \quad \forall 1 \leq j \leq 3 \quad \forall\left(x^{\prime 0}, x^{\prime 1}, x^{\prime 2}, x^{\prime 3}\right) \in \mathbb{R}^{4} .
\end{array}\right.
$$

In particular, observe that, using Lemma 11.3 from the Appendix, we deduce that every Lorentz's transformation is necessarily linear.

Definition 2.10. Consider a standard kinematical Lorentz's structure with global time $\varphi$ on $\mathbb{R}^{4}$, together with the corresponding tensor of three-dimensional Geometry $\left\{\Lambda^{m n}\right\}_{m, n=0,1,2,3}$. We say that a given general coordinate system in $\mathbb{R}^{4}$ is cartesian with respect to the tensor of the threedimensional geometry $\left\{\Lambda^{m n}\right\}_{m, n=0,1,2,3}$ and the global time $\varphi$, if $\left\{\Lambda^{m n}\right\}_{m, n=0,1,2,3}$ has the following simple form in the chosen coordinate system:

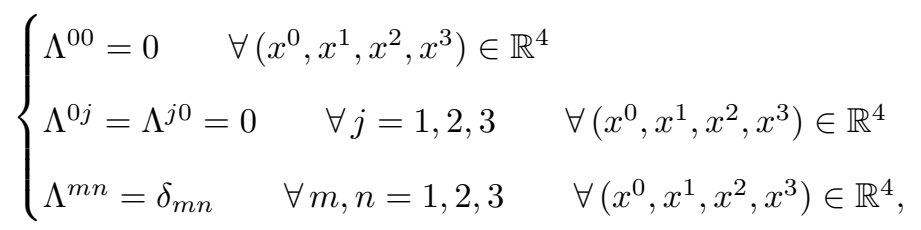

and at the same time in the same coordinate system we have

$$
\left(\frac{\partial \varphi}{\partial x^{0}}, \frac{\partial \varphi}{\partial x^{1}}, \frac{\partial \varphi}{\partial x^{2}}, \frac{\partial \varphi}{\partial x^{3}}\right)\left(x^{0}, x^{1}, x^{2}, x^{3}\right)=(1,0,0,0) \quad \forall\left(x^{0}, x^{1}, x^{2}, x^{3}\right) \in \mathbb{R}^{4} .
$$

Definition 2.11. We say that two cartesian coordinate systems in $\mathbb{R}^{4}$ are equivalent if the change of coordinates from one system to another is given by

$$
\left\{\begin{array}{l}
x^{\prime 0}=x^{0}+c^{0}, \\
x^{\prime m}=\sum_{j=1}^{3} B_{m j} x^{j}+c^{m} \quad \forall m=1,2,3,
\end{array}\right.
$$

where $\left\{B_{m n}\right\}_{n, m=1,2,3} \in \mathbb{R}^{3 \times 3}$ is a constant (independent on $\left(x^{0}, x^{1}, x^{2}, x^{3}\right) \in \mathbb{R}^{4}$ ) matrix, satisfying

$$
\sum_{j=1}^{3} B_{m j} B_{n j}=\sum_{j=1}^{3} B_{j m} B_{j n}=\delta_{m n} \quad \forall m, n=1,2,3,
$$

and $\left(c^{0}, c^{1}, c^{2}, c^{3}\right) \in \mathbb{R}^{4}$ is a constant (independent on $\left.\left(x^{0}, x^{1}, x^{2}, x^{3}\right) \in \mathbb{R}^{4}\right)$ vector. 
Then we prove the following:

Theorem 2.1. Consider a standard kinematical Lorentz's structure with global time on $\mathbb{R}^{4}$ together with the corresponding tensor of three-dimensional Geometry $\left\{\Lambda^{m n}\right\}_{m, n=0,1,2,3}$. Next consider the first cartesian, with respect to $\left\{\Lambda^{m n}\right\}_{m, n=0,1,2,3}$ and $\varphi$, coordinate system in $\mathbb{R}^{4}$ and the second general coordinate system in $\mathbb{R}^{4}$. Then, the second coordinate system is also cartesian if and only if the change of the first coordinate system to the second one is given by the following relations:

$$
\left\{\begin{array}{l}
x^{\prime 0}=x^{0}+c \\
x^{\prime m}=\sum_{j=1}^{3} A_{m j}\left(x^{0}\right) x^{j}+z^{m}\left(x^{0}\right) \quad \forall m=1,2,3,
\end{array}\right.
$$

where $\left\{A_{m n}\left(x^{0}\right)\right\}_{n, m=1,2,3} \in \mathbb{R}^{3 \times 3}$ is a $3 \times 3$-matrix, depending on the coordinate $x^{0}$ only (independent on $\left.\mathbf{x}:=\left(x^{1}, x^{2}, x^{3}\right)\right)$, and satisfying

$$
\sum_{j=1}^{3} A_{m j}\left(x^{0}\right) A_{n j}\left(x^{0}\right)=\sum_{j=1}^{3} A_{j m}\left(x^{0}\right) A_{j n}\left(x^{0}\right)=\delta_{m n} \quad \forall m, n=1,2,3 \quad \forall\left(x^{0}, x^{1}, x^{2}, x^{3}\right) \in \mathbb{R}^{4},
$$

$c \in \mathbb{R}$ is a constant (independent on $\left.\left(x^{0}, x^{1}, x^{2}, x^{3}\right) \in \mathbb{R}^{4}\right)$ and $\mathbf{z}\left(x^{0}\right):=\left(z^{1}\left(x^{0}\right), z^{2}\left(x^{0}\right), z^{3}\left(x^{0}\right)\right) \in$ $\mathbb{R}^{3}$ is a three-dimensional vector field, depending on the coordinate $x^{0}$ only (independent on $\mathbf{x}:=$ $\left.\left(x^{1}, x^{2}, x^{3}\right)\right)$. In particular, up to equivalence of cartesian coordinate systems (see Definition 2.11), (2.44) reduces to

$$
\left\{\begin{array}{l}
x^{\prime 0}=x^{0} \\
\mathbf{x}^{\prime}=A\left(x^{0}\right) \cdot \mathbf{x}+\mathbf{z}\left(x^{0}\right),
\end{array}\right.
$$

where $A\left(x^{0}\right) \in S O(3)$ is a rotation, depending on the coordinate $x^{0}$ only and where $\mathbf{z}\left(x^{0}\right)$ is a three-dimensional vector field, depending on the coordinate $x^{0}$ only.

As a consequence of Theorem 2.1 together with Lemma 11.3 from the Appendix, we deduce the following:

Corollary 2.3. Consider a standard kinematical Lorentz's structure with global time on $\mathbb{R}^{4}$. Next consider the first coordinate system in $\mathbb{R}^{4}$, which is simultaneously inertial and cartesian, with respect to this structure, and the second general coordinate system in $\mathbb{R}^{4}$. Then, the second coordinate system is also simultaneously inertial and cartesian if and only if the change of the first coordinate system to the second one is given by the following relations:

$$
\left\{\begin{array}{l}
x^{\prime 0}=x^{0}+c^{0}, \\
x^{\prime m}=\sum_{j=1}^{3} B_{m j} x^{j}+w^{m} x^{0}+c^{m} \quad \forall m=1,2,3,
\end{array}\right.
$$

where $\left\{B_{m n}\right\}_{n, m=1,2,3} \in \mathbb{R}^{3 \times 3}$ is a constant $3 \times 3$-matrix (independent on $\left(x^{0}, x^{1}, x^{2}, x^{3}\right) \in \mathbb{R}^{4}$ ), and satisfying

$$
\sum_{j=1}^{3} B_{m j} B_{n j}=\sum_{j=1}^{3} B_{j m} B_{j n}=\delta_{m n} \quad \forall m, n=1,2,3
$$


$\left(c^{0}, c^{1}, c^{2}, c^{3}\right) \in \mathbb{R}^{4}$ is a constant vector (independent on $\left.\left(x^{0}, x^{1}, x^{2}, x^{3}\right) \in \mathbb{R}^{4}\right)$ and $\mathbf{w}:=\left(w^{1}, w^{2}, w^{3}\right) \in$ $\mathbb{R}^{3}$ is a constant three-dimensional vector (independent on $\left(x^{0}, x^{1}, x^{2}, x^{3}\right) \in \mathbb{R}^{4}$ ). In particular, up to equivalence of cartesian coordinate systems (see Definition 2.11), (2.47) reduces to the classical Galilean Transformations:

$$
\left\{\begin{array}{l}
x^{\prime 0}=x^{0} \\
x^{\prime m}=x^{m}+w^{m} x^{0} \quad \forall m=1,2,3,
\end{array}\right.
$$

where $\mathbf{w}:=\left(w^{1}, w^{2}, w^{3}\right) \in \mathbb{R}^{3}$ is a constant three-dimensional vector field (independent on the point $\left.\left(x^{0}, x^{1}, x^{2}, x^{3}\right) \in \mathbb{R}^{4}\right)$.

Theorem 2.2. Consider a standard kinematical Lorentz's structure with global time on $\mathbb{R}^{4}$. Next consider the first coordinate system in $\mathbb{R}^{4}$, which is kinematically-preferable and the second general coordinate system in $\mathbb{R}^{4}$. Then, the second coordinate system is also kinematically-preferable, if and only if the first and the second coordinate systems are equivalent cartesian systems.

Remark 2.2. Consider a standard kinematical Lorentz's structure with global time on $\mathbb{R}^{4}$. Next consider a coordinate system in $\mathbb{R}^{4}$, which is simultaneously cartesian and Lorentzian, with respect to this structure. Then, by Definition 2.7 this system is kinematically-preferable, with respect to this structure. Moreover, note that, all kinematically-preferable systems are equivalent cartesian and Lorentzian systems. Finally, note that the arbitrary coordinate system is Lorentzian if and only if it can be obtained from the kinematically-preferable system by some Lorentz's transformation.

\subsubsection{Pseudo-Lorentzian coordinate systems}

Definition 2.12. Consider a standard kinematical Lorentz's structure with global time on $\mathbb{R}^{4}$ (see Definition 2.7), consisting of contravariant pseudo-metric $\left\{K^{m n}\right\}_{m, n=0,1,2,3}$ and the global time $\varphi$, together with the corresponding tensor of three-dimensional Geometry $\left\{\Lambda^{m n}\right\}_{m, n=0,1,2,3}$, defined, as in $(2.32)$ by

$$
\Lambda^{j m}:=v^{j} v^{m}-K^{j m} \quad \forall j, m=0,1,2,3,
$$

where $\left(v^{0}, v^{1}, v^{2}, v^{3}\right)$ is the contrvariant four-vector field of the potential of generalized gravity, given, as in $(2.30)$, by

$$
v^{m}:=\sum_{j=0}^{3} K^{m j} \frac{\partial \varphi}{\partial x^{j}} \quad \forall m=0,1,2,3 .
$$

We say that a given general coordinate system in $\mathbb{R}^{4}$ is Pseudo-Lorentzian with respect to the tensor of the three-dimensional geometry $\left\{\Lambda^{m n}\right\}_{m, n=0,1,2,3}$ and the global time $\varphi$, if $\left\{\Lambda^{m n}\right\}_{m, n=0,1,2,3}$ has the following simple form in the chosen coordinate system:

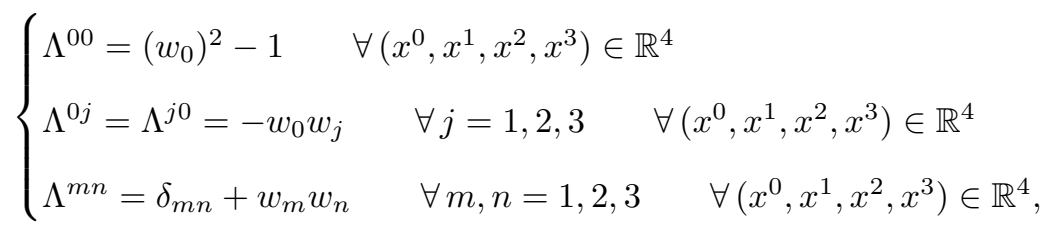


and at the same time in the same coordinate system we have

$$
\left(\frac{\partial \varphi}{\partial x^{0}}, \frac{\partial \varphi}{\partial x^{1}}, \frac{\partial \varphi}{\partial x^{2}}, \frac{\partial \varphi}{\partial x^{3}}\right)\left(x^{0}, x^{1}, x^{2}, x^{3}\right)=\left(w_{0}, w_{1}, w_{2}, w_{3}\right) \quad \forall\left(x^{0}, x^{1}, x^{2}, x^{3}\right) \in \mathbb{R}^{4},
$$

where $\left(w_{0}, w_{1}, w_{2}, w_{3}\right) \in \mathbb{R}^{4}$ is some constant (independent on the point $\left.\left(x^{0}, x^{1}, x^{2}, x^{3}\right) \in \mathbb{R}^{4}\right)$ vector, satisfying

$$
\left(w_{0}\right)^{2}-\sum_{j=1}^{3}\left(w_{j}\right)^{2}=1,
$$

(Note that by Lemma 11.4 the matrix $\left\{\Lambda^{m n}\right\}_{0 \leq m, n \leq 3}$ in (2.52) is degenerate and moreover, it has one vanishing and three positive eigenvalues).

Remark 2.3. Obviously every Lorentzian coordinate system, where

$$
\begin{cases}K^{00}=1 & \\ K^{0 j}=K^{j 0}=0 & \forall j=1,2,3 \\ K^{m n}:=-\delta_{m n} & \forall m, n=1,2,3,\end{cases}
$$

is a Pseudo-Lorentzian system, since such a system obtained from the kinematically-preferable system by a linear Lorentz's transformation. Moreover, every cartesian coordinate system is also Pseudo-Lorentzian system with

$$
\left(\frac{\partial \varphi}{\partial x^{0}}, \frac{\partial \varphi}{\partial x^{1}}, \frac{\partial \varphi}{\partial x^{2}}, \frac{\partial \varphi}{\partial x^{3}}\right)\left(x^{0}, x^{1}, x^{2}, x^{3}\right)=(1,0,0,0) \quad \forall\left(x^{0}, x^{1}, x^{2}, x^{3}\right) \in \mathbb{R}^{4} .
$$

Theorem 2.3. Given coordinate system is Pseudo-Lorentzian, if and only if it obtained from some cartesian coordinate system by some Lorentz's transformation.

Definition 2.13. In every coordinate system (possibly curvilinear) we consider the following matrices

$$
\left\{\begin{array}{l}
M^{00}:=1 \\
M^{0 j}=M^{j 0}:=0 \quad \forall j=1,2,3 \\
M^{j m}:=-\delta_{j m} \quad \forall j, m=1,2,3,
\end{array}\right.
$$

and

$$
\left\{\begin{array}{l}
M_{00}:=1 \\
M_{0 j}=M_{j 0}:=0 \quad \forall j=1,2,3 \\
M_{j m}:=-\delta_{j m} \quad \forall j, m=1,2,3,
\end{array}\right.
$$

(note that $M^{k j}$ and $M_{k j}$ are not tensors). Next for given fixed constant vector $\left(w_{0}, w_{1}, w_{2}, w_{3}\right) \in \mathbb{R}^{4}$, satisfying

$$
\sum_{j=0}^{3} \sum_{m=0}^{3} M^{j m} w_{j} w_{m}=1
$$


we say that the change of coordinate system $\left(x^{0}, x^{1}, x^{2}, x^{3}\right) \rightarrow\left(x^{\prime 0}, x^{\prime 1}, x^{\prime 2}, x^{\prime 3}\right)$ is of the class $P L\left(w_{0}, w_{1}, w_{2}, w_{3}\right)$ if we have

$$
x^{\prime m}:=\sum_{j=0}^{3} B_{m j}\left(\sum_{k=0}^{3} w_{k} x^{k}\right) x^{j}+z^{m}\left(\sum_{k=0}^{3} w_{k} x^{k}\right) \quad \forall m=0,1,2,3,
$$

where $B(s):=\left\{B_{m j}(s)\right\}_{m, j=0,1,2,3}: \mathbb{R} \rightarrow \mathbb{R}^{4 \times 4}$ and $\left(z^{0}(s), z^{1}(s), z^{2}(s), z^{3}(s)\right): \mathbb{R} \rightarrow \mathbb{R}^{4}$ satisfy

$$
\begin{gathered}
M^{m n}=\sum_{j=0}^{3} \sum_{k=0}^{3} B_{m j}(s) B_{n k}(s) M^{j k} \quad \forall m, n=0,1,2,3, \quad \forall s \in \mathbb{R}, \\
\sum_{j=0}^{3} w_{j}\left(\left\{B^{-1} \cdot \frac{d B}{d s}\right\}_{j m}(s)\right)=0 \quad \forall m=0,1,2,3, \quad \forall s \in \mathbb{R},
\end{gathered}
$$

and

$$
\sum_{m=0}^{3} \sum_{j=0}^{3} w_{j}\left(\left\{B^{-1}\right\}_{j m}(s)\right) \frac{d z^{m}}{d s}(s)=0 \quad \forall s \in \mathbb{R} .
$$

As a direct consequence of Definition 2.13 we have the following:

Corollary 2.4. Given change of coordinate system $\left(x^{0}, x^{1}, x^{2}, x^{3}\right) \rightarrow\left(x^{\prime 0}, x^{\prime 1}, x^{\prime 2}, x^{\prime 3}\right)$ is a Lorentz's transformation, if and only if this transformation is of class $P L\left(w_{0}, w_{1}, w_{2}, w_{3}\right)$ for every fixed constant vector $\left(w_{0}, w_{1}, w_{2}, w_{3}\right) \in \mathbb{R}^{4}$ satisfying (2.57), where $B:=\left\{B_{m j}\right\}_{m, j=0,1,2,3} \in \mathbb{R}^{4 \times 4}$ and $\left(z^{0}, z^{1}, z^{2}, z^{3}\right) \in \mathbb{R}^{4}$ in (2.58) are independent on the argument $s$.

As the second direct consequence of Definition 2.13 and Theorem 2.1 we have the following:

Corollary 2.5. Consider a standard kinematical Lorentz's structure with global time $\varphi$ on $\mathbb{R}^{4}$ together with the corresponding tensor of three-dimensional Geometry $\left\{\Lambda^{m n}\right\}_{m, n=0,1,2,3}$. Next consider the first cartesian, with respect to $\left\{\Lambda^{m n}\right\}_{m, n=0,1,2,3}$ and $\varphi$, coordinate system in $\mathbb{R}^{4}$ and the second general coordinate system in $\mathbb{R}^{4}$. Then, the second coordinate system is also cartesian if and only if the change of the first coordinate system to the second one is of class $P L(1,0,0,0)$ where $B(s):=\left\{B_{m j}(s)\right\}_{m, j=0,1,2,3}: \mathbb{R} \rightarrow \mathbb{R}^{4 \times 4}$ in $(2.58)$ is given by

$$
\left\{\begin{array}{l}
B_{00}(s)=1 \quad \forall s \\
B_{0 j}(s)=B_{j 0}(s)=0 \quad \forall j=1,2,3 \quad \forall s \\
B_{m n}(s)=A_{m n}(s) \quad \forall m, n=1,2,3 \quad \forall s,
\end{array}\right.
$$

where $\left\{A_{m n}(s)\right\}_{n, m=1,2,3} \in \mathbb{R}^{3 \times 3}$ is a $3 \times 3$-matrix, satisfying

$$
\sum_{j=1}^{3} A_{m j}(s) A_{n j}(s)=\sum_{j=1}^{3} A_{j m}(s) A_{j n}(s)=\delta_{m n} \quad \forall m, n=1,2,3 \quad \forall s .
$$

Theorem 2.4. Consider a fixed Pseudo-Lorentzian coordinate system, so that

$$
\begin{cases}\Lambda^{00}=\left(w_{0}\right)^{2}-1 & \forall\left(x^{0}, x^{1}, x^{2}, x^{3}\right) \in \mathbb{R}^{4} \\ \Lambda^{0 j}=\Lambda^{j 0}=-w_{0} w_{j} & \forall j=1,2,3 \quad \forall\left(x^{0}, x^{1}, x^{2}, x^{3}\right) \in \mathbb{R}^{4} \\ \Lambda^{m n}=\delta_{m n}+w_{m} w_{n} & \forall m, n=1,2,3 \quad \forall\left(x^{0}, x^{1}, x^{2}, x^{3}\right) \in \mathbb{R}^{4},\end{cases}
$$


and at the same time in the same coordinate system we have

$$
\left(\frac{\partial \varphi}{\partial x^{0}}, \frac{\partial \varphi}{\partial x^{1}}, \frac{\partial \varphi}{\partial x^{2}}, \frac{\partial \varphi}{\partial x^{3}}\right)\left(x^{0}, x^{1}, x^{2}, x^{3}\right)=\left(w_{0}, w_{1}, w_{2}, w_{3}\right) \quad \forall\left(x^{0}, x^{1}, x^{2}, x^{3}\right) \in \mathbb{R}^{4}
$$

where $\left(w_{0}, w_{1}, w_{2}, w_{3}\right) \in \mathbb{R}^{4}$ is some constant (independent on the point $\left(x^{0}, x^{1}, x^{2}, x^{3}\right) \in \mathbb{R}^{4}$ ) vector, satisfying

$$
\left(w_{0}\right)^{2}-\sum_{j=1}^{3}\left(w_{j}\right)^{2}=1
$$

Next, assume that the new coordinate system is obtained from the given above (old) system by the following transformation

$$
\left(x^{0}, x^{1}, x^{2}, x^{3}\right) \rightarrow\left(x^{\prime \prime \prime}, x^{\prime \prime \prime 1}, x^{\prime \prime \prime 2}, x^{\prime \prime \prime 3}\right)
$$

Then the new coordinate system is also Pseudo-Lorentzian if and only if the transformation in (2.67) is of class $P L\left(\left(w_{0}, w_{1}, w_{2}, w_{3}\right)\right)$.

Proposition 2.3. Given a constant vector $\left(w_{0}, w_{1}, w_{2}, w_{3}\right) \in \mathbb{R}^{4}$, satisfying $(2.57)$ assume that that the change of coordinate system $\left(x^{0}, x^{1}, x^{2}, x^{3}\right) \rightarrow\left(x^{\prime 0}, x^{\prime 1}, x^{\prime 2}, x^{\prime 3}\right)$ is of class $P L\left(w_{0}, w_{1}, w_{2}, w_{3}\right)$ and consider another constant vector $\left(w_{0}^{\prime}, w_{1}^{\prime}, w_{2}^{\prime}, w_{3}^{\prime}\right) \in \mathbb{R}^{4}$, defined by

$$
w_{m}^{\prime}=\sum_{j=0}^{3} w_{j}\left(\left\{B^{-1}\right\}_{j m}(0)\right) \quad \forall m=0,1,2,3, \quad \forall s \in \mathbb{R},
$$

where $B(s):=\left\{B_{m j}(s)\right\}_{m, j=0,1,2,3}: \mathbb{R} \rightarrow \mathbb{R}^{4 \times 4}$ be as in (2.58). Then, we have

$$
\sum_{m=0}^{3} \sum_{n=0}^{3} M^{m n} w_{m}^{\prime} w_{n}^{\prime}=1
$$

$$
\sum_{j=0}^{3} w_{j}\left(\left\{B^{-1}\right\}_{j m}(s)\right)=\sum_{j=0}^{3} w_{j}\left(\left\{B^{-1}\right\}_{j m}(0)\right)=w_{m}^{\prime} \quad \forall m=0,1,2,3, \quad \forall s \in \mathbb{R} .
$$

Moreover, at every point in $\mathbb{R}^{4}$ we have

$$
\sum_{m=0}^{3} w_{m}^{\prime} \frac{\partial x^{\prime m}}{\partial x^{n}}=w_{n} \quad \forall n=0,1,2,3,
$$

and as a direct consequence of (2.71) there exists a constant $C \in \mathbb{R}$ such that at every point in $\mathbb{R}^{4}$ we have

$$
\left(\sum_{k=0}^{3} w_{k}^{\prime} x^{\prime k}\right)=C+\left(\sum_{k=0}^{3} w_{k} x^{k}\right) .
$$

Definition 2.14. Given two fixed constant vectors $\left(w_{0}, w_{1}, w_{2}, w_{3}\right) \in \mathbb{R}^{4}$ and $\left(w_{0}^{\prime}, w_{1}^{\prime}, w_{2}^{\prime}, w_{3}^{\prime}\right) \in \mathbb{R}^{4}$, satisfying

$$
\sum_{j=0}^{3} \sum_{m=0}^{3} M^{j m} w_{j} w_{m}=1
$$

and

$$
\sum_{j=0}^{3} \sum_{m=0}^{3} M^{j m} w_{j}^{\prime} w_{m}^{\prime}=1
$$


we say that the change of coordinate system $\left(x^{0}, x^{1}, x^{2}, x^{3}\right) \rightarrow\left(x^{\prime 0}, x^{\prime 1}, x^{\prime 2}, x^{\prime 3}\right)$ is of the class $P L\left(\left(w_{0}, w_{1}, w_{2}, w_{3}\right) ;\left(w_{0}^{\prime}, w_{1}^{\prime}, w_{2}^{\prime}, w_{3}^{\prime}\right)\right)$ if it belongs to $P L\left(w_{0}, w_{1}, w_{2}, w_{3}\right)$ and

$$
w_{m}^{\prime}=\sum_{j=0}^{3} w_{j}\left(\left\{B^{-1}\right\}_{j m}(0)\right) \quad \forall m=0,1,2,3, \quad \forall s \in \mathbb{R},
$$

where $B(s):=\left\{B_{m j}(s)\right\}_{m, j=0,1,2,3}: \mathbb{R} \rightarrow \mathbb{R}^{4 \times 4}$ be as in (2.58).

Furthermore, for given two fixed constant vectors $\left(w_{0}, w_{1}, w_{2}, w_{3}\right) \in \mathbb{R}^{4}$ and $\left(w_{0}^{\prime}, w_{1}^{\prime}, w_{2}^{\prime}, w_{3}^{\prime}\right) \in \mathbb{R}^{4}$, satisfying (2.73) and (2.74) we define the subspace

$$
L\left(\left(w_{0}, w_{1}, w_{2}, w_{3}\right) ;\left(w_{0}^{\prime}, w_{1}^{\prime}, w_{2}^{\prime}, w_{3}^{\prime}\right)\right) \subsetneq P L\left(\left(w_{0}, w_{1}, w_{2}, w_{3}\right) ;\left(w_{0}^{\prime}, w_{1}^{\prime}, w_{2}^{\prime}, w_{3}^{\prime}\right)\right)
$$

of transformations $\left(x^{0}, x^{1}, x^{2}, x^{3}\right) \rightarrow\left(x^{\prime 0}, x^{\prime 1}, x^{\prime 2}, x^{\prime 3}\right)$, where $B:=\left\{B_{m j}\right\}_{m, j=0,1,2,3} \in \mathbb{R}^{4 \times 4}$ and $\left(z^{0}, z^{1}, z^{2}, z^{3}\right) \in \mathbb{R}^{4}$ in (2.58) are independent on the argument $s$ (Obviously such a coordinate change is necessarily a Lorentz's transformation).

As a direct consequence of Proposition 2.3 we deduce two following Corollaries:

Corollary 2.6. - Given fixed vector $\left(w_{0}, w_{1}, w_{2}, w_{3}\right) \in \mathbb{R}^{4}$, satisfying (2.73), assume that the change of coordinate system $\left(x^{0}, x^{1}, x^{2}, x^{3}\right) \rightarrow\left(x^{\prime 0}, x^{\prime 1}, x^{\prime 2}, x^{\prime 3}\right)$ is of the class $P L\left(w_{0}, w_{1}, w_{2}, w_{3}\right)$. Then the constant vector $\left(w_{0}^{\prime}, w_{1}^{\prime}, w_{2}^{\prime}, w_{3}^{\prime}\right) \in \mathbb{R}^{4}$, given by (2.75) satisfies (2.74) and the above coordinate change $\left(x^{0}, x^{1}, x^{2}, x^{3}\right) \rightarrow\left(x^{\prime 0}, x^{\prime 1}, x^{\prime 2}, x^{\prime 3}\right)$ is of the class $P L\left(\left(w_{0}, w_{1}, w_{2}, w_{3}\right) ;\left(w_{0}^{\prime}, w_{1}^{\prime}, w_{2}^{\prime}, w_{3}^{\prime}\right)\right)$.

- Given a Lorentz's transformation $\left(x^{0}, x^{1}, x^{2}, x^{3}\right) \rightarrow\left(x^{\prime 0}, x^{\prime 1}, x^{\prime 2}, x^{\prime 3}\right)$, there exist two fixed constant vectors $\left(w_{0}, w_{1}, w_{2}, w_{3}\right) \in \mathbb{R}^{4}$ and $\left(w_{0}^{\prime}, w_{1}^{\prime}, w_{2}^{\prime}, w_{3}^{\prime}\right) \in \mathbb{R}^{4}$, satisfying (2.73) and (2.74), such that the above of change coordinates $\left(x^{0}, x^{1}, x^{2}, x^{3}\right) \rightarrow\left(x^{\prime 0}, x^{\prime 1}, x^{\prime 2}, x^{\prime 3}\right)$ is of the class $L\left(\left(w_{0}, w_{1}, w_{2}, w_{3}\right) ;\left(w_{0}^{\prime}, w_{1}^{\prime}, w_{2}^{\prime}, w_{3}^{\prime}\right)\right)$.

Corollary 2.7. Consider a standard kinematical Lorentz's structure with global time $\varphi$ on $\mathbb{R}^{4}$ together with the corresponding tensor of three-dimensional Geometry $\left\{\Lambda^{m n}\right\}_{m, n=0,1,2,3}$. Next consider the first cartesian, with respect to $\left\{\Lambda^{m n}\right\}_{m, n=0,1,2,3}$ and $\varphi$, coordinate system in $\mathbb{R}^{4}$ and the second general coordinate system in $\mathbb{R}^{4}$. Then, the second coordinate system is also cartesian if and only if the change of the first coordinate system to the second one is of class $P L((1,0,0,0) ;(1,0,0,0))$.

Proposition 2.4. Given two fixed constant vectors $\left(w_{0}, w_{1}, w_{2}, w_{3}\right) \in \mathbb{R}^{4}$ and $\left(w_{0}^{\prime}, w_{1}^{\prime}, w_{2}^{\prime}, w_{3}^{\prime}\right) \in \mathbb{R}^{4}$, satisfying

$$
\sum_{j=0}^{3} \sum_{m=0}^{3} M^{j m} w_{j} w_{m}=1
$$

and

$$
\sum_{j=0}^{3} \sum_{m=0}^{3} M^{j m} w_{j}^{\prime} w_{m}^{\prime}=1
$$


assume that the change of coordinate system $\left(x^{0}, x^{1}, x^{2}, x^{3}\right) \rightarrow\left(x^{\prime 0}, x^{\prime 1}, x^{\prime 2}, x^{\prime 3}\right)$ is of the class $P L\left(\left(w_{0}, w_{1}, w_{2}, w_{3}\right) ;\left(w_{0}^{\prime}, w_{1}^{\prime}, w_{2}^{\prime}, w_{3}^{\prime}\right)\right)$. Then the inverse change of variables $\left(x^{\prime 0}, x^{\prime 1}, x^{\prime 2}, x^{\prime 3}\right) \rightarrow$ $\left(x^{0}, x^{1}, x^{2}, x^{3}\right)$ is of the class $\operatorname{PL}\left(\left(w_{0}^{\prime}, w_{1}^{\prime}, w_{2}^{\prime}, w_{3}^{\prime}\right) ;\left(w_{0}, w_{1}, w_{2}, w_{3}\right)\right)$.

As a direct consequence of Lemma 10.1 we deduce:

Proposition 2.5. Given three fixed constant vectors $\left(w_{0}, w_{1}, w_{2}, w_{3}\right) \in \mathbb{R}^{4},\left(w_{0}^{\prime}, w_{1}^{\prime}, w_{2}^{\prime}, w_{3}^{\prime}\right) \in \mathbb{R}^{4}$ and $\left(w_{0}^{\prime \prime}, w_{1}^{\prime \prime}, w_{2}^{\prime \prime}, w_{3}^{\prime \prime}\right) \in \mathbb{R}^{4}$, satisfying

$$
\left\{\begin{array}{l}
\sum_{j=0}^{3} \sum_{m=0}^{3} M^{j m} w_{j} w_{m}=1 \\
\sum_{j=0}^{3} \sum_{m=0}^{3} M^{j m} w_{j}^{\prime} w_{m}^{\prime}=1 \\
\sum_{j=0}^{3} \sum_{m=0}^{3} M^{j m} w_{j}^{\prime \prime} w_{m}^{\prime \prime}=1,
\end{array}\right.
$$

assume that that the change of coordinate system $\left(x^{0}, x^{1}, x^{2}, x^{3}\right) \rightarrow\left(x^{\prime 0}, x^{\prime 1}, x^{\prime 2}, x^{\prime 3}\right)$ belongs to the class $P L\left(\left(w_{0}, w_{1}, w_{2}, w_{3}\right) ;\left(w_{0}^{\prime}, w_{1}^{\prime}, w_{2}^{\prime}, w_{3}^{\prime}\right)\right)$ and another change of coordinate system $\left(x^{\prime 0}, x^{\prime 1}, x^{\prime 2}, x^{\prime 3}\right) \rightarrow$ $\left(x^{\prime \prime 0}, x^{\prime \prime 1}, x^{\prime \prime 2}, x^{\prime \prime 3}\right)$, belonging to the class $P L\left(\left(w_{0}^{\prime}, w_{1}^{\prime}, w_{2}^{\prime}, w_{3}^{\prime}\right) ;\left(w_{0}^{\prime \prime}, w_{1}^{\prime \prime}, w_{2}^{\prime \prime}, w_{3}^{\prime \prime}\right)\right)$. Then, the composition of the above two changes of coordinate systems:

$\left(x^{0}, x^{1}, x^{2}, x^{3}\right) \rightarrow\left(x^{\prime \prime 0}, x^{\prime \prime 1}, x^{\prime \prime 2}, x^{\prime \prime 3}\right)=\left(x^{0}, x^{1}, x^{2}, x^{3}\right) \rightarrow\left(x^{\prime 0}, x^{\prime 1}, x^{\prime 2}, x^{\prime 3}\right) \rightarrow\left(x^{\prime \prime 0}, x^{\prime \prime 1}, x^{\prime \prime 2}, x^{\prime \prime 3}\right)$

belongs to the class $P L\left(\left(w_{0}, w_{1}, w_{2}, w_{3}\right) ;\left(w_{0}^{\prime \prime}, w_{1}^{\prime \prime}, w_{2}^{\prime \prime}, w_{3}^{\prime \prime}\right)\right)$.

As a direct consequence of the Corollary 10.1 and Proposition 2.5 we deduce:

Proposition 2.6. Given three fixed constant vectors $\left(w_{0}, w_{1}, w_{2}, w_{3}\right) \in \mathbb{R}^{4},\left(w_{0}^{\prime}, w_{1}^{\prime}, w_{2}^{\prime}, w_{3}^{\prime}\right) \in \mathbb{R}^{4}$ and $\left(\hat{w}_{0}, \hat{w}_{1}, \hat{w}_{2}, \hat{w}_{3}\right) \in \mathbb{R}^{4}$, satisfying

$$
\left\{\begin{array}{l}
\sum_{j=0}^{3} \sum_{m=0}^{3} M^{j m} w_{j} w_{m}=1 \\
\sum_{j=0}^{3} \sum_{m=0}^{3} M^{j m} w_{j}^{\prime} w_{m}^{\prime}=1 \\
\sum_{j=0}^{3} \sum_{m=0}^{3} M^{j m} \hat{w}_{j} \hat{w}_{m}=1,
\end{array}\right.
$$

the change of coordinate system $\left(x^{0}, x^{1}, x^{2}, x^{3}\right) \rightarrow\left(x^{\prime \prime \prime}, x^{\prime \prime \prime 1}, x^{\prime \prime \prime 2}, x^{\prime \prime \prime 3}\right)$ belongs to the class $P L\left(\left(w_{0}, w_{1}, w_{2}, w_{3}\right) ;\left(w_{0}^{\prime}, w_{1}^{\prime}, w_{2}^{\prime}, w_{3}^{\prime}\right)\right)$ if and only if there exists three other changes of coordinate system $\left(x^{0}, x^{1}, x^{2}, x^{3}\right) \rightarrow\left(x^{\prime 0}, x^{\prime 1}, x^{\prime 2}, x^{\prime 3}\right)$, belonging to the class $L\left(\left(w_{0}, w_{1}, w_{2}, w_{3}\right) ;\left(\hat{w}_{0}, \hat{w}_{1}, \hat{w}_{2}, \hat{w}_{3}\right)\right)$, $\left(x^{\prime 0}, x^{\prime 1}, x^{\prime 2}, x^{\prime 3}\right) \rightarrow\left(x^{\prime \prime 0}, x^{\prime \prime 1}, x^{\prime \prime 2}, x^{\prime \prime 3}\right)$, belonging to the class $P L\left(\left(\hat{w}_{0}, \hat{w}_{1}, \hat{w}_{2}, \hat{w}_{3}\right) ;\left(\hat{w}_{0}, \hat{w}_{1}, \hat{w}_{2}, \hat{w}_{3}\right)\right)$ and $\left(x^{\prime \prime \prime}, x^{\prime \prime 1}, x^{\prime \prime 2}, x^{\prime \prime 3}\right) \rightarrow\left(x^{\prime \prime \prime 0}, x^{\prime \prime \prime 1}, x^{\prime \prime \prime 2}, x^{\prime \prime \prime 3}\right)$, belonging to the class $L\left(\left(\left(\hat{w}_{0}, \hat{w}_{1}, \hat{w}_{2}, \hat{w}_{3}\right) ;\left(w_{0}^{\prime}, w_{1}^{\prime}, w_{2}^{\prime}, w_{3}^{\prime}\right)\right)\right.$, so that the original transformation $\left(x^{0}, x^{1}, x^{2}, x^{3}\right) \rightarrow\left(x^{\prime \prime \prime \prime}, x^{\prime \prime \prime 1}, x^{\prime \prime \prime 2}, x^{\prime \prime \prime}\right)$ is a composition of the above three changes of coordinate systems:

$$
\begin{aligned}
& \left(x^{0}, x^{1}, x^{2}, x^{3}\right) \rightarrow\left(x^{\prime \prime \prime \prime}, x^{\prime \prime \prime 1}, x^{\prime \prime \prime 2}, x^{\prime \prime \prime 3}\right)= \\
& \left(x^{0}, x^{1}, x^{2}, x^{3}\right) \rightarrow\left(x^{\prime 0}, x^{\prime 1}, x^{\prime 2}, x^{\prime 3}\right) \rightarrow\left(x^{\prime \prime 0}, x^{\prime \prime 1}, x^{\prime \prime 2}, x^{\prime \prime 3}\right) \rightarrow\left(x^{\prime \prime \prime 0}, x^{\prime \prime \prime 1}, x^{\prime \prime \prime 2}, x^{\prime \prime \prime 3}\right) .
\end{aligned}
$$


As a direct consequence of Proposition 2.6 we deduce the following:

Corollary 2.8. Given two fixed constant vectors $\left(w_{0}, w_{1}, w_{2}, w_{3}\right) \in \mathbb{R}^{4},\left(w_{0}^{\prime}, w_{1}^{\prime}, w_{2}^{\prime}, w_{3}^{\prime}\right) \in \mathbb{R}^{4}$, satisfying

$$
\left\{\begin{array}{l}
\sum_{j=0}^{3} \sum_{m=0}^{3} M^{j m} w_{j} w_{m}=1 \\
\sum_{j=0}^{3} \sum_{m=0}^{3} M^{j m} w_{j}^{\prime} w_{m}^{\prime}=1,
\end{array}\right.
$$

the change of coordinate system $\left(x^{0}, x^{1}, x^{2}, x^{3}\right) \rightarrow\left(x^{\prime \prime \prime}, x^{\prime \prime \prime 1}, x^{\prime \prime \prime 2}, x^{\prime \prime \prime 3}\right)$ belongs to the class $\operatorname{PL}\left(\left(w_{0}, w_{1}, w_{2}, w_{3}\right) ;\left(w_{0}^{\prime}, w_{1}^{\prime}, w_{2}^{\prime}, w_{3}^{\prime}\right)\right)$ if and only if there exists three other changes of coordinate system $\left(x^{0}, x^{1}, x^{2}, x^{3}\right) \rightarrow\left(x^{\prime 0}, x^{\prime 1}, x^{\prime 2}, x^{\prime 3}\right)$, belonging to the class $L\left(\left(w_{0}, w_{1}, w_{2}, w_{3}\right) ;(1,0,0,0)\right)$, $\left(x^{\prime 0}, x^{\prime 1}, x^{\prime 2}, x^{\prime 3}\right) \rightarrow\left(x^{\prime \prime 0}, x^{\prime \prime 1}, x^{\prime \prime 2}, x^{\prime \prime 3}\right)$, belonging to the class $P L((1,0,0,0) ;(1,0,0,0))$ and $\left(x^{\prime \prime 0}, x^{\prime \prime 1}, x^{\prime \prime 2}, x^{\prime \prime 3}\right) \rightarrow\left(x^{\prime \prime \prime 0}, x^{\prime \prime \prime 1}, x^{\prime \prime \prime 2}, x^{\prime \prime \prime 3}\right)$, belonging to the class $L\left(\left((1,0,0,0) ;\left(w_{0}^{\prime}, w_{1}^{\prime}, w_{2}^{\prime}, w_{3}^{\prime}\right)\right)\right.$, so that the original transformation $\left(x^{0}, x^{1}, x^{2}, x^{3}\right) \rightarrow\left(x^{\prime \prime \prime}, x^{\prime \prime \prime}, x^{\prime \prime \prime 2}, x^{\prime \prime \prime}\right)$ is a composition of the above three changes of coordinate systems:

$$
\begin{aligned}
& \left(x^{0}, x^{1}, x^{2}, x^{3}\right) \rightarrow\left(x^{\prime \prime \prime}, x^{\prime \prime \prime 1}, x^{\prime \prime \prime 2}, x^{\prime \prime \prime 3}\right) \\
& \quad\left(x^{0}, x^{1}, x^{2}, x^{3}\right) \rightarrow\left(x^{\prime 0}, x^{\prime 1}, x^{\prime 2}, x^{\prime 3}\right) \rightarrow\left(x^{\prime \prime 0}, x^{\prime \prime 1}, x^{\prime \prime 2}, x^{\prime \prime 3}\right) \rightarrow\left(x^{\prime \prime \prime 0}, x^{\prime \prime \prime}, x^{\prime \prime \prime 2}, x^{\prime \prime \prime 3}\right) .
\end{aligned}
$$

\subsection{Kinematical Lorentz's structure with global time}

Definition 2.15. We say that a contravariant pseudo-metric $\left\{K^{m n}\right\}_{m, n=0,1,2,3}$ on $\mathbb{R}^{4}$ is flat if the curvature-tensor of this pseudo-metric vanishes. In other words, at every point in $\mathbb{R}^{4}$ we have

$$
\begin{aligned}
& \left\{R_{k m n}^{j}\right\}_{K}:= \\
& \begin{aligned}
\frac{\partial}{\partial x^{m}}\left(\left\{\Gamma_{k n}^{j}\right\}_{K}\right)-\frac{\partial}{\partial x^{n}}\left(\left\{\Gamma_{k m}^{j}\right\}_{K}\right)+\sum_{d=0}^{3}\left\{\Gamma_{k n}^{d}\right\}_{K}\left\{\Gamma_{d m}^{j}\right\}_{K}-\sum_{d=0}^{3}\left\{\Gamma_{k m}^{d}\right\}_{K}\left\{\Gamma_{n d}^{j}\right\}_{K}=0 \\
\forall j, k, m, n=0,1,2,3,
\end{aligned}
\end{aligned}
$$

where the Christoffel Symbols $\left\{\Gamma_{k j}^{m}\right\}_{K}$ are defined by (2.25). It is well known that the equality in (2.83) is independent on the chosen coordinate system.

Remark 2.4. Obviously, if given a pseudo-metric $\left\{K^{m n}\right\}_{m, n=0,1,2,3}$, there exists an inertial coordinate

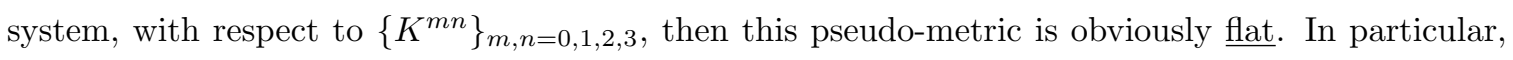
if there exists some coordinate system where matrix $\left\{K^{m n}\right\}_{m, n=0,1,2,3}$ has a form of

$$
\begin{cases}K^{00}=1 & \\ K^{0 j}=K^{j 0}=0 & \forall j=1,2,3 \\ K^{j m}:=-\delta_{j m} & \forall j, m=1,2,3\end{cases}
$$

then a contravariant pseudo-metric $\left\{K^{m n}\right\}_{m, n=0,1,2,3}$ on $\mathbb{R}^{4}$ is flat. 
Definition 2.16. Given a generalized-Lorentz's structure with global time on $\mathbb{R}^{4}$, formed by a strongly correlating contravariant pseudo-metric $\left\{K^{m n}\right\}_{m, n=0,1,2,3}$ and a global time $\varphi$, we say that this generalized-Lorentz's structure with global time is kinematical, if pseudo-metric $\left\{K^{m n}\right\}_{m, n=0,1,2,3}$ is flat and, at the same time, the tensor of the covariant derivatives of the covector $\left(\frac{\partial \varphi}{\partial x^{0}}, \frac{\partial \varphi}{\partial x^{1}}, \frac{\partial \varphi}{\partial x^{2}}, \frac{\partial \varphi}{\partial x^{3}}\right)$ vanishes:

$$
\left\{\delta_{j}\left(\frac{\partial \varphi}{\partial x^{k}}\right)\right\}_{K}=0 \quad \forall k, j=0,1,2,3 .
$$

Remark 2.5. Consider a standard kinematical Lorentz's structure with global time on $\mathbb{R}^{4}$, formed by a pseudo-metric $\left\{K^{m n}\right\}_{m, n=0,1,2,3}$ and a global time $\varphi$, so that there exists some coordinate system where matrix $\left\{K^{m n}\right\}_{m, n=0,1,2,3}$ has a form of

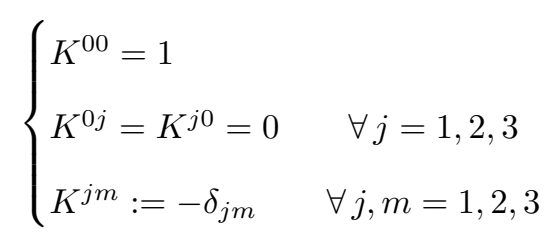

and at the same time the covector of time direction for $\varphi$ has a form

$$
\left(\frac{\partial \varphi}{\partial x^{0}}, \frac{\partial \varphi}{\partial x^{1}}, \frac{\partial \varphi}{\partial x^{2}}, \frac{\partial \varphi}{\partial x^{3}}\right)\left(x^{0}, x^{1}, x^{2}, x^{3}\right)=(1,0,0,0) \quad \forall\left(x^{0}, x^{1}, x^{2}, x^{3}\right) \in \mathbb{R}^{4} .
$$

Then, we obviously obtain that this standard kinematical Lorentz's structure with global time is kinematical in the sense of Definition 2.16. On the other hand, given an arbitrary kinematical Lorentz's structure with global time, one can prove that every point in $\mathbb{R}^{4}$ has at least some neighborhood, in which (2.86) and (2.87) hold in some coordinate system.

\subsection{Kinematical and Dynamical generalized-Lorentz structures with time direction}

We postulate that all real physical processes appear in some valid contravariant pseudo-metric $\left\{K^{m n}\right\}_{m, n=0,1,2,3}$, describing the generalized gravity field, weakly correlated with some covector of time direction $\left(w_{0}, w_{1}, w_{2}, w_{3}\right)$. Furthermore, we distinguish two types of generalized gravity. The first type is the fictitious gravity which we call inertia. This type of gravity depends only on the flat geometry of empty space-time via the choice of specific coordinate system and it is independent on the surrounding real matter consisting of gravitational masses or other real physical fields. The second type of the gravity is the genuine (real) gravity, which depends essentially on the real physical matter, especially on gravitational masses. We assume that this type of gravity vanishes away from essential gravitational masses and strong real physical fields. Then we state the First Law of the Newton as the following:

Proposition 2.7. [Axiom I] In the parts of the space-time where we observes the absence of genuine gravity, and in particular away from essential real physical bodies and fields, we have

$$
K^{m n}=J^{m n} \quad \forall m, n=0,1,2,3,
$$


and

$$
\left(w_{0}, w_{1}, w_{2}, w_{3}\right)=\left(\frac{\partial \varphi}{\partial x^{0}}, \frac{\partial \varphi}{\partial x^{1}}, \frac{\partial \varphi}{\partial x^{2}}, \frac{\partial \varphi}{\partial x^{3}}\right),
$$

where the strongly-correlated contravariant pseudo-metric $\left\{J^{m n}\right\}_{m, n=0,1,2,3}$ and the global time $\varphi$ form the standard kinematical Lorentz's structure with global time on $\mathbb{R}^{4}$, as defined in Definition 2.7.

Remark 2.6. We remind, that by Definition 2.7, there exists some coordinate system where matrix $\left\{J^{m n}\right\}_{m, n=0,1,2,3}$ has a form of

$$
\begin{cases}J^{00}=1 & \\ J^{0 j}=J^{j 0}=0 & \forall j=1,2,3 \\ J^{j m}:=-\delta_{j m} & \forall j, m=1,2,3\end{cases}
$$

and at the same coordinate system the covector of time direction for the global time $\varphi$ has a form

$$
\left(\frac{\partial \varphi}{\partial x^{0}}, \frac{\partial \varphi}{\partial x^{1}}, \frac{\partial \varphi}{\partial x^{2}}, \frac{\partial \varphi}{\partial x^{3}}\right)\left(x^{0}, x^{1}, x^{2}, x^{3}\right)=(1,0,0,0) \quad \forall\left(x^{0}, x^{1}, x^{2}, x^{3}\right) \in \mathbb{R}^{4} .
$$

We remind that this particular system is called kinematically preferable and it is unique, up to equivalence. Furthermore, we define the kinematical tensor of three-dimensional Geometry $\left\{\Theta^{m n}\right\}_{m, n=0,1,2,3}$, given by

$$
\Theta^{m n}:=\left(\sum_{j=0}^{3} J^{m j} \frac{\partial \varphi}{\partial x^{j}}\right)\left(\sum_{j=0}^{3} J^{n j} \frac{\partial \varphi}{\partial x^{j}}\right)-J^{m n} \quad \forall m, n=0,1,2,3,
$$

where

$$
\left(\sum_{j=0}^{3} J^{0 j} \frac{\partial \varphi}{\partial x^{j}}, \sum_{j=0}^{3} J^{1 j} \frac{\partial \varphi}{\partial x^{j}}, \sum_{j=0}^{3} J^{2 j} \frac{\partial \varphi}{\partial x^{j}}, \sum_{j=0}^{3} J^{3 j} \frac{\partial \varphi}{\partial x^{j}}\right)
$$

is the contravariant vector of inertia. In particular, in the kinematically preferable coordinate system, where (2.90) and (2.91) holds we have

$$
\left\{\begin{array}{l}
\Theta^{00}=0 \\
\Theta^{0 j}=\Theta^{j 0}=0 \quad \forall j=1,2,3 \\
\Theta^{j m}:=\delta_{j m} \quad \forall j, m=1,2,3 .
\end{array}\right.
$$

We also remind that, given arbitrary coordinate system, it is called cartesian if in this system we have simultaneously (2.94) and (2.91) but, we do not necessary have (2.90). On the other hand, given arbitrary coordinate system, it is called Lorentzian if in this system we have (2.90) but, we do not necessary have (2.94) or (2.91). Finally, given arbitrary coordinate system, it is inertial, if we can get it from kinematically preferable coordinate system by a linear transformation. We also remind, that we obtain a coordinate system which is simultaneously cartesian and inertial from another such system by Galilean transformations. On the other hand, we obtain a coordinate system which is 
simultaneously Loretzian and inertial from another such system by Lorentz's transformations. The unique, up to equivalence, coordinate system which is simultaneously cartesian and Loretzian is a kinematically preferable coordinate system.

\subsection{Lagrangian of the motion of a classical point particle in a given pseudo-metric with time direction}

Definition 2.17. Consider a classical point particle with the inertial mass $m$ and the charge $\sigma$ moving in the generalized-gravitational field, given by a contravariant pseudo-metric $\left\{K^{m n}\right\}_{m, n=0,1,2,3}$, weakly correlated with a four-covector of the time direction $\left(w_{0}, w_{1}, w_{2}, w_{3}\right)$, and influenced by the electromagnetic field with the four-covector of the electromagnetic potential $\left(A_{0}, A_{1}, A_{2}, A_{3}\right)$. Next assume that $\chi(s):=\left(\chi^{0}(s), \chi^{1}(s), \chi^{2}(s), \chi^{3}(s)\right):[a, b] \rightarrow \mathbb{R}^{4} \in \mathbb{R}^{4}$ is a four-dimensional space-time trajectory of the particle, parameterized by some general scalar parameter $s \in[a, b]$ (including the cases where $a=-\infty$ and/or $b=+\infty$ ). Then we say that the parameter of the trajectory $s$ is proper with respect the time direction $\left(w_{0}, w_{1}, w_{2}, w_{3}\right)$, if we have

$$
\sum_{j=0}^{3} w_{j}(\chi(s)) \frac{d \chi^{j}}{d s}(s)>0 \quad \forall s \in[a, b] .
$$

Next, given a proper parametrization $s$, we define the contravariant four-vector of the velocity of the particle $\left(u^{0}, u^{1}, u^{2}, u^{3}\right)(s)$, with respect to the time direction $\left(w_{0}, w_{1}, w_{2}, w_{3}\right)$, by the following

$$
u^{j}(s)=\left(\sum_{k=0}^{3} w_{k}(\chi(s)) \frac{d \chi^{k}}{d s}(s)\right)^{-1} \frac{d \chi^{j}}{d s}(s) \quad \forall j=0,1,2,3 \quad \forall s \in[a, b],
$$

so that

$$
\sum_{j=0}^{3} w_{j}(\chi(s)) u^{j}(s)=1 \quad \forall s \in[a, b]
$$

(It is obvious that the definition of $\left(u^{0}, u^{1}, u^{2}, u^{3}\right)$ in (2.96) is independent on the choice of the proper parametrization $s$ ). Moreover, given an arbitrary contravariant four-vector field $\left(f^{0}, f^{1}, f^{2}, f^{3}\right)$, we say that this field is a speed-like four-vector field, with respect to the time direction $\left(w_{0}, w_{1}, w_{2}, w_{3}\right)$, if we have

$$
\sum_{j=0}^{3} w_{j}\left(x^{0}, x^{1}, x^{2}, x^{3}\right) f^{j}\left(x^{0}, x^{1}, x^{2}, x^{3}\right)=1 \quad \forall\left(x^{0}, x^{1}, x^{2}, x^{3}\right) \in \mathbb{R}^{4} .
$$

Remark 2.7. Note here the difference in our notation with respect to the usual Special and General Relativity: in the usual Theory of Relativity the notation of the four-velocity of the particle is booked for the normalized contravariant four-vector $\left(\hat{u}^{0}, \hat{u}^{1}, \hat{u}^{2}, \hat{u}^{3}\right)(s)$, given by

$$
\begin{aligned}
\hat{u}^{j}(s):= & \left(\sum_{m=0}^{3} \sum_{n=0}^{3} K_{m n}(\chi(s)) \frac{d \chi^{m}}{d s}(s) \frac{d \chi^{n}}{d s}(s)\right)^{-\frac{1}{2}} \frac{d \chi^{j}}{d s}(s)= \\
& \left(\sum_{m=0}^{3} \sum_{n=0}^{3} K_{m n}(\chi(s)) u^{m}(s) u^{n}(s)\right)^{-\frac{1}{2}} u^{j}(s) \quad \forall j=0,1,2,3 \quad \forall s \in[a, b],
\end{aligned}
$$


while, in the present paper by the name four-velocity we denote the contravariant four-vector $\left(u^{0}, u^{1}, u^{2}, u^{3}\right)(s)$, given by $(2.96)$.

Furthermore, given a proper parametrization $s$, consider the Lagrangian of motion of this particle given by the following (in the Gaussian unit system)

$$
\begin{gathered}
L_{\mathcal{G}}(\chi)=\int_{a}^{b}\left\{-m \mathcal{G}\left(\sum_{j=0}^{3} \sum_{k=0}^{3} K_{j k}(\chi(s)) u^{j}(s) u^{k}(s)\right)\right\}\left(\sum_{j=0}^{3} w_{j}(\chi(s)) \frac{d \chi^{j}}{d s}\right) d s \\
+\int_{a}^{b}\left\{-\sum_{j=0}^{3} \sigma A_{j}(\chi(s)) \frac{d \chi^{j}}{d s}\right\} d s= \\
\int_{a}^{b}\left\{-m \mathcal{G}\left(\sum_{j=0}^{3} \sum_{k=0}^{3} K_{j k}(\chi(s)) u^{j}(s) u^{k}(s)\right)-\sum_{j=0}^{3} \sigma u^{j}(s) A_{j}(\chi(s))\right\}\left(\sum_{j=0}^{3} w_{j}(\chi(s)) \frac{d \chi^{j}}{d s}\right) d s,
\end{gathered}
$$

where $\left\{K_{m n}\right\}_{m, n=0,1,2,3}$ is the inverse to $\left\{K^{m n}\right\}_{m, n=0,1,2,3}$ covariant pseudo-metric and $\mathcal{G}(\tau): \mathbb{R} \rightarrow \mathbb{R}$ is some fixed function. Obviously, the functional in (2.100) is independent on the choice of the proper parametrization. We have the following two important particular cases:

- The case of relativistic particle where $\mathcal{G}(\tau):=\sqrt{\tau}-1$ in $(2.100)$.

- The case of non-relativistic particle where $\mathcal{G}(\tau):=\frac{1}{2}(\tau-1)$ in $(2.100)$.

In both cases $\mathcal{G}(1)=0$. Moreover, we have

$$
\sqrt{\tau}-1=\frac{1}{2}(\tau-1)+\mathcal{O}\left((\tau-1)^{2}\right) .
$$

In the first relativistic case we simplify (2.100) as:

$$
\begin{aligned}
L_{1}(\chi)=\int_{a}^{b}\left\{-m \sqrt{\sum_{j=0}^{3} \sum_{k=0}^{3} K_{j k}(\chi(s))} \frac{d \chi^{j}}{d s} \frac{d \chi^{k}}{d s}\right\} d s \\
+\int_{a}^{b}\left\{-\sum_{j=0}^{3} \sigma A_{j}(\chi(s)) \frac{d \chi^{j}}{d s}+\sum_{j=0}^{3} m w_{j}(\chi(s)) \frac{d \chi^{j}}{d s}\right\} d s .
\end{aligned}
$$

On the other hand, in the second, non-relativistic case we simplify (2.100) as:

$$
\begin{gathered}
L_{2}(\chi)=\int_{a}^{b}\left\{-\frac{m}{2} \frac{\left(\sum_{j=0}^{3} \sum_{k=0}^{3} K_{j k}(\chi(s)) \frac{d \chi^{j}}{d s} \frac{d \chi^{k}}{d s}\right)}{\left(\sum_{j=0}^{3} w_{j}(\chi(s)) \frac{d \chi^{j}}{d s}\right)}\right\} d s \\
+\int_{a}^{b}\left\{-\sum_{j=0}^{3} \sigma A_{j}(\chi(s)) \frac{d \chi^{j}}{d s}+\sum_{j=0}^{3} \frac{m}{2} w_{j}(\chi(s)) \frac{d \chi^{j}}{d s}\right\} d s .
\end{gathered}
$$

Definition 2.18. Given two events on the trajectory of the motion of the particle $\chi\left(s_{1}\right) \in \mathbb{R}^{4}$ and $\chi\left(s_{2}\right) \in \mathbb{R}^{4}$, with parameters of the chosen proper parametrization $s_{1}$ and $s_{2}$ respectively, we define 
the interval of time that passed from the event $\chi\left(s_{1}\right)$ to event $\chi\left(s_{2}\right)$, corresponding to the time direction $\left(w_{0}, w_{1}, w_{2}, w_{3}\right)$, as:

$$
\tau\left(\chi\left(s_{1}\right), \chi\left(s_{2}\right)\right):=\int_{s_{1}}^{s_{2}}\left(\sum_{j=0}^{3} w_{j}(\chi(s)) \frac{d \chi^{j}}{d s}\right) d s .
$$

Then obviously the interval of time is independent on the proper parametrization of the trajectory. Moreover if we have

$$
\left(w_{0}, w_{1}, w_{2}, w_{3}\right)=\left(\frac{\partial \psi}{\partial x^{0}}, \frac{\partial \psi}{\partial x^{1}}, \frac{\partial \psi}{\partial x^{2}}, \frac{\partial \psi}{\partial x^{3}}\right) \quad \forall\left(x^{0}, x^{1}, x^{2}, x^{3}\right) \in \mathbb{R}^{4},
$$

where $\psi$ is some global time (which is obviously weakly correlated with $\left\{K^{m n}\right\}_{m, n=0,1,2,3}$ ), then, inserting (2.105) into (2.104) we obviously deduce

$$
\tau\left(\chi\left(s_{1}\right), \chi\left(s_{2}\right)\right)=\psi\left(\chi\left(s_{2}\right)\right)-\psi\left(\chi\left(s_{1}\right)\right) .
$$

So, in the later case the interval of time between two events is the difference in global time between the second and the first event and therefore, this interval is independent on the trajectory itself, but depends only on the two ending points of the trajectory. Note also that in the general case the quantity

$$
\tau(s):=\tau(\chi(s), \chi(a)) \quad \forall s \in[a, b],
$$

can be chosen as a preferable parameter of the trajectory of the motion and we rewrite the relativistic Lagrangian in (2.102) as:

$$
L_{1}(\chi)=\int_{0}^{\tau(b)}\left\{m-m\left(\sqrt{\sum_{j=0}^{3} \sum_{k=0}^{3} K_{j k}(\chi(\tau)) \frac{d \chi^{j}}{d \tau} \frac{d \chi^{k}}{d \tau}}\right)-\sum_{j=0}^{3} \sigma A_{j}(\chi(\tau)) \frac{d \chi^{j}}{d \tau}\right\} d \tau
$$

and we rewrite the non-relativistic Lagrangian in (2.103) as

$$
L_{2}(\chi)=\int_{0}^{\tau(b)}\left\{\frac{m}{2}-\frac{m}{2}\left(\sum_{j=0}^{3} \sum_{k=0}^{3} K_{j k}(\chi(\tau)) \frac{d \chi^{j}}{d \tau} \frac{d \chi^{k}}{d \tau}\right)-\sum_{j=0}^{3} \sigma A_{j}(\chi(\tau)) \frac{d \chi^{j}}{d \tau}\right\} d \tau .
$$

Moreover, in the case of general $\mathcal{G}$, we rewrite the general Lagrangian in (2.100) as:

$$
L_{\mathcal{G}}(\chi)=\int_{0}^{\tau(b)}\left\{-m \mathcal{G}\left(\sum_{j=0}^{3} \sum_{k=0}^{3} K_{j k}(\chi(\tau)) \frac{d \chi^{j}}{d \tau} \frac{d \chi^{k}}{d \tau}\right)-\sum_{j=0}^{3} \sigma A_{j}(\chi(\tau)) \frac{d \chi^{j}}{d \tau}\right\} d \tau,
$$

and the velocity satisfies

$$
u^{j}(\tau)=\frac{d \chi^{j}}{d \tau}(\tau) \quad \forall j=0,1,2,3 \quad \forall s \in[0, \tau(b)] .
$$

Furthermore, we postulate the Second Law of Newton:

Proposition 2.8. [Axiom II] The motion of a classical point particle with inertial mass $m$ and charge $\sigma$ is described by the Lagrangian (2.100), where we consider either $\mathcal{G}(\tau):=\sqrt{\tau}-1$ (the relativistic case) or $\mathcal{G}(\tau):=\frac{1}{2}(\tau-1)$ (a non-relativistic approximation). 
As a consequence of the first and the second laws of Newton we deduce the following.

Corollary 2.9. [The law of inertia] Assume that either $\mathcal{G}(\tau):=\sqrt{\tau}-1$ or $\mathcal{G}(\tau):=\frac{1}{2}(\tau-1)$ in $(2.100)$. Then, in the absence of genuine gravitational field, the electromagnetical or any other physical field, given an arbitrary inertial coordinate system, the trajectory of the classical point particle in this coordinate system is a direct-line in $\mathbb{R}^{4}$. Note that the coordinate system must be inertial, but not necessary cartesian or Lorentzian.

\subsection{Lagrangian of the electromagnetic field in a given pseudo-metric}

Definition 2.19. Consider a contravariant pseudo-metric $K:=\left\{K^{m n}\right\}_{m, n=0,1,2,3}$ and let $A=$ $\left(A_{0}, A_{1}, A_{2}, A_{3}\right):=\left(A_{0}, A_{1}, A_{2}, A_{3}\right)\left(x^{0}, x^{1}, x^{2}, x^{3}\right)$ be the four-covector of the electromagnetic potential and $j=\left(j^{0}, j^{1}, j^{2}, j^{3}\right):=\left(j^{0}, j^{1}, j^{2}, j^{3}\right)\left(x^{0}, x^{1}, x^{2}, x^{3}\right)$ be the contravariant four-vector of the four-current. As usual, we consider the Lagrangian-density of the electromagnetic field given by the following (in the Gaussian unit system):

$$
\begin{aligned}
& \mathcal{L}_{e}\left(\left(A_{0}, A_{1}, A_{2}, A_{3}\right),\left\{K^{m n}\right\}_{m, n=0,1,2,3}\right):= \\
& \quad \frac{1}{4 \pi}\left(-\sum_{n=0}^{3} \sum_{k=0}^{3} \sum_{m=0}^{3} \sum_{p=0}^{3} \frac{1}{4} K^{m n} K^{p k}\left(\frac{\partial A_{p}}{\partial x^{m}}-\frac{\partial A_{m}}{\partial x^{p}}\right)\left(\frac{\partial A_{k}}{\partial x^{n}}-\frac{\partial A_{n}}{\partial x^{k}}\right)-\sum_{k=0}^{3} 4 \pi j^{k} A_{k}\right)
\end{aligned}
$$

(we use the dynamical pseudo-metric $\left\{K^{m n}\right\}_{m, n=0,1,2,3}$ in this definition since it is well known that the electromagnetic field is dependent on the genuine gravity). Correspondingly, given a subregion $\mathcal{V} \subset \mathbb{R}^{4}$, the Lagrangian of the electromagnetic field in this subregion is given, as usual, by

$$
\begin{aligned}
& L_{e}\left(A_{0}, A_{1}, A_{2}, A_{3}\right):= \\
& \iiint \int_{\mathcal{V}}\left(\mathcal{L}_{e}\left(\left(A_{0}, A_{1}, A_{2}, A_{3}\right),\left\{K^{m n}\right\}_{m, n=0,1,2,3}\right)\left|\operatorname{det}\left(\left\{K^{m n}\right\}_{n, m=0,1,2,3}\right)\right|^{-\frac{1}{2}}\right) d x^{0} d x^{1} d x^{2} d x^{3} .
\end{aligned}
$$

In particular, the critical points of $L_{e}\left(A_{0}, A_{1}, A_{2}, A_{3}\right)$ must satisfy

$$
\frac{\delta L_{e}}{\delta A_{j}}\left(A_{0}, A_{1}, A_{2}, A_{3}\right)=0 \quad \forall j=0,1,2,3,
$$

and, thus by the Euler-Lagrange we deduce

$$
\begin{aligned}
\sum_{j=0}^{3} \frac{\partial}{\partial x^{j}}\left(\sum_{m=0}^{3} \sum_{n=0}^{3}\left|\operatorname{det}\left\{K^{p q}\right\}_{p, q=0,1,2,3}\right|^{-\frac{1}{2}} K^{k m} K^{j n}\left(\frac{\partial A_{n}}{\partial x^{m}}-\frac{\partial A_{m}}{\partial x^{n}}\right)\right) & \\
= & -4 \pi\left|\operatorname{det}\left\{K^{p q}\right\}_{p, q=0,1,2,3}\right|^{-\frac{1}{2}} j^{k} \quad \forall k=0,1,2,3 .
\end{aligned}
$$

\subsection{Correlated pseudo-metrics}

Definition 2.20. Consider two contravariant pseudo-metrics $\left\{J^{m n}\right\}_{m, n=0,1,2,3}$ and $\left\{K^{m n}\right\}_{m, n=0,1,2,3}$ on $\mathbb{R}^{4}$ and let $\left\{J_{m n}\right\}_{m, n=0,1,2,3}$ and $\left\{K_{m n}\right\}_{m, n=0,1,2,3}$ be the inverse covariant pseudo-metrics on $\mathbb{R}^{4}$, 
associated with $\left\{J^{m n}\right\}_{m, n=0,1,2,3}$ and $\left\{K^{m n}\right\}_{m, n=0,1,2,3}$, that satisfy

$$
\sum_{k=0}^{3} J^{m k} J_{k n}=\sum_{k=0}^{3} K^{m k} K_{k n}=\left\{\begin{array}{lll}
1 & \text { if } & m=n \\
0 & \text { if } & m \neq n
\end{array} \quad \forall m, n=0,1,2,3 .\right.
$$

Then, we say that $\left\{J^{m n}\right\}_{m, n=0,1,2,3}$ and $\left\{K^{m n}\right\}_{m, n=0,1,2,3}$ are two correlated pseudo-metrics, if there exist a four-covector of the time direction, $\left(w_{0}, w_{1}, w_{2}, w_{3}\right)$, weakly correlating with $\left\{J^{m n}\right\}_{m, n=0,1,2,3}$ and $\left\{K^{m n}\right\}_{m, n=0,1,2,3}$ simultaneously, so that we have

$$
\begin{array}{ll}
\sum_{j=0}^{3} \sum_{m=0}^{3} J^{j m} w_{j} w_{m}>0 & \forall\left(x^{0}, x^{1}, x^{2}, x^{3}\right) \in \mathbb{R}^{4}, \\
\sum_{j=0}^{3} \sum_{m=0}^{3} K^{j m} w_{j} w_{m}>0 & \forall\left(x^{0}, x^{1}, x^{2}, x^{3}\right) \in \mathbb{R}^{4},
\end{array}
$$

and moreover, there exists a four-covector field $\left(S_{0}, S_{1}, S_{2}, S_{3}\right)$, such that we have

$$
K_{j m}=\left(J_{j m}+w_{j} S_{m}+w_{m} S_{j}\right) \quad \forall 0 \leq j, m \leq 3 \quad \forall\left(x^{0}, x^{1}, x^{2}, x^{3}\right) \in \mathbb{R}^{4} .
$$

Theorem 2.5. Consider a contravariant pseudo-metric $\left\{J^{m n}\right\}_{m, n=0,1,2,3}$ on $\mathbb{R}^{4}$ and an arbitrary covariant four-covector field $\left(w_{0}, w_{1}, w_{2}, w_{3}\right)$, served as a time direction, and such that $\left\{J^{m n}\right\}_{m, n=0,1,2,3}$ and $\left(w_{0}, w_{1}, w_{2}, w_{3}\right)$ are weakly correlated. Furthermore, let $\left\{J_{m n}\right\}_{m, n=0,1,2,3}$ be the inverse covariant pseudo-metric on $\mathbb{R}^{4}$, associated with $\left\{J^{m n}\right\}_{m, n=0,1,2,3}$ and let $\left(r^{0}, r^{1}, r^{2}, r^{3}\right)$ be the potential of the generalized gravity, corresponding to $\left\{J^{m n}\right\}_{m, n=0,1,2,3}$ and $\left(w_{0}, w_{1}, w_{2}, w_{3}\right)$ and satisfying

$$
r^{m}:=\left(\sum_{j=0}^{3} \sum_{k=0}^{3} J^{j k} w_{j} w_{k}\right)^{-\frac{1}{2}}\left(\sum_{j=0}^{3} J^{m j} w_{j}\right) \quad \forall m=0,1,2,3 .
$$

Furthermore, as before, consider a contravariant tensor of three-dimensional Geometry $\left\{\Lambda^{m n}\right\}_{m, n=0,1,2,3}$, given by

$$
\Lambda^{j m}:=r^{j} r^{m}-J^{j m} \quad \forall j, m=0,1,2,3,
$$

Next, given an arbitrary contravariant four-vector field $\left(v^{0}, v^{1}, v^{2}, v^{3}\right)$, satisfying

$$
\sum_{j=0}^{3} v^{j} w_{j}>0
$$

define the contravariant symmetric tensor field $\left\{K^{m n}\right\}_{m, n=0,1,2,3}$ by the following relations:

$$
K^{j m}:=v^{j} v^{m}-\Lambda^{j m} \quad \forall j, m=0,1,2,3 .
$$

Then, $\left\{K^{m n}\right\}_{m, n=0,1,2,3}$ is a valid contravariant pseudo-metric. Moreover, the inverse to $\left\{K^{m n}\right\}_{m, n=0,1,2,3}$ covariant pseudo-metric $\left\{K_{m n}\right\}_{m, n=0,1,2,3}$ is given at every point in $\mathbb{R}^{4}$ by the following:

$$
K_{j m}=\left(J_{j m}+w_{j} S_{m}+w_{m} S_{j}\right) \quad \forall 0 \leq j, m \leq 3,
$$


where the covariant four-covector $\left(S_{0}, S_{1}, S_{2}, S_{3}\right)$ is defined by the following covariant relation:

$$
S_{m}:=\frac{1}{2}\left(\sum_{j=0}^{3} \sum_{k=0}^{3} J^{j k} w_{j} w_{k}\right)^{-\frac{1}{2}}\left(\sum_{j=0}^{3} K_{m j} r^{j}\right)-\frac{1}{2}\left(\sum_{j=0}^{3} v^{j} w_{j}\right)^{-1}\left(\sum_{j=0}^{3} J_{m j} v^{j}\right) \quad \forall 0 \leq m \leq 3 .
$$

Finally, the following covariant relations are valid

$$
\begin{gathered}
\sum_{j=0}^{3} \sum_{m=0}^{3} K_{j m} v^{j} v^{m}=1, \\
\sum_{j=0}^{3} K^{j m} w_{j}=\left(\sum_{j=0}^{3} v^{j} w_{j}\right) v^{m} \quad \forall m=0,1,2,3,
\end{gathered}
$$

and

$$
\sum_{m=0}^{3} \sum_{j=0}^{3} K^{j m} w_{j} w_{m}=\frac{\left(-\operatorname{det}\left(\left\{J_{m n}\right\}_{m, n=0,1,2,3}\right)\right)\left(\sum_{m=0}^{3} \sum_{n=0}^{3} J^{m n} w_{m} w_{n}\right)}{\left(-\operatorname{det}\left(\left\{K_{m n}\right\}_{m, n=0,1,2,3}\right)\right)}=\left(\sum_{j=0}^{3} v^{j} w_{j}\right)^{2} .
$$

So, $\left\{J^{m n}\right\}_{m, n=0,1,2,3}$ and $\left\{K^{m n}\right\}_{m, n=0,1,2,3}$ are two correlated pseudo-metrics (see Definition 2.20). Moreover, $K^{m n}$ and $\left(w_{0}, w_{1}, w_{2}, w_{3}\right)$ are weakly correlated and $\left(v^{0}, v^{1}, v^{2}, v^{3}\right)$ is a potential of generalized gravity, corresponding to the couple $K^{m n}$ and $\left(w_{0}, w_{1}, w_{2}, w_{3}\right)$.

Theorem 2.6. Consider a contravariant pseudo-metric $\left\{J^{m n}\right\}_{m, n=0,1,2,3}$ on $\mathbb{R}^{4}$ and an arbitrary covariant four-covector field $\left(w_{0}, w_{1}, w_{2}, w_{3}\right)$, served as a time direction, such that $\left\{J^{m n}\right\}_{m, n=0,1,2,3}$ and $\left(w_{0}, w_{1}, w_{2}, w_{3}\right)$ are weakly correlated. Furthermore, let $\left\{J_{m n}\right\}_{m, n=0,1,2,3}$ be the inverse covariant pseudo-metric on $\mathbb{R}^{4}$, associated with $\left\{J^{m n}\right\}_{m, n=0,1,2,3}$ and let $\left(r^{0}, r^{1}, r^{2}, r^{3}\right)$ be the potential of the generalized gravity, corresponding to $\left\{J^{m n}\right\}_{m, n=0,1,2,3}$ and $\left(w_{0}, w_{1}, w_{2}, w_{3}\right)$ and satisfying (2.120). Furthermore, as before, consider a contravariant tensor of three-dimensional Geometry $\left\{\Lambda^{m n}\right\}_{m, n=0,1,2,3}$, given by (2.121). Next consider, an arbitrary covariant four-covector $\left(S_{0}, S_{1}, S_{2}, S_{3}\right)$ such that a two-times covariant tensor field $\left\{K_{m n}\right\}_{m, n=0,1,2,3}$, defined by

$$
K_{j m}=\left(J_{j m}+w_{j} S_{m}+w_{m} S_{j}\right) \quad \forall 0 \leq j, m \leq 3,
$$

satisfies the following at every point in $\mathbb{R}^{4}$ :

$$
\operatorname{det}\left(\left\{K_{m n}\right\}_{m, n=0,1,2,3}\right)<0 .
$$

Then, the inverse to $\left\{K_{m n}\right\}_{m, n=0,1,2,3}$ two-times contravariant symmetric tensor field $\left\{K^{m n}\right\}_{m, n=0,1,2,3}$ is given by

$$
K^{m n}=v^{m} v^{n}-\Lambda_{m n} \quad \forall m, n=0,1,2,3 .
$$

where the contravariant vector field $\left(v^{0}, v^{1}, v^{2}, v^{3}\right)$ is defined by the following covariant relation:

$$
\begin{array}{r}
v^{m}:=\left(\sqrt{\frac{-\operatorname{det}\left(\left\{J_{k n}\right\}_{k, n=0,1,2,3}\right)}{-\operatorname{det}\left(\left\{K_{k n}\right\}_{k, n=0,1,2,3}\right)}}\right)\left(\left(\sqrt{\sum_{j=0}^{3} \sum_{k=0}^{3} J^{j k} w_{j} w_{k}}\right)\left(\sum_{j=0}^{3} \Lambda^{m j} S_{j}\right)+r^{m}\right) \\
\forall m=0,1,2,3 .
\end{array}
$$


Furthermore, we also have

$$
\left(\sum_{j=0}^{3} v^{j} w_{j}\right)>0
$$

and $\left\{K^{m n}\right\}_{m, n=0,1,2,3}$ is a valid contravariant pseudo-metric. Moreover, the covariant relations in (2.125), (2.126), (2.127) and (2.128) are also valid. So, $\left\{J^{m n}\right\}_{m, n=0,1,2,3}$ and $\left\{K^{m n}\right\}_{m, n=0,1,2,3}$ are two correlated pseudo-metrics. Moreover, $K^{m n}$ and $\left(w_{0}, w_{1}, w_{2}, w_{3}\right)$ are weakly correlated and $\left(v^{0}, v^{1}, v^{2}, v^{3}\right)$ is a potential of generalized gravity, corresponding to $K^{m n}$ and $\left(w_{0}, w_{1}, w_{2}, w_{3}\right)$.

Corollary 2.10. Consider a contravariant pseudo-metric $\left\{J^{m n}\right\}_{m, n=0,1,2,3}$ on $\mathbb{R}^{4}$ and an arbitrary scalar field $\varphi: \mathbb{R}^{4} \rightarrow \mathbb{R}$, served as a global time, such that

$$
\sum_{j=0}^{3} \sum_{m=0}^{3} J^{j m} \frac{\partial \varphi}{\partial x^{j}} \frac{\partial \varphi}{\partial x^{m}}=1 \quad \forall\left(x^{0}, x^{1}, x^{2}, x^{3}\right) \in \mathbb{R}^{4} .
$$

Furthermore, let $\left\{J_{m n}\right\}_{m, n=0,1,2,3}$ be the inverse covariant pseudo-metric on $\mathbb{R}^{4}$, associated with $\left\{J^{m n}\right\}_{m, n=0,1,2,3}$ and let $\left(r^{0}, r^{1}, r^{2}, r^{3}\right)$ be the contravariant four-vector field, defined by

$$
r^{m}:=\sum_{j=0}^{3} J^{m j} \frac{\partial \varphi}{\partial x^{j}} \quad \forall m=0,1,2,3 .
$$

Furthermore, as before, consider a contravariant tensor of three-dimensional Geometry $\left\{\Lambda^{m n}\right\}_{m, n=0,1,2,3}$, given by

$$
\Lambda^{j m}:=r^{j} r^{m}-J^{j m} \quad \forall j, m=0,1,2,3 .
$$

Next, given an arbitrary contravariant four-vector field $\left(v^{0}, v^{1}, v^{2}, v^{3}\right)$, satisfying

$$
\sum_{j=0}^{3} v^{j} \frac{\partial \varphi}{\partial x^{j}}=1
$$

define the contravariant symmetric tensor field $\left\{K^{m n}\right\}_{m, n=0,1,2,3}$ by the following relations:

$$
K^{j m}:=v^{j} v^{m}-\Lambda^{j m} \quad \forall j, m=0,1,2,3 .
$$

Then, $\left\{K^{m n}\right\}_{m, n=0,1,2,3}$ is a valid contravariant pseudo-metric and we have

$$
\sum_{m=0}^{3} \sum_{j=0}^{3} K^{j m} \frac{\partial \varphi}{\partial x^{j}} \frac{\partial \varphi}{\partial x^{m}}=1
$$

Moreover, the inverse to $\left\{K^{m n}\right\}_{m, n=0,1,2,3}$ covariant pseudo-metric $\left\{K_{m n}\right\}_{m, n=0,1,2,3}$ is given at every point in $\mathbb{R}^{4}$ by the following:

$$
K_{j m}=\left(J_{j m}+S_{m} \frac{\partial \varphi}{\partial x^{j}}+S_{j} \frac{\partial \varphi}{\partial x^{m}}\right) \quad \forall 0 \leq j, m \leq 3,
$$

where the covariant four-covector $\left(S_{0}, S_{1}, S_{2}, S_{3}\right)$ is defined by the following covariant relation:

$$
S_{m}:=\sum_{j=0}^{3} \frac{1}{2} K_{m j} r^{j}-\sum_{j=0}^{3} \frac{1}{2} J_{m j} v^{j} \quad \forall 0 \leq m \leq 3 .
$$


Finally, the following covariant relations are valid

$$
\begin{gathered}
\sum_{j=0}^{3} \sum_{m=0}^{3} K_{j m} v^{j} v^{m}=1, \\
\sum_{j=0}^{3} K^{j m} \frac{\partial \varphi}{\partial x^{j}}=v^{m} \quad \forall m=0,1,2,3, \\
\operatorname{det}\left(\left\{J_{m n}\right\}_{m, n=0,1,2,3}\right)=\operatorname{det}\left(\left\{K_{m n}\right\}_{m, n=0,1,2,3}\right) .
\end{gathered}
$$

So, $\left\{J^{m n}\right\}_{m, n=0,1,2,3}$ and $\left\{K^{m n}\right\}_{m, n=0,1,2,3}$ are two correlated pseudo-metrics, that both correlated with the same global time $\varphi$ and $\left(v^{0}, v^{1}, v^{2}, v^{3}\right)$ is a potential of generalized gravity, corresponding to $K^{m n}$ and $\varphi$.

Corollary 2.11. Consider a contravariant pseudo-metric $\left\{J^{m n}\right\}_{m, n=0,1,2,3}$ on $\mathbb{R}^{4}$ and an arbitrary scalar field $\varphi: \mathbb{R}^{4} \rightarrow \mathbb{R}$, served as a global time, such that we have (2.134). Next, let $\left\{J_{m n}\right\}_{m, n=0,1,2,3}$ be the inverse covariant pseudo-metric on $\mathbb{R}^{4}$, associated with $\left\{J^{m n}\right\}_{m, n=0,1,2,3}$ and let $\left(r^{0}, r^{1}, r^{2}, r^{3}\right)$ be the contravariant four-vector field, defined by (2.135). Furthermore, as before, consider a contravariant tensor of three-dimensional Geometry $\left\{\Lambda^{m n}\right\}_{m, n=0,1,2,3}$, given by (2.136). Next consider, an arbitrary covariant four-covector $\left(S_{0}, S_{1}, S_{2}, S_{3}\right)$ such that a two-times covariant tensor field $\left\{K^{m n}\right\}_{m, n=0,1,2,3}$, defined by

$$
K_{j m}=\left(J_{j m}+S_{m} \frac{\partial \varphi}{\partial x^{j}}+S_{j} \frac{\partial \varphi}{\partial x^{m}}\right) \quad \forall 0 \leq j, m \leq 3,
$$

satisfies the following covariant relation at every point in $\mathbb{R}^{4}$ :

$$
\operatorname{det}\left(\left\{K_{m n}\right\}_{m, n=0,1,2,3}\right)=\operatorname{det}\left(\left\{J_{m n}\right\}_{m, n=0,1,2,3}\right) .
$$

Then, the inverse to $\left\{K^{m n}\right\}_{m, n=0,1,2,3}$ two-times contravariant symmetric tensor field $\left\{K_{m n}\right\}_{m, n=0,1,2,3}$ is given by

$$
K^{m n}=v^{m} v^{n}-\Lambda_{m n} \quad \forall m, n=0,1,2,3 .
$$

where the contravariant vector field $\left(v^{0}, v^{1}, v^{2}, v^{3}\right)$ is defined by the following covariant relation:

$$
v^{m}:=\left(\sum_{j=0}^{3} \Lambda^{m j} S_{j}+r^{m}\right) \quad \forall m=0,1,2,3 .
$$

Moreover, we also have

$$
\sum_{j=0}^{3} v^{j} \frac{\partial \varphi}{\partial x^{j}}=1
$$

$\left\{K^{m n}\right\}_{m, n=0,1,2,3}$ is a valid contravariant pseudo-metric, and

$$
\sum_{m=0}^{3} \sum_{j=0}^{3} K^{j m} \frac{\partial \varphi}{\partial x^{j}} \frac{\partial \varphi}{\partial x^{m}}=1
$$

Moreover, the covariant relations in (2.141), (2.142) and (2.143) are also valid. So, $\left\{J^{m n}\right\}_{m, n=0,1,2,3}$ and $\left\{K^{m n}\right\}_{m, n=0,1,2,3}$ are two correlated pseudo-metrics, that both correlated with the same global time $\varphi$ and $\left(v^{0}, v^{1}, v^{2}, v^{3}\right)$ is a potential of generalized gravity, corresponding to $K^{m n}$ and $\varphi$. 


\subsection{Kinematically correlated models of the genuine gravity}

Definition 2.21. Consider the strongly-correlated kinematical contravariant pseudo-metric $\left\{J^{m n}\right\}_{m, n=0,1,2,3}$ and the kinematical global time $\varphi$, forming a standard kinematical Lorentz's structure with global time on $\mathbb{R}^{4}$, as in Proposition 2.7 and Remark 2.6. Furthermore, let $\left\{J_{m n}\right\}_{m, n=0,1,2,3}$ be the inverse covariant pseudo-metric on $\mathbb{R}^{4}$, associated with $\left\{J^{m n}\right\}_{m, n=0,1,2,3}$. Given, an arbitrary dynamical four-covector of the time direction, $\left(w_{0}, w_{1}, w_{2}, w_{3}\right)$ (formally unrelated to the kinematical global time $\varphi$ ), which is weakly correlated with $\left\{J^{m n}\right\}_{m, n=0,1,2,3}$ and an arbitrary four-covector field $\left(S_{0}, S_{1}, S_{2}, S_{3}\right)$, which we call the four-covector of genuine gravity, consider the two-times covariant tensor $\left\{K_{m n}\right\}_{m, n=0,1,2,3}$ defined by:

$$
K_{j m}=\left(J_{j m}+w_{j} S_{m}+w_{m} S_{j}\right) \quad \forall 0 \leq j, m \leq 3 \quad \forall\left(x^{0}, x^{1}, x^{2}, x^{3}\right) \in \mathbb{R}^{4},
$$

and assume that $\left(S_{0}, S_{1}, S_{2}, S_{3}\right)$ is such that $\left\{K_{m n}\right\}_{m, n=0,1,2,3}$ in (2.151) satisfies

$$
\operatorname{det}\left(\left\{K_{m n}\right\}_{m, n=0,1,2,3}\right)=\operatorname{det}\left(\left\{J_{m n}\right\}_{m, n=0,1,2,3}\right) \quad \forall\left(x^{0}, x^{1}, x^{2}, x^{3}\right) \in \mathbb{R}^{4}
$$

( the last equality is obviously independent on the choice of coordinate system). Then, by Theorem 2.6 the inverse to $\left\{K_{m n}\right\}_{m, n=0,1,2,3}$ two-times contravariant tensor $\left\{K^{m n}\right\}_{m, n=0,1,2,3}$ is a valid contravariant pseudometric, weakly correlated with the time-direction $\left(w_{0}, w_{1}, w_{2}, w_{3}\right)$. Moreover, we call such a pseudometric $\left\{K^{m n}\right\}_{m, n=0,1,2,3}$, with time-direction $\left(w_{0}, w_{1}, w_{2}, w_{3}\right)$, kinematically correlated pseudometric with time-direction $\left(w_{0}, w_{1}, w_{2}, w_{3}\right)$, corresponding to the covector of genuine gravity $\left(S_{0}, S_{1}, S_{2}, S_{3}\right)$.

Remark 2.8. If $\left\{K^{m n}\right\}_{m, n=0,1,2,3}$, with time-direction $\left(w_{0}, w_{1}, w_{2}, w_{3}\right)$, is a kinematically $\underline{\text { correlated }}$ pseudometric with time-direction $\left(w_{0}, w_{1}, w_{2}, w_{3}\right)$, corresponding to the covector of genuine gravity $\left(S_{0}, S_{1}, S_{2}, S_{3}\right)$, then by Theorem $2.6\left\{J^{m n}\right\}_{m, n=0,1,2,3}$ and $\left\{K^{m n}\right\}_{m, n=0,1,2,3}$ are two correlated pseudo-metrics (see Definition 2.20). Moreover, denoting by $\left(r^{0}, r^{1}, r^{2}, r^{3}\right)$ the contravariant fourvector of the generalized gravity, corresponding to $\left\{J^{m n}\right\}_{m, n=0,1,2,3}$ and $\left(w_{0}, w_{1}, w_{2}, w_{3}\right)$, which satisfies

$$
r^{m}:=\left(\sum_{j=0}^{3} \sum_{k=0}^{3} J^{j k} w_{j} w_{k}\right)^{-\frac{1}{2}}\left(\sum_{j=0}^{3} J^{m j} w_{j}\right) \quad \forall m=0,1,2,3,
$$

and considering a contravariant tensor of three-dimensional Geometry $\left\{\Lambda^{m n}\right\}_{m, n=0,1,2,3}$, given by

$$
\Lambda^{m n}:=r^{m} r^{n}-J^{m n} \quad \forall m, n=0,1,2,3,
$$

using Theorem 2.6, we have

$$
K^{m n}=v^{m} v^{n}-\Lambda_{m n}=J^{m n}+v^{m} v^{n}-r^{m} r^{n} \quad \forall m, n=0,1,2,3 .
$$

where the contravariant vector of generalized gravity $\left(v^{0}, v^{1}, v^{2}, v^{3}\right)$ is defined by the following covariant relation:

$$
v^{m}:=\left(\sqrt{\sum_{j=0}^{3} \sum_{k=0}^{3} J^{j k} w_{j} w_{k}}\right)\left(\sum_{j=0}^{3} \Lambda^{m j} S_{j}\right)+r^{m} \quad \forall m=0,1,2,3,
$$


and both couples $\left\{J^{m n}\right\}_{m, n=0,1,2,3},\left(w_{0}, w_{1}, w_{2}, w_{3}\right)$, and $\left\{K^{m n}\right\}_{m, n=0,1,2,3},\left(w_{0}, w_{1}, w_{2}, w_{3}\right)$ are weakly correlated. Moreover, we also have

$$
\left(\sum_{j=0}^{3} v^{j} w_{j}\right)>0
$$

and the following covariant relations are valid

$$
\begin{gathered}
S_{m}:=\frac{1}{2}\left(\sum_{j=0}^{3} \sum_{k=0}^{3} J^{j k} w_{j} w_{k}\right)^{-\frac{1}{2}}\left(\sum_{j=0}^{3} K_{m j} r^{j}\right)-\frac{1}{2}\left(\sum_{j=0}^{3} v^{j} w_{j}\right)^{-1}\left(\sum_{j=0}^{3} J_{m j} v^{j}\right) \quad \forall 0 \leq m \leq 3, \\
\sum_{j=0}^{3} \sum_{m=0}^{3} K_{j m} v^{j} v^{m}=1 \\
\sum_{j=0}^{3} K^{j m} w_{j}=\left(\sum_{j=0}^{3} v^{j} w_{j}\right) v^{m} \quad \forall m=0,1,2,3
\end{gathered}
$$

and

$$
\sum_{m=0}^{3} \sum_{j=0}^{3} K^{j m} w_{j} w_{m}=\sum_{m=0}^{3} \sum_{n=0}^{3} J^{m n} w_{m} w_{n}=\left(\sum_{j=0}^{3} v^{j} w_{j}\right)^{2}>0 .
$$

Note, however, that the dynamical time-direction $\left(w_{0}, w_{1}, w_{2}, w_{3}\right)$ can differ from kinematical timedirection $\left(\frac{\partial \varphi}{\partial x^{0}}, \frac{\partial \varphi}{\partial x^{1}}, \frac{\partial \varphi}{\partial x^{2}}, \frac{\partial \varphi}{\partial x^{3}}\right)$ and the dynamical tensor of three-dimensional Geometry $\left\{\Lambda^{m n}\right\}_{m, n=0,1,2,3}$ can differ from the kinematical tensor of three-dimensional Geometry $\left\{\Theta^{m n}\right\}_{m, n=0,1,2,3}$, given by

$$
\Theta^{m n}:=\left(\sum_{j=0}^{3} J^{m j} \frac{\partial \varphi}{\partial x^{j}}\right)\left(\sum_{j=0}^{3} J^{n j} \frac{\partial \varphi}{\partial x^{j}}\right)-J^{m n} \quad \forall m, n=0,1,2,3 .
$$

Finally, note that if the covector of genuine gravity $\left(S_{0}, S_{1}, S_{2}, S_{3}\right)$ vanishes at some point, then by (2.151) at this point we have

$$
K_{j m}=J_{j m} \quad \forall 0 \leq j, m \leq 3,
$$

correspondingly to the First Law of Newton (Axiom I).

Definition 2.22. Consider the strongly-correlated kinematical contravariant pseudo-metric $\left\{J^{m n}\right\}_{m, n=0,1,2,3}$ and the kinematical global time $\varphi$, forming a standard kinematical Lorentz's structure with global time on $\mathbb{R}^{4}$, as in Proposition 2.7 and Remark 2.6. Furthermore, let $\left\{J_{m n}\right\}_{m, n=0,1,2,3}$ be the inverse covariant pseudo-metric on $\mathbb{R}^{4}$, associated with $\left\{J^{m n}\right\}_{m, n=0,1,2,3}$. Then given a four-covector field $\left(S_{0}, S_{1}, S_{2}, S_{3}\right)$, which we call the four-covector of genuine gravity, consider the two-times covariant tensor $\left\{\hat{K}_{m n}\right\}_{m, n=0,1,2,3}$ defined by:

$$
\hat{K}_{j m}=\left(J_{j m}+S_{m} \frac{\partial \varphi}{\partial x^{j}}+\frac{\partial \varphi}{\partial x^{m}} S_{j}\right) \quad \forall 0 \leq j, m \leq 3 \quad \forall\left(x^{0}, x^{1}, x^{2}, x^{3}\right) \in \mathbb{R}^{4},
$$

and assume that $\left(S_{0}, S_{1}, S_{2}, S_{3}\right)$ is such that $\left\{\hat{K}_{m n}\right\}_{m, n=0,1,2,3}$ in (2.164) satisfies

$$
\operatorname{det}\left(\left\{\hat{K}_{m n}\right\}_{m, n=0,1,2,3}\right)=\operatorname{det}\left(\left\{J_{m n}\right\}_{m, n=0,1,2,3}\right) \quad \forall\left(x^{0}, x^{1}, x^{2}, x^{3}\right) \in \mathbb{R}^{4} .
$$


Then, as before, the inverse to $\left\{\hat{K}_{m n}\right\}_{m, n=0,1,2,3}$ two-times contravariant tensor $\left\{\hat{K}^{m n}\right\}_{m, n=0,1,2,3}$ is a valid contravariant pseudometric, strongly correlated with the kinematical global time $\varphi$. Moreover, we call such a pseudometric $\left\{\hat{K}^{m n}\right\}_{m, n=0,1,2,3}$, with global time $\varphi$, kinematically $\underline{\text { semi-scalar }} \underline{\text { super- }}$ correlated pseudometric with kinematical global time $\varphi$, corresponding to the covector of genuine gravity $\left(S_{0}, S_{1}, S_{2}, S_{3}\right)$.

Remark 2.9. If $\left\{K^{m n}\right\}_{m, n=0,1,2,3}$, with kinematical global time $\varphi$, is a kinematically semi-scalar super-correlated pseudometric with kinematical global time $\varphi$, corresponding to the covector of genuine gravity $\left(S_{0}, S_{1}, S_{2}, S_{3}\right)$, then, denoting by $\left(r^{0}, r^{1}, r^{2}, r^{3}\right)$ the contravariant four-vector of the inertia, corresponding to $\left\{J^{m n}\right\}_{m, n=0,1,2,3}$ and $\varphi$, which satisfies

$$
r^{m}:=\sum_{j=0}^{3} J^{m j} \frac{\partial \varphi}{\partial x^{j}} \quad \forall m=0,1,2,3,
$$

and considering a contravariant tensor of three-dimensional Geometry $\left\{\Lambda^{m n}\right\}_{m, n=0,1,2,3}$, given by

$$
\Lambda^{m n}:=r^{m} r^{n}-J^{m n}=\left(\sum_{j=0}^{3} J^{m j} \frac{\partial \varphi}{\partial x^{j}}\right)\left(\sum_{j=0}^{3} J^{n j} \frac{\partial \varphi}{\partial x^{j}}\right)-J^{m n} \quad \forall m, n=0,1,2,3,
$$

we have

$$
K^{m n}=v^{m} v^{n}-\Lambda_{m n}=J^{m n}+v^{m} v^{n}-r^{m} r^{n} \quad \forall m, n=0,1,2,3 .
$$

where the contravariant vector of generalized gravity $\left(v^{0}, v^{1}, v^{2}, v^{3}\right)$ is defined by the following covariant relation:

$$
v^{m}:=\left(\sum_{j=0}^{3} \Lambda^{m j} S_{j}\right)+r^{m} \quad \forall m=0,1,2,3,
$$

and both couples $\left\{J^{m n}\right\}_{m, n=0,1,2,3}, \varphi$, and $\left\{K^{m n}\right\}_{m, n=0,1,2,3}, \varphi$ are strongly correlated. Moreover, the following covariant relations are valid

$$
\begin{gathered}
\left(\sum_{j=0}^{3} v^{j} \frac{\partial \varphi}{\partial x^{j}}\right)=1, \\
S_{m}:=\frac{1}{2}\left(\sum_{j=0}^{3} K_{m j} r^{j}\right)-\frac{1}{2}\left(\sum_{j=0}^{3} J_{m j} v^{j}\right) \quad \forall 0 \leq m \leq 3, \\
\sum_{j=0}^{3} \sum_{m=0}^{3} K_{j m} v^{j} v^{m}=1, \\
\sum_{j=0}^{3} \sum_{m=0}^{3} \frac{\partial \varphi}{\partial x^{j}}=v^{m} \quad \forall m=0,1,2,3, \\
\sum^{j m} \frac{\partial \varphi}{\partial x^{j}} \frac{\partial \varphi}{\partial x^{m}}=1,
\end{gathered}
$$

and

$$
\sum_{j=0}^{3} \sum_{m=0}^{3} J^{j m} \frac{\partial \varphi}{\partial x^{j}} \frac{\partial \varphi}{\partial x^{m}}=1
$$


Finally, note that the dynamical global time equals to kinematical time $\varphi$ and the dynamical tensor of three-dimensional Geometry $\left\{\Lambda^{m n}\right\}_{m, n=0,1,2,3}$ equals to the kinematical tensor of three-dimensional Geometry $\left\{\Theta^{m n}\right\}_{m, n=0,1,2,3}$, so that

$$
\Lambda^{m n}=\Theta^{m n}:=\left(\sum_{j=0}^{3} J^{m j} \frac{\partial \varphi}{\partial x^{j}}\right)\left(\sum_{j=0}^{3} J^{n j} \frac{\partial \varphi}{\partial x^{j}}\right)-J^{m n} \quad \forall m, n=0,1,2,3 .
$$

\subsection{Lagrangian for dynamical time-direction and its limiting case}

Consider the strongly-correlated kinematical covariant pseudo-metric $\left\{J^{m n}\right\}_{m, n=0,1,2,3}$ and the kinematical global time $\varphi$, forming a standard kinematical Lorentz's structure with global time on $\mathbb{R}^{4}$. Furthermore, consider an arbitrary four-covector of the time-direction $\left(w_{0}, w_{1}, w_{2}, w_{3}\right)$. Next, consider a Lagrangian density $\mathcal{L}_{R}:=\mathcal{L}_{R}\left(\left(w_{0}, w_{1}, w_{2}, w_{3}\right),\left(x^{0}, x^{1}, x^{2}, x^{3}\right)\right)$, defined by

$$
\begin{aligned}
\mathcal{L}_{R}\left(\left(w_{0}, w_{1}, w_{2}, w_{3}\right),\left(x^{0}, x^{1}, x^{2}, x^{3}\right)\right):= \\
-\lambda \alpha \mu\left(\sum_{n=0}^{3} \sum_{k=0}^{3} \sum_{m=0}^{3} \sum_{p=0}^{3} \frac{1}{4} J^{m n} J^{p k}\left(\frac{\partial w_{p}}{\partial x^{m}}-\frac{\partial w_{m}}{\partial x^{p}}\right)\left(\frac{\partial w_{k}}{\partial x^{n}}-\frac{\partial w_{n}}{\partial x^{k}}\right)\right) \\
\quad-\frac{\alpha \nu}{4}\left(\sum_{m=0}^{3} \sum_{n=0}^{3} J^{m n} w_{m} w_{n}-1\right)^{2}-G\left(\left(w_{0}, w_{1}, w_{2}, w_{3}\right),\left(x^{0}, x^{1}, x^{2}, x^{3}\right)\right),
\end{aligned}
$$

where $\alpha \neq 0, \lambda \neq 0$ are real dimensionless constants, such that

$$
|\alpha| \gg 1 \quad \text { and } \quad|\lambda| \gg 1 .
$$

and $\mu \neq 0, \nu \neq 0$ are real constants. Here $G$ is some given function and, for simplicity of the notation, we omit the dependence of the function $G$ by the additional physical fields (like dependence on dynamical pseudo-metric $\left\{K^{m n}\right\}_{m, n=0,1,2,3}$, mass densities, electromagnetic fields et.al) and express it throughout explicit dependence on $\left(x^{0}, x^{1}, x^{2}, x^{3}\right)$. Next, given a subregion $\mathcal{V} \subset \mathbb{R}^{4}$, we define the Lagrangian on the region $\mathcal{V}$ as:

$$
\begin{aligned}
& L_{R}\left(\left(w_{0}, w_{1}, w_{2}, w_{3}\right)\right):= \\
& \quad \iiint \int_{\mathcal{V}}\left(\mathcal{L}_{R}\left(\left(w_{0}, w_{1}, w_{2}, w_{3}\right),\left(x^{0}, x^{1}, x^{2}, x^{3}\right)\right)\left|\operatorname{det}\left(\left\{J^{m n}\right\}_{n, m=0,1,2,3}\right)\right|^{-\frac{1}{2}}\right) d x^{0} d x^{1} d x^{2} d x^{3} .
\end{aligned}
$$

Note that det $\left(\left\{J^{m n}\right\}_{n, m=0,1,2,3}\right)$ is independent on $\left(w_{0}, w_{1}, w_{2}, w_{3}\right)$. In particular, the critical points of $L_{N}$ must satisfy

$$
\frac{\delta L_{R}}{\delta w_{j}}\left(\left(w_{0}, w_{1}, w_{2}, w_{3}\right)\right)=0 \quad \forall j=0,1,2,3,
$$


and, since $\operatorname{det}\left(\left\{J^{m n}\right\}_{n, m=0,1,2,3}\right)$ is independent on $\left(w_{0}, w_{1}, w_{2}, w_{3}\right)$, by the Euler-Lagrange we deduce

$$
\begin{array}{r}
\lambda \alpha \mu\left(\sum_{n=0}^{3} \sum_{k=0}^{3} \sum_{m=0}^{3} \frac{\partial}{\partial x^{m}}\left\{J^{m n} J^{j k}\left(\frac{\partial w_{k}}{\partial x^{n}}-\frac{\partial w_{n}}{\partial x^{k}}\right)\right\}\right) \\
-\alpha \nu\left(\sum_{m=0}^{3} \sum_{n=0}^{3} J^{m n} w_{m} w_{n}-1\right)\left(\sum_{n=0}^{3} J^{j n} w_{n}\right)-\frac{\partial G}{\partial w_{j}}\left(\left(w_{0}, w_{1}, w_{2}, w_{3}\right),\left(x^{0}, x^{1}, x^{2}, x^{3}\right)\right)=0 \\
\forall j=0,1,2,3 .
\end{array}
$$

So we have

$$
\begin{array}{r}
\mu\left(\sum_{n=0}^{3} \sum_{k=0}^{3} \sum_{m=0}^{3} \frac{\partial}{\partial x^{m}}\left\{J^{m n} J^{j k}\left(\frac{\partial w_{k}}{\partial x^{n}}-\frac{\partial w_{n}}{\partial x^{k}}\right)\right\}\right) \\
-\frac{\nu}{\lambda}\left(\sum_{m=0}^{3} \sum_{n=0}^{3} J^{m n} w_{m} w_{n}-1\right)\left(\sum_{n=0}^{3} J^{j n} w_{n}\right)-\frac{1}{\alpha \lambda} \frac{\partial G}{\partial w_{j}}\left(\left(w_{0}, w_{1}, w_{2}, w_{3}\right),\left(x^{0}, x^{1}, x^{2}, x^{3}\right)\right)=0 \\
\forall j=0,1,2,3 .
\end{array}
$$

In particular, in the case of (2.178), taking

$$
|\alpha| \rightarrow+\infty
$$

we approximate (2.182) as:

$$
\begin{aligned}
\mu\left(\sum_{n=0}^{3} \sum_{k=0}^{3} \sum_{m=0}^{3} \frac{\partial}{\partial x^{m}}\right. & \left.\left\{J^{m n} J^{j k}\left(\frac{\partial w_{k}}{\partial x^{n}}-\frac{\partial w_{n}}{\partial x^{k}}\right)\right\}\right) \\
- & \frac{\nu}{\lambda}\left(\sum_{m=0}^{3} \sum_{n=0}^{3} J^{m n} w_{m} w_{n}-1\right)\left(\sum_{n=0}^{3} J^{j n} w_{n}\right)=0 \quad \forall j=0,1,2,3 .
\end{aligned}
$$

In particular, by (2.184) we obtain:

$$
\begin{aligned}
\sum_{j=0}^{3} \frac{\partial}{\partial x^{j}}\left\{\left(\sum_{m=0}^{3} \sum_{n=0}^{3} J^{m n} w_{m} w_{n}-1\right)\left(\sum_{n=0}^{3} J^{j n} w_{n}\right)\right\}= \\
\sum_{j=0}^{3} \frac{\lambda \mu}{\nu} \frac{\partial}{\partial x^{j}}\left(\sum_{n=0}^{3} \sum_{k=0}^{3} \sum_{m=0}^{3} \frac{\partial}{\partial x^{m}}\left\{J^{m n} J^{j k}\left(\frac{\partial w_{k}}{\partial x^{n}}-\frac{\partial w_{n}}{\partial x^{k}}\right)\right\}\right)=0 .
\end{aligned}
$$

Moreover, taking

$$
|\lambda| \rightarrow+\infty
$$

we approximate (2.184) as:

$$
\left(\sum_{n=0}^{3} \sum_{k=0}^{3} \sum_{m=0}^{3} \frac{\partial}{\partial x^{m}}\left\{J^{m n} J^{j k}\left(\frac{\partial w_{k}}{\partial x^{n}}-\frac{\partial w_{n}}{\partial x^{k}}\right)\right\}\right) \rightarrow 0 \quad \forall j=0,1,2,3 .
$$

In particular, the last equation

$$
\left(\sum_{n=0}^{3} \sum_{k=0}^{3} \sum_{m=0}^{3} \frac{\partial}{\partial x^{m}}\left\{J^{m n} J^{j k}\left(\frac{\partial w_{k}}{\partial x^{n}}-\frac{\partial w_{n}}{\partial x^{k}}\right)\right\}\right)=0 \quad \forall j=0,1,2,3,
$$


is the same as the classical Maxwell equations in vacuum with vanishing charges and currents, where the four-covector of the electromagnetic potential $\left(A_{0}, A_{1}, A_{2}, A_{3}\right)$ is replaced by the four-covector $\left(w_{0}, w_{1}, w_{2}, w_{3}\right)$. Thus, ignoring wave-type solutions of (2.188), that assumed to be negligible, we deduce by (2.188) that there exist a proper scalar $\psi$, such that we have

$$
\left(w_{0}, w_{1}, w_{2}, w_{3}\right)=\left(\frac{\partial \psi}{\partial x^{0}}, \frac{\partial \psi}{\partial x^{1}}, \frac{\partial \psi}{\partial x^{2}}, \frac{\partial \psi}{\partial x^{3}}\right) \quad \forall\left(x^{0}, x^{1}, x^{2}, x^{3}\right) \in \mathbb{R}^{4} .
$$

Thus, inserting (2.189) into (2.185) gives,

$$
\sum_{j=0}^{3} \frac{\partial}{\partial x^{j}}\left\{\left(\sum_{m=0}^{3} \sum_{n=0}^{3} J^{m n} \frac{\partial \psi}{\partial x^{m}} \frac{\partial \psi}{\partial x^{n}}-1\right)\left(\sum_{n=0}^{3} J^{j n} \frac{\partial \psi}{\partial x^{n}}\right)\right\}=0 .
$$

Therefore, using (2.190), with the help of Lemma 11.1 from the Appendix, we deduce the following eikonal-type equation

$$
\sum_{m=0}^{3} \sum_{n=0}^{3} J^{m n} \frac{\partial \psi}{\partial x^{m}} \frac{\partial \psi}{\partial x^{n}}=1 \quad \forall\left(x^{0}, x^{1}, x^{2}, x^{3}\right) \in \mathbb{R}^{4} .
$$

So the global time $\psi$ is correlated with pseudo-metric $\left\{J^{m n}\right\}_{m, n=0,1,2,3}$. On the other hand, for the kinematical global time $\varphi$ we also have

$$
\sum_{m=0}^{3} \sum_{n=0}^{3} J^{m n} \frac{\partial \varphi}{\partial x^{m}} \frac{\partial \varphi}{\partial x^{n}}=1 \quad \forall\left(x^{0}, x^{1}, x^{2}, x^{3}\right) \in \mathbb{R}^{4} .
$$

Therefore, in the case, where $\psi$ and $\varphi$ coincide in some initial surface, by (2.191) and (2.192) we deduce that, in the limiting case (2.183) we have for the dynamical time direction, that

$$
\left(w_{0}, w_{1}, w_{2}, w_{3}\right)=\left(\frac{\partial \varphi}{\partial x^{0}}, \frac{\partial \varphi}{\partial x^{1}}, \frac{\partial \varphi}{\partial x^{2}}, \frac{\partial \varphi}{\partial x^{3}}\right) \quad \forall\left(x^{0}, x^{1}, x^{2}, x^{3}\right) \in \mathbb{R}^{4},
$$

where $\varphi$ is a kinematical global time. In particular, in the later case we have for the dynamical pseudometric $\left\{K^{m n}\right\}_{m, n=0,1,2,3}$ :

$$
K_{j m}=\left(J_{j m}+S_{m} \frac{\partial \varphi}{\partial x^{j}}+\frac{\partial \varphi}{\partial x^{m}} S_{j}\right) \quad \forall 0 \leq j, m \leq 3 \quad \forall\left(x^{0}, x^{1}, x^{2}, x^{3}\right) \in \mathbb{R}^{4} .
$$

Moreover, in the latter case we have

$$
\sum_{m=0}^{3} \sum_{n=0}^{3} K^{m n} \frac{\partial \varphi}{\partial x^{m}} \frac{\partial \varphi}{\partial x^{n}}=\sum_{m=0}^{3} \sum_{n=0}^{3} J^{m n} \frac{\partial \varphi}{\partial x^{m}} \frac{\partial \varphi}{\partial x^{n}}=1 \quad \forall\left(x^{0}, x^{1}, x^{2}, x^{3}\right) \in \mathbb{R}^{4},
$$

both triples $\left\{J^{m n}\right\}_{m, n=0,1,2,3},\left\{\Lambda^{m n}\right\}_{m, n=0,1,2,3}, \varphi$, and $\left\{K^{m n}\right\}_{m, n=0,1,2,3},\left\{\Lambda^{m n}\right\}_{m, n=0,1,2,3}, \varphi$ are super-correlated, the dynamical global time equals to kinematical global time $\varphi$ and the dynamical tensor of three-dimensional Geometry $\left\{\Lambda^{m n}\right\}_{m, n=0,1,2,3}$ equals to the kinematical tensor of threedimensional Geometry $\left\{\Theta^{m n}\right\}_{m, n=0,1,2,3}$, so that

$$
\Lambda^{m n}=\Theta^{m n}:=\left(\sum_{j=0}^{3} J^{m j} \frac{\partial \varphi}{\partial x^{j}}\right)\left(\sum_{j=0}^{3} J^{n j} \frac{\partial \varphi}{\partial x^{j}}\right)-J^{m n} \quad \forall m, n=0,1,2,3 .
$$




\subsection{Lagrangian of the genuine gravity}

The model of genuine gravity, we present here, is described by kinematically correlated pseudometric with time-direction $\left(w_{0}, w_{1}, w_{2}, w_{3}\right)$, corresponding to the covector of genuine gravity $\left(S_{0}, S_{1}, S_{2}, S_{3}\right)$ (see Definition 2.21). In order to describe it, consider the strongly-correlated kinematical contravariant pseudo-metric $\left\{J^{m n}\right\}_{m, n=0,1,2,3}$ and the kinematical global time $\varphi$, forming a standard kinematical Lorentz's structure with global time on $\mathbb{R}^{4}$, as in Proposition 2.7 and Remark 2.6. Furthermore, let $\left\{J_{m n}\right\}_{m, n=0,1,2,3}$ be the inverse covariant pseudo-metric on $\mathbb{R}^{4}$, associated with $\left\{J^{m n}\right\}_{m, n=0,1,2,3}$. Given, an arbitrary dynamical four-covector of the time direction, $\left(w_{0}, w_{1}, w_{2}, w_{3}\right)$ (formally unrelated to the kinematical global time $\varphi$ ), which is weakly correlated with $\left\{J^{m n}\right\}_{m, n=0,1,2,3}$, satisfying

$$
\sum_{j=0}^{3} \sum_{m=0}^{3} J^{j m} w_{j} w_{m}>0 \quad \forall\left(x^{0}, x^{1}, x^{2}, x^{3}\right) \in \mathbb{R}^{4},
$$

and a four-covector field $\left(S_{0}, S_{1}, S_{2}, S_{3}\right)$, which we called the four-covector of genuine gravity, consider the two-times covariant tensor $\left\{K_{m n}\right\}_{m, n=0,1,2,3}$ defined by:

$$
K_{j m}=\left(J_{j m}+w_{j} S_{m}+w_{m} S_{j}\right) \quad \forall 0 \leq j, m \leq 3 \quad \forall\left(x^{0}, x^{1}, x^{2}, x^{3}\right) \in \mathbb{R}^{4},
$$

and assume that $\left(S_{0}, S_{1}, S_{2}, S_{3}\right)$ is such that $\left\{K_{m n}\right\}_{m, n=0,1,2,3}$ in (2.198) satisfies

$$
\operatorname{det}\left(\left\{K_{m n}\right\}_{m, n=0,1,2,3}\right)=\operatorname{det}\left(\left\{J_{m n}\right\}_{m, n=0,1,2,3}\right) \quad \forall\left(x^{0}, x^{1}, x^{2}, x^{3}\right) \in \mathbb{R}^{4},
$$

so that $\left(S_{0}, S_{1}, S_{2}, S_{3}\right)$ has only three independent components. Then, as before, the inverse to $\left\{K_{m n}\right\}_{m, n=0,1,2,3}$ two-times contravariant tensor $\left\{K^{m n}\right\}_{m, n=0,1,2,3}$ is a kinematically correlated pseudometric with time-direction $\left(w_{0}, w_{1}, w_{2}, w_{3}\right)$, corresponding to the covector of genuine gravity $\left(S_{0}, S_{1}, S_{2}, S_{3}\right)$. Next, consider a Lagrangian density of the genuine gravitational field as:

$$
\begin{aligned}
& \mathcal{L}_{g}\left(\left(S_{0}, S_{1}, S_{2}, S_{3}\right),\left\{K^{m n}\right\}_{m, n=0,1,2,3}\right):= \\
& \frac{1}{4 \pi G}\left(\sum_{n=0}^{3} \sum_{k=0}^{3} \sum_{m=0}^{3} \sum_{p=0}^{3} \frac{1}{4} K^{m n} K^{p k}\left(\frac{\partial S_{p}}{\partial x^{m}}-\frac{\partial S_{m}}{\partial x^{p}}\right)\left(\frac{\partial S_{k}}{\partial x^{n}}-\frac{\partial S_{n}}{\partial x^{k}}\right)\right)
\end{aligned}
$$

where $G$ is the gravitational constant. Correspondingly, given a subregion $\mathcal{V} \subset \mathbb{R}^{4}$, the Lagrangian of the genuine gravitational field in this subregion, as usual, is given by

$$
\begin{aligned}
& L_{g}\left(S_{0}, S_{1}, S_{2}, S_{3}\right):= \\
& \iiint \int_{\mathcal{V}}\left(\mathcal{L}_{g}\left(\left(S_{0}, S_{1}, S_{2}, S_{3}\right),\left\{K^{m n}\right\}_{m, n=0,1,2,3}\right)\left|\operatorname{det}\left(\left\{K^{m n}\right\}_{n, m=0,1,2,3}\right)\right|^{-\frac{1}{2}}\right) d x^{0} d x^{1} d x^{2} d x^{3} .
\end{aligned}
$$


Moreover, we combine this gravitational Lagrangian with the Lagrangian for the time direction, given by

$$
\begin{aligned}
& L_{R}\left(\left(w_{0}, w_{1}, w_{2}, w_{3}\right)\right):= \\
& \qquad \iiint_{\mathcal{V}}\left(\mathcal{L}_{R}\left(\left(w_{0}, w_{1}, w_{2}, w_{3}\right)\right)\left|\operatorname{det}\left(\left\{J^{m n}\right\}_{n, m=0,1,2,3}\right)\right|^{-\frac{1}{2}}\right) d x^{0} d x^{1} d x^{2} d x^{3},
\end{aligned}
$$

where, the Lagrangian density $\mathcal{L}_{R}$ is given, similarly to (2.177), by

$$
\begin{array}{r}
\mathcal{L}_{R}\left(\left(w_{0}, w_{1}, w_{2}, w_{3}\right)\right):=-\lambda \alpha \mu\left(\sum_{n=0}^{3} \sum_{k=0}^{3} \sum_{m=0}^{3} \sum_{p=0}^{3} \frac{1}{4} J^{m n} J^{p k}\left(\frac{\partial w_{p}}{\partial x^{m}}-\frac{\partial w_{m}}{\partial x^{p}}\right)\left(\frac{\partial w_{k}}{\partial x^{n}}-\frac{\partial w_{n}}{\partial x^{k}}\right)\right) \\
-\frac{\alpha \nu}{4}\left(\sum_{m=0}^{3} \sum_{n=0}^{3} J^{m n} w_{m} w_{n}-1\right)^{2},
\end{array}
$$

where $\mu \neq 0, \nu \neq 0$ are two real constants, and $\alpha \neq 0, \lambda \neq 0$ are two real dimensionless constants, such that

$$
|\alpha| \gg 1 \quad \text { and } \quad|\lambda| \gg 1 .
$$

Furthermore, we combine these Lagrangians with the Lagrangian of the Electromagnetical field, given by

$$
\begin{aligned}
& L_{e}\left(A_{0}, A_{1}, A_{2}, A_{3}\right):= \\
& \iiint \int_{\mathcal{V}}\left(\mathcal{L}_{e}\left(\left(A_{0}, A_{1}, A_{2}, A_{3}\right),\left\{K^{m n}\right\}_{m, n=0,1,2,3}\right)\left|\operatorname{det}\left(\left\{K^{m n}\right\}_{n, m=0,1,2,3}\right)\right|^{-\frac{1}{2}}\right) d x^{0} d x^{1} d x^{2} d x^{3},
\end{aligned}
$$

where, similarly to $(2.112)$ we consider

$$
\begin{aligned}
\mathcal{L}_{e}\left(\left(A_{0}, A_{1}, A_{2}, A_{3}\right),\left\{K^{m n}\right\}_{m, n=0,1,2,3}\right):= \\
-\frac{1}{4 \pi}\left(\sum_{n=0}^{3} \sum_{k=0}^{3} \sum_{m=0}^{3} \sum_{p=0}^{3} \frac{1}{4} K^{m n} K^{p k}\left(\frac{\partial A_{p}}{\partial x^{m}}-\frac{\partial A_{m}}{\partial x^{p}}\right)\left(\frac{\partial A_{k}}{\partial x^{n}}-\frac{\partial A_{n}}{\partial x^{k}}\right)\right) .
\end{aligned}
$$

Finally, we combine all these Lagrangians with the Lagrangian of the real matter, given by

$$
\begin{aligned}
& L_{M}\left(\left\{K_{m n}\right\}_{m, n=0,1,2,3}\right):=-\iiint \int_{\mathcal{V}}\left(\left(\sum_{n=0}^{3} A_{n} j^{n}\right)\left|\operatorname{det}\left(\left\{K^{m n}\right\}_{n, m=0,1,2,3}\right)\right|^{-\frac{1}{2}}\right) d x^{0} d x^{1} d x^{2} d x^{3} \\
+ & \iiint \int_{\mathcal{V}}\left(\mathcal{L}_{M}\left(\left(\left\{K_{m n}\right\}_{m, n=0,1,2,3}\right),\left(x^{0}, x^{1}, x^{2}, x^{3}\right)\right)\left|\operatorname{det}\left(\left\{K^{m n}\right\}_{n, m=0,1,2,3}\right)\right|^{-\frac{1}{2}}\right) d x^{0} d x^{1} d x^{2} d x^{3},
\end{aligned}
$$

where, in the presence of a matter, consisting of $N$ classical point particles $\forall j=1,2, \ldots, N$, with the inertial mass $m_{j}$ and the charge $\sigma_{j}$ for the $j$-th particle and having the four-dimensional space-time trajectory $\chi_{j}\left(s_{j}\right):=\left(\chi_{j}^{0}\left(s_{j}\right), \chi_{j}^{1}\left(s_{j}\right), \chi_{j}^{2}\left(s_{j}\right), \chi_{j}^{3}\left(s_{j}\right)\right):\left[a_{j}, b_{j}\right] \rightarrow \mathbb{R}^{4}$ of the $j$-th particle, parameterized 
by some proper parametrization $s_{j} \in\left[a_{j}, b_{j}\right] \forall j=1,2, \ldots, N$, and given for all instances of time from $-\infty$ to $+\infty$ so that

$$
\lim _{s_{k} \rightarrow a_{k}^{-}} \sum_{j=0}^{3}\left(\left(\chi_{k}^{j}\left(s_{k}\right)\right)^{2}\right)=\lim _{s_{k} \rightarrow b_{k}^{+}} \sum_{j=0}^{3}\left(\left(\chi_{k}^{j}\left(s_{k}\right)\right)^{2}\right)=+\infty \quad \forall k=1,2, \ldots, N,
$$

we consider the total density of the Lagrangian of the given matter $\mathcal{L}_{M}$ by

$$
\begin{array}{r}
\mathcal{L}_{M}\left(\left(\left\{K_{m n}\right\}_{m, n=0,1,2,3}\right),\left(x^{0}, x^{1}, x^{2}, x^{3}\right)\right):=\sum_{k=1}^{N} \int_{a_{k}}^{b_{k}}\{ \\
\left.\left|\operatorname{det}\left\{K^{p q}\left(\chi_{k}\left(s_{k}\right)\right)\right\}\right|^{\frac{1}{2}} W_{L_{\mathcal{G}}, k}\left(s_{k}\right)\left(\sum_{j=0}^{3} w_{j}\left(\chi_{k}\left(s_{k}\right)\right) \frac{d \chi_{k}^{j}}{d s_{k}}\left(s_{k}\right)\right) \delta\left(x^{0}-\chi_{k}^{0}\left(s_{k}\right), \ldots, x^{3}-\chi_{k}^{3}\left(s_{k}\right)\right)\right\} d s_{k} \\
\forall\left(x^{0}, x^{1}, x^{2}, x^{3}\right) \in \mathbb{R}^{4}, \quad \text { (2.209) }
\end{array}
$$

where $W_{L_{\mathcal{G}}, k}\left(s_{k}\right)$ is given by

$$
W_{L_{\mathcal{G}}, k}\left(s_{k}\right):=-m_{k} \mathcal{G}\left(\sum_{j=0}^{3} \sum_{m=0}^{3} K_{j m}\left(\chi_{k}\left(s_{k}\right)\right) u_{k}^{j}\left(s_{k}\right) u_{k}^{m}\left(s_{k}\right)\right) \quad \forall s_{k} \in\left[a_{k}, b_{k}\right] \quad \forall k=1,2, \ldots, N,
$$

where $\left(u_{k}^{0}, u_{k}^{1}, u_{k}^{2}, u_{k}^{3}\right)\left(s_{k}\right):\left[a_{k}, b_{k}\right] \rightarrow \mathbb{R}^{4}$ is the contravariant four-vector of the four-dimensional velocity of the $k$-th particle, given by

$$
\begin{aligned}
& u_{k}^{j}\left(s_{k}\right)=\left(\sum_{m=0}^{3} w_{m}\left(\chi_{k}\left(s_{k}\right)\right) \frac{d \chi_{k}^{m}}{d s_{k}}\left(s_{k}\right)\right)^{-1} \frac{d \chi_{k}^{j}}{d s_{k}}\left(s_{k}\right) \\
& \forall j=0,1,2,3 \quad \forall s_{k} \in\left[a_{k}, b_{k}\right] \quad \forall k=1,2, \ldots, N,
\end{aligned}
$$

$\delta(\cdot)$ is the delta of Dirac in $\mathbb{R}^{4}$, and

$$
\begin{array}{r}
j^{m}\left(x^{0}, x^{1}, x^{2}, x^{3}\right)= \\
\sum_{k=1}^{N} \int_{a_{k}}^{b_{k}}\left\{\left|\operatorname{det}\left\{K^{p q}\left(\chi_{k}\left(s_{k}\right)\right)\right\}\right|^{\frac{1}{2}} \sigma_{k} \frac{d \chi_{k}^{m}}{d s_{k}}\left(s_{k}\right)\left(s_{k}\right) \delta\left(x^{0}-\chi_{k}^{0}\left(s_{k}\right), \ldots, x^{3}-\chi_{k}^{3}\left(s_{k}\right)\right)\right\} d s_{k} \\
\forall\left(x^{0}, x^{1}, x^{2}, x^{3}\right) \in \mathbb{R}^{4} \quad \forall k=1,2, \ldots, N .
\end{array}
$$

As before, here we consider two cases:

- The case of relativistic particles, where $\mathcal{G}(\tau):=\sqrt{\tau}-1$.

- The case of non-relativistic approximation, where $\mathcal{G}(\tau):=\frac{1}{2}(\tau-1)$. 
Therefore, taking into account (2.199), the total Lagrangian of the interaction of the Gravitational and Electromagnetical fields with the matter is given by the following:

$$
\begin{aligned}
& L_{\text {total }}\left(\left(w_{0}, w_{1}, w_{2}, w_{3}\right),\left(S_{0}, S_{1}, S_{2}, S_{3}\right),\left(A_{0}, A_{1}, A_{2}, A_{3}\right)\right):=\iiint \int\{ \\
& \} d x^{0} d x^{1} d x^{2} d x^{3}=\iiint \int\left\{\begin{array}{r}
\mathcal{V} \\
\mathcal{L}_{\text {total }}\left(\left(w_{0}, \ldots, w_{3}\right),\left(S_{0}, \ldots, S_{3}\right),\left(A_{0}, \ldots, A_{3}\right),\left(x^{0}, \ldots, x^{3}\right)\right)\left|\operatorname{det}\left(\left\{J^{m n}\right\}_{n, m=0,1,2,3}\right)\right|^{-\frac{1}{2}} \\
\mathcal{L}_{\text {total }}\left(\left(w_{0}, \ldots, w_{3}\right),\left(S_{0}, \ldots, S_{3}\right),\left(A_{0}, \ldots, A_{3}\right),\left(x^{0}, \ldots, x^{3}\right)\right)\left|\operatorname{det}\left(\left\{K^{m n}\right\}_{n, m=0,1,2,3}\right)\right|^{-\frac{1}{2}} \\
\} d x^{0} d x^{1} d x^{2} d x^{3}, \quad
\end{array}\right.
\end{aligned}
$$

where the total Lagrangian density $\mathcal{L}_{\text {total }}\left(\left(w_{0}, \ldots, w_{3}\right),\left(S_{0}, \ldots, S_{3}\right),\left(A_{0}, \ldots, A_{3}\right),\left(x^{0}, \ldots, x^{3}\right)\right)$ is given by:

$$
\begin{aligned}
& \mathcal{L}_{\text {total }}\left(\left(w_{0}, \ldots, w_{3}\right),\left(S_{0}, \ldots, S_{3}\right),\left(A_{0}, \ldots, A_{3}\right),\left(x^{0}, \ldots, x^{3}\right)\right):= \\
& \mathcal{L}_{R}\left(\left(w_{0}, w_{1}, w_{2}, w_{3}\right)\right)+\mathcal{L}_{g}\left(\left(S_{0}, S_{1}, S_{2}, S_{3}\right),\left\{K^{m n}\right\}_{m, n=0,1,2,3}\right)-\left(\sum_{k=0}^{3} j^{k} A_{k}\right) \\
& +\mathcal{L}_{e}\left(\left(A_{0}, A_{1}, A_{2}, A_{3}\right),\left\{K^{m n}\right\}_{m, n=0,1,2,3}\right)+\mathcal{L}_{M}\left(\left(\left\{K_{m n}\right\}_{m, n=0,1,2,3}\right),\left(x^{0}, x^{1}, x^{2}, x^{3}\right)\right)
\end{aligned}
$$

so that, we have:

$$
\begin{gathered}
\mathcal{L}_{\text {total }}\left(\left(w_{0}, \ldots, w_{3}\right),\left(S_{0}, \ldots, S_{3}\right),\left(A_{0}, \ldots, A_{3}\right),\left(x^{0}, \ldots, x^{3}\right)\right):= \\
-\lambda \alpha \mu\left(\sum_{n=0}^{3} \sum_{k=0}^{3} \sum_{m=0}^{3} \sum_{p=0}^{3} \frac{1}{4} J^{m n} J^{p k}\left(\frac{\partial w_{p}}{\partial x^{m}}-\frac{\partial w_{m}}{\partial x^{p}}\right)\left(\frac{\partial w_{k}}{\partial x^{n}}-\frac{\partial w_{n}}{\partial x^{k}}\right)\right) \\
-\frac{\alpha \nu}{2}\left(\sum_{m=0}^{3} \sum_{n=0}^{3} J^{m n} w_{m} w_{n}-1\right)^{2}+\mathcal{L}_{M}\left(\left(\left\{K_{m n}\right\}_{m, n=0,1,2,3}\right),\left(x^{0}, x^{1}, x^{2}, x^{3}\right)\right) \\
+\frac{1}{4 \pi G}\left(\sum_{n=0}^{3} \sum_{k=0}^{3} \sum_{m=0}^{3} \sum_{p=0}^{3} \frac{1}{4} K^{m n} K^{p k}\left(\frac{\partial S_{p}}{\partial x^{m}}-\frac{\partial S_{m}}{\partial x^{p}}\right)\left(\frac{\partial S_{k}}{\partial x^{n}}-\frac{\partial S_{n}}{\partial x^{k}}\right)\right) \\
-\frac{1}{4 \pi}\left(\sum_{n=0}^{3} \sum_{k=0}^{3} \sum_{m=0}^{3} \sum_{p=0}^{3} \frac{1}{4} K^{m n} K^{p k}\left(\frac{\partial A_{p}}{\partial x^{m}}-\frac{\partial A_{m}}{\partial x^{p}}\right)\left(\frac{\partial A_{k}}{\partial x^{n}}-\frac{\partial A_{n}}{\partial x^{k}}\right)\right)-\left(\sum_{k=0}^{3} j^{k} A_{k}\right) .
\end{gathered}
$$

Furthermore, in the case where:

$$
|\alpha| \rightarrow+\infty \quad \text { and } \quad|\lambda| \rightarrow+\infty,
$$

we greatly simplify the total Lagrangian, since, then, by (2.193) we have

$$
\left(w_{0}, w_{1}, w_{2}, w_{3}\right)=\left(\frac{\partial \varphi}{\partial x^{0}}, \frac{\partial \varphi}{\partial x^{1}}, \frac{\partial \varphi}{\partial x^{2}}, \frac{\partial \varphi}{\partial x^{3}}\right) \quad \forall\left(x^{0}, x^{1}, x^{2}, x^{3}\right) \in \mathbb{R}^{4},
$$

where $\varphi$ is a kinematical global time, satisfying

$$
\sum_{m=0}^{3} \sum_{n=0}^{3} J^{m n} \frac{\partial \varphi}{\partial x^{m}} \frac{\partial \varphi}{\partial x^{n}}=1 \quad \forall\left(x^{0}, x^{1}, x^{2}, x^{3}\right) \in \mathbb{R}^{4} .
$$


Thus, $\left(w_{0}, w_{1}, w_{2}, w_{3}\right)$ is fixed and it does not varies in the Lagrangian, and we can write:

$$
\begin{aligned}
& L_{\text {total }}\left(\left(S_{0}, S_{1}, S_{2}, S_{3}\right),\left(A_{0}, A_{1}, A_{2}, A_{3}\right)\right):= \\
& \iiint \int_{\mathcal{V}} \mathcal{L}_{\text {total }}\left(\left(S_{0}, \ldots, S_{3}\right),\left(A_{0}, \ldots, A_{3}\right),\left(x^{0}, \ldots, x^{3}\right)\right)\left|\operatorname{det}\left(\left\{J^{m n}\right\}_{n, m=0,1,2,3}\right)\right|^{-\frac{1}{2}} d x^{0} d x^{1} d x^{2} d x^{3}= \\
& \iiint \int_{\mathcal{V}} \mathcal{L}_{\text {total }}\left(\left(S_{0}, \ldots, S_{3}\right),\left(A_{0}, \ldots, A_{3}\right),\left(x^{0}, \ldots, x^{3}\right)\right)\left|\operatorname{det}\left(\left\{K^{m n}\right\}_{n, m=0,1,2,3}\right)\right|^{-\frac{1}{2}} d x^{0} d x^{1} d x^{2} d x^{3},
\end{aligned}
$$

where the total Lagrangian density $\mathcal{L}_{\text {total }}\left(\left(S_{0}, \ldots, S_{3}\right),\left(A_{0}, \ldots, A_{3}\right),\left(x^{0}, \ldots, x^{3}\right)\right)$ is given by:

$$
\begin{gathered}
\mathcal{L}_{\text {total }}\left(\left(S_{0}, \ldots, S_{3}\right),\left(A_{0}, \ldots, A_{3}\right),\left(x^{0}, \ldots, x^{3}\right)\right):=\mathcal{L}_{M}\left(\left(\left\{K_{m n}\right\}_{m, n=0,1,2,3}\right),\left(x^{0}, x^{1}, x^{2}, x^{3}\right)\right) \\
+\frac{1}{4 \pi G}\left(\sum_{n=0}^{3} \sum_{k=0}^{3} \sum_{m=0}^{3} \sum_{p=0}^{3} \frac{1}{4} K^{m n} K^{p k}\left(\frac{\partial S_{p}}{\partial x^{m}}-\frac{\partial S_{m}}{\partial x^{p}}\right)\left(\frac{\partial S_{k}}{\partial x^{n}}-\frac{\partial S_{n}}{\partial x^{k}}\right)\right) \\
-\frac{1}{4 \pi}\left(\sum_{n=0}^{3} \sum_{k=0}^{3} \sum_{m=0}^{3} \sum_{p=0}^{3} \frac{1}{4} K^{m n} K^{p k}\left(\frac{\partial A_{p}}{\partial x^{m}}-\frac{\partial A_{m}}{\partial x^{p}}\right)\left(\frac{\partial A_{k}}{\partial x^{n}}-\frac{\partial A_{n}}{\partial x^{k}}\right)\right)-\left(\sum_{k=0}^{3} j^{k} A_{k}\right),
\end{gathered}
$$

with

$$
K_{j m}=\left(J_{j m}+S_{m} \frac{\partial \varphi}{\partial x^{j}}+\frac{\partial \varphi}{\partial x^{m}} S_{j}\right) \quad \forall 0 \leq j, m \leq 3 \quad \forall\left(x^{0}, x^{1}, x^{2}, x^{3}\right) \in \mathbb{R}^{4},
$$

and a four-covector field of genuine gravity $\left(S_{0}, S_{1}, S_{2}, S_{3}\right)$ satisfies the restriction:

$$
\operatorname{det}\left(\left\{K_{m n}\right\}_{m, n=0,1,2,3}\right)=\operatorname{det}\left(\left\{J_{m n}\right\}_{m, n=0,1,2,3}\right) \quad \forall\left(x^{0}, x^{1}, x^{2}, x^{3}\right) \in \mathbb{R}^{4},
$$

so that $\left(S_{0}, S_{1}, S_{2}, S_{3}\right)$ has only three independent components.

Note that, since the full Lagrangian in (2.213), (2.215) is independent on the kinematical global time $\varphi$ (it depends only on the dynamical time direction $\left(w_{0}, w_{1}, w_{2}, w_{3}\right)$ ), then a Lorentzian coordinate systems, where we have

$$
\begin{cases}J^{00}=1 & \\ J^{0 j}=J^{j 0}=0 & \forall j=1,2,3 \\ J^{j m}:=-\delta_{j m} & \forall j, m=1,2,3,\end{cases}
$$

are the most convenient coordinate systems, to operate with the full Lagrangian. On the other hand, in the limiting case (2.216), where we consider the simplified Lagrangian in (2.219) (2.220), cartesian coordinate systems are often more convenient, than Lorentzian, since in the limiting case we have $\left(w_{0}, w_{1}, w_{2}, w_{3}\right)=\left(\frac{\partial \varphi}{\partial x^{0}}, \frac{\partial \varphi}{\partial x^{1}}, \frac{\partial \varphi}{\partial x^{2}}, \frac{\partial \varphi}{\partial x^{3}}\right)$, and at the same time in the cartesian coordinate systems we always have $\varphi=x^{0}+$ Const, so that we obtain $\left(w_{0}, w_{1}, w_{2}, w_{3}\right)=(1,0,0,0)$. Note also that by Lemma 11.5 from the Appendix in an arbitrary cartesian coordinate system we still have

$$
\operatorname{det}\left(\left\{K_{m n}\right\}_{m, n=0,1,2,3}\right)=\operatorname{det}\left(\left\{J_{m n}\right\}_{m, n=0,1,2,3}\right)=-1 \quad \forall\left(x^{0}, x^{1}, x^{2}, x^{3}\right) \in \mathbb{R}^{4} .
$$


In the following sections we prove, in particular, that the Gravitational field, governed by the simplified Lagrangian in (2.219) (2.220), generated by some massive body, resting and spherically symmetric in some $\underline{\text { cartesian }}$ and inertial coordinate system, is given by the pseudo-metric $\left\{K_{m n}\right\}_{m, n=0,1,2,3}$, such that there exists some curvilinear (non-cartesian) coordinate system in $\mathbb{R}^{4}$, where $\left\{K_{m n}\right\}_{m, n=0,1,2,3}$ coincides with the well known Schwarzschild metric from the General Relativity! In particular, all the optical effects that we find in the framework of our model coincide with the effects considered in the framework of General Relativity for the Schwarzschild metric. In particular, the Michelson-Morely experiment and all Sagnac-type effects will lead to the same result in the framework of our model like in the case of the General relativity. Moreover, since the Maxwell equations in both models have the same tensor form, all the electromagnetic effects, where the time does not appear explicitly will be the same. Similarly, the curvature of the light path in the Sun's gravitational field will be the same in both models. Finally, in the particular case of $\mathcal{G}(\tau)=\sqrt{\tau}$, i.e. in the case of the relativistic Lagrangian of the motion in (2.102) all the mechanical effects will be the same in the framework of our model like in the case of the General relativity for the Schwarzschild metric, provided that the time does not appear explicitly in this effects. In particular, the movement of the Mercury planet in the Sun's gravitational field will be the same in both models.

Furthermore, we also prove that Gravitational field, governed by the simplified Lagrangian in (2.219) (2.220), generated by a general slowly (non-relativistically) moving massive matter in some cartesian coordinate system, can be well approximated, by the classical model of the Newtonian Gravity.

Note here about the following advantage of the presented here model of gravity with respect to the usual Theory of General Relativity. The simplified Lagrangian in (2.219) (2.220) for the gravity depends only on four-component field $\left(S_{0}, S_{1}, S_{2}, S_{3}\right)$, which is by (2.199) has only three independent components. Even the full Lagrangian in (2.213), (2.215), dependent only on $\left(S_{0}, S_{1}, S_{2}, S_{3}\right)$ and $\left(w_{0}, w_{1}, w_{2}, w_{3}\right)$, that is by (2.199) has only seven independent components. On the other hand, in the General Relativity the symmetric tensor $\left\{K_{m n}\right\}_{m, n=0,1,2,3}$ has all ten independent components that makes the corresponding system of partial differential equations to be much more complicated.

Finally, in section 9 we give the covariant formulation of the Electrodynamics of the moving dielectric and para/dia-magnetic continuum mediums in arbitrary dynamical pseudo-metric. The Lorentz's covariant theory of the moving para/dia-magnetic continuum mediums in the flat Lorentz's pseudo-metric was first introduced in [8] by H. Minkowski (1908). Here we formulate the generally covariant theory in the different alternative way, that suite to formulate it in a general pseudo-metric including the presence of the genuine gravity. 


\section{Mass, charge and Lagrangian densities and currents of the system of classical point particles}

Definition 3.1. Consider a classical point particle with the inertial mass $m$ and the charge $\sigma$ moving in the generalized-gravitational field, given by a contravariant pseudo-metric $\left\{K^{m n}\right\}_{m, n=0,1,2,3}$, which is correlated with a four-covector of the time direction $\left(w_{0}, w_{1}, w_{2}, w_{3}\right)$, and influenced by the electromagnetic field with the four-covector of the electromagnetic potential $\left(A_{0}, A_{1}, A_{2}, A_{3}\right)$. Next, assume that $\chi(s):=\left(\chi^{0}(s), \chi^{1}(s), \chi^{2}(s), \chi^{3}(s)\right):[a, b] \rightarrow \mathbb{R}^{4}$ is a four-dimensional space-time trajectory of the particle, parameterized by some proper parametrization $s \in[a, b]$ (including the cases where $a=-\infty$ and/or $b=+\infty)$. Moreover, assume that the infinite trajectory of the motion is considered for all instances of time from $-\infty$ to $+\infty$ so that

$$
\lim _{s \rightarrow a^{-}} \sum_{j=0}^{3}\left(\left(\chi^{j}(s)\right)^{2}\right)=\lim _{s \rightarrow b^{+}} \sum_{j=0}^{3}\left(\left(\chi^{j}(s)\right)^{2}\right)=+\infty .
$$

Next, given an arbitrary covariant (contravariant) scalar quantity $W(s):[a, b] \rightarrow \mathbb{R}$, defined across the trajectory of motion with the chosen proper parametrization, consider a four-current density $\left(I_{W}^{0}, I_{W}^{1}, I_{W}^{2}, I_{W}^{3}\right)$ of the quantity $W$ and a scalar density $\rho_{W}$ of the quantity $W$ as generalized functions (distributions), defined by

$$
\begin{aligned}
& I_{W}^{j}\left(x^{0}, x^{1}, x^{2}, x^{3}\right):= \\
& \int_{a}^{b}\left|\operatorname{det}\left(\left\{K^{m n}(\chi(s))\right\}_{n, m=0,1,2,3}\right)\right|^{\frac{1}{2}} W(s) \frac{d \chi^{j}}{d s}(s) \delta\left(x^{0}-\chi^{0}(s), \ldots, x^{3}-\chi^{3}(s)\right) d s \\
& =\left|\operatorname{det}\left(\left\{K^{m n}\left(x^{0}, x^{1}, x^{2}, x^{3}\right)\right\}_{n, m=0,1,2,3}\right)\right|^{\frac{1}{2}} \int_{a}^{b} W(s) \frac{d \chi^{j}}{d s}(s) \delta\left(x^{0}-\chi^{0}(s), \ldots, x^{3}-\chi^{3}(s)\right) d s \\
& \forall j=0,1,2,3 \quad \forall\left(x^{0}, x^{1}, x^{2}, x^{3}\right) \in \mathbb{R}^{4}, \quad(3.2)
\end{aligned}
$$

and

$$
\begin{aligned}
& \rho_{W}\left(x^{0}, x^{1}, x^{2}, x^{3}\right):= \\
& \int_{a}^{b}\left|\operatorname{det}\left(\left\{K^{m n}(\chi(s))\right\}_{n, m=0,1,2,3}\right)\right|^{\frac{1}{2}} W(s)\left(\sum_{j=0}^{3} w_{j}(\chi(s)) \frac{d \chi^{j}}{d s}(s)\right) \delta\left(x^{0}-\chi^{0}(s), \ldots, x^{3}-\chi^{3}(s)\right) d s= \\
& \sum_{j=0}^{3}\left(\left|\operatorname{det}\left(\left\{K^{m n}\right\}_{n, m=0,1,2,3}\right)\right|^{\frac{1}{2}} w_{j}\right)\left(x^{0}, x^{1}, x^{2}, x^{3}\right) \int_{a}^{b} W(s) \frac{d \chi^{j}}{d s}(s) \delta\left(x^{0}-\chi^{0}(s), \ldots, x^{3}-\chi^{3}(s)\right) d s \\
& \forall\left(x^{0}, x^{1}, x^{2}, x^{3}\right) \in \mathbb{R}^{4}, \quad(3.3)
\end{aligned}
$$

so that

$$
\rho_{W}\left(x^{0}, x^{1}, x^{2}, x^{3}\right):=\sum_{j=0}^{3} w_{j}\left(x^{0}, x^{1}, x^{2}, x^{3}\right) I_{W}^{j}\left(x^{0}, x^{1}, x^{2}, x^{3}\right) \quad \forall\left(x^{0}, x^{1}, x^{2}, x^{3}\right) \in \mathbb{R}^{4} .
$$


Here, given a point $\left(a^{0}, a^{1}, a^{2}, a^{3}\right) \in \mathbb{R}^{4}, \delta\left(x^{0}-a^{0}, \ldots, x^{3}-a^{3}\right)$ is a four-dimensional scalar deltafunction of the point $\left(a^{0}, a^{1}, a^{2}, a^{3}\right)$ (the discrete unit measure of the point $\left.\left(a^{0}, a^{1}, a^{2}, a^{3}\right) \in \mathbb{R}^{4}\right)$. Note that obviously, $\left(I_{W}^{0}, I_{W}^{1}, I_{W}^{2}, I_{W}^{3}\right)$ in (3.2) is a valid contravariant four-vector field and $\rho_{W}$ in (3.3) is a valid covariant (contravariant) scalar. Note also that, (3.2) and (3.3) are independent on the choice of the proper parametrization. Finally, note that by theory of distributions the definition (3.2) and (3.3) mean that for every smooth scalar classical function with compact support $\xi\left(x^{0}, x^{1}, x^{2}, x^{3}\right) \in C_{c}^{\infty}\left(\mathbb{R}^{4}\right)$ we have

$$
\begin{aligned}
\iiint \int_{\mathbb{R}^{4}} I_{W}^{j}\left(x^{0}, x^{1}, x^{2}, x^{3}\right) \xi\left(x^{0}, x^{1}, x^{2}, x^{3}\right) d x^{0} d x^{1} d x^{2} d x^{3}= \\
\quad \int_{a}^{b}\left|\operatorname{det}\left(\left\{K^{m n}(\chi(s))\right\}_{n, m=0,1,2,3}\right)\right|^{\frac{1}{2}} W(s) \frac{d \chi^{j}}{d s}(s) \xi(\chi(s)) d s \quad \forall j=0,1,2,3,
\end{aligned}
$$

and

$$
\begin{aligned}
\iiint \int_{\mathbb{R}^{4}} \rho_{W}\left(x^{0}, x^{1}, x^{2}, x^{3}\right) \xi\left(x^{0}, x^{1}, x^{2}, x^{3}\right) d x^{0} d x^{1} d x^{2} d x^{3}= \\
\int_{a}^{b}\left|\operatorname{det}\left(\left\{K^{m n}(\chi(s))\right\}_{n, m=0,1,2,3}\right)\right|^{\frac{1}{2}} W(s)\left(\sum_{j=0}^{3} w_{j}(\chi(s)) \frac{d \chi^{j}}{d s}(s)\right) \xi(\chi(s)) d s .
\end{aligned}
$$

Definition 3.2. Given an arbitrary contravariant four-vector field $\left(f^{0}, f^{1}, f^{2}, f^{3}\right)\left(x^{0}, x^{1}, x^{2}, x^{3}\right)$, define the covariant divergence of it with respect to the contravariant pseudo-metric $K:=\left\{K^{m n}\right\}_{m, n=0,1,2,3}$, by the following

$$
\begin{gathered}
\left\{\operatorname{div}\left(f^{0}, f^{1}, f^{2}, f^{3}\right)\right\}_{K}\left(x^{0}, x^{1}, x^{2}, x^{3}\right):= \\
\sum_{j=0}^{3} \frac{\partial f^{j}}{\partial x^{j}}\left(x^{0}, x^{1}, x^{2}, x^{3}\right)+\sum_{j=0}^{3} f^{j}\left(x^{0}, x^{1}, x^{2}, x^{3}\right)\left(\frac{\frac{\partial}{\partial x^{j}}\left(\left|\operatorname{det}\left(\left\{K^{m n}\right\}_{n, m=0,1,2,3}\right)\right|^{-\frac{1}{2}}\right)}{\left|\operatorname{det}\left(\left\{K^{m n}\right\}_{n, m=0,1,2,3}\right)\right|^{-\frac{1}{2}}}\right)\left(x^{0}, x^{1}, x^{2}, x^{3}\right) \\
=\frac{1}{\left|\operatorname{det}\left(\left\{K^{m n}\right\}_{n, m=0,1,2,3}\right)\right|^{-\frac{1}{2}}}\left(\sum_{j=0}^{3} \frac{\partial}{\partial x^{j}}\left\{\left|\operatorname{det}\left(\left\{K^{m n}\right\}_{n, m=0,1,2,3}\right)\right|^{-\frac{1}{2}} f^{j}\right\}\left(x^{0}, x^{1}, x^{2}, x^{3}\right)\right) \\
\forall\left(x^{0}, x^{1}, x^{2}, x^{3}\right) \in \mathbb{R}^{4} .
\end{gathered}
$$

It is well known from Tensor Analysys that if $\left(f^{0}, f^{1}, f^{2}, f^{3}\right)$ is a contravariant four-vector, then $\left\{\operatorname{div}\left(f^{0}, f^{1}, f^{2}, f^{3}\right)\right\}_{K}$ is a valid covariant (contravariant) scalar.

Proposition 3.1. Consider a classical point particle with the inertial mass $m$ and the charge $\sigma$, moving in the generalized-gravitational field, given by a contravariant pseudo-metric $\left\{K^{m n}\right\}_{m, n=0,1,2,3}$, which is correlated with a four-covector of the time direction $\left(w_{0}, w_{1}, w_{2}, w_{3}\right)$, and influenced by the electromagnetic field with the four-covector of the electromagnetic potential $\left(A_{0}, A_{1}, A_{2}, A_{3}\right)$. Next, assume that $\chi(s):=\left(\chi^{0}(s), \chi^{1}(s), \chi^{2}(s), \chi^{3}(s)\right):[a, b] \rightarrow \mathbb{R}^{4} \in \mathbb{R}^{4}$ is a 
four-dimensional space-time trajectory of the particle, parameterized by some proper parametrization $s \in[a, b]$ (including the cases where $a=-\infty$ and/or $b=+\infty$ ). Moreover, assume that the infinite trajectory of the motion is considered for all instances of time from $-\infty$ to $+\infty$ so that

$$
\lim _{s \rightarrow a^{-}} \sum_{j=0}^{3}\left(\left(\chi^{j}(s)\right)^{2}\right)=\lim _{s \rightarrow b^{+}} \sum_{j=0}^{3}\left(\left(\chi^{j}(s)\right)^{2}\right)=+\infty .
$$

Next, consider an arbitrary quantity $W(s):[a, b] \rightarrow \mathbb{R}$, defined across the trajectory of motion with the chosen proper parametrization, and consider a four-current density $\left(I_{W}^{0}, I_{W}^{1}, I_{W}^{2}, I_{W}^{3}\right)$ of the quantity $W$, given by (3.2). Then, in the case when $W(s)$ is a constant across the trajectory of the motion, independent on the parameter $s \in[a, b]$, we have the following conservation of the current:

$$
\left\{\operatorname{div}\left(I_{W}^{0}, I_{W}^{1}, I_{W}^{2}, I_{W}^{3}\right)\right\}_{K}\left(x^{0}, x^{1}, x^{2}, x^{3}\right)=0 \quad \forall\left(x^{0}, x^{1}, x^{2}, x^{3}\right) \in \mathbb{R}^{4},
$$

where the covariant divergence $\{\operatorname{div}(\cdot)\}_{K}$ is defined by (3.7).

Definition 3.3. Consider a system of $N$ classical point particles $\forall j=1,2, \ldots, N$, with the inertial mass $m_{j}$ and the charge $\sigma_{j}$ for the $j$-th particle, moving in the generalized-gravitational field given by a contravariant pseudo-metric $\left\{K^{m n}\right\}_{m, n=0,1,2,3}$, which is correlated with a fourcovector of the time direction $\left(w_{0}, w_{1}, w_{2}, w_{3}\right)$, and influenced by the electromagnetic field with the four-covector of the electromagnetic potential $\left(A_{0}, A_{1}, A_{2}, A_{3}\right)$. Next, assume that $\chi_{j}\left(s_{j}\right):=$ $\left(\chi_{j}^{0}\left(s_{j}\right), \chi_{j}^{1}\left(s_{j}\right), \chi_{j}^{2}\left(s_{j}\right), \chi_{j}^{3}\left(s_{j}\right)\right):\left[a_{j}, b_{j}\right] \rightarrow \mathbb{R}^{4}$ is a four-dimensional space-time trajectory of the $j$-th particle, parameterized by some proper parametrization $s_{j} \in\left[a_{j}, b_{j}\right]$ for the $j$-th particle $\forall j=1,2, \ldots, N$ (including the cases where $a_{j}=-\infty$ and/or $b_{j}=+\infty$ ). Moreover, assume that the infinite trajectory of the motion is considered for all instances of time from $-\infty$ to $+\infty$ so that

$$
\lim _{s_{k} \rightarrow a_{k}^{-}} \sum_{j=0}^{3}\left(\left(\chi_{k}^{j}\left(s_{k}\right)\right)^{2}\right)=\lim _{s_{k} \rightarrow b_{k}^{+}} \sum_{j=0}^{3}\left(\left(\chi_{k}^{j}\left(s_{k}\right)\right)^{2}\right)=+\infty \quad \forall k=1,2, \ldots, N .
$$

Next, for every $k=1,2, \ldots, N$ consider covariant (contravariant) scalar quantities $W_{\sigma_{k}}\left(s_{k}\right)$ : $\left[a_{k}, b_{k}\right] \rightarrow \mathbb{R}$ and $W_{m_{k}}(s):\left[a_{k}, b_{k}\right] \rightarrow \mathbb{R}$, defined across the trajectories of motion with the chosen proper parametrization $s_{k}$ by the following:

$$
\left\{\begin{array}{lll}
W_{\sigma_{k}}\left(s_{k}\right)=\sigma_{k} & \forall s_{k} \in\left[a_{k}, b_{k}\right] & \forall k=1,2, \ldots, N \\
W_{m_{k}}(s)=m_{k} & \forall s_{k} \in\left[a_{k}, b_{k}\right] & \forall k=1,2, \ldots, N
\end{array}\right.
$$

where $\sigma_{k}$ is the charge of the given $k$-th point particle and $m_{k}$ is the mass of the given $k$-th point particle. Then, obviously, both quantities $W_{\sigma_{k}}\left(s_{k}\right):\left[a_{k}, b_{k}\right] \rightarrow \mathbb{R}$ and $W_{m_{k}}(s):\left[a_{k}, b_{k}\right] \rightarrow \mathbb{R}$ are constant across every trajectory of motion of the $k$-th particle, with the chosen proper parametrization $s_{k}$. Moreover, for every $k=1,2, \ldots, N$ consider covariant (contravariant) scalar quantity $W_{L_{\mathcal{G}}, k}(s):\left[a_{k}, b_{k}\right] \rightarrow \mathbb{R}$, related to the general Lagrangian $L_{\mathcal{G}, k}$ of the $k$-th particle in (2.100), and 
defined across the trajectory of motion with the chosen proper parametrization by the following:

$$
\begin{array}{r}
W_{L_{\mathcal{G}}, k}\left(s_{k}\right):=-m_{k} \mathcal{G}\left(\sum_{j=0}^{3} \sum_{m=0}^{3} K_{j m}\left(\chi_{k}\left(s_{k}\right)\right) u_{k}^{j}\left(s_{k}\right) u_{k}^{m}\left(s_{k}\right)\right)-\sum_{j=0}^{3} \sigma_{k} u_{k}^{j}(s) A_{j}\left(\chi_{k}\left(s_{k}\right)\right) \\
\forall s_{k} \in\left[a_{k}, b_{k}\right] \quad \forall k=1,2, \ldots, N,
\end{array}
$$

where $\left(u_{k}^{0}, u_{k}^{1}, u_{k}^{2}, u_{k}^{3}\right)\left(s_{k}\right):\left[a_{k}, b_{k}\right] \rightarrow \mathbb{R}^{4}$ is the contravariant four-vector of the four-dimensional velocity of the $k$-th particle, given as in (2.96), by

$$
\begin{aligned}
& u_{k}^{j}\left(s_{k}\right)=\left(\sum_{m=0}^{3} w_{m}\left(\chi_{k}\left(s_{k}\right)\right) \frac{d \chi_{k}^{m}}{d s_{k}}\left(s_{k}\right)\right)^{-1} \frac{d \chi_{k}^{j}}{d s_{k}}\left(s_{k}\right) \\
& \forall j=0,1,2,3 \quad \forall s_{k} \in\left[a_{k}, b_{k}\right] \quad \forall k=1,2, \ldots, N .
\end{aligned}
$$

Finally, for every $k=1,2, \ldots, N$ consider covariant (contravariant) scalar quantity $\hat{W}_{L_{\mathcal{G}}, k}(s)$ : $\left[a_{k}, b_{k}\right] \rightarrow \mathbb{R}$, related to the general Lagrangian $L_{\mathcal{G}, k}$ of the $k$-th particle in (2.100), and defined across the trajectory of motion with the chosen proper parametrization by the following:

$$
\begin{aligned}
& \hat{W}_{L_{\mathcal{G}}, k}\left(s_{k}\right):=-m_{k} \mathcal{G}\left(\sum_{j=0}^{3} \sum_{m=0}^{3} K_{j m}\left(\chi_{k}\left(s_{k}\right)\right) u_{k}^{j}\left(s_{k}\right) u_{k}^{m}\left(s_{k}\right)\right) \\
& \forall s_{k} \in\left[a_{k}, b_{k}\right] \quad \forall k=1,2, \ldots, N .
\end{aligned}
$$

Then, define the contravariant four-vector of the total charge four-current density $\left(j^{0}, j^{1}, j^{2}, j^{3}\right):=\left(j^{0}, j^{1}, j^{2}, j^{3}\right)\left(x^{0}, x^{1}, x^{2}, x^{3}\right)$ and the total scalar charge density $\rho:=\rho\left(x^{0}, x^{1}, x^{2}, x^{3}\right)$ of the system of the charges by:

$$
\begin{aligned}
& j^{n}\left(x^{0}, x^{1}, x^{2}, x^{3}\right):=\sum_{k=1}^{N} I_{W_{\sigma_{k}}}^{n}\left(x^{0}, x^{1}, x^{2}, x^{3}\right) \quad \forall n=0,1,2,3 \quad \forall\left(x^{0}, x^{1}, x^{2}, x^{3}\right) \in \mathbb{R}^{4}, \\
& \rho\left(x^{0}, x^{1}, x^{2}, x^{3}\right):=\sum_{k=1}^{N} \rho_{W_{\sigma_{k}}}\left(x^{0}, x^{1}, x^{2}, x^{3}\right) \quad \forall\left(x^{0}, x^{1}, x^{2}, x^{3}\right) \in \mathbb{R}^{4},
\end{aligned}
$$

where the contravariant four-vector of the four-current density $\left(I_{W}^{0}, I_{W}^{1}, I_{W}^{2}, I_{W}^{3}\right)$ is given by $(3.2)$ and a scalar density $\rho_{W}$ is given by (3.3), so that

$$
\begin{aligned}
& j^{n}\left(x^{0}, x^{1}, x^{2}, x^{3}\right):= \\
& \sum_{k=1}^{N} \int_{a_{k}}^{b_{k}}\left|\operatorname{det}\left(\left\{K^{p q}\left(\chi_{k}\left(s_{k}\right)\right)\right\}_{p, q=0,1,2,3}\right)\right|^{\frac{1}{2}} \sigma_{k} \frac{d \chi_{k}^{n}}{d s_{k}}\left(s_{k}\right) \delta\left(x^{0}-\chi_{k}^{0}\left(s_{k}\right), \ldots, x^{3}-\chi_{k}^{3}\left(s_{k}\right)\right) d s_{k} \\
& \forall n=0,1,2,3 \quad \forall\left(x^{0}, x^{1}, x^{2}, x^{3}\right) \in \mathbb{R}^{4}, \quad(3.16
\end{aligned}
$$


and

$$
\begin{aligned}
& \rho\left(x^{0}, x^{1}, x^{2}, x^{3}\right):= \\
& \sum_{k=1}^{N} \sigma_{k} \int_{a_{k}}^{b_{k}}\left|\operatorname{det}\left\{K^{p q}\left(\chi_{k}\left(s_{k}\right)\right\}\right)\right|^{\frac{1}{2}}\left(\sum_{j=0}^{3} w_{j}\left(\chi_{k}\left(s_{k}\right)\right) \frac{d \chi_{k}^{j}}{d s_{k}}\left(s_{k}\right)\right) \delta\left(x^{0}-\chi_{k}^{0}\left(s_{k}\right), \ldots, x^{3}-\chi_{k}^{3}\left(s_{k}\right)\right) d s_{k} \\
& \forall\left(x^{0}, x^{1}, x^{2}, x^{3}\right) \in \mathbb{R}^{4} .
\end{aligned}
$$

Then, by Proposition 3.1 we deduce the following conservation of the total charge current:

$$
\left\{\operatorname{div}\left(j^{0}, j^{1}, j^{2}, j^{3}\right)\right\}_{K}\left(x^{0}, x^{1}, x^{2}, x^{3}\right)=0 \quad \forall\left(x^{0}, x^{1}, x^{2}, x^{3}\right) \in \mathbb{R}^{4},
$$

where the covariant divergence $\{\operatorname{div}(\cdot)\}_{K}$ is defined by (3.7). Similarly define the contravariant four-vector of the total mass four-current density

$\left(j_{M}^{0}, j_{M}^{1}, j_{M}^{2}, j_{M}^{3}\right):=\left(j_{M}^{0}, j_{M}^{1}, j_{M}^{2}, j_{M}^{3}\right)\left(x^{0}, x^{1}, x^{2}, x^{3}\right)$ and the total scalar mass density $M:=M\left(x^{0}, x^{1}, x^{2}, x^{3}\right)$ of the system of the masses by:

$$
\begin{aligned}
j_{M}^{n}\left(x^{0}, x^{1}, x^{2}, x^{3}\right):=\sum_{k=1}^{N} I_{W_{m_{k}}}^{n}\left(x^{0}, x^{1}, x^{2}, x^{3}\right) \quad \forall n=0,1,2,3 & \forall\left(x^{0}, x^{1}, x^{2}, x^{3}\right) \in \mathbb{R}^{4}, \\
M\left(x^{0}, x^{1}, x^{2}, x^{3}\right):=\sum_{k=1}^{N} \rho_{W_{m_{k}}}\left(x^{0}, x^{1}, x^{2}, x^{3}\right) & \forall\left(x^{0}, x^{1}, x^{2}, x^{3}\right) \in \mathbb{R}^{4},
\end{aligned}
$$

where the contravariant four-vector of the four-current density $\left(I_{W}^{0}, I_{W}^{1}, I_{W}^{2}, I_{W}^{3}\right)$ is given by $(3.2)$ and a scalar density $\rho_{W}$ is given by $(3.3)$, so that

$$
\begin{aligned}
& j_{M}^{n}\left(x^{0}, x^{1}, x^{2}, x^{3}\right):= \\
& \sum_{k=1}^{N} \int_{a_{k}}^{b_{k}}\left|\operatorname{det}\left(\left\{K^{p q}\left(\chi_{k}\left(s_{k}\right)\right)\right\}_{p, q=0,1,2,3}\right)\right|^{\frac{1}{2}} m_{k} \frac{d \chi_{k}^{n}}{d s_{k}}\left(s_{k}\right) \delta\left(x^{0}-\chi_{k}^{0}\left(s_{k}\right), \ldots, x^{3}-\chi_{k}^{3}\left(s_{k}\right)\right) d s_{k} \\
& \forall n=0,1,2,3 \quad \forall\left(x^{0}, x^{1}, x^{2}, x^{3}\right) \in \mathbb{R}^{4}, \quad(3
\end{aligned}
$$

and

$$
\begin{aligned}
& M\left(x^{0}, x^{1}, x^{2}, x^{3}\right):= \\
& \sum_{k=1}^{N} m_{k} \int_{a_{k}}^{b_{k}}\left|\operatorname{det}\left\{K^{p q}\left(\chi_{k}\left(s_{k}\right)\right)\right\}\right|^{\frac{1}{2}}\left(\sum_{j=0}^{3} w_{j}\left(\chi_{k}\left(s_{k}\right)\right) \frac{d \chi_{k}^{j}}{d s_{k}}\left(s_{k}\right)\right) \delta\left(x^{0}-\chi_{k}^{0}\left(s_{k}\right), \ldots, x^{3}-\chi_{k}^{3}\left(s_{k}\right)\right) d s_{k} \\
& \forall\left(x^{0}, x^{1}, x^{2}, x^{3}\right) \in \mathbb{R}^{4} .
\end{aligned}
$$

Then, as before, by Proposition 3.1 we deduce the following conservation of the total mass current:

$$
\left\{\operatorname{div}\left(j_{M}^{0}, j_{M}^{1}, j_{M}^{2}, j_{M}^{3}\right)\right\}_{K}\left(x^{0}, x^{1}, x^{2}, x^{3}\right)=0 \quad \forall\left(x^{0}, x^{1}, x^{2}, x^{3}\right) \in \mathbb{R}^{4},
$$

where the covariant divergence $\{\operatorname{div}(\cdot)\}_{K}$ is defined by (3.7). Furthermore, define the total scalar density $\mathcal{L}_{M}:=\mathcal{L}_{M}\left(\left(\left\{K_{m n}\right\}_{m, n=0,1,2,3}\right),\left(x^{0}, x^{1}, x^{2}, x^{3}\right)\right)$ of the given Lagrangian of the system of 
particles by:

$$
\mathcal{L}_{M}\left(\left(\left\{K_{m n}\right\}_{m, n=0,1,2,3}\right),\left(x^{0}, x^{1}, x^{2}, x^{3}\right)\right):=\sum_{k=1}^{N} \rho_{W_{L_{\mathcal{G}}, k}}\left(x^{0}, x^{1}, x^{2}, x^{3}\right) \quad \forall\left(x^{0}, x^{1}, x^{2}, x^{3}\right) \in \mathbb{R}^{4},
$$

where $W_{L_{\mathcal{G}}, k}\left(s_{k}\right)$ is given by (3.12), so that

$$
\begin{array}{r}
\mathcal{L}_{M}\left(\left(\left\{K_{m n}\right\}_{m, n=0,1,2,3}\right),\left(x^{0}, x^{1}, x^{2}, x^{3}\right)\right):=\sum_{k=1}^{N} \int_{a_{k}}^{b_{k}}\{ \\
\left.\left|\operatorname{det}\left\{K^{p q}\left(\chi_{k}\left(s_{k}\right)\right)\right\}\right|^{\frac{1}{2}} W_{L_{\mathcal{G}}, k}\left(s_{k}\right)\left(\sum_{j=0}^{3} w_{j}\left(\chi_{k}\left(s_{k}\right)\right) \frac{d \chi_{k}^{j}}{d s_{k}}\left(s_{k}\right)\right) \delta\left(x^{0}-\chi_{k}^{0}\left(s_{k}\right), \ldots, x^{3}-\chi_{k}^{3}\left(s_{k}\right)\right)\right\} d s_{k} \\
\forall\left(x^{0}, x^{1}, x^{2}, x^{3}\right) \in \mathbb{R}^{4} .
\end{array}
$$

In particular, by (3.24) and (3.12) we have

$$
\begin{gathered}
\iiint \int_{\mathbb{R}^{4}} \mathcal{L}_{M}\left(\left(\left\{K_{m n}\right\}_{m, n=0,1,2,3}\right),\left(x^{0}, x^{1}, x^{2}, x^{3}\right)\right)\left|\operatorname{det}\left\{K^{p q}\left(\left(x^{0}, x^{1}, x^{2}, x^{3}\right)\right)\right\}\right|^{-\frac{1}{2}} d x^{0} d x^{1} d x^{2} d x^{3} \\
=\sum_{k=1}^{N} \int_{a_{k}}^{b_{k}} W_{L_{\mathcal{G}}, k}\left(s_{k}\right)\left(\sum_{j=0}^{3} w_{j}\left(\chi_{k}\left(s_{k}\right)\right) \frac{d \chi_{k}^{j}}{d s_{k}}\left(s_{k}\right)\right) d s_{k}= \\
\sum_{k=1}^{N} \int_{a_{k}}^{b_{k}}\left\{-m_{k} \mathcal{G}\left(\sum_{j=0}^{3} \sum_{m=0}^{3} K_{j m}\left(\chi_{k}\left(s_{k}\right)\right) u_{k}^{j}\left(s_{k}\right) u_{k}^{m}\left(s_{k}\right)\right)\right\}\left(\sum_{j=0}^{3} w_{j}\left(\chi_{k}\left(s_{k}\right)\right) \frac{d \chi_{k}^{j}}{d s_{k}}\left(s_{k}\right)\right) d s_{k} \\
+\sum_{k=1}^{N} \int_{a_{k}}^{b_{k}}\left\{-\sum_{j=0}^{3} \sigma_{k} u_{k}^{j}(s) A_{j}\left(\chi_{k}\left(s_{k}\right)\right)-U_{k}\left(\chi_{k}\left(s_{k}\right)\right)\right\}\left(\sum_{j=0}^{3} w_{j}\left(\chi_{k}\left(s_{k}\right)\right) \frac{d \chi_{k}^{j}}{d s_{k}}\left(s_{k}\right)\right) d s_{k},
\end{gathered}
$$

so that

$$
\begin{array}{r}
\iiint_{\mathbb{R}^{4}} \mathcal{L}_{M}\left(\left(\left\{K_{m n}\right\}_{m, n=0,1,2,3}\right),\left(x^{0}, x^{1}, x^{2}, x^{3}\right)\right)\left|\operatorname{det}\left\{K^{p q}\left(\left(x^{0}, x^{1}, x^{2}, x^{3}\right)\right)\right\}\right|^{-\frac{1}{2}} d x^{0} d x^{1} d x^{2} d x^{3} \\
=\sum_{k=1}^{N} L_{\mathcal{G}, k}\left(\chi_{k}\right), \quad(3.26)
\end{array}
$$

where, as before in (2.100) we denote the Lagrangian of the $k$-th particle as

$$
\begin{aligned}
L_{\mathcal{G}, k}\left(\chi_{k}\right)=\int_{a_{k}}^{b_{k}}\left\{-m_{k} \mathcal{G}\left(\sum_{j=0}^{3} \sum_{m=0}^{3} K_{j m}\left(\chi_{k}\left(s_{k}\right)\right) u_{k}^{j}(s) u_{k}^{m}\left(s_{k}\right)\right)\right\}\left(\sum_{j=0}^{3} w_{j}\left(\chi_{k}\left(s_{k}\right)\right) \frac{d \chi_{k}^{j}}{d s_{k}}\right) d s_{k} \\
+\int_{a_{k}}^{b_{k}}\left\{-\sum_{j=0}^{3} \sigma_{k} u_{k}^{j}\left(s_{k}\right) A_{j}\left(\chi_{k}\left(s_{k}\right)\right)\right\}\left(\sum_{j=0}^{3} w_{j}\left(\chi_{k}\left(s_{k}\right)\right) \frac{d \chi_{k}^{j}}{d s_{k}}\right) d s_{k} \\
=\int_{a_{k}}^{b_{k}}\left\{-m_{k} \mathcal{G}\left(\sum_{j=0}^{3} \sum_{m=0}^{3} K_{j m}\left(\chi_{k}\left(s_{k}\right)\right) u_{k}^{j}(s) u_{k}^{m}\left(s_{k}\right)\right)\right\}\left(\sum_{j=0}^{3} w_{j}\left(\chi_{k}\left(s_{k}\right)\right) \frac{d \chi_{k}^{j}}{d s_{k}}\right) d s_{k} \\
+\int_{a_{k}}^{b_{k}}\left\{-\sum_{j=0}^{3} \sigma_{k} A_{j}\left(\chi_{k}\left(s_{k}\right)\right) \frac{d \chi_{k}^{j}}{d s_{k}}\right\} d s_{k} \quad \forall k=1,2, \ldots N .
\end{aligned}
$$


Moreover, define the total scalar residual density $\hat{\mathcal{L}}_{M}:=\hat{\mathcal{L}}_{M}\left(\left(\left\{K_{m n}\right\}_{m, n=0,1,2,3}\right),\left(x^{0}, x^{1}, x^{2}, x^{3}\right)\right)$ of the given Lagrangian of the system of particles by:

$$
\hat{\mathcal{L}}_{M}\left(\left(\left\{K_{m n}\right\}_{m, n=0,1,2,3}\right),\left(x^{0}, x^{1}, x^{2}, x^{3}\right)\right):=\sum_{k=1}^{N} \rho_{\hat{W}_{L_{\mathcal{G}}, k}}\left(x^{0}, x^{1}, x^{2}, x^{3}\right) \quad \forall\left(x^{0}, x^{1}, x^{2}, x^{3}\right) \in \mathbb{R}^{4},
$$

where $\hat{W}_{L_{\mathcal{G}}, k}\left(s_{k}\right)$ is given by $(3.14)$, so that

$$
\begin{array}{r}
\hat{\mathcal{L}}_{M}\left(\left(\left\{K_{m n}\right\}_{m, n=0,1,2,3}\right),\left(x^{0}, x^{1}, x^{2}, x^{3}\right)\right):=\sum_{k=1}^{N} \int_{a_{k}}^{b_{k}}\{ \\
\left.\left|\operatorname{det}\left\{K^{p q}\left(\chi_{k}\left(s_{k}\right)\right)\right\}\right|^{\frac{1}{2}} \hat{W}_{L_{\mathcal{G}}, k}\left(s_{k}\right)\left(\sum_{j=0}^{3} w_{j}\left(\chi_{k}\left(s_{k}\right)\right) \frac{d \chi_{k}^{j}}{d s_{k}}\left(s_{k}\right)\right) \delta\left(x^{0}-\chi_{k}^{0}\left(s_{k}\right), \ldots, x^{3}-\chi_{k}^{3}\left(s_{k}\right)\right)\right\} d s_{k} \\
\forall\left(x^{0}, x^{1}, x^{2}, x^{3}\right) \in \mathbb{R}^{4} .
\end{array}
$$

Then, by (3.16), (3.24), (3.29), (3.12) and (3.14) together we deduce:

$$
\begin{aligned}
\mathcal{L}_{M}\left(\left(\left\{K_{m n}\right\}_{m, n=0,1,2,3}\right),\left(x^{0}, x^{1}, x^{2}, x^{3}\right)\right)=\hat{\mathcal{L}}_{M}\left(\left(\left\{K_{m n}\right\}_{m, n=0,1,2,3}\right),\left(x^{0}, x^{1}, x^{2}, x^{3}\right)\right) \\
-\left(\sum_{k=0}^{3} A_{k}\left(x^{0}, x^{1}, x^{2}, x^{3}\right) j^{k}\left(x^{0}, x^{1}, x^{2}, x^{3}\right)\right) \quad \forall\left(x^{0}, x^{1}, x^{2}, x^{3}\right) \in \mathbb{R}^{4} .
\end{aligned}
$$

\section{The total simplified Lagrangian in (2.219), (2.220), for the limiting case of (2.216) in a cartesian coordinate system}

Again consider the strongly-correlated kinematical contravariant pseudo-metric $\left\{J^{m n}\right\}_{m, n=0,1,2,3}$ and the kinematical global time $\varphi$, forming a standard kinematical Lorentz's structure with global time on $\mathbb{R}^{4}$, as in Proposition 2.7 and Remark 2.6. Furthermore, let $\left\{J_{m n}\right\}_{m, n=0,1,2,3}$ be the inverse covariant pseudo-metric on $\mathbb{R}^{4}$, associated with $\left\{J^{m n}\right\}_{m, n=0,1,2,3}$, which satisfies

$$
\sum_{k=0}^{3} J^{m k} J_{k n}=\left\{\begin{array}{lll}
1 & \text { if } & m=n \\
0 & \text { if } & m \neq n
\end{array} \quad \forall m, n=0,1,2,3 .\right.
$$

We consider the Lagrangian in (2.219), (2.220), corresponding to the limiting case of (2.216), where as before we have

$$
\begin{gathered}
\left(w_{0}, w_{1}, w_{2}, w_{3}\right)=\left(\frac{\partial \varphi}{\partial x^{0}}, \frac{\partial \varphi}{\partial x^{1}}, \frac{\partial \varphi}{\partial x^{2}}, \frac{\partial \varphi}{\partial x^{3}}\right) \quad \forall\left(x^{0}, x^{1}, x^{2}, x^{3}\right) \in \mathbb{R}^{4},, \\
\sum_{m=0}^{3} \sum_{n=0}^{3} J^{m n} \frac{\partial \varphi}{\partial x^{m}} \frac{\partial \varphi}{\partial x^{n}}=1 \quad \forall\left(x^{0}, x^{1}, x^{2}, x^{3}\right) \in \mathbb{R}^{4},
\end{gathered}
$$

the dynamical global time equals to global kinematical time $\varphi$ and the dynamical tensor of threedimensional Geometry $\left\{\Lambda^{m n}\right\}_{m, n=0,1,2,3}$ equals to the kinematical tensor of three-dimensional Ge- 
ometry $\left\{\Theta^{m n}\right\}_{m, n=0,1,2,3}$, so that

$$
\Lambda^{m n}=\Theta^{m n}:=r^{m} r^{n}-J^{m n}=\left(\sum_{j=0}^{3} J^{m j} \frac{\partial \varphi}{\partial x^{j}}\right)\left(\sum_{j=0}^{3} J^{n j} \frac{\partial \varphi}{\partial x^{j}}\right)-J^{m n} \quad \forall m, n=0,1,2,3,
$$

where $\left(r^{0}, r^{1}, r^{2}, r^{3}\right)$ is the contravariant four-vector of the inertia, corresponding to $\left\{J^{m n}\right\}_{m, n=0,1,2,3}$ and $\varphi$, defined by

$$
r^{m}:=\sum_{j=0}^{3} J^{m j} \frac{\partial \varphi}{\partial x^{j}} \quad \forall m=0,1,2,3 .
$$

Note here that by (4.5) and (4.3) we have

$$
\sum_{j=0}^{3} r^{j} \frac{\partial \varphi}{\partial x^{j}}=1 \quad \forall\left(x^{0}, x^{1}, x^{2}, x^{3}\right) \in \mathbb{R}^{4} .
$$

Next, as before, given, a four-covector field of genuine gravity $\left(S_{0}, S_{1}, S_{2}, S_{3}\right)$ we define the dynamical pseudometric $\left\{K^{m n}\right\}_{m, n=0,1,2,3}$ by the formula

$$
\sum_{k=0}^{3} K^{m k} K_{k n}=\left\{\begin{array}{lll}
1 & \text { if } & m=n \\
0 & \text { if } & m \neq n
\end{array} \quad \forall m, n=0,1,2,3\right.
$$

with

$$
K_{j m}:=\left(J_{j m}+S_{m} \frac{\partial \varphi}{\partial x^{j}}+\frac{\partial \varphi}{\partial x^{m}} S_{j}\right) \quad \forall 0 \leq j, m \leq 3 \quad \forall\left(x^{0}, x^{1}, x^{2}, x^{3}\right) \in \mathbb{R}^{4},
$$

where we assume that the four-covector field of genuine gravity $\left(S_{0}, S_{1}, S_{2}, S_{3}\right)$ satisfies the calibration:

$$
\operatorname{det}\left(\left\{K_{m n}\right\}_{m, n=0,1,2,3}\right)=\operatorname{det}\left(\left\{J_{m n}\right\}_{m, n=0,1,2,3}\right) \quad \forall\left(x^{0}, x^{1}, x^{2}, x^{3}\right) \in \mathbb{R}^{4},
$$

so that $\left(S_{0}, S_{1}, S_{2}, S_{3}\right)$ has only three independent components. Moreover, in the latter case we have

$$
\sum_{m=0}^{3} \sum_{n=0}^{3} K^{m n} \frac{\partial \varphi}{\partial x^{m}} \frac{\partial \varphi}{\partial x^{n}}=\sum_{m=0}^{3} \sum_{n=0}^{3} J^{m n} \frac{\partial \varphi}{\partial x^{m}} \frac{\partial \varphi}{\partial x^{n}}=1 \quad \forall\left(x^{0}, x^{1}, x^{2}, x^{3}\right) \in \mathbb{R}^{4},
$$

and both couples $\left\{J^{m n}\right\}_{m, n=0,1,2,3}, \varphi$, and $\left\{K^{m n}\right\}_{m, n=0,1,2,3}, \varphi$ are strongly correlated, so that, $\left\{K^{m n}\right\}_{m, n=0,1,2,3}$, with global time $\varphi$, is a kinematically semi-scalar super-correlated pseudometric with with global time $\varphi$, corresponding to the covector of genuine gravity $\left(S_{0}, S_{1}, S_{2}, S_{3}\right)$. Moreover, as before, we have

$$
K^{m n}=v^{m} v^{n}-\Lambda^{m n}=J^{m n}+v^{m} v^{n}-r^{m} r^{n} \quad \forall m, n=0,1,2,3,
$$

where the contravariant vector of generalized gravity $\left(v^{0}, v^{1}, v^{2}, v^{3}\right)$ is defined by the following covariant relation:

$$
v^{m}:=\left(\sum_{j=0}^{3} \Lambda^{m j} S_{j}\right)+r^{m} \quad \forall m=0,1,2,3,
$$

and $\left(r^{0}, r^{1}, r^{2}, r^{3}\right)$ is given by (4.5). Moreover, the following covariant relations are valid

$$
\left(\sum_{j=0}^{3} v^{j} \frac{\partial \varphi}{\partial x^{j}}\right)=1
$$




$$
\begin{gathered}
S_{m}:=\frac{1}{2}\left(\sum_{j=0}^{3} K_{m j} r^{j}\right)-\frac{1}{2}\left(\sum_{j=0}^{3} J_{m j} v^{j}\right) \quad \forall 0 \leq m \leq 3, \\
\sum_{j=0}^{3} \sum_{m=0}^{3} K_{j m} v^{j} v^{m}=1,
\end{gathered}
$$

and

$$
\sum_{j=0}^{3} K^{j m} \frac{\partial \varphi}{\partial x^{j}}=v^{m} \quad \forall m=0,1,2,3 .
$$

Finally, as before, given arbitrary contravariant vector $\left(v^{0}, v^{1}, v^{2}, v^{3}\right)$, satisfying

$$
\left(\sum_{j=0}^{3} v^{j} \frac{\partial \varphi}{\partial x^{j}}\right)=1
$$

as in (4.13) (we call such a four-vector speed-like four-vector), if we define $\left\{K^{m n}\right\}_{m, n=0,1,2,3}$, as in (4.11), by

$$
K^{m n}=v^{m} v^{n}-\Lambda_{m n}=J^{m n}+v^{m} v^{n}-r^{m} r^{n} \quad \forall m, n=0,1,2,3,
$$

then the inverse tensor, $\left\{K_{m n}\right\}_{m, n=0,1,2,3}$, given by

$$
\sum_{k=0}^{3} K^{m k} K_{k n}=\left\{\begin{array}{lll}
1 & \text { if } & m=n \\
0 & \text { if } & m \neq n
\end{array} \quad \forall m, n=0,1,2,3\right.
$$

necessary satisfies

$$
K_{j m}:=\left(J_{j m}+S_{m} \frac{\partial \varphi}{\partial x^{j}}+\frac{\partial \varphi}{\partial x^{m}} S_{j}\right) \quad \forall 0 \leq j, m \leq 3 \quad \forall\left(x^{0}, x^{1}, x^{2}, x^{3}\right) \in \mathbb{R}^{4},
$$

and

$$
\operatorname{det}\left(\left\{K_{m n}\right\}_{m, n=0,1,2,3}\right)=\operatorname{det}\left(\left\{J_{m n}\right\}_{m, n=0,1,2,3}\right) \quad \forall\left(x^{0}, x^{1}, x^{2}, x^{3}\right) \in \mathbb{R}^{4},
$$

as in (4.8) and (4.9), where $\left(S_{0}, S_{1}, S_{2}, S_{3}\right)$ satisfies

$$
S_{m}:=\frac{1}{2}\left(\sum_{j=0}^{3} K_{m j} r^{j}\right)-\frac{1}{2}\left(\sum_{j=0}^{3} J_{m j} v^{j}\right) \quad \forall 0 \leq m \leq 3,
$$

and

$$
v^{m}:=\left(\sum_{j=0}^{3} \Lambda^{m j} S_{j}\right)+r^{m} \quad \forall m=0,1,2,3,
$$

as in (4.14), and (4.12). So we have a one-to-one and onto mappings between contravariant fourvectors, $\left(v^{0}, v^{1}, v^{2}, v^{3}\right)$, satisfying the restriction (4.17), and four covectors $\left(S_{0}, S_{1}, S_{2}, S_{3}\right)$, satisfying the restriction (4.21), where $\left\{K_{m n}\right\}_{m, n=0,1,2,3}$ is given by (4.20). So the speed-like contravariant four-vectors of generalized gravity $\left(v^{0}, v^{1}, v^{2}, v^{3}\right)$, satisfying the restriction (4.17), can be considered as an independent argument in the simplified Lagrangian in (2.219), (2.220), instead of the fourcovector of genuine gravity $\left(S_{0}, S_{1}, S_{2}, S_{3}\right)$, satisfying the restriction (4.21). 
Next, assume that we fix some cartesian coordinate system, so that in this system we have:

$$
\left(w_{0}, w_{1}, w_{2}, w_{3}\right)=\left(\frac{\partial \varphi}{\partial x^{0}}, \frac{\partial \varphi}{\partial x^{1}}, \frac{\partial \varphi}{\partial x^{2}}, \frac{\partial \varphi}{\partial x^{3}}\right)=(1,0,0,0) \quad \forall\left(x^{0}, x^{1}, x^{2}, x^{3}\right) \in \mathbb{R}^{4},
$$

and

$$
\left\{\begin{array}{l}
\Lambda^{00}=\Theta^{00}=0 \\
\Lambda^{0 j}=\Lambda^{j 0}=\Theta^{0 j}=\Theta^{j 0}=0 \quad \forall j=1,2,3 \\
\Lambda^{j m}=\Theta^{j m}=\delta_{j m} \quad \forall j, m=1,2,3 .
\end{array}\right.
$$

In particular, (4.17) reeds:

$$
v^{0}=1
$$

so that

$$
\left(v^{0}, v^{1}, v^{2}, v^{3}\right)=\left(1, v^{1}, v^{2}, v^{3}\right)=(1, \mathbf{v}) \quad \text { where } \quad \mathbf{v}:=\left(v^{1}, v^{2}, v^{3}\right) \in \mathbb{R}^{3} .
$$

Therefore, the independent argument of the Lagrangian is actually a three-dimensional vector field $\mathbf{v}$. We call $\mathbf{v}$ the three-dimensional gravitational vector potential, however, note that it defined only in cartesian coordinate systems. Similarly, by (4.6) we have

$$
\left(r^{0}, r^{1}, r^{2}, r^{3}\right)=\left(1, r^{1}, r^{2}, r^{3}\right)=(1, \mathbf{r}) \quad \text { where } \quad \mathbf{r}:=\left(r^{1}, r^{2}, r^{3}\right) \in \mathbb{R}^{3} .
$$

We call $\mathbf{r}$ the three-dimensional vector potential of inertia, and as before, note that it defined only in cartesian coordinate systems. Furthermore, by (4.18), (4.25) and (4.27) we deduce:

$$
\left\{\begin{array}{l}
K^{00}=1 \\
K^{j m}=-\delta_{j m}+v^{j} v^{m} \quad \forall 1 \leq j, m \leq 3 \\
K^{0 j}=K^{j 0}=v^{j} \quad \forall 1 \leq j \leq 3,
\end{array}\right.
$$

and therefore, as before, we have

$$
\left\{\begin{array}{l}
K_{00}=1-|\mathbf{v}|^{2} \\
K_{j m}=-\delta_{j m} \quad \forall 1 \leq j, m \leq 3 \\
K_{0 j}=K_{j 0}=v^{j} \quad \forall 1 \leq j \leq 3
\end{array}\right.
$$

and

$$
\operatorname{det}\left(\left\{K_{m n}\right\}_{m, n=0,1,2,3}\right)=\operatorname{det}\left(\left\{J_{m n}\right\}_{m, n=0,1,2,3}\right)=1 \quad \forall\left(x^{0}, x^{1}, x^{2}, x^{3}\right) \in \mathbb{R}^{4} .
$$

Similarly,

$$
\left\{\begin{array}{l}
J^{00}=1 \\
J^{j m}=-\delta_{j m}+r^{j} r^{m} \quad \forall 1 \leq j, m \leq 3 \\
J^{0 j}=J^{j 0}=r^{j} \quad \forall 1 \leq j \leq 3,
\end{array}\right.
$$


and therefore, by Lemma 11.5 from the Appendix we have

$$
\left\{\begin{array}{l}
J_{00}=1-|\mathbf{r}|^{2} \\
J_{j m}=-\delta_{j m} \quad \forall 1 \leq j, m \leq 3 \\
J_{0 j}=J_{j 0}=r^{j} \quad \forall 1 \leq j \leq 3 .
\end{array}\right.
$$

On the other hand, by (4.33), (4.24) identity (4.20) reads as

$$
\left\{\begin{array}{l}
K_{00}=1-|\mathbf{r}|^{2}+2 S_{0} \\
K_{j m}=-\delta_{j m} \quad \forall 1 \leq j, m \leq 3 \\
K_{0 j}=K_{j 0}=S_{j}+r^{j} \quad \forall 1 \leq j \leq 3,
\end{array}\right.
$$

and thus, comparing (4.30) with (4.34) we deduce:

$$
\left\{\begin{array}{l}
1-|\mathbf{v}|^{2}=1-|\mathbf{r}|^{2}+2 S_{0} \\
v^{j}=S_{j}+r^{j} \quad \forall 1 \leq j \leq 3,
\end{array}\right.
$$

so that,

$$
\begin{cases}S_{0}:=\frac{1}{2}|\mathbf{r}|^{2}-\frac{1}{2}|\mathbf{v}|^{2} & \\ S_{j}:=v^{j}-r^{j} & \forall 1 \leq j \leq 3 .\end{cases}
$$

Furthermore, since $\varphi=x^{0}+$ Const, the first coordinate can be chosen as the proper parameter $s_{k}$ on the Lagrangian of every particle, so that we rewrite (2.207) as

$$
L_{M}\left(\left\{K_{m n}\right\}_{m, n=0,1,2,3}\right):=\iiint \int_{\mathcal{V}}\left(\mathcal{L}_{M}\left(\left(\left\{K_{m n}\right\}_{m, n=0,1,2,3}\right),\left(x^{0}, x^{1}, x^{2}, x^{3}\right)\right)\right) d x^{0} d x^{1} d x^{2} d x^{3}
$$

and (2.209) as:

$$
\begin{aligned}
& \mathcal{L}_{M}\left(\left(\left\{K_{m n}\right\}_{m, n=0,1,2,3}\right),\left(x^{0}, x^{1}, x^{2}, x^{3}\right)\right):=\sum_{k=1}^{N} \int_{-\infty}^{+\infty}\{ \\
& \left.W_{L_{\mathcal{G}}, k}\left(\left(\chi^{0}\right)\right)\left(\sum_{j=0}^{3} w_{j}\left(\chi_{k}\left(\chi^{0}\right)\right) \frac{d \chi_{k}^{j}}{d \chi^{0}}\left(\chi^{0}\right)\right) \delta\left(x^{0}-\chi^{0}, x^{1}-\chi_{k}^{1}\left(\chi^{0}\right), x^{2}-\chi_{k}^{2}\left(\chi^{0}\right), x^{3}-\chi_{k}^{3}\left(\chi^{0}\right)\right)\right\} d \chi^{0} \\
& =\sum_{k=1}^{N} W_{L_{\mathcal{G}}, k}\left(x^{0}\right) \delta\left(x^{1}-\chi_{k}^{1}\left(\chi^{0}\right), x^{2}-\chi_{k}^{2}\left(\chi^{0}\right), x^{3}-\chi_{k}^{3}\left(\chi^{0}\right)\right) \quad \forall\left(x^{0}, x^{1}, x^{2}, x^{3}\right) \in \mathbb{R}^{4}, \quad(4.38)
\end{aligned}
$$

where the $\delta$-function in the last expression is three-dimensional, $W_{L_{\mathcal{G}}, k}\left(x^{0}\right)$ is given, as in $(3.12)$, by

$$
\begin{array}{r}
W_{L_{\mathcal{G}}, k}\left(x^{0}\right):=-m_{k} \mathcal{G}\left(\sum_{j=0}^{3} \sum_{m=0}^{3} K_{j m}\left(\chi_{k}\left(x^{0}\right)\right) \frac{d \chi_{k}^{j}}{d x^{0}}\left(x^{0}\right) \frac{d \chi_{k}^{m}}{d x^{0}}\left(x^{0}\right)\right)-\sum_{j=0}^{3} \sigma_{k} \frac{d \chi_{k}^{j}}{d x^{0}}\left(x^{0}\right) A_{j}\left(\chi_{k}\left(x^{0}\right)\right) \\
\forall s \in(-\infty,+\infty) \quad \forall k=1,2, \ldots, N, \quad(4.39)
\end{array}
$$


and the four-dimensional trajectory of the $k$-th particle, parameterized by the firs coordinate $x^{0}$ is given by

$$
\left\{\begin{array}{lr}
\chi_{k}\left(x^{0}\right):=\left(\chi_{k}^{0}\left(x^{0}\right), \chi_{k}^{1}\left(x^{0}\right), \chi_{k}^{2}\left(x^{0}\right), \chi_{k}^{3}\left(x^{0}\right)\right):=\left(x^{0}, \chi_{k}^{1}\left(x^{0}\right), \chi_{k}^{2}\left(x^{0}\right), \chi_{k}^{3}\left(x^{0}\right)\right) & \forall k=1,2, \ldots N \\
\left(\frac{d \chi_{k}^{0}}{d x^{0}}\left(x^{0}\right), \frac{d \chi_{k}^{1}}{d x^{0}}\left(x^{0}\right), \frac{d \chi_{k}^{2}}{d x^{0}}\left(x^{0}\right), \frac{d \chi_{k}^{3}}{d x^{0}}\left(x^{0}\right)\right):=\left(1, \frac{d \chi_{k}^{1}}{d x^{0}}\left(x^{0}\right), \frac{d \chi_{k}^{2}}{d x^{0}}\left(x^{0}\right), \frac{d \chi_{k}^{3}}{d x^{0}}\left(x^{0}\right)\right) \quad \forall k=1,2, \ldots N .
\end{array}\right.
$$

Similarly, the four-current and the charge densities satisfy:

$$
\begin{array}{r}
\left(j^{0}, j^{1}, j^{2}, j^{3}\right)=\sum_{k=1}^{N} \sigma_{k}\left(1, \frac{d \chi_{k}^{1}}{d x^{0}}\left(x^{0}\right), \frac{d \chi_{k}^{2}}{d x^{0}}\left(x^{0}\right), \frac{d \chi_{k}^{3}}{d x^{0}}\left(x^{0}\right)\right) \delta\left(x^{1}-\chi_{k}^{1}\left(\chi^{0}\right), x^{2}-\chi_{k}^{2}\left(\chi^{0}\right), x^{3}-\chi_{k}^{3}\left(\chi^{0}\right)\right) \\
\forall\left(x^{0}, x^{1}, x^{2}, x^{3}\right) \in \mathbb{R}^{4}, \quad(4.41)
\end{array}
$$

and

$$
\rho=\sum_{k=1}^{N} \sigma_{k} \delta\left(x^{1}-\chi_{k}^{1}\left(\chi^{0}\right), x^{2}-\chi_{k}^{2}\left(\chi^{0}\right), x^{3}-\chi_{k}^{3}\left(\chi^{0}\right)\right) \quad \forall\left(x^{0}, x^{1}, x^{2}, x^{3}\right) \in \mathbb{R}^{4} .
$$

In particular, we have

$$
\left(j^{0}, j^{1}, j^{2}, j^{3}\right)=(\rho, \mathbf{j}) \quad \text { where } \quad \mathbf{j}:=\left(j^{1}, j^{2}, j^{3}\right) \in \mathbb{R}^{3} \quad \forall\left(x^{0}, x^{1}, x^{2}, x^{3}\right) \in \mathbb{R}^{4},
$$

where

$$
\begin{array}{r}
\mathbf{j}:=\left(j^{1}, j^{2}, j^{3}\right)=\sum_{k=1}^{N} \sigma_{k}\left(\frac{d \chi_{k}^{1}}{d x^{0}}\left(x^{0}\right), \frac{d \chi_{k}^{2}}{d x^{0}}\left(x^{0}\right), \frac{d \chi_{k}^{3}}{d x^{0}}\left(x^{0}\right)\right) \delta\left(x^{1}-\chi_{k}^{1}\left(\chi^{0}\right), x^{2}-\chi_{k}^{2}\left(\chi^{0}\right), x^{3}-\chi_{k}^{3}\left(\chi^{0}\right)\right) \\
\forall\left(x^{0}, x^{1}, x^{2}, x^{3}\right) \in \mathbb{R}^{4} .
\end{array}
$$

In the same way, the mass four-current and the mass densities satisfy:

$$
\begin{array}{r}
\left(j_{M}^{0}, j_{M}^{1}, j_{M}^{2}, j_{M}^{3}\right)= \\
\sum_{k=1}^{N} m_{k}\left(1, \frac{d \chi_{k}^{1}}{d x^{0}}\left(x^{0}\right), \frac{d \chi_{k}^{2}}{d x^{0}}\left(x^{0}\right), \frac{d \chi_{k}^{3}}{d x^{0}}\left(x^{0}\right)\right) \delta\left(x^{1}-\chi_{k}^{1}\left(\chi^{0}\right), x^{2}-\chi_{k}^{2}\left(\chi^{0}\right), x^{3}-\chi_{k}^{3}\left(\chi^{0}\right)\right) \\
\forall\left(x^{0}, x^{1}, x^{2}, x^{3}\right) \in \mathbb{R}^{4},
\end{array}
$$

and

$$
M=\sum_{k=1}^{N} m_{k} \delta\left(x^{1}-\chi_{k}^{1}\left(\chi^{0}\right), x^{2}-\chi_{k}^{2}\left(\chi^{0}\right), x^{3}-\chi_{k}^{3}\left(\chi^{0}\right)\right) \quad \forall\left(x^{0}, x^{1}, x^{2}, x^{3}\right) \in \mathbb{R}^{4} .
$$

In particular, we have

$$
\left(j_{M}^{0}, j_{M}^{1}, j_{M}^{2}, j_{M}^{3}\right)=\left(M, \mathbf{j}_{M}\right) \quad \text { where } \quad \mathbf{j}_{M}:=\left(j_{M}^{1}, j_{M}^{2}, j_{M}^{3}\right) \in \mathbb{R}^{3} \quad \forall\left(x^{0}, x^{1}, x^{2}, x^{3}\right) \in \mathbb{R}^{4},
$$


where

$$
\begin{aligned}
& \mathbf{j}_{M}:=\left(j_{M}^{1}, j_{M}^{2}, j_{M}^{3}\right)= \\
& \sum_{k=1}^{N} m_{k}\left(\frac{d \chi_{k}^{1}}{d x^{0}}\left(x^{0}\right), \frac{d \chi_{k}^{2}}{d x^{0}}\left(x^{0}\right), \frac{d \chi_{k}^{3}}{d x^{0}}\left(x^{0}\right)\right) \delta\left(x^{1}-\chi_{k}^{1}\left(\chi^{0}\right), x^{2}-\chi_{k}^{2}\left(\chi^{0}\right), x^{3}-\chi_{k}^{3}\left(\chi^{0}\right)\right) \\
& \forall\left(x^{0}, x^{1}, x^{2}, x^{3}\right) \in \mathbb{R}^{4} .
\end{aligned}
$$

Note that by (3.18), (3.22), using (4.31), (4.43) and (4.47) we have the conservation of the charge and the mass current:

$$
\begin{array}{cc}
\frac{\partial \rho}{\partial x^{0}}+\operatorname{div}_{\mathbf{x}} \mathbf{j}=0 & \forall\left(x^{0}, x^{1}, x^{2}, x^{3}\right) \in \mathbb{R}^{4}, \\
\frac{\partial M}{\partial x^{0}}+\operatorname{div}_{\mathbf{x}} \mathbf{j}_{M}=0 & \forall\left(x^{0}, x^{1}, x^{2}, x^{3}\right) \in \mathbb{R}^{4} .
\end{array}
$$

Moreover, by (4.39), (4.30) and (4.40) we have:

$$
\begin{array}{r}
W_{L_{\mathcal{G}}, k}\left(x^{0}\right)=-m_{k} \mathcal{G}\left(1-\sum_{j=1}^{3}\left(\frac{d \chi_{k}^{j}}{d x^{0}}\left(x^{0}\right)-v^{j}\left(\chi_{k}\left(x^{0}\right)\right)\right)^{2}\right)-\sum_{j=0}^{3} \sigma_{k} \frac{d \chi_{k}^{j}}{d x^{0}}\left(x^{0}\right) A_{j}\left(\chi_{k}\left(x^{0}\right)\right) \\
\forall s \in(-\infty,+\infty) \quad \forall k=1,2, \ldots, N,
\end{array}
$$

so that, by (4.51) and (4.41) we rewrite (4.38) as:

$$
\begin{array}{r}
\mathcal{L}_{M}\left(\left(\left\{K_{m n}\right\}_{m, n=0,1,2,3}\right),\left(x^{0}, x^{1}, x^{2}, x^{3}\right)\right):=-\sum_{n=0}^{3} A_{n}\left(x^{0}, x^{1}, x^{2}, x^{3}\right) j^{n}\left(x^{0}, x^{1}, x^{2}, x^{3}\right) \\
-\sum_{k=1}^{N} m_{k} \mathcal{G}\left(1-\sum_{j=1}^{3}\left(\frac{d \chi_{k}^{j}}{d x^{0}}\left(x^{0}\right)-v^{j}\left(\chi_{k}\left(x^{0}\right)\right)\right)^{2}\right) \delta\left(x^{1}-\chi_{k}^{1}\left(\chi^{0}\right), x^{2}-\chi_{k}^{2}\left(\chi^{0}\right), x^{3}-\chi_{k}^{3}\left(\chi^{0}\right)\right) \\
\forall\left(x^{0}, x^{1}, x^{2}, x^{3}\right) \in \mathbb{R}^{4} .
\end{array}
$$

Then, by (4.52) and (4.37), in the case $\mathcal{V}=\mathbb{R}^{4}$ we deduce

$$
\begin{aligned}
L_{M}\left(\left\{K_{m n}\right\}_{m, n=0,1,2,3}\right) & =-\sum_{k=1}^{N} m_{k} \int_{-\infty}^{+\infty} \mathcal{G}\left(1-\sum_{j=1}^{3}\left(\frac{d \chi_{k}^{j}}{d x^{0}}\left(x^{0}\right)-v^{j}\left(\chi_{k}\left(x^{0}\right)\right)\right)^{2}\right) d x^{0} \\
& -\iiint \int_{\mathbb{R}^{4}}\left(\sum_{n=0}^{3} A_{n}\left(x^{0}, x^{1}, x^{2}, x^{3}\right) j^{n}\left(x^{0}, x^{1}, x^{2}, x^{3}\right)\right) d x^{0} d x^{1} d x^{2} d x^{3} .
\end{aligned}
$$

Next we denote

$$
\left(A_{0}, A_{1}, A_{2}, A_{3}\right)=(\Psi,-\mathbf{A}) \quad \text { where } A_{0}=\Psi \text { and }\left(A_{1}, A_{2}, A_{3}\right)=-\mathbf{A} \text {, }
$$

and

$$
\left(S_{0}, S_{1}, S_{2}, S_{3}\right):=-(\Phi,-\mathbf{h}) \quad \text { where } \quad S_{0}=-\Phi \quad \text { and } \quad\left(S_{1}, S_{2}, S_{3}\right)=\mathbf{h} \text {. }
$$


In particular, by $(4.36)$ we have:

$$
\left\{\begin{array}{l}
\Phi=\frac{1}{2}\left(|\mathbf{v}|^{2}-|\mathbf{r}|^{2}\right) \\
\mathbf{h}=\mathbf{v}-\mathbf{r}
\end{array}\right.
$$

Moreover, by (4.43) and (4.54) we have:

$$
\sum_{n=0}^{3} A_{n} j^{n}=\rho \Psi-\mathbf{A} \cdot \mathbf{j} \quad \forall\left(x^{0}, x^{1}, x^{2}, x^{3}\right) \in \mathbb{R}^{4} .
$$

Lemma 4.1. Let $\left\{K^{m n}\right\}_{n, m=0,1,2,3}$ be given by (4.29) and $\left\{K_{m n}\right\}_{n, m=0,1,2,3}$ be given by (4.30) Next, let $\left\{F_{m n}\right\}_{n, m=0,1,2,3}$ be an anti-symmetric two-times covariant tensor, defined by

$$
F_{m j}:=\frac{\partial A_{j}}{\partial x^{m}}-\frac{\partial A_{m}}{\partial x^{j}} \quad \forall m, j=0,1,2,3 .
$$

Then, denoting

$$
\begin{aligned}
& \left(x^{0}, x^{1}, x^{2}, x^{3}\right)=\left(x^{0}, \mathbf{x}\right) \quad \text { where } \quad \mathbf{x}:=\left(x^{1}, x^{2}, x^{3}\right) \in \mathbb{R}^{3}, \\
& \left(A_{0}, A_{1}, A_{2}, A_{3}\right)=(\Psi,-\mathbf{A}) \quad \text { where } A_{0}=\Psi \text { and }\left(A_{1}, A_{2}, A_{3}\right)=-\mathbf{A} \text {, } \\
& \left\{\begin{array}{l}
\mathbf{B}:=\left(B_{1}, B_{2}, B_{3}\right):=\operatorname{curl}_{\mathbf{x}} \mathbf{A}, \\
\mathbf{E}:=\left(E_{1}, E_{2}, E_{3}\right):=-\nabla_{\mathbf{x}} \Psi-\frac{\partial \mathbf{A}}{\partial x^{0}}, \\
\mathbf{D}:=\left(D_{1}, D_{2}, D_{3}\right):=\mathbf{E}+\mathbf{v} \times \mathbf{B}, \\
\mathbf{H}:=\left(H_{1}, H_{2}, H_{3}\right):=\mathbf{B}+\mathbf{v} \times \mathbf{D},
\end{array}\right.
\end{aligned}
$$

and

$$
F^{m n}:=\sum_{k=0}^{3} \sum_{j=0}^{3} K^{m j} K^{n k} F_{j k} \quad \forall m, n=0,1,2,3,
$$

we have

$$
\begin{aligned}
& \left\{\begin{array}{l}
F_{00}=0 \\
F_{0 j}=-F_{j 0}=E_{j} \quad \forall j=1,2,3 \\
F_{j j}=0 \quad \forall j=1,2,3 \\
F_{12}=-F_{21}=-B_{3} \\
F_{13}=-F_{31}=B_{2} \\
F_{23}=-F_{32}=-B_{1}, \\
F^{00}=0 \\
F^{0 j}=-F^{j 0}=-D_{j} \quad \forall j=1,2,3 \\
F^{j j}=0 \quad \forall j=1,2,3 \\
F^{12}=-F^{21}=-H_{3} \\
F^{13}=-F^{31}=H_{2} \\
F^{23}=-F^{32}=-H_{1}
\end{array}\right.
\end{aligned}
$$


and

$$
\begin{array}{r}
-\frac{1}{4 \pi}\left(\sum_{n=0}^{3} \sum_{k=0}^{3} \sum_{m=0}^{3} \sum_{p=0}^{3} \frac{1}{4} K^{m n} K^{p k}\left(\frac{\partial A_{p}}{\partial x^{m}}-\frac{\partial A_{m}}{\partial x^{p}}\right)\left(\frac{\partial A_{k}}{\partial x^{n}}-\frac{\partial A_{n}}{\partial x^{k}}\right)\right)=-\frac{1}{4 \pi}\left(\sum_{j=0}^{3} \sum_{k=0}^{3} F^{j k} F_{j k}\right) \\
=\frac{1}{4 \pi}\left(\frac{1}{2}\left|-\nabla_{\mathbf{x}} \Psi-\frac{\partial \mathbf{A}}{\partial x^{0}}+\mathbf{v} \times \operatorname{curl}_{\mathbf{x}} \mathbf{A}\right|^{2}-\frac{1}{2}\left|\operatorname{curl}_{\mathbf{x}} \mathbf{A}\right|^{2}\right) .
\end{array}
$$

Here, for $\mathbf{a}=\left(a_{1}, a_{2}, a_{3}\right) \in \mathbb{R}^{3}$ and $\mathbf{b}=\left(b_{1}, b_{2}, b_{3}\right) \in \mathbb{R}^{3}$ we denote by

$$
\mathbf{a} \times \mathbf{b}:=\left(a_{2} b_{3}-a_{3} b_{2}, a_{3} b_{1}-a_{1} b_{3}, a_{1} b_{2}-b_{2} b_{1}\right) \in \mathbb{R}^{3},
$$

their vector product and, given a vector valued function $\mathbf{f}(\mathbf{x})=\left(f_{1}(\mathbf{x}), f_{2}(\mathbf{x}), f_{3}(\mathbf{x})\right): \mathbb{R}^{3} \rightarrow \mathbb{R}^{3}$ we denote by

$$
\operatorname{curl}_{\mathbf{x}} \mathbf{f}(\mathbf{x}):=\left(\frac{\partial f_{3}}{\partial x^{2}}-\frac{\partial f_{2}}{\partial x^{3}}, \frac{\partial f_{1}}{\partial x^{3}}-\frac{\partial f_{3}}{\partial x^{1}}, \frac{\partial f_{2}}{\partial x^{1}}-\frac{\partial f_{1}}{\partial x^{2}}\right)(\mathbf{x}) .
$$

Similarly to (4.65), by (4.55) we have

$$
\begin{gathered}
\frac{1}{4 \pi G}\left(\sum_{n=0}^{3} \sum_{k=0}^{3} \sum_{m=0}^{3} \sum_{p=0}^{3} \frac{1}{4} K^{m n} K^{p k}\left(\frac{\partial S_{p}}{\partial x^{m}}-\frac{\partial S_{m}}{\partial x^{p}}\right)\left(\frac{\partial S_{k}}{\partial x^{n}}-\frac{\partial S_{n}}{\partial x^{k}}\right)\right) \\
=\frac{1}{4 \pi G}\left(\frac{1}{2}\left|\operatorname{curl}_{\mathbf{x}} \mathbf{S}\right|^{2}-\frac{1}{2}\left|-\nabla_{\mathbf{x}} \Phi-\frac{\partial \mathbf{S}}{\partial x^{0}}+\mathbf{v} \times \operatorname{curl}_{\mathbf{x}} \mathbf{S}\right|^{2}\right)= \\
\frac{1}{4 \pi G}\left(\frac{1}{2}\left|\operatorname{curl}_{\mathbf{x}}(\mathbf{v}-\mathbf{r})\right|^{2}-\frac{1}{2}\left|-\frac{\partial}{\partial x^{0}}(\mathbf{v}-\mathbf{r})-\nabla_{\mathbf{x}}\left(\frac{1}{2}|\mathbf{v}|^{2}-\frac{1}{2}|\mathbf{r}|^{2}\right)+\mathbf{v} \times \operatorname{curl}_{\mathbf{x}}(\mathbf{v}-\mathbf{r})\right|^{2}\right),
\end{gathered}
$$

where we use (4.56) in the last equality. Consequently, by (2.219) and (4.31) we write the simplified total Lagrangian in (2.219) and (2.220) in the given cartesian coordinate system, as

$$
L_{\text {total }}(\mathbf{v},(\Psi,-\mathbf{A}))=\iiint_{\mathcal{V}} \mathcal{L}_{\text {total }}\left(\mathbf{v},(\Psi,-\mathbf{A}),\left(x^{0}, x^{1}, x^{2}, x^{3}\right)\right) d x^{0} d x^{1} d x^{2} d x^{3},
$$

where, using (4.68), (4.65), (4.57) and (4.52) we can rewrite the total Lagrangian density $\mathcal{L}_{\text {total }}\left(\mathbf{v},(\Psi,-\mathbf{A}),\left(x^{0}, x^{1}, x^{2}, x^{3}\right)\right)$ in $(2.220)$ as:

$$
\begin{aligned}
& \mathcal{L}_{\text {total }}\left(\mathbf{v},(\Psi,-\mathbf{A}),\left(x^{0}, x^{1}, x^{2}, x^{3}\right)\right)= \\
& \frac{1}{4 \pi}\left(\frac{1}{2}\left|-\nabla_{\mathbf{x}} \Psi-\frac{\partial \mathbf{A}}{\partial x^{0}}+\mathbf{v} \times \operatorname{curl}_{\mathbf{x}} \mathbf{A}\right|^{2}-\frac{1}{2}\left|\operatorname{curl}_{\mathbf{x}} \mathbf{A}\right|^{2}\right)-\left(\Psi \rho\left(x^{0}, x^{1}, x^{2}, x^{3}\right)-\mathbf{A} \cdot \mathbf{j}\left(x^{0}, x^{1}, x^{2}, x^{3}\right)\right) \\
& \quad+\frac{1}{4 \pi G}\left(\frac{1}{2}\left|\operatorname{curl}_{\mathbf{x}}(\mathbf{v}-\mathbf{r})\right|^{2}-\frac{1}{2}\left|-\frac{\partial}{\partial x^{0}}(\mathbf{v}-\mathbf{r})-\nabla_{\mathbf{x}}\left(\frac{1}{2}|\mathbf{v}|^{2}-\frac{1}{2}|\mathbf{r}|^{2}\right)+\mathbf{v} \times \operatorname{curl}_{\mathbf{x}}(\mathbf{v}-\mathbf{r})\right|^{2}\right) \\
& \quad-\sum_{k=1}^{N} m_{k} \mathcal{G}\left(1-\sum_{j=1}^{3}\left(\frac{d \chi_{k}^{j}}{d x^{0}}\left(x^{0}\right)-v^{j}\left(\chi_{k}\left(x^{0}\right)\right)\right)^{2}\right) \delta\left(x^{1}-\chi_{k}^{1}\left(\chi^{0}\right), x^{2}-\chi_{k}^{2}\left(\chi^{0}\right), x^{3}-\chi_{k}^{3}\left(\chi^{0}\right)\right) .
\end{aligned}
$$


Then, by (4.70) we rewrite (4.69) in the case $\mathcal{V}=\mathbb{R}^{4}$ as:

$$
\begin{gathered}
L_{\text {total }}(\mathbf{v},(\Psi,-\mathbf{A}))=\iiint_{\mathbb{R}^{4}} \frac{1}{4 \pi}\left(\frac{1}{2}\left|-\nabla_{\mathbf{x}} \Psi-\frac{\partial \mathbf{A}}{\partial x^{0}}+\mathbf{v} \times \operatorname{curl}_{\mathbf{x}} \mathbf{A}\right|^{2}-\frac{1}{2}\left|\operatorname{curl}_{\mathbf{x}} \mathbf{A}\right|^{2}\right) d x^{0} \ldots d x^{3} \\
-\iiint \int_{\mathbb{R}^{4}}\left(\Psi \rho\left(x^{0}, x^{1}, x^{2}, x^{3}\right)-\mathbf{A} \cdot \mathbf{j}\left(x^{0}, x^{1}, x^{2}, x^{3}\right)\right) d x^{0} d x^{1} d x^{2} d x^{3}+\iiint \int_{\mathbb{R}^{4}} \frac{1}{4 \pi G}\{ \\
\left.\frac{1}{2}\left|\operatorname{curl}_{\mathbf{x}}(\mathbf{v}-\mathbf{r})\right|^{2}-\frac{1}{2}\left|-\frac{\partial}{\partial x^{0}}(\mathbf{v}-\mathbf{r})-\nabla_{\mathbf{x}}\left(\frac{1}{2}|\mathbf{v}|^{2}-\frac{1}{2}|\mathbf{r}|^{2}\right)+\mathbf{v} \times \operatorname{curl}_{\mathbf{x}}(\mathbf{v}-\mathbf{r})\right|^{2}\right\} d x^{0} d x^{1} d x^{2} d x^{3} \\
+\sum_{k=1}^{N} \int_{-\infty}^{+\infty}\left\{-m_{k} \mathcal{G}\left(1-\sum_{j=1}^{3}\left(\frac{d \chi_{k}^{j}}{d x^{0}}\left(x^{0}\right)-v^{j}\left(\chi_{k}\left(x^{0}\right)\right)\right)^{2}\right)\right\} d x^{0} .
\end{gathered}
$$

In particular, if we assume that our coordinate system is inertial, in addition to being cartesian, then since $\mathbf{r}$, given as in (4.28) and (4.5) by

$$
\begin{aligned}
& \left(r^{0}, r^{1}, r^{2}, r^{3}\right)=\left(1, r^{1}, r^{2}, r^{3}\right)=(1, \mathbf{r}) \quad \text { where } \quad \mathbf{r}:=\left(r^{1}, r^{2}, r^{3}\right) \in \mathbb{R}^{3} \quad \text { with } \\
& r^{m}:=\sum_{j=0}^{3} J^{m j} \frac{\partial \varphi}{\partial x^{j}} \quad \forall m=0,1,2,3,
\end{aligned}
$$

is $\underline{\text { constant }}$ in every inertial coordinate system, which is independent on the point $\left(x^{0}, x^{1}, x^{2}, x^{3}\right) \in$ $\mathbb{R}^{4}$, we simplify the total Lagrangian density $\mathcal{L}_{\text {total }}\left(\mathbf{v},(\Psi,-\mathbf{A}),\left(x^{0}, x^{1}, x^{2}, x^{3}\right)\right)$ in $(4.70)$ as:

$$
\begin{aligned}
& \mathcal{L}_{\text {total }}\left(\mathbf{v},(\Psi,-\mathbf{A}),\left(x^{0}, x^{1}, x^{2}, x^{3}\right)\right)= \\
& \frac{1}{4 \pi}\left(\frac{1}{2}\left|-\nabla_{\mathbf{x}} \Psi-\frac{\partial \mathbf{A}}{\partial x^{0}}+\mathbf{v} \times \operatorname{curl}_{\mathbf{x}} \mathbf{A}\right|^{2}-\frac{1}{2}\left|\operatorname{curl}_{\mathbf{x}} \mathbf{A}\right|^{2}\right)-\left(\Psi \rho\left(x^{0}, x^{1}, x^{2}, x^{3}\right)-\mathbf{A} \cdot \mathbf{j}\left(x^{0}, x^{1}, x^{2}, x^{3}\right)\right) \\
& +\frac{1}{4 \pi G}\left(\frac{1}{2}\left|\operatorname{curl}_{\mathbf{x}} \mathbf{v}\right|^{2}-\frac{1}{2}\left|\frac{\partial \mathbf{v}}{\partial x^{0}}+\nabla_{\mathbf{x}}\left(\frac{1}{2}|\mathbf{v}|^{2}\right)-\mathbf{v} \times \operatorname{curl}_{\mathbf{x}} \mathbf{v}\right|^{2}\right) \\
& -\sum_{k=1}^{N} m_{k} \mathcal{G}\left(1-\sum_{j=1}^{3}\left(\frac{d \chi_{k}^{j}}{d x^{0}}\left(x^{0}\right)-v^{j}\left(\chi_{k}\left(x^{0}\right)\right)\right)^{2}\right) \delta\left(x^{1}-\chi_{k}^{1}\left(\chi^{0}\right), x^{2}-\chi_{k}^{2}\left(\chi^{0}\right), x^{3}-\chi_{k}^{3}\left(\chi^{0}\right)\right) \text {, }
\end{aligned}
$$

and the total Lagrangian in (4.71) as:

$$
\begin{gathered}
L_{\text {total }}(\mathbf{v},(\Psi,-\mathbf{A}))=\iiint \int_{\mathbb{R}^{4}} \frac{1}{4 \pi}\left(\frac{1}{2}\left|-\nabla_{\mathbf{x}} \Psi-\frac{\partial \mathbf{A}}{\partial x^{0}}+\mathbf{v} \times \operatorname{curl}_{\mathbf{x}} \mathbf{A}\right|^{2}-\frac{1}{2}\left|\operatorname{curl}_{\mathbf{x}} \mathbf{A}\right|^{2}\right) d x^{0} d x^{1} d x^{2} d x^{3} \\
-\iiint \int\left(\Psi \rho\left(x^{0}, x^{1}, x^{2}, x^{3}\right)-\mathbf{A} \cdot \mathbf{j}\left(x^{0}, x^{1}, x^{2}, x^{3}\right)\right) d x^{0} d x^{1} d x^{2} d x^{3} \\
+\iiint \int_{\mathbb{R}^{4}} \frac{1}{4 \pi G}\left(\frac{1}{2}\left|\operatorname{curl}_{\mathbf{x}} \mathbf{v}\right|^{2}-\frac{1}{2}\left|\frac{\partial \mathbf{v}}{\partial x^{0}}+\nabla_{\mathbf{x}}\left(\frac{1}{2}|\mathbf{v}|^{2}\right)-\mathbf{v} \times \operatorname{curl}_{\mathbf{x}} \mathbf{v}\right|^{2}\right) d x^{0} d x^{1} d x^{2} d x^{3} \\
+\sum_{k=1}^{N} \int_{-\infty}^{+\infty}\left\{-m_{k} \mathcal{G}\left(1-\sum_{j=1}^{3}\left(\frac{d \chi_{k}^{j}}{d x^{0}}\left(x^{0}\right)-v^{j}\left(\chi_{k}\left(x^{0}\right)\right)\right)^{2}\right)\right\} d x^{0} .
\end{gathered}
$$


So, in the coordinate system which is simultaneously inertial and cartesian (we can obtain such systems by the Galilean Transformations from the kinematically preferable coordinate system) neither the total Lagrangian density in (4.73) nor the total Lagrangian in (4.74) are dependent on the kinematical pseudometric $\left\{J^{m n}\right\}_{0 \leq m, n \leq 3}$ and/or on the contravariant four-vector of inertia $\left(r^{0}, r^{1}, r^{2}, r^{3}\right)$.

\section{The Euler-Lagrange for the Lagrangian of the motion of a classical point particle in a cartesian coordinate system}

Consider a cartesian coordinate system and a classical point particle with the inertial mass $m$ and the charge $\sigma$ moving in the generalized-gravitational field given by a contravariant pseudo-metric $\left\{K^{m n}\right\}_{n, m=0,1,2,3}$, satisfying (4.29), so that

$$
\left\{\begin{array}{l}
K^{00}=1 \\
K^{j m}=-\delta_{j m}+v^{j} v^{m} \quad \forall 1 \leq j, m \leq 3 \\
K^{0 j}=K^{j 0}=v^{j} \quad \forall 1 \leq j \leq 3,
\end{array}\right.
$$

and the electromagnetic field with the four-covector of the electromagnetic potential $\left(A_{0}, A_{1}, A_{2}, A_{3}\right)$. Next assume that $\chi\left(x^{0}\right):=\left(x^{0}, \chi^{1}\left(x^{0}\right), \chi^{2}\left(x^{0}\right), \chi^{3}\left(x^{0}\right)\right):[a, b] \rightarrow \mathbb{R}^{4} \in \mathbb{R}^{4}$ is a four-dimensional space-time trajectory of the particle, parameterized by the first coordinate $x^{0}$ in the interval $x^{0} \in$ $[a, b]$ (including the cases where $a=-\infty$ and/or $b=+\infty$ ). Then, since $\varphi=x^{0}+$ Const, we rewrite the Lagrangian of motion of this particle in (2.100) as:

$$
L_{\mathcal{G}}(\chi)=\int_{a}^{b}\left\{-m \mathcal{G}\left(\sum_{j=0}^{3} \sum_{k=0}^{3} K_{j k}\left(\chi\left(x^{0}\right)\right) \frac{d \chi^{j}}{d x^{0}} \frac{d \chi^{k}}{d x^{0}}\right)-\sum_{j=0}^{3} \sigma A_{j}\left(\chi\left(x^{0}\right)\right) \frac{d \chi^{j}}{d x^{0}}\right\} d x^{0},
$$

where $\left\{K_{m n}\right\}_{n, m=0,1,2,3}$ is given as in (4.30) by

$$
\left\{\begin{array}{l}
K_{00}=1-|\mathbf{v}|^{2} \\
K_{j m}=-\delta_{j m} \quad \forall 1 \leq j, m \leq 3 \\
K_{0 j}=K_{j 0}=v^{j} \quad \forall 1 \leq j \leq 3,
\end{array}\right.
$$

with

$$
\left(v^{0}, v^{1}, v^{2}, v^{3}\right)=\left(1, v^{1}, v^{2}, v^{3}\right)=(1, \mathbf{v}) \quad \text { where } \quad \mathbf{v}:=\left(v^{1}, v^{2}, v^{3}\right) \in \mathbb{R}^{3} .
$$

Then, since $\chi^{0}\left(x^{0}\right)=x^{0}$, by (5.3) we rewrite (5.2) as:

$$
\begin{aligned}
L_{\mathcal{G}}(\mathbf{z})=\int_{a}^{b}\left\{-m \mathcal{G}\left(1-\left|\frac{d \mathbf{z}}{d x^{0}}\left(x^{0}\right)-\mathbf{v}\left(x^{0}, \mathbf{z}\left(x^{0}\right)\right)\right|^{2}\right)\right\} d x^{0} \\
-\int_{a}^{b}\left\{\sigma\left(\Psi\left(x^{0}, \mathbf{z}\left(x^{0}\right)\right)-\mathbf{A}\left(x^{0}, \mathbf{z}\left(x^{0}\right)\right) \cdot \frac{d \mathbf{z}}{d x^{0}}\left(x^{0}\right)\right)\right\} d x^{0} .
\end{aligned}
$$


where we denote

$$
\mathbf{z}\left(x^{0}\right):=\left(\chi^{1}\left(x^{0}\right), \chi^{2}\left(x^{0}\right), \chi^{3}\left(x^{0}\right)\right) \in \mathbb{R}^{3} \quad \forall x^{0} \in[a, b],
$$

and

$$
\left(A_{0}, A_{1}, A_{2}, A_{3}\right):=(\Psi,-\mathbf{A}) \quad \text { where } \quad A_{0}=\Psi \text { and }\left(A_{1}, A_{2}, A_{3}\right)=-\mathbf{A} .
$$

Next, we investigate the critical points $\mathbf{z}\left(x^{0}\right)$ of (5.5). Then, by the Euler Lagrange we have:

$$
\frac{\delta L_{\mathcal{G}}}{\delta \mathbf{z}}(\mathbf{z})=0 \text {. }
$$

Therefore,

$$
\begin{aligned}
0= & -m \frac{d}{d x^{0}}\left\{2 \mathcal{G}^{\prime}\left(1-\left|\frac{d \mathbf{z}}{d x^{0}}\left(x^{0}\right)-\mathbf{v}\left(x^{0}, \mathbf{z}\left(x^{0}\right)\right)\right|^{2}\right)\left(\frac{d \mathbf{z}}{d x^{0}}\left(x^{0}\right)-\mathbf{v}\left(x^{0}, \mathbf{z}\left(x^{0}\right)\right)\right)\right\} \\
-2 m \mathcal{G}^{\prime} & \left(1-\left|\frac{d \mathbf{z}}{d x^{0}}\left(x^{0}\right)-\mathbf{v}\left(x^{0}, \mathbf{z}\left(x^{0}\right)\right)\right|^{2}\right)\left\{\nabla_{\mathbf{x}} \mathbf{v}\left(x^{0}, \mathbf{z}\left(x^{0}\right)\right)\right\}^{T} \cdot\left(\frac{d \mathbf{z}}{d x^{0}}\left(x^{0}\right)-\mathbf{v}\left(x^{0}, \mathbf{z}\left(x^{0}\right)\right)\right) \\
& -\sigma\left(\frac{d}{d x^{0}}\left\{\mathbf{A}\left(x^{0}, \mathbf{z}\left(x^{0}\right)\right)\right\}+\nabla_{\mathbf{x}} \Psi\left(x^{0}, \mathbf{z}\left(x^{0}\right)\right)-\left\{d_{\mathbf{x}} \mathbf{A}\left(x^{0}, \mathbf{z}\left(x^{0}\right)\right)\right\}^{T} \cdot \frac{d \mathbf{z}}{d x^{0}}\left(x^{0}\right)\right),
\end{aligned}
$$

and, by the following well known identity from the Vector Analysis:

$$
\mathbf{f} \times\left(\operatorname{curl}_{\mathbf{x}} \mathbf{g}\right)=\left(d_{\mathbf{x}} \mathbf{g}\right)^{T} \cdot \mathbf{f}-\left(d_{\mathbf{x}} \mathbf{g}\right) \cdot \mathbf{f} \quad \forall \mathbf{f}(\mathbf{x}), \mathbf{g}(\mathbf{x}): \mathbb{R}^{3} \rightarrow \mathbb{R}^{3},
$$

we rewrite (5.9) as:

$$
\begin{gathered}
0=-m \frac{d}{d x^{0}}\left\{2 \mathcal{G}^{\prime}\left(1-\left|\frac{d \mathbf{z}}{d x^{0}}\left(x^{0}\right)-\mathbf{v}\left(x^{0}, \mathbf{z}\left(x^{0}\right)\right)\right|^{2}\right)\right\}\left(\frac{d \mathbf{z}}{d x^{0}}\left(x^{0}\right)-\mathbf{v}\left(x^{0}, \mathbf{z}\left(x^{0}\right)\right)\right) \\
-2 m \mathcal{G}^{\prime}\left(1-\left|\frac{d \mathbf{z}}{d x^{0}}\left(x^{0}\right)-\mathbf{v}\left(x^{0}, \mathbf{z}\left(x^{0}\right)\right)\right|^{2}\right) \frac{d^{2} \mathbf{z}}{d\left(x^{0}\right)^{2}}\left(x^{0}\right) \\
+2 m \mathcal{G}^{\prime}\left(1-\left|\frac{d \mathbf{z}}{d x^{0}}\left(x^{0}\right)-\mathbf{v}\left(x^{0}, \mathbf{z}\left(x^{0}\right)\right)\right|^{2}\right)\left(\left\{\frac{\partial}{\partial x^{0}} \mathbf{v}+\nabla_{\mathbf{x}}\left(\frac{1}{2}|\mathbf{v}|^{2}\right)-\frac{d \mathbf{z}}{d x^{0}} \times \operatorname{curl}_{\mathbf{x}} \mathbf{v}\right\}\left(x^{0}, \mathbf{z}\left(x^{0}\right)\right)\right) \\
+\sigma\left(\left\{-\frac{\partial \mathbf{A}}{\partial x^{0}}-\nabla_{\mathbf{x}} \Psi+\frac{d \mathbf{z}}{d x^{0}}\left(x^{0}\right) \times \operatorname{curl}_{\mathbf{x}} \mathbf{A}\right\}\left(x^{0}, \mathbf{z}\left(x^{0}\right)\right)\right) .
\end{gathered}
$$

So, denoting

$$
\left\{\begin{array}{l}
\mathbf{E}=-\nabla_{\mathbf{x}} \Psi-\frac{\partial \mathbf{A}}{\partial x^{0}} \\
\mathbf{B}=\operatorname{curl}_{\mathbf{x}} \mathbf{A}
\end{array}\right.
$$

we rewrite (5.11) as

$$
\begin{aligned}
& 2 m \mathcal{G}^{\prime}\left(1-\left|\frac{d \mathbf{z}}{d x^{0}}\left(x^{0}\right)-\mathbf{v}\left(x^{0}, \mathbf{z}\left(x^{0}\right)\right)\right|^{2}\right) \frac{d^{2} \mathbf{z}}{d\left(x^{0}\right)^{2}}\left(x^{0}\right)= \\
&-m \frac{d}{d x^{0}}\left\{2 \mathcal{G}^{\prime}\left(1-\left|\frac{d \mathbf{z}}{d x^{0}}\left(x^{0}\right)-\mathbf{v}\left(x^{0}, \mathbf{z}\left(x^{0}\right)\right)\right|^{2}\right)\right\}\left(\frac{d \mathbf{z}}{d x^{0}}\left(x^{0}\right)-\mathbf{v}\left(x^{0}, \mathbf{z}\left(x^{0}\right)\right)\right) \\
&+2 m \mathcal{G}^{\prime}\left(1-\left|\frac{d \mathbf{z}}{d x^{0}}\left(x^{0}\right)-\mathbf{v}\left(x^{0}, \mathbf{z}\left(x^{0}\right)\right)\right|^{2}\right)\left(\left\{\frac{\partial}{\partial x^{0}} \mathbf{v}+\nabla_{\mathbf{x}}\left(\frac{1}{2}|\mathbf{v}|^{2}\right)-\frac{d \mathbf{z}}{d x^{0}} \times \operatorname{curl}_{\mathbf{x}} \mathbf{v}\right\}\left(x^{0}, \mathbf{z}\left(x^{0}\right)\right)\right) \\
&+\sigma\left(\mathbf{E}\left(x^{0}, \mathbf{z}\left(x^{0}\right)\right)+\frac{d \mathbf{z}}{d x^{0}}\left(x^{0}\right) \times \mathbf{B}\left(x^{0}, \mathbf{z}\left(x^{0}\right)\right)\right) .
\end{aligned}
$$

However, as before, here we consider two cases: 
- The case of non-relativistic approximation, where $\mathcal{G}(\tau):=\frac{1}{2}(\tau-1)$.

- The case of relativistic particles, where $\mathcal{G}(\tau):=\sqrt{\tau}-1$.

In the first case we have $2 \mathcal{G}^{\prime}(\tau)=1$. In the second case we have $2 \mathcal{G}^{\prime}(\tau)=\tau^{-\frac{1}{2}}=1+\mathcal{O}(\tau-1)$ and so $2 \mathcal{G}^{\prime}(\tau) \approx 1$ in the non-relativistic approximation where

$$
\left|\frac{d \mathbf{z}}{d x^{0}}-\mathbf{v}\right|^{2} \ll 1
$$

Thus, in the latter case we rewrite (5.13) as

$$
\begin{aligned}
m \frac{d^{2} \mathbf{z}}{d\left(x^{0}\right)^{2}}\left(x^{0}\right)=m\left(\frac{\partial}{\partial x^{0}} \mathbf{v}\left(x^{0}, \mathbf{z}\left(x^{0}\right)\right)+\right. & \left.\nabla_{\mathbf{x}}\left(\frac{1}{2}\left|\mathbf{v}\left(x^{0}, \mathbf{z}\left(x^{0}\right)\right)\right|^{2}\right)-\frac{d \mathbf{z}}{d x^{0}}\left(x^{0}\right) \times \operatorname{curl}_{\mathbf{x}} \mathbf{v}\left(x^{0}, \mathbf{z}\left(x^{0}\right)\right)\right) \\
& +\sigma\left(\mathbf{E}\left(x^{0}, \mathbf{z}\left(x^{0}\right)\right)+\frac{d \mathbf{z}}{d x^{0}}\left(x^{0}\right) \times \mathbf{B}\left(x^{0}, \mathbf{z}\left(x^{0}\right)\right)\right) .
\end{aligned}
$$

\section{The Euler-Lagrange for the Lagrangian of the gravitational and Electromagnetic fields in (4.71) in a cartesian coordi- nate system}

Lemma 6.1. Consider the strongly correlated kinematical contravariant pseudo-metric

$\left\{J^{m n}\right\}_{m, n=0,1,2,3}$ and the kinematical global time $\varphi$, forming a standard kinematical Lorentz's structure with global time on $\mathbb{R}^{4}$ as in Proposition 2.7 and Remark 2.6. Next, let $\left(r^{0}, r^{1}, r^{2}, r^{3}\right)$ be the contravariant four-vector of the inertia, corresponding to $\left\{J^{m n}\right\}_{m, n=0,1,2,3}$ and $\varphi$, defined by

$$
r^{m}:=\sum_{j=0}^{3} J^{m j} \frac{\partial \varphi}{\partial x^{j}} \quad \forall m=0,1,2,3 .
$$

and denote

$$
\left(r^{0}, r^{1}, r^{2}, r^{3}\right):=\left(r^{0}, \mathbf{r}\right) \quad \text { where } \quad \mathbf{r}:=\left(r^{1}, r^{2}, r^{3}\right) \in \mathbb{R}^{3} .
$$

Then, in a cartesian coordinate system we have:

$$
\left\{\begin{array}{l}
r^{0}=1 \\
\left(d_{\mathbf{x}} \mathbf{r}+\left\{d_{\mathbf{x}} \mathbf{r}\right\}^{T}\right):=\left(\left\{\frac{\partial r^{m}}{\partial x^{j}}+\frac{\partial r^{j}}{\partial x^{m}}\right\}_{m, j=1,2,3}\right)=0 \\
\frac{\partial^{2} r^{m}}{\partial x^{j} \partial x^{n}}=0 \quad \forall j, m, n=1,2,3 \\
\operatorname{div}_{\mathbf{x}} \mathbf{r}=0,
\end{array}\right.
$$

where we denote

$$
\left(x^{0}, x^{1}, x^{2}, x^{3}\right):=\left(x^{0}, \mathbf{x}\right) \quad \text { with } \quad \mathbf{x}:=\left(x^{1}, x^{2}, x^{3}\right) \in \mathbb{R}^{3} .
$$


We investigate critical points of the functional in (4.71). As before, we denote

$$
\left(A_{0}, A_{1}, A_{2}, A_{3}\right)=(\Psi,-\mathbf{A}) \quad \text { where } A_{0}=\Psi \text { and }\left(A_{1}, A_{2}, A_{3}\right)=-\mathbf{A},
$$

and

$$
\left(S_{0}, S_{1}, S_{2}, S_{3}\right):=-(\Phi,-\mathbf{h}) \quad \text { where } \quad S_{0}=-\Phi \quad \text { and } \quad\left(S_{1}, S_{2}, S_{3}\right)=\mathbf{h} \text {. }
$$

In particular, by (4.36) we have:

$$
\left\{\begin{array}{l}
\Phi=\frac{1}{2}\left(|\mathbf{v}|^{2}-|\mathbf{r}|^{2}\right) \\
\mathbf{h}=\mathbf{v}-\mathbf{r}
\end{array}\right.
$$

Furthermore, we denote

$$
\left\{\begin{array}{l}
\mathbf{B}=\operatorname{curl}_{\mathbf{x}} \mathbf{A} \\
\mathbf{D}=-\nabla_{\mathbf{x}} \Psi-\frac{\partial \mathbf{A}}{\partial x^{0}}+\mathbf{v} \times \operatorname{curl}_{\mathbf{x}} \mathbf{A} \\
\mathbf{E}=-\nabla_{\mathbf{x}} \Psi-\frac{\partial \mathbf{A}}{\partial x^{0}}=\mathbf{D}-\mathbf{v} \times \mathbf{B} \\
\mathbf{H}=\operatorname{curl}_{\mathbf{x}} \mathbf{A}+\mathbf{v} \times\left(-\nabla_{\mathbf{x}} \Psi-\frac{\partial \mathbf{A}}{\partial x^{0}}+\mathbf{v} \times \operatorname{curl}_{\mathbf{x}} \mathbf{A}\right)=\mathbf{B}+\mathbf{v} \times \mathbf{D}
\end{array}\right.
$$

Moreover, we define

$$
\left\{\begin{array}{l}
\mathbf{R}=-\nabla_{\mathbf{x}} \Phi-\frac{\partial \mathbf{h}}{\partial x^{0}}+\mathbf{v} \times \operatorname{curl}_{\mathbf{x}} \mathbf{h} \\
\mathbf{Q}=\operatorname{curl}_{\mathbf{x}} \mathbf{h}
\end{array}\right.
$$

and by $(6.7)$ we rewrite $(6.9)$ as:

$$
\left\{\begin{array}{l}
\mathbf{R}=-\frac{\partial}{\partial x^{0}}(\mathbf{v}-\mathbf{r})-\nabla_{\mathbf{x}}\left(\frac{1}{2}|\mathbf{v}|^{2}-\frac{1}{2}|\mathbf{r}|^{2}\right)+\mathbf{v} \times \operatorname{curl}_{\mathbf{x}}(\mathbf{v}-\mathbf{r}) \\
\mathbf{Q}=\operatorname{curl}_{\mathbf{x}}(\mathbf{v}-\mathbf{r}) .
\end{array}\right.
$$

We also can rewrite (6.10) as:

$$
\begin{aligned}
& \mathbf{Q}=\operatorname{curl}_{\mathbf{x}}(\mathbf{v}-\mathbf{r}) \quad \text { and } \quad \mathbf{R}= \\
& \left(\frac{\partial \mathbf{r}}{\partial x^{0}}+\nabla_{\mathbf{x}}\left(\frac{1}{2}|\mathbf{r}|^{2}\right)-\mathbf{r} \times \operatorname{curl}_{\mathbf{x}} \mathbf{r}\right)-\left(\frac{\partial \mathbf{v}}{\partial x^{0}}+\nabla_{\mathbf{x}}\left(\frac{1}{2}|\mathbf{v}|^{2}\right)-\mathbf{v} \times \operatorname{curl}_{\mathbf{x}} \mathbf{v}\right)-(\mathbf{v}-\mathbf{r}) \times \operatorname{curl}_{\mathbf{x}} \mathbf{r} \\
& =\left(\frac{\partial \mathbf{r}}{\partial x^{0}}+d_{\mathbf{x}} \mathbf{r} \cdot \mathbf{r}\right)-\left(\frac{\partial \mathbf{v}}{\partial x^{0}}+d_{\mathbf{x}} \mathbf{v} \cdot \mathbf{v}\right)-(\mathbf{v}-\mathbf{r}) \times \operatorname{curl}_{\mathbf{x}} \mathbf{r}
\end{aligned}
$$

where in the last equality we use the following well known identity from the Vector Analysis:

$$
\mathbf{f} \times\left(\operatorname{curl}_{\mathbf{x}} \mathbf{g}\right)=\left(d_{\mathbf{x}} \mathbf{g}\right)^{T} \cdot \mathbf{f}-\left(d_{\mathbf{x}} \mathbf{g}\right) \cdot \mathbf{f} \quad \forall \mathbf{f}(\mathbf{x}), \mathbf{g}(\mathbf{x}): \mathbb{R}^{3} \rightarrow \mathbb{R}^{3}
$$

Moreover, in inertial coordinate system where $d_{\mathbf{x}} \mathbf{r}=0$ and $\frac{\partial \mathbf{r}}{\partial x^{0}}=0$ we simplify $(6.11)$ as:

$$
\left\{\begin{array}{l}
\mathbf{R}=-\frac{\partial \mathbf{v}}{\partial x^{0}}-\nabla_{\mathbf{x}}\left(\frac{1}{2}|\mathbf{v}|^{2}\right)+\mathbf{v} \times \operatorname{curl}_{\mathbf{x}} \mathbf{v}=-\left(\frac{\partial \mathbf{v}}{\partial x^{0}}+d_{\mathbf{x}} \mathbf{v} \cdot \mathbf{v}\right) \\
\mathbf{Q}=\operatorname{curl}_{\mathbf{x}} \mathbf{v} .
\end{array}\right.
$$


Furthermore, by (6.8) and (6.9) we have:

$$
\left\{\begin{array}{l}
\operatorname{curl}_{\mathbf{x}} \mathbf{E}+\frac{\partial \mathbf{B}}{\partial x^{0}}=\operatorname{curl}_{\mathbf{x}} \mathbf{D}+\frac{\partial \mathbf{B}}{\partial x^{0}}-\operatorname{curl}_{\mathbf{x}}(\mathbf{v} \times \mathbf{B})=0 \\
\operatorname{div}_{\mathbf{x}} \mathbf{B}=0 \\
\operatorname{curl}_{\mathbf{x}} \mathbf{R}+\frac{\partial \mathbf{Q}}{\partial x^{0}}-\operatorname{curl}_{\mathbf{x}}(\mathbf{v} \times \mathbf{Q})=0 \\
\operatorname{div}_{\mathbf{x}} \mathbf{Q}=0 .
\end{array}\right.
$$

Next, taking the divergence by $\mathbf{x}$ of $(6.11)$ we deduce

$$
\begin{aligned}
& \operatorname{div}_{\mathbf{x}} \mathbf{R}=\left(\frac{\partial}{\partial x^{0}}\left\{\operatorname{div}_{\mathbf{x}} \mathbf{r}\right\}+\mathbf{r} \cdot \nabla_{\mathbf{x}}\left\{\operatorname{div}_{\mathbf{x}} \mathbf{r}\right\}+\sum_{j=1}^{3} \sum_{m=1}^{3} \frac{\partial r^{m}}{\partial x^{j}} \frac{\partial r^{j}}{\partial x^{m}}\right) \\
& -\left(\frac{\partial}{\partial x^{0}}\left\{\operatorname{div}_{\mathbf{x}} \mathbf{v}\right\}+\mathbf{v} \cdot \nabla_{\mathbf{x}}\left\{\operatorname{div}_{\mathbf{x}} \mathbf{v}\right\}+\sum_{j=1}^{3} \sum_{m=1}^{3} \frac{\partial v^{m}}{\partial x^{j}} \frac{\partial v^{j}}{\partial x^{m}}\right)-\operatorname{div}_{\mathbf{x}}\left\{(\mathbf{v}-\mathbf{r}) \times \operatorname{curl}_{\mathbf{x}} \mathbf{r}\right\} \\
& =\left(\frac{\partial}{\partial x^{0}}\left\{\operatorname{div}_{\mathbf{x}} \mathbf{r}\right\}+\mathbf{r} \cdot \nabla_{\mathbf{x}}\left\{\operatorname{div}_{\mathbf{x}} \mathbf{r}\right\}+\sum_{j=1}^{3} \sum_{m=1}^{3} \frac{1}{4}\left(\frac{\partial r^{m}}{\partial x^{j}}+\frac{\partial r^{j}}{\partial x^{m}}\right)^{2}-\sum_{j=1}^{3} \sum_{m=1}^{3} \frac{1}{4}\left(\frac{\partial r^{m}}{\partial x^{j}}-\frac{\partial r^{j}}{\partial x^{m}}\right)^{2}\right) \\
& -\left(\frac{\partial}{\partial x^{0}}\left\{\operatorname{div}_{\mathbf{x}} \mathbf{v}\right\}+\mathbf{v} \cdot \nabla_{\mathbf{x}}\left\{\operatorname{div}_{\mathbf{x}} \mathbf{v}\right\}+\sum_{j=1}^{3} \sum_{m=1}^{3} \frac{1}{4}\left(\frac{\partial v^{m}}{\partial x^{j}}+\frac{\partial v^{j}}{\partial x^{m}}\right)^{2}-\sum_{j=1}^{3} \sum_{m=1}^{3} \frac{1}{4}\left(\frac{\partial v^{m}}{\partial x^{j}}-\frac{\partial v^{j}}{\partial x^{m}}\right)^{2}\right) \\
& -\left(\operatorname{curl}_{\mathbf{x}}\{(\mathbf{v}-\mathbf{r})\}\right) \cdot\left(\operatorname{curl}_{\mathbf{x}} \mathbf{r}\right)+(\mathbf{v}-\mathbf{r}) \cdot\left(\operatorname{curl}_{\mathbf{x}}\left\{c u r l_{\mathbf{x}} \mathbf{r}\right\}\right), \quad(6.15)
\end{aligned}
$$

where in the last equality we use the following well known identity from the Vector Analysis:

$$
\operatorname{div}_{\mathbf{x}}(\mathbf{f} \times \mathbf{g})=\mathbf{g} \cdot \operatorname{curl}_{\mathbf{x}} \mathbf{f}-\mathbf{f} \cdot \operatorname{curl}_{\mathbf{x}} \mathbf{g} \quad \forall \mathbf{f}(\mathbf{x}), \mathbf{g}(\mathbf{x}): \mathbb{R}^{3} \rightarrow \mathbb{R}^{3}
$$

However, we can rewrite (6.15) as:

$$
\begin{aligned}
\operatorname{div}_{\mathbf{x}} \mathbf{R}= & \left(\frac{\partial}{\partial x^{0}}\left\{\operatorname{div}_{\mathbf{x}} \mathbf{r}\right\}+\mathbf{r} \cdot \nabla_{\mathbf{x}}\left\{\operatorname{div}_{\mathbf{x}} \mathbf{r}\right\}+\sum_{j=1}^{3} \sum_{m=1}^{3} \frac{1}{4}\left(\frac{\partial r^{m}}{\partial x^{j}}+\frac{\partial r^{j}}{\partial x^{m}}\right)^{2}\right) \\
& -\left(\frac{\partial}{\partial x^{0}}\left\{\operatorname{div}_{\mathbf{x}} \mathbf{v}\right\}+\mathbf{v} \cdot \nabla_{\mathbf{x}}\left\{\operatorname{div}_{\mathbf{x}} \mathbf{v}\right\}+\sum_{j=1}^{3} \sum_{m=1}^{3} \frac{1}{4}\left(\frac{\partial v^{m}}{\partial x^{j}}+\frac{\partial v^{j}}{\partial x^{m}}\right)^{2}\right) \\
- & \frac{1}{2}\left|c u r l_{\mathbf{x}} \mathbf{r}\right|^{2}+\frac{1}{2}\left|\operatorname{curl}_{\mathbf{x}} \mathbf{v}\right|^{2}-\left(\operatorname{curl}_{\mathbf{x}}\{(\mathbf{v}-\mathbf{r})\}\right) \cdot\left(\operatorname{curl}_{\mathbf{x}} \mathbf{r}\right)+(\mathbf{v}-\mathbf{r}) \cdot\left(\operatorname{curl}_{\mathbf{x}}\left\{\operatorname{curl}_{\mathbf{x}} \mathbf{r}\right\}\right) \\
= & \left(\frac{\partial}{\partial x^{0}}\left\{\operatorname{div}_{\mathbf{x}} \mathbf{r}\right\}+\operatorname{div}_{\mathbf{x}}\left\{\left(\operatorname{div}_{\mathbf{x}} \mathbf{r}\right) \cdot \mathbf{r}\right\}+\sum_{j=1}^{3} \sum_{m=1}^{3} \frac{1}{4}\left(\frac{\partial r^{m}}{\partial x^{j}}+\frac{\partial r^{j}}{\partial x^{m}}\right)^{2}-\left(\operatorname{div}_{\mathbf{x}} \mathbf{r}\right)^{2}\right) \\
- & \left(\frac{\partial}{\partial x^{0}}\left\{\operatorname{div}_{\mathbf{x}} \mathbf{v}\right\}+\operatorname{div}_{\mathbf{x}}\left\{\left(\operatorname{div}_{\mathbf{x}} \mathbf{v}\right) \cdot \mathbf{v}\right\}+\sum_{j=1}^{3} \sum_{m=1}^{3} \frac{1}{4}\left(\frac{\partial v^{m}}{\partial x^{j}}+\frac{\partial v^{j}}{\partial x^{m}}\right)^{2}-\left(\operatorname{div}_{\mathbf{x}} \mathbf{v}\right)^{2}\right) \\
& +\frac{1}{2}\left|c u r l_{\mathbf{x}}(\mathbf{v}-\mathbf{r})\right|^{2}+(\mathbf{v}-\mathbf{r}) \cdot\left(\operatorname{curl}_{\mathbf{x}}\left\{\operatorname{curl}_{\mathbf{x}} \mathbf{r}\right\}\right) .
\end{aligned}
$$


Then, since by (6.11) we have $\operatorname{curl}_{\mathbf{x}}(\mathbf{v}-\mathbf{r})=\mathbf{Q}$, we rewrite (6.17) as:

$$
\begin{aligned}
\left(\frac{\partial}{\partial x^{0}}\left\{\operatorname{div}_{\mathbf{x}} \mathbf{v}\right\}+\operatorname{div}_{\mathbf{x}}\left\{\left(\operatorname{div}_{\mathbf{x}} \mathbf{v}\right) \cdot \mathbf{v}\right\}+\sum_{j=1}^{3}\right. & \left.\sum_{m=1}^{3} \frac{1}{4}\left(\frac{\partial v^{m}}{\partial x^{j}}+\frac{\partial v^{j}}{\partial x^{m}}\right)^{2}-\left(\operatorname{div}_{\mathbf{x}} \mathbf{v}\right)^{2}\right)= \\
\left(\frac{\partial}{\partial x^{0}}\left\{\operatorname{div}_{\mathbf{x}} \mathbf{r}\right\}+\operatorname{div}_{\mathbf{x}}\left\{\left(\operatorname{div}_{\mathbf{x}} \mathbf{r}\right) \cdot \mathbf{r}\right\}\right. & \left.+\sum_{j=1}^{3} \sum_{m=1}^{3} \frac{1}{4}\left(\frac{\partial r^{m}}{\partial x^{j}}+\frac{\partial r^{j}}{\partial x^{m}}\right)^{2}-\left(\operatorname{div}_{\mathbf{x}} \mathbf{r}\right)^{2}\right) \\
& +(\mathbf{v}-\mathbf{r}) \cdot\left(\operatorname{curl}_{\mathbf{x}}\left\{\operatorname{curl}_{\mathbf{x}} \mathbf{r}\right\}\right)+\frac{1}{2}|\mathbf{Q}|^{2}-\operatorname{div}_{\mathbf{x}} \mathbf{R} .
\end{aligned}
$$

Next, by Lemma 6.1 we have

$$
\left\{\begin{array}{l}
\left(d_{\mathbf{x}} \mathbf{r}+\left\{d_{\mathbf{x}} \mathbf{r}\right\}^{T}\right):=\left(\left\{\frac{\partial r^{m}}{\partial x^{j}}+\frac{\partial r^{j}}{\partial x^{m}}\right\}_{m, j=1,2,3}\right)=0 \\
\operatorname{curl}_{\mathbf{x}}\left(\operatorname{curl}_{\mathbf{x}} \mathbf{r}\right)=0 \\
\operatorname{div}_{\mathbf{x}} \mathbf{r}=0
\end{array}\right.
$$

Then, inserting (6.19) into (6.18) and using the identity $\mathbf{Q}=\operatorname{curl}_{\mathbf{x}}(\mathbf{v}-\mathbf{r})$ in (6.11) with (6.19) we obtain:

$$
\begin{aligned}
&\left(\frac{\partial}{\partial x^{0}}\left\{\operatorname{div}_{\mathbf{x}} \mathbf{v}\right\}+\operatorname{div}_{\mathbf{x}}\right.\left.\left\{\left(\operatorname{div}_{\mathbf{x}} \mathbf{v}\right) \cdot \mathbf{v}\right\}+\sum_{j=1}^{3} \sum_{m=1}^{3} \frac{1}{4}\left(\frac{\partial v^{m}}{\partial x^{j}}+\frac{\partial v^{j}}{\partial x^{m}}\right)^{2}-\left(\operatorname{div}_{\mathbf{x}} \mathbf{v}\right)^{2}\right) \\
&=\frac{1}{2}|\mathbf{Q}|^{2}-\operatorname{div}_{\mathbf{x}} \mathbf{R} \quad \text { and } \quad \operatorname{curl}_{\mathbf{x}}\left(\operatorname{curl}_{\mathbf{x}} \mathbf{v}\right)=\operatorname{curl}_{\mathbf{x}} \mathbf{Q} .
\end{aligned}
$$

Next we investigate the Euler-Lagrange of (4.71). We denote

$$
\begin{aligned}
& L_{1}(\mathbf{v},(\Phi,-\mathbf{h}),(\Psi,-\mathbf{A}))= \\
& \iiint \int_{\mathbb{R}^{4}} \frac{1}{4 \pi}\left(\frac{1}{2}\left|-\nabla_{\mathbf{x}} \Psi-\frac{\partial \mathbf{A}}{\partial x^{0}}+\mathbf{v} \times \operatorname{curl}_{\mathbf{x}} \mathbf{A}\right|^{2}-\frac{1}{2}\left|\operatorname{curl}_{\mathbf{x}} \mathbf{A}\right|^{2}\right) d x^{0} d x^{1} d x^{2} d x^{3} \\
& \quad-\iiint \int\left(\Psi \rho\left(x^{0}, x^{1}, x^{2}, x^{3}\right)-\mathbf{A} \cdot \mathbf{j}\left(x^{0}, x^{1}, x^{2}, x^{3}\right)\right) d x^{0} d x^{1} d x^{2} d x^{3} \\
& +\iiint \int_{\mathbb{R}^{4}} \frac{1}{4 \pi G}\left(\frac{1}{2}\left|\operatorname{curl}_{\mathbf{x}} \mathbf{h}\right|^{2}-\frac{1}{2}\left|-\frac{\partial \mathbf{h}}{\partial x^{0}}-\nabla_{\mathbf{x}} \Phi+\mathbf{v} \times \operatorname{curl}_{\mathbf{x}} \mathbf{h}\right|^{2}\right) d x^{0} d x^{1} d x^{2} d x^{3} \\
& \quad+\sum_{k=1}^{N} \int_{-\infty}^{+\infty}\left\{-m_{k} \mathcal{G}\left(1-\sum_{j=1}^{3}\left(\frac{d \chi_{k}^{j}}{d x^{0}}\left(x^{0}\right)-v^{j}\left(\chi_{k}\left(x^{0}\right)\right)\right)^{2}\right)\right\} d x^{0},
\end{aligned}
$$

where

$$
\left\{\begin{array}{l}
\Phi=\frac{1}{2}\left(|\mathbf{v}|^{2}-|\mathbf{r}|^{2}\right) \\
\mathbf{h}=(\mathbf{v}-\mathbf{r})
\end{array}\right.
$$


so that we have:

$$
\begin{gathered}
L_{1}\left(\mathbf{v},\left(\frac{1}{2}\left(|\mathbf{v}|^{2}-|\mathbf{r}|^{2}\right),-(\mathbf{v}-\mathbf{r})\right),(\Psi,-\mathbf{A})\right)=L_{\text {total }}(\mathbf{v},(\Psi,-\mathbf{A}))= \\
\iiint \int_{\mathbb{R}^{4}} \frac{1}{4 \pi}\left(\frac{1}{2}\left|-\nabla_{\mathbf{x}} \Psi-\frac{\partial \mathbf{A}}{\partial x^{0}}+\mathbf{v} \times \operatorname{curl}_{\mathbf{x}} \mathbf{A}\right|^{2}-\frac{1}{2}\left|\operatorname{curl}_{\mathbf{x}} \mathbf{A}\right|^{2}\right) d x^{0} d x^{1} d x^{2} d x^{3} \\
-\iiint \int_{\mathbb{R}^{4}}\left(\Psi \rho\left(x^{0}, x^{1}, x^{2}, x^{3}\right)-\mathbf{A} \cdot \mathbf{j}\left(x^{0}, x^{1}, x^{2}, x^{3}\right)\right) d x^{0} d x^{1} d x^{2} d x^{3}+\iiint \int_{\mathbb{R}^{4}} \frac{1}{4 \pi G}\{ \\
\left.\frac{1}{2}\left|\operatorname{curl}_{\mathbf{x}}(\mathbf{v}-\mathbf{r})\right|^{2}-\frac{1}{2}\left|-\frac{\partial}{\partial x^{0}}(\mathbf{v}-\mathbf{r})-\nabla_{\mathbf{x}}\left(\frac{1}{2}|\mathbf{v}|^{2}-\frac{1}{2}|\mathbf{r}|^{2}\right)+\mathbf{v} \times \operatorname{curl}_{\mathbf{x}}(\mathbf{v}-\mathbf{r})\right|^{2}\right\} d x^{0} d x^{1} d x^{2} d x^{3} \\
+\sum_{k=1}^{N} \int_{-\infty}^{+\infty}\left\{-m_{k} \mathcal{G}\left(1-\sum_{j=1}^{3}\left(\frac{d \chi_{k}^{j}}{d x^{0}}\left(x^{0}\right)-v^{j}\left(\chi_{k}\left(x^{0}\right)\right)\right)^{2}\right)\right\} d x^{0} .
\end{gathered}
$$

Next by the Euler-Lagrange of (4.71) we have

$$
\left\{\begin{array}{l}
\frac{\delta L_{\text {total }}}{\delta \mathbf{v}}(\mathbf{v},(\Psi,-\mathbf{A}))=0 \\
\frac{\delta L_{\text {total }}}{\delta \mathbf{A}}(\mathbf{v},(\Psi,-\mathbf{A}))=0 \\
\frac{\delta L_{\text {total }}}{\delta \Psi}(\mathbf{v},(\Psi,-\mathbf{A}))=0 .
\end{array}\right.
$$

On the other hand, by Chain rule we have

$$
\begin{aligned}
\frac{\delta L_{\text {total }}}{\delta \mathbf{v}}(\mathbf{v},(\Psi,-\mathbf{A}))= & \frac{\delta L_{1}}{\delta \mathbf{v}}\left(\mathbf{v},\left(\frac{1}{2}\left(|\mathbf{v}|^{2}-|\mathbf{r}|^{2}\right),-(\mathbf{v}-\mathbf{r})\right),(\Psi,-\mathbf{A})\right) \\
+ & \mathbf{v} \frac{\delta L}{\delta \Phi}\left(\mathbf{v},\left(\frac{1}{2}\left(|\mathbf{v}|^{2}-|\mathbf{r}|^{2}\right),-(\mathbf{v}-\mathbf{r})\right),(\Psi,-\mathbf{A})\right) \\
& +\frac{\delta L_{1}}{\delta \mathbf{h}}\left(\mathbf{v},\left(\frac{1}{2}\left(|\mathbf{v}|^{2}-|\mathbf{r}|^{2}\right),-(\mathbf{v}-\mathbf{r})\right),(\Psi,-\mathbf{A})\right) .
\end{aligned}
$$

Moreover,

$$
\left\{\begin{array}{l}
\frac{\delta L_{\text {total }}}{\delta \mathbf{A}}(\mathbf{v},(\Psi,-\mathbf{A}))=\frac{\delta L_{1}}{\delta \mathbf{A}}\left(\mathbf{v},\left(\frac{1}{2}\left(|\mathbf{v}|^{2}-|\mathbf{r}|^{2}\right),-(\mathbf{v}-\mathbf{r})\right),(\Psi,-\mathbf{A})\right) \\
\frac{\delta L_{\text {total }}}{\delta \Psi}(\mathbf{v},(\Psi,-\mathbf{A}))=\frac{\delta L_{1}}{\delta \Psi}\left(\mathbf{v},\left(\frac{1}{2}\left(|\mathbf{v}|^{2}-|\mathbf{r}|^{2}\right),-(\mathbf{v}-\mathbf{r})\right),(\Psi,-\mathbf{A})\right)
\end{array}\right.
$$

So, by (6.24), (6.26) and (6.25) we obtain

$$
\left\{\begin{array}{l}
\frac{\delta L_{1}}{\delta \mathbf{A}}\left(\mathbf{v},\left(\frac{1}{2}\left(|\mathbf{v}|^{2}-|\mathbf{r}|^{2}\right),-(\mathbf{v}-\mathbf{r})\right),(\Psi,-\mathbf{A})\right)=0 \\
\frac{\delta L_{1}}{\delta \Psi}\left(\mathbf{v},\left(\frac{1}{2}\left(|\mathbf{v}|^{2}-|\mathbf{r}|^{2}\right),-(\mathbf{v}-\mathbf{r})\right),(\Psi,-\mathbf{A})\right)=0
\end{array}\right.
$$

and

$$
\begin{aligned}
\frac{\delta L_{1}}{\delta \mathbf{v}}\left(\mathbf{v},\left(\frac{1}{2}\left(|\mathbf{v}|^{2}-|\mathbf{r}|^{2}\right)\right.\right. & ,-(\mathbf{v}-\mathbf{r})),(\Psi,-\mathbf{A})) \\
+ & \mathbf{v} \frac{\delta L_{1}}{\delta \Phi}\left(\mathbf{v},\left(\frac{1}{2}\left(|\mathbf{v}|^{2}-|\mathbf{r}|^{2}\right),-(\mathbf{v}-\mathbf{r})\right),(\Psi,-\mathbf{A})\right) \\
& +\frac{\delta L_{1}}{\delta \mathbf{h}}\left(\mathbf{v},\left(\frac{1}{2}\left(|\mathbf{v}|^{2}-|\mathbf{r}|^{2}\right),-(\mathbf{v}-\mathbf{r})\right),(\Psi,-\mathbf{A})\right)=0 .
\end{aligned}
$$


Moreover, by (6.21), (6.9), (6.10) and (6.16) we have

$$
\begin{gathered}
\frac{\delta L_{1}}{\delta \mathbf{h}}=\frac{1}{4 \pi G} \operatorname{curl}_{\mathbf{x}} \mathbf{Q}-\frac{1}{4 \pi G}\left(\frac{\partial \mathbf{R}}{\partial x^{0}}-\operatorname{curl}_{\mathbf{x}}\{\mathbf{v} \times \mathbf{R}\}\right), \\
\frac{\delta L}{\delta \Phi}=-\frac{1}{4 \pi G}\left(\operatorname{div}_{\mathbf{x}} \mathbf{R}\right) .
\end{gathered}
$$

and

$$
\frac{\delta L_{1}}{\delta \mathbf{v}}=-\left(\mathbf{e}-E \mathbf{v}+\frac{1}{4 \pi} \mathbf{D} \times \mathbf{B}-\frac{1}{4 \pi G} \mathbf{R} \times \mathbf{Q}\right)
$$

where

$$
\left(e^{0}, e^{1}, e^{2}, e^{3}\right):=(E, \mathbf{e}) \quad \text { with } \quad \mathbf{e}=\left(e^{1}, e^{2}, e^{3}\right) \in \mathbb{R}^{3},
$$

and the contravariant four-vector field $\left(e^{0}, e^{1}, e^{2}, e^{3}\right)$ and the scalar field $E$ are given by

$$
\begin{array}{r}
\left(e^{0}, e^{1}, e^{2}, e^{3}\right)\left(x^{0}, x^{1}, x^{2}, x^{3}\right):= \\
\sum_{k=1}^{N} m_{k} 2 \mathcal{G}^{\prime}\left(1-\sum_{j=1}^{3}\left(\frac{d \chi_{k}^{j}}{d x^{0}}\left(x^{0}\right)-v^{j}\left(\chi_{k}\left(x^{0}\right)\right)\right)^{2}\right) \frac{d \chi_{k}^{j}}{d x^{0}}\left(x^{0}\right) \delta\left(x^{1}-\chi_{k}^{1}\left(\chi^{0}\right), \ldots, x^{3}-\chi_{k}^{3}\left(\chi^{0}\right)\right) \\
\forall\left(x^{0}, x^{1}, x^{2}, x^{3}\right) \in \mathbb{R}^{4}, \quad(6 .
\end{array}
$$

and

$$
\begin{array}{r}
E\left(x^{0}, x^{1}, x^{2}, x^{3}\right):= \\
\sum_{k=1}^{N} m_{k} 2 \mathcal{G}^{\prime}\left(1-\sum_{j=1}^{3}\left(\frac{d \chi_{k}^{j}}{d x^{0}}\left(x^{0}\right)-v^{j}\left(\chi_{k}\left(x^{0}\right)\right)\right)^{2}\right) \delta\left(x^{1}-\chi_{k}^{1}\left(\chi^{0}\right), x^{2}-\chi_{k}^{2}\left(\chi^{0}\right), x^{3}-\chi_{k}^{3}\left(\chi^{0}\right)\right) \\
\forall\left(x^{0}, x^{1}, x^{2}, x^{3}\right) \in \mathbb{R}^{4}, \quad(6 .
\end{array}
$$

where $\mathcal{G}^{\prime}$ is the derivative of the function $\mathcal{G}$. Therefore, using (6.29), (6.30) (6.31) in (6.28) we deduce:

$$
\begin{aligned}
& \left(\mathbf{e}-E \mathbf{v}+\frac{1}{4 \pi} \mathbf{D} \times \mathbf{B}-\frac{1}{4 \pi G} \mathbf{R} \times \mathbf{Q}\right)= \\
& \quad \frac{1}{4 \pi G} \operatorname{curl}_{\mathbf{x}} \mathbf{Q}-\frac{1}{4 \pi G}\left(\frac{\partial \mathbf{R}}{\partial x^{0}}-\operatorname{curl}_{\mathbf{x}}\{\mathbf{v} \times \mathbf{R}\}+\left(\operatorname{div}_{\mathbf{x}} \mathbf{R}\right) \mathbf{v}\right) .
\end{aligned}
$$

Moreover, by (6.27) we have

$$
\frac{1}{4 \pi} \operatorname{div}_{\mathbf{x}} \mathbf{D}-\rho=0,
$$

and

$$
\mathbf{j}+\frac{1}{4 \pi} \frac{\partial \mathbf{D}}{\partial x^{0}}-\frac{1}{4 \pi} \operatorname{curl}_{\mathbf{x}} \mathbf{B}-\frac{1}{4 \pi} \operatorname{curl}_{\mathbf{x}}(\mathbf{v} \times \mathbf{D})=\mathbf{j}+\frac{1}{4 \pi} \frac{\partial \mathbf{D}}{\partial x^{0}}-\frac{1}{4 \pi} \operatorname{curl}_{\mathbf{x}} \mathbf{H}=0 .
$$


So, totally we have

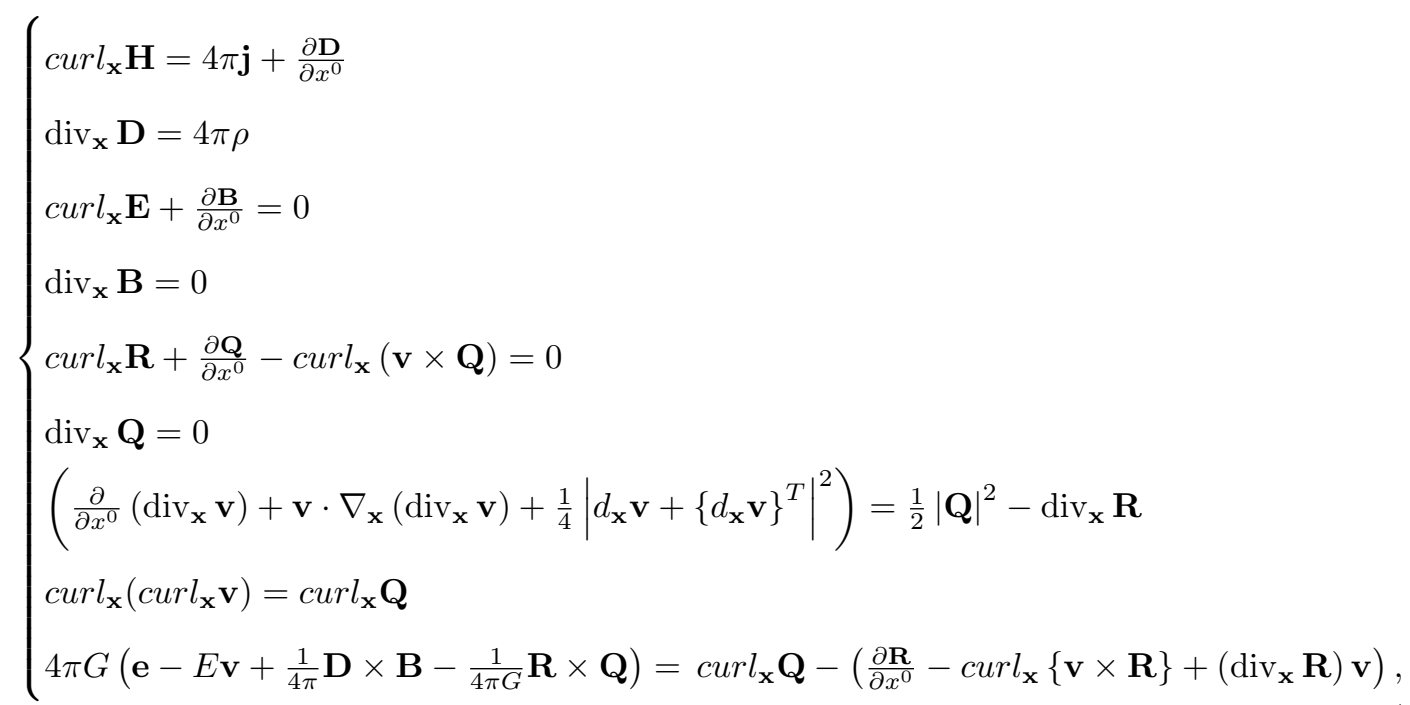

where $\mathbf{D}, \mathbf{B}, \mathbf{E}, \mathbf{H}$ are given by (6.8), so that:

$$
\left\{\begin{array}{l}
\mathbf{B}=\operatorname{curl}_{\mathbf{x}} \mathbf{A} \\
\mathbf{D}=-\nabla_{\mathbf{x}} \Psi-\frac{\partial \mathbf{A}}{\partial x^{0}}+\mathbf{v} \times \operatorname{curl}_{\mathbf{x}} \mathbf{A} \\
\mathbf{E}=-\nabla_{\mathbf{x}} \Psi-\frac{\partial \mathbf{A}}{\partial x^{0}}=\mathbf{D}-\mathbf{v} \times \mathbf{B} \\
\mathbf{H}=\operatorname{curl}_{\mathbf{x}} \mathbf{A}+\mathbf{v} \times\left(-\nabla_{\mathbf{x}} \Psi-\frac{\partial \mathbf{A}}{\partial x^{0}}+\mathbf{v} \times \operatorname{curl}_{\mathbf{x}} \mathbf{A}\right)=\mathbf{B}+\mathbf{v} \times \mathbf{D}
\end{array}\right.
$$

and moreover, by (6.13) in the inertial frame we have:

$$
\left\{\begin{array}{l}
\operatorname{curl}_{\mathbf{x}} \mathbf{H}=4 \pi \mathbf{j}+\frac{\partial \mathbf{D}}{\partial x^{0}} \\
\operatorname{div}_{\mathbf{x}} \mathbf{D}=4 \pi \rho \\
\operatorname{curl}_{\mathbf{x}} \mathbf{E}+\frac{\partial \mathbf{B}}{\partial x^{0}}=0 \\
\operatorname{div}_{\mathbf{x}} \mathbf{B}=0 \\
\mathbf{R}=-\left(\frac{\partial \mathbf{v}}{\partial x^{0}}+d_{\mathbf{x}} \mathbf{v} \cdot \mathbf{v}\right) \\
\mathbf{Q}=\operatorname{curl}_{\mathbf{x}} \mathbf{v} \\
4 \pi G\left(\mathbf{e}-E \mathbf{v}+\frac{1}{4 \pi} \mathbf{D} \times \mathbf{B}-\frac{1}{4 \pi G} \mathbf{R} \times \mathbf{Q}\right)=\operatorname{curl}_{\mathbf{x}} \mathbf{Q}-\left(\frac{\partial \mathbf{R}}{\partial x^{0}}-\operatorname{curl}_{\mathbf{x}}\{\mathbf{v} \times \mathbf{R}\}+\left(\operatorname{div}_{\mathbf{x}} \mathbf{R}\right) \mathbf{v}\right) .
\end{array}\right.
$$

Furthermore, taking $\operatorname{div}_{\mathbf{x}}$ of the both sides of the last equality in (6.38) and using continuum equation (4.50):

$$
\frac{\partial M}{\partial x^{0}}+\operatorname{div}_{\mathbf{x}} \mathbf{j}_{M}=0 \quad \forall\left(x^{0}, x^{1}, x^{2}, x^{3}\right) \in \mathbb{R}^{4},
$$

we deduce

$$
\begin{gathered}
-\left(\frac{\partial M}{\partial x^{0}}+\operatorname{div}_{\mathbf{x}}(M \mathbf{v})\right)+\operatorname{div}_{\mathbf{x}}\left\{\left(\mathbf{e}-\mathbf{j}_{M}\right)-(E-M) \mathbf{v}+\frac{1}{4 \pi} \mathbf{D} \times \mathbf{B}-\frac{1}{4 \pi G} \mathbf{R} \times \mathbf{Q}\right\}= \\
\operatorname{div}_{\mathbf{x}}\left(\mathbf{e}-E \mathbf{v}+\frac{1}{4 \pi} \mathbf{D} \times \mathbf{B}-\frac{1}{4 \pi G} \mathbf{R} \times \mathbf{Q}\right)=-\frac{1}{4 \pi G}\left(\frac{\partial}{\partial x^{0}}\left(\operatorname{div}_{\mathbf{x}} \mathbf{R}\right)+\operatorname{div}_{\mathbf{x}}\left\{\left(\operatorname{div}_{\mathbf{x}} \mathbf{R}\right) \mathbf{v}\right\}\right) .
\end{gathered}
$$


Therefore, considering the proper scalar quantity $Q_{0}$, that we call the field mass, which satisfies

$$
Q_{0}:=-M+\frac{1}{4 \pi G} \operatorname{div}_{\mathbf{x}} \mathbf{R}
$$

by (6.42) we deduce

$$
\frac{\partial Q_{0}}{\partial x^{0}}+\operatorname{div}_{\mathbf{x}}\left(Q_{0} \mathbf{v}\right)=-\operatorname{div}_{\mathbf{x}}\left\{\left(\mathbf{e}-\mathbf{j}_{M}\right)-(E-M) \mathbf{v}+\frac{1}{4 \pi} \mathbf{D} \times \mathbf{B}-\frac{1}{4 \pi G} \mathbf{R} \times \mathbf{Q}\right\} .
$$

Thus, we rewrite (6.38) as:

$$
\left\{\begin{array}{l}
\operatorname{curl}_{\mathbf{x}} \mathbf{H}=4 \pi \mathbf{j}+\frac{\partial \mathbf{D}}{\partial x^{0}} \\
\operatorname{div}_{\mathbf{x}} \mathbf{D}=4 \pi \rho \\
\operatorname{curl}_{\mathbf{x}} \mathbf{E}+\frac{\partial \mathbf{B}}{\partial x^{0}}=0 \\
\operatorname{div}_{\mathbf{x}} \mathbf{B}=0 \\
\operatorname{curl}_{\mathbf{x}} \mathbf{R}+\frac{\partial \mathbf{Q}}{\partial x^{0}}-\operatorname{curl}_{\mathbf{x}}(\mathbf{v} \times \mathbf{Q})=0 \\
\operatorname{div}_{\mathbf{x}} \mathbf{Q}=0 \\
4 \pi G\left(\mathbf{e}-E \mathbf{v}+\frac{1}{4 \pi} \mathbf{D} \times \mathbf{B}-\frac{1}{4 \pi G} \mathbf{R} \times \mathbf{Q}\right)=\operatorname{curl}_{\mathbf{x}} \mathbf{Q}-\left(\frac{\partial \mathbf{R}}{\partial x^{0}}-\operatorname{curl}_{\mathbf{x}}\{\mathbf{v} \times \mathbf{R}\}+\left(\operatorname{div}_{\mathbf{x}} \mathbf{R}\right) \mathbf{v}\right) \\
\operatorname{div}_{\mathbf{x}} \mathbf{R}=4 \pi G\left(M+Q_{0}\right), \\
\left(\frac{\partial}{\partial x^{0}}\left(\operatorname{div}_{\mathbf{x}} \mathbf{v}\right)+\mathbf{v} \cdot \nabla_{\mathbf{x}}\left(\operatorname{div}_{\mathbf{x}} \mathbf{v}\right)+\frac{1}{4}\left|d_{\mathbf{x}} \mathbf{v}+\left\{d_{\mathbf{x}} \mathbf{v}\right\}^{T}\right|^{2}\right)=\frac{1}{2}|\mathbf{Q}|^{2}-\operatorname{div}_{\mathbf{x}} \mathbf{R} \\
\operatorname{curl}_{\mathbf{x}}\left(\operatorname{curl}_{\mathbf{x}} \mathbf{v}\right)=\operatorname{curl}_{\mathbf{x}} \mathbf{Q} \\
\frac{\partial Q_{0}}{\partial x^{0}}+\operatorname{div}_{\mathbf{x}}\left(Q_{0} \mathbf{v}\right)=-\operatorname{div}_{\mathbf{x}}\left\{\left(\mathbf{e}-\mathbf{j}_{M}\right)-(E-M) \mathbf{v}+\frac{1}{4 \pi} \mathbf{D} \times \mathbf{B}-\frac{1}{4 \pi G} \mathbf{R} \times \mathbf{Q}\right\},
\end{array}\right.
$$

where $\mathbf{D}, \mathbf{B}, \mathbf{E}, \mathbf{H}$ are given by (6.8), so that:

$$
\left\{\begin{array}{l}
\mathbf{B}=\operatorname{curl}_{\mathbf{x}} \mathbf{A} \\
\mathbf{D}=-\nabla_{\mathbf{x}} \Psi-\frac{\partial \mathbf{A}}{\partial x^{0}}+\mathbf{v} \times \operatorname{curl}_{\mathbf{x}} \mathbf{A} \\
\mathbf{E}=-\nabla_{\mathbf{x}} \Psi-\frac{\partial \mathbf{A}}{\partial x^{0}}=\mathbf{D}-\mathbf{v} \times \mathbf{B} \\
\mathbf{H}=\operatorname{curl}_{\mathbf{x}} \mathbf{A}+\mathbf{v} \times\left(-\nabla_{\mathbf{x}} \Psi-\frac{\partial \mathbf{A}}{\partial x^{0}}+\mathbf{v} \times \operatorname{curl}_{\mathbf{x}} \mathbf{A}\right)=\mathbf{B}+\mathbf{v} \times \mathbf{D}
\end{array}\right.
$$


and moreover, we rewrite $(6.40)$ in the inertial frame as:

$$
\left\{\begin{array}{l}
\operatorname{curl}_{\mathbf{x}} \mathbf{H}=4 \pi \mathbf{j}+\frac{\partial \mathbf{D}}{\partial x^{0}} \\
\operatorname{div}_{\mathbf{x}} \mathbf{D}=4 \pi \rho \\
\operatorname{curl}_{\mathbf{x}} \mathbf{E}+\frac{\partial \mathbf{B}}{\partial x^{0}}=0 \\
\operatorname{div}_{\mathbf{x}} \mathbf{B}=0 \\
\mathbf{R}=-\left(\frac{\partial \mathbf{v}}{\partial x^{0}}+d_{\mathbf{x}} \mathbf{v} \cdot \mathbf{v}\right) \\
\mathbf{Q}=\operatorname{curl}_{\mathbf{x}} \mathbf{v} \\
4 \pi G\left(\mathbf{e}-E \mathbf{v}+\frac{1}{4 \pi} \mathbf{D} \times \mathbf{B}-\frac{1}{4 \pi G} \mathbf{R} \times \mathbf{Q}\right)=\operatorname{curl}_{\mathbf{x}} \mathbf{Q}-\left(\frac{\partial \mathbf{R}}{\partial x^{0}}-\operatorname{curl}_{\mathbf{x}}\{\mathbf{v} \times \mathbf{R}\}+\left(\operatorname{div}_{\mathbf{x}} \mathbf{R}\right) \mathbf{v}\right) \\
\operatorname{div}_{\mathbf{x}} \mathbf{R}=4 \pi G\left(M+Q_{0}\right) \\
\frac{\partial Q_{0}}{\partial x^{0}}+\operatorname{div}_{\mathbf{x}}\left(Q_{0} \mathbf{v}\right)=-\operatorname{div}_{\mathbf{x}}\left\{\left(\mathbf{e}-\mathbf{j}_{M}\right)-(E-M) \mathbf{v}+\frac{1}{4 \pi} \mathbf{D} \times \mathbf{B}-\frac{1}{4 \pi G} \mathbf{R} \times \mathbf{Q}\right\}
\end{array}\right.
$$

However, as before, here we consider two cases:

- The case of non-relativistic approximation, where $\mathcal{G}(\tau):=\frac{1}{2}(\tau-1)$.

- The case of relativistic particles, where $\mathcal{G}(\tau):=\sqrt{\tau}-1$.

In the first case we have $2 \mathcal{G}^{\prime}(\tau)=1$. In the second case we have $2 \mathcal{G}^{\prime}(\tau)=\tau^{-\frac{1}{2}}=1+\mathcal{O}(\tau-1)$. In the first non-relativistic case by (6.33) and (6.34) we deduce

$$
\mathbf{e}=\mathbf{j}_{M} \quad \text { and } \quad E=M \quad \forall\left(x^{0}, x^{1}, x^{2}, x^{3}\right) \in \mathbb{R}^{4},
$$

In the second relativistic case (6.48) is satisfied approximately, provided we have

$$
\left(\frac{d \chi_{k}^{j}}{d x^{0}}-v^{j}\left(\chi_{k}\right)\right)^{2} \ll 1 \quad \forall k=1,2, \ldots, N .
$$


Thus, by (6.48) we rewrite (6.45) as:

$$
\left\{\begin{array}{l}
\operatorname{curl}_{\mathbf{x}} \mathbf{H}=4 \pi \mathbf{j}+\frac{\partial \mathbf{D}}{\partial x^{0}} \\
\operatorname{div}_{\mathbf{x}} \mathbf{D}=4 \pi \rho \\
\operatorname{curl}_{\mathbf{x}} \mathbf{E}+\frac{\partial \mathbf{B}}{\partial x^{0}}=0 \\
\operatorname{div}_{\mathbf{x}} \mathbf{B}=0 \\
\operatorname{curl}_{\mathbf{x}} \mathbf{R}+\frac{\partial \mathbf{Q}}{\partial x^{0}}-\operatorname{curl}_{\mathbf{x}}(\mathbf{v} \times \mathbf{Q})=0 \\
\operatorname{div}_{\mathbf{x}} \mathbf{Q}=0 \\
4 \pi G\left(\mathbf{j}_{M}-M \mathbf{v}+\frac{1}{4 \pi} \mathbf{D} \times \mathbf{B}-\frac{1}{4 \pi G} \mathbf{R} \times \mathbf{Q}\right)=\operatorname{curl}_{\mathbf{x}} \mathbf{Q}-\left(\frac{\partial \mathbf{R}}{\partial x^{0}}-\operatorname{curl}_{\mathbf{x}}\{\mathbf{v} \times \mathbf{R}\}+\left(\operatorname{div}_{\mathbf{x}} \mathbf{R}\right) \mathbf{v}\right) \\
\operatorname{div}_{\mathbf{x}} \mathbf{R}=4 \pi G\left(M+Q_{0}\right), \\
\left(\frac{\partial}{\partial x^{0}}\left(\operatorname{div}_{\mathbf{x}} \mathbf{v}\right)+\mathbf{v} \cdot \nabla_{\mathbf{x}}\left(\operatorname{div}_{\mathbf{x}} \mathbf{v}\right)+\frac{1}{4}\left|d_{\mathbf{x}} \mathbf{v}+\left\{d_{\mathbf{x}} \mathbf{v}\right\}^{T}\right|^{2}\right)=\frac{1}{2}|\mathbf{Q}|^{2}-\operatorname{div}_{\mathbf{x}} \mathbf{R} \\
\operatorname{curl}_{\mathbf{x}}\left(\operatorname{curl}_{\mathbf{x}} \mathbf{v}\right)=\operatorname{curl}_{\mathbf{x}} \mathbf{Q} \\
\frac{\partial Q_{0}}{\partial x^{0}}+\operatorname{div}_{\mathbf{x}}\left(Q_{0} \mathbf{v}\right)=-\operatorname{div}_{\mathbf{x}}\left\{\frac{1}{4 \pi} \mathbf{D} \times \mathbf{B}-\frac{1}{4 \pi G} \mathbf{R} \times \mathbf{Q}\right\},
\end{array}\right.
$$

where $\mathbf{D}, \mathbf{B}, \mathbf{E}, \mathbf{H}$ are given by (6.8), so that:

$$
\left\{\begin{array}{l}
\mathbf{B}=\operatorname{curl}_{\mathbf{x}} \mathbf{A} \\
\mathbf{D}=-\nabla_{\mathbf{x}} \Psi-\frac{\partial \mathbf{A}}{\partial x^{0}}+\mathbf{v} \times \operatorname{curl}_{\mathbf{x}} \mathbf{A} \\
\mathbf{E}=-\nabla_{\mathbf{x}} \Psi-\frac{\partial \mathbf{A}}{\partial x^{0}}=\mathbf{D}-\mathbf{v} \times \mathbf{B} \\
\mathbf{H}=\operatorname{curl}_{\mathbf{x}} \mathbf{A}+\mathbf{v} \times\left(-\nabla_{\mathbf{x}} \Psi-\frac{\partial \mathbf{A}}{\partial x^{0}}+\mathbf{v} \times \operatorname{curl}_{\mathbf{x}} \mathbf{A}\right)=\mathbf{B}+\mathbf{v} \times \mathbf{D}
\end{array}\right.
$$

and moreover, we rewrite (6.47) in the inertial frame as:

$$
\left\{\begin{array}{l}
\operatorname{curl}_{\mathbf{x}} \mathbf{H}=4 \pi \mathbf{j}+\frac{\partial \mathbf{D}}{\partial x^{0}} \\
\operatorname{div}_{\mathbf{x}} \mathbf{D}=4 \pi \rho \\
\operatorname{curl}_{\mathbf{x}} \mathbf{E}+\frac{\partial \mathbf{B}}{\partial x^{0}}=0 \\
\operatorname{div}_{\mathbf{x}} \mathbf{B}=0 \\
\mathbf{R}=-\left(\frac{\partial \mathbf{v}}{\partial x^{0}}+d_{\mathbf{x}} \mathbf{v} \cdot \mathbf{v}\right) \\
\mathbf{Q}=\operatorname{curl}_{\mathbf{x}} \mathbf{v} \\
4 \pi G\left(\mathbf{j}_{M}-M \mathbf{v}+\frac{1}{4 \pi} \mathbf{D} \times \mathbf{B}-\frac{1}{4 \pi G} \mathbf{R} \times \mathbf{Q}\right)=\operatorname{curl}_{\mathbf{x}} \mathbf{Q}-\left(\frac{\partial \mathbf{R}}{\partial x^{0}}-\operatorname{curl}_{\mathbf{x}}\{\mathbf{v} \times \mathbf{R}\}+\left(\operatorname{div}_{\mathbf{x}} \mathbf{R}\right) \mathbf{v}\right) \\
\operatorname{div}_{\mathbf{x}} \mathbf{R}=4 \pi G\left(M+Q_{0}\right) \\
\frac{\partial Q_{0}}{\partial x^{0}}+\operatorname{div}_{\mathbf{x}}\left(Q_{0} \mathbf{v}\right)=-\operatorname{div}_{\mathbf{x}}\left\{\frac{1}{4 \pi} \mathbf{D} \times \mathbf{B}-\frac{1}{4 \pi G} \mathbf{R} \times \mathbf{Q}\right\}
\end{array}\right.
$$




\section{$7 \quad$ Gravity field of spherically symmetric massive resting body in a coordinate system which is cartesian and inertial si- multaneously}

Consider some coordinate system which is cartesian and inertial simultaneously. Next consider some resting massive spherically symmetric body of Radius $r_{0}$ with center at the point $\left(x^{1}, x^{2}, x^{3}\right)=$ $(0,0,0)$ for all $x^{0} \in \mathbb{R}$. Assume that the electromagnetical fields are negligible with respect to the inertial mass of the body. Then, we rewrite (6.47) as:

$$
\left\{\begin{array}{l}
\mathbf{R}=-\left(\frac{\partial \mathbf{v}}{\partial x^{0}}+d_{\mathbf{x}} \mathbf{v} \cdot \mathbf{v}\right) \\
\mathbf{Q}=\operatorname{curl}_{\mathbf{x}} \mathbf{v} \\
4 \pi G\left(\mathbf{e}-E \mathbf{v}-\frac{1}{4 \pi G} \mathbf{R} \times \mathbf{Q}\right)=\operatorname{curl}_{\mathbf{x}} \mathbf{Q}-\left(\frac{\partial \mathbf{R}}{\partial x^{0}}-\operatorname{curl}_{\mathbf{x}}\{\mathbf{v} \times \mathbf{R}\}+\left(\operatorname{div}_{\mathbf{x}} \mathbf{R}\right) \mathbf{v}\right) \\
\operatorname{div}_{\mathbf{x}} \mathbf{R}=4 \pi G\left(M+Q_{0}\right) \\
\frac{\partial Q_{0}}{\partial x^{0}}+\operatorname{div}_{\mathbf{x}}\left(Q_{0} \mathbf{v}\right)=-\operatorname{div}_{\mathbf{x}}\left\{\left(\mathbf{e}-\mathbf{j}_{M}\right)-(E-M) \mathbf{v}-\frac{1}{4 \pi G} \mathbf{R} \times \mathbf{Q}\right\}
\end{array}\right.
$$

Moreover, we obviously have

$$
M\left(x^{0}, \mathbf{x}\right)=M_{1}(|\mathbf{x}|) \quad \text { and } \quad \mathbf{u}\left(x^{0}, \mathbf{x}\right)=0 \quad \forall\left(x^{0}, \mathbf{x}\right) \in \mathbb{R}^{4},
$$

where we denote $\left(x^{0}, \mathbf{x}\right):=\left(x^{0}, x^{1}, x^{2}, x^{3}\right) \in \mathbb{R}^{4}$ with $\mathbf{x}:=\left(x^{1}, x^{2}, x^{3}\right) \in \mathbb{R}^{3}, \mathbf{u}$ is a three-dimensional velocity field of every point of the given massive body and $M_{1}:=M_{1}(|\mathbf{x}|)$ is the inertial mass density of the body which is assumed to be a radial function such that

$$
M_{1}(|\mathbf{x}|)=0 \quad \text { if } \quad|\mathbf{x}|>r_{0},
$$

where $r_{0}$ is the radius of the body. In particular we have

$$
E\left(x^{0}, \mathbf{x}\right)=M_{1}(|\mathbf{x}|) 2 \mathcal{G}^{\prime}\left(1-\left|\mathbf{v}\left(x^{0}, \mathbf{x}\right)\right|^{2}\right) \quad \forall\left(x^{0}, \mathbf{x}\right):=\left(x^{0}, x^{1}, x^{2}, x^{3}\right) \in \mathbb{R}^{4} .
$$

Thus we simplify the equations for the Gravity in (7.1) as:

$$
\left\{\begin{array}{l}
\mathbf{R}=-\left(\frac{\partial \mathbf{v}}{\partial x^{0}}+d_{\mathbf{x}} \mathbf{v} \cdot \mathbf{v}\right) \\
\mathbf{Q}=\operatorname{curl}_{\mathbf{x}} \mathbf{v} \\
4 \pi G\left(-M_{1}(|\mathbf{x}|) 2 \mathcal{G}^{\prime}\left(1-|\mathbf{v}|^{2}\right) \mathbf{v}-\frac{1}{4 \pi G} \mathbf{R} \times \mathbf{Q}\right)=\operatorname{curl}_{\mathbf{x}} \mathbf{Q}-\left(\frac{\partial \mathbf{R}}{\partial x^{0}}-\operatorname{curl}_{\mathbf{x}}\{\mathbf{v} \times \mathbf{R}\}+\left(\operatorname{div}_{\mathbf{x}} \mathbf{R}\right) \mathbf{v}\right) . \\
\operatorname{div}_{\mathbf{x}} \mathbf{R}=4 \pi G\left(M+Q_{0}\right) \\
\frac{\partial Q_{0}}{\partial x^{0}}+\operatorname{div}_{\mathbf{x}}\left(Q_{0} \mathbf{v}\right)=-\operatorname{div}_{\mathbf{x}}\left\{-M_{1}\left((|\mathbf{x}|) 2 \mathcal{G}^{\prime}\left(1-|\mathbf{v}|^{2}\right)-1\right) \mathbf{v}-\frac{1}{4 \pi G} \mathbf{R} \times \mathbf{Q}\right\} .
\end{array}\right.
$$


We look for stationary (i.e. $x^{0}$-independent) solutions of (7.5). Thus (7.5) implies:

$$
\left\{\begin{array}{l}
\mathbf{R}=-d_{\mathbf{x}} \mathbf{v} \cdot \mathbf{v} \\
\mathbf{Q}=\operatorname{curl}_{\mathbf{x}} \mathbf{v} \\
4 \pi G\left(-M_{1}(|\mathbf{x}|) 2 \mathcal{G}^{\prime}\left(1-|\mathbf{v}|^{2}\right) \mathbf{v}-\frac{1}{4 \pi G} \mathbf{R} \times \mathbf{Q}\right)=\operatorname{curl}_{\mathbf{x}} \mathbf{Q}-\left(-\operatorname{curl}_{\mathbf{x}}\{\mathbf{v} \times \mathbf{R}\}+\left(\operatorname{div}_{\mathbf{x}} \mathbf{R}\right) \mathbf{v}\right) . \\
\operatorname{div}_{\mathbf{x}} \mathbf{R}=4 \pi G\left(M+Q_{0}\right) \\
\operatorname{div}_{\mathbf{x}}\left(Q_{0} \mathbf{v}\right)=-\operatorname{div}_{\mathbf{x}}\left\{-M_{1}\left((|\mathbf{x}|) 2 \mathcal{G}^{\prime}\left(1-|\mathbf{v}|^{2}\right)-1\right) \mathbf{v}-\frac{1}{4 \pi G} \mathbf{R} \times \mathbf{Q}\right\}
\end{array}\right.
$$

On the other hand, by the symmetry considerations of the problem we look for the solution of (7.6) that satisfies $\mathbf{v}(\mathbf{x})=\nabla_{\mathbf{x}} Z_{0}(|\mathbf{x}|)$ where, again by the symmetry of the problem, the scalar function $Z_{0}(|\mathbf{x}|)$ should be radial. In particular, by (7.6) we obtain

$$
\mathbf{Q}=\operatorname{curl}_{\mathbf{x}} \mathbf{v}=0
$$

and thus we simplify (7.6) as:

$$
\left\{\begin{array}{l}
\mathbf{R}=-d_{\mathbf{x}} \mathbf{v} \cdot \mathbf{v}=-\nabla_{\mathbf{x}}\left(\frac{1}{2}|\mathbf{v}|^{2}\right) \\
\mathbf{Q}=\operatorname{curl}_{\mathbf{x}} \mathbf{v}=0 \\
4 \pi G\left(-M_{1}(|\mathbf{x}|) 2 \mathcal{G}^{\prime}\left(1-|\mathbf{v}|^{2}\right) \mathbf{v}\right)=-\left(-\operatorname{curl}_{\mathbf{x}}\{\mathbf{v} \times \mathbf{R}\}+\left(\operatorname{div}_{\mathbf{x}} \mathbf{R}\right) \mathbf{v}\right) \\
\operatorname{div}_{\mathbf{x}} \mathbf{R}=4 \pi G\left(M+Q_{0}\right) \\
\operatorname{div}_{\mathbf{x}}\left(Q_{0} \mathbf{v}\right)=-\operatorname{div}_{\mathbf{x}}\left\{-M_{1}\left((|\mathbf{x}|) 2 \mathcal{G}^{\prime}\left(1-|\mathbf{v}|^{2}\right)-1\right) \mathbf{v}\right\}
\end{array}\right.
$$

In particular, since $\mathbf{v}=\nabla_{\mathbf{x}} Z_{0}(|\mathbf{x}|)$ and $\mathbf{R}=-\nabla_{\mathbf{x}}\left(\frac{1}{2}|\mathbf{v}|^{2}\right)$ are both gradients of radial functions, we have $\mathbf{v} \times \mathbf{R}=0$ and thus, we further simplify (7.8) as:

$$
\left\{\begin{array}{l}
\mathbf{R}=-\nabla_{\mathbf{x}}\left(\frac{1}{2}|\mathbf{v}|^{2}\right) \\
\mathbf{Q}=\operatorname{curl}_{\mathbf{x}} \mathbf{v}=0 \\
\operatorname{div}_{\mathbf{x}} \mathbf{R}=4 \pi G M_{1}(|\mathbf{x}|) 2 \mathcal{G}^{\prime}\left(1-|\mathbf{v}|^{2}\right) \\
\operatorname{curl}_{\mathbf{x}} \mathbf{R}=0 \\
Q_{0}=M_{1}(|\mathbf{x}|)\left(2 \mathcal{G}^{\prime}\left(1-|\mathbf{v}|^{2}\right)-1\right)
\end{array}\right.
$$

However, (7.9) is equivalent to the following:

$$
\left\{\begin{array}{l}
\Delta_{\mathbf{x}}\left(-\frac{1}{2}|\mathbf{v}|^{2}\right)=4 \pi G M_{1}(|\mathbf{x}|) 2 \mathcal{G}^{\prime}\left(1-|\mathbf{v}|^{2}\right) \\
\operatorname{curl}_{\mathbf{x}} \mathbf{v}=0 \\
\mathbf{R}=-\nabla_{\mathbf{x}}\left(\frac{1}{2}|\mathbf{v}|^{2}\right) \\
\mathbf{Q}=0 \\
Q_{0}=M_{1}(|\mathbf{x}|)\left(2 \mathcal{G}^{\prime}\left(1-|\mathbf{v}|^{2}\right)-1\right) .
\end{array}\right.
$$


Therefore, denoting

$$
\Phi_{1}:=-\frac{1}{2}|\mathbf{v}|^{2}
$$

we rewrite (7.10) as:

$$
\left\{\begin{array}{l}
\Delta_{\mathbf{x}} \Phi_{1}=4 \pi G M_{1}(|\mathbf{x}|) 2 \mathcal{G}^{\prime}\left(1+2 \Phi_{1}\right) \\
\Phi_{1}=-\frac{1}{2}|\mathbf{v}|^{2} \\
\operatorname{curl}_{\mathbf{x}} \mathbf{v}=0 \\
\mathbf{R}=-\nabla_{\mathbf{x}}\left(\frac{1}{2}|\mathbf{v}|^{2}\right) \\
\mathbf{Q}=0 \\
Q_{0}=M_{1}(|\mathbf{x}|)\left(2 \mathcal{G}^{\prime}\left(1+2 \Phi_{1}\right)-1\right)
\end{array}\right.
$$

where the scalar field $\Phi_{1}=\Phi(|\mathbf{x}|)$ is radial, and thus, outside of the Massive body surface, where $|\mathbf{x}|>r_{0}$ it coincides with the following Newtonian potential of the massive body:

$$
\Phi_{1}(|\mathbf{x}|)=-\frac{G M_{0}}{|\mathbf{x}|}
$$

where $M_{0}$ is the total effective gravitational mass of the massive body, defined as

$$
M_{0}=\iiint_{|\mathbf{x}| \leq r_{0}} M_{1}(|\mathbf{x}|) 2 \mathcal{G}^{\prime}\left(1+2 \Phi_{1}(|\mathbf{x}|)\right) d \mathbf{x}=\iiint_{|\mathbf{x}| \leq r_{0}} M_{1}(|\mathbf{x}|) 2 \mathcal{G}^{\prime}\left(1-\left|\nabla_{\mathbf{x}} Z_{0}(|\mathbf{x}|)\right|^{2}\right) d \mathbf{x}
$$

Note that, for the inertial mass of the Earth $m_{0}$ we have $m_{0}=\iiint_{|\mathbf{x}| \leq r_{0}} M_{1}(|\mathbf{x}|) d \mathbf{x}$ and thus, in the non-relativistic case, where $2 \mathcal{G}^{\prime}(\tau)=1$ we have $M_{0}=m_{0}$. On the other hand in the relativistic case, where $2 \mathcal{G}^{\prime}(\tau)=(\tau)^{-\frac{1}{2}}>1$ with $\tau<1$ we have $M_{0}>m_{0}$. Next, since there exists a scalar radial field $Z_{0}(|\mathbf{x}|)$ such that $\mathbf{v}(\mathbf{x})=\nabla_{\mathbf{x}} Z_{0}(|\mathbf{x}|)$, by $(7.12)$ we obtain

$$
\left|\frac{d Z_{0}}{d(|\mathbf{x}|)}(|\mathbf{x}|)\right|=\sqrt{-2 \Phi_{1}(\mathbf{x})}
$$

that implies either

$$
\mathbf{v}(\mathbf{x})=\frac{\sqrt{-2 \Phi_{1}(|\mathbf{x}|)}}{|\mathbf{x}|} \mathbf{x}
$$

or

$$
\mathbf{v}(\mathbf{x})=-\frac{\sqrt{-2 \Phi_{1}(|\mathbf{x}|)}}{|\mathbf{x}|} \mathbf{x} .
$$

In particular, on the Earth surface we have:

$$
|\mathbf{v}|=\sqrt{\frac{2 G M_{0}}{r_{0}}},
$$

where $r_{0}$ is the massive body radius and $M_{0}$ is the total effective gravitational mass of the massive body, i.e. the absolute value of the three-dimensional vectorial gravitational potential on a planet surface equals to the escape velocity and its direction is normal to the planet, either downward or upward. 


\subsection{Certain curvilinear coordinate system in the case of stationary radi- ally symmetric gravitational field and relation to the Schwarzschild metric}

Assume that for a given part of the space-time $\mathcal{V} \subset \mathbb{R}^{4}$ in some inertial or non-inertial cartesian coordinate system $(*)$ the gravitational field is stationary and radially symmetric that means that, $\left\{K^{m n}\right\}_{n, m=0,1,2,3}$ is given by (4.29) and $\left\{K_{m n}\right\}_{n, m=0,1,2,3}$ be given by (4.30), so that

$$
\left\{\begin{array}{l}
K^{00}=1 \\
K^{j m}=-\delta_{j m}+v^{j} v^{m} \quad \forall 1 \leq j, m \leq 3 \\
K^{0 j}=K^{j 0}=v^{j} \quad \forall 1 \leq j \leq 3,
\end{array}\right.
$$

and

$$
\left\{\begin{array}{l}
K_{00}=1-|\mathbf{v}|^{2} \\
K_{j m}=-\delta_{j m} \quad \forall 1 \leq j, m \leq 3 \\
K_{0 j}=K_{j 0}=v^{j} \quad \forall 1 \leq j \leq 3
\end{array}\right.
$$

where we denote

$$
\left(v^{0}, v^{1}, v^{2}, v^{3}\right):=(1, \mathbf{v}) \quad \text { with } \quad \mathbf{v}:=\left(v^{1}, v^{2}, v^{3}\right) \in \mathbb{R}^{3},
$$

and the three-dimensional vectorial gravitational potential $\mathbf{v}=\left(v_{1}, v_{2}, v_{3}\right)$ is independent on variable $x^{0}$ and having the form

$$
\mathbf{v}(\mathbf{x})=g(|\mathbf{x}|) \frac{\mathbf{x}}{|\mathbf{x}|} \quad \forall \mathbf{x}
$$

for some scalar function $g(\tau): \mathbb{R} \rightarrow \mathbb{R}$ with

$$
\left(x^{0}, x^{1}, x^{2}, x^{3}\right):=\left(x^{0}, \mathbf{x}\right) \in \mathcal{V} \quad \text { where } \quad \mathbf{x}:=\left(x^{1}, x^{2}, x^{3}\right) \in \mathbb{R}^{3} .
$$

Next, given some differentiable function $F(\mathbf{x}): \mathbb{R}^{3} \rightarrow \mathbb{R}$, consider the change of variables in the four-dimensional space-time $\mathbb{R}^{4}$ :

$$
\left\{\begin{array}{l}
x^{\prime 0}=x^{0}+F\left(x^{1}, x^{2}, x^{3}\right) \\
x^{\prime j}=x^{j} \quad \forall j=1,2,3,
\end{array}\right.
$$

that transforms the cartesian coordinate system $(*)$ to the curvilinear coordinate system $(* *)$ in the four-dimensional space-time $\mathbb{R}^{4}$. Then in the terms of the three-dimensional space we rewrite (7.24) as:

$$
\left\{\begin{array}{l}
x^{\prime 0}=x^{0}+F(\mathbf{x}) \\
\mathbf{x}^{\prime}=\mathbf{x} .
\end{array}\right.
$$

Next if we define a matrix

$$
A=\left\{a_{j}^{i}\right\}_{0 \leq i, j \leq 3}:=\left\{\frac{\partial x^{\prime i}}{\partial x^{j}}\right\}_{0 \leq i, j \leq 3} \in \mathbb{R}^{4 \times 4},
$$


then

$$
\left\{\begin{array}{l}
a_{0}^{0}=1 \\
a_{n}^{m}=\delta_{m n} \quad \forall 1 \leq m, n \leq 3 \\
a_{j}^{0}=\frac{\partial F}{\partial x^{j}} \quad \forall 1 \leq j \leq 3 \\
a_{0}^{j}=0 \quad \forall 1 \leq j \leq 3 .
\end{array}\right.
$$

Next, remind that the contravariant pseudo-metric tensor $\left\{K^{m n}\right\}_{0 \leq m, n \leq 3}$ due to (7.19) has the form of

$$
\left\{\begin{array}{l}
K^{00}=1 \\
K^{j m}=-\delta_{j m}+v^{j} v^{m} \quad \forall 1 \leq j, m \leq 3 \\
K^{0 j}=K^{j 0}=v^{j} \quad \forall 1 \leq j \leq 3,
\end{array}\right.
$$

in the cartesian coordinate system $(*)$. We would like to find the form $\left\{K^{\prime m n}\right\}_{0 \leq m, n \leq 3}$ of this tensor in the curvilinear coordinate system $(* *)$. Then by (10.6) and (7.26) we have:

$$
K^{\prime m n}=\sum_{k=0}^{3} \sum_{j=0}^{3}\left(a_{k}^{m} K^{k j} a_{j}^{n}\right)=\sum_{k=0}^{3} a_{k}^{m}\left(\sum_{j=0}^{3} K^{k j} a_{j}^{n}\right) \quad \forall 0 \leq m, n \leq 3 .
$$

In other words

$$
K^{\prime m n}=a_{0}^{m}\left(K^{00} a_{0}^{n}+\sum_{j=1}^{3} K^{0 j} a_{j}^{n}\right)+\sum_{k=1}^{3} a_{k}^{m}\left(K^{k 0} a_{0}^{n}+\sum_{j=1}^{3} K^{k j} a_{j}^{n}\right) \quad \forall 0 \leq m, n \leq 3 .
$$

In particular, by (7.27) and (7.30) together with (7.28) we obtain:

$$
\begin{aligned}
& K^{\prime 00}= a_{0}^{0}\left(K^{00} a_{0}^{0}+\sum_{j=1}^{3} K^{0 j} a_{j}^{0}\right)+\sum_{k=1}^{3} a_{k}^{0}\left(K^{k 0} a_{0}^{0}+\sum_{j=1}^{3} K^{k j} a_{j}^{0}\right) \\
&=a_{0}^{0}\left(a_{0}^{0}+\sum_{j=1}^{3} 2 v^{j} a_{j}^{0}\right)+\left(\sum_{j=1}^{3} a_{j}^{0} v^{j}\right)^{2}-\sum_{j=1}^{3}\left(a_{j}^{0}\right)^{2}=\left(a_{0}^{0}+\sum_{j=1}^{3} v^{j} a_{j}^{0}\right)^{2}-\sum_{j=1}^{3}\left(a_{j}^{0}\right)^{2}, \\
& K^{\prime 0 n}=K^{\prime n 0}=a_{0}^{0}\left(g^{00} a_{0}^{n}+\sum_{j=1}^{3} K^{0 j} a_{j}^{n}\right)+\sum_{k=1}^{3} a_{k}^{0}\left(K^{k 0} a_{0}^{n}+\sum_{j=1}^{3} K^{k j} a_{j}^{n}\right) \quad \forall 1 \leq n \leq 3, \\
&=a_{0}^{0} v^{n}-a_{n}^{0}+\sum_{k=1}^{3} a_{k}^{0} v^{k} v^{n} \quad \forall 1 \leq n
\end{aligned}
$$

and

$$
\begin{array}{r}
K^{\prime m n}=a_{0}^{m}\left(K^{00} a_{0}^{n}+\sum_{j=1}^{3} K^{0 j} a_{j}^{n}\right)+\sum_{k=1}^{3} a_{k}^{m}\left(K^{k 0} a_{0}^{n}+\sum_{j=1}^{3} K^{k j} a_{j}^{n}\right)=v^{m} v^{n}-\delta_{m n} \\
\forall 1 \leq m, n \leq 3 .
\end{array}
$$


Thus by the last three equations together with (7.27) we deduce:

$$
\left\{\begin{array}{l}
K^{\prime 00}=\left(1+\sum_{j=1}^{3} v^{j} \frac{\partial F}{\partial x^{j}}\right)^{2}-\sum_{j=1}^{3}\left(\frac{\partial F}{\partial x^{j}}\right)^{2} \\
K^{\prime 0 n}=K^{\prime n 0}=v^{n}\left(1+\sum_{j=1}^{3} v^{j} \frac{\partial F}{\partial x^{j}}\right)-\frac{\partial F}{\partial x^{n}} \quad \forall 1 \leq n \leq 3, \\
K^{\prime m n}=v^{m} v^{n}-\delta_{m n} \quad \forall 1 \leq m, n \leq 3 .
\end{array}\right.
$$

Next if $\mathbf{v}$ satisfies (7.22) then choosing the function $F$ to be defined as:

$$
F(\mathbf{x})=\xi(|\mathbf{x}|) \quad \forall \mathbf{x}, \quad \text { where } \quad \frac{d \xi}{d \tau}(\tau)=\frac{g(\tau)}{1-g^{2}(\tau)} \quad \forall \tau
$$

we find that:

$$
v_{n}=\left(1+\sum_{j=1}^{3} v^{j} \frac{\partial F}{\partial x^{j}}\right)^{-1} \quad \frac{\partial F}{\partial x^{n}} \quad \forall 1 \leq n \leq 3,
$$

in other words

$$
v_{n}\left(1+\sum_{j=1}^{3} v^{j} \frac{\partial F}{\partial x^{j}}\right)=\frac{\partial F}{\partial x^{n}} \quad \forall 1 \leq n \leq 3 .
$$

Then we rewrite (7.34) as:

$$
\left\{\begin{array}{l}
K^{\prime 00}=\left(1-|\mathbf{v}|^{2}\right)\left(1+\sum_{j=1}^{3} v^{j} \frac{\partial F}{\partial x^{j}}\right)^{2} \\
K^{\prime 0 n}=K^{\prime n 0}=0 \quad \forall 1 \leq n \leq 3 \\
K^{\prime m n}=v^{m} v^{n}-\delta_{m n} \quad \forall 1 \leq m, n \leq 3 .
\end{array}\right.
$$

On the other hand by (7.37) we have

$$
|\mathbf{v}|^{2}=\left(1-|\mathbf{v}|^{2}\right)\left(\sum_{j=1}^{3} v^{j} \frac{\partial F}{\partial x^{j}}\right) .
$$

We rewrite (7.39) as:

$$
1=\left(1-|\mathbf{v}|^{2}\right)\left(1+\sum_{j=1}^{3} v^{j} \frac{\partial F}{\partial x^{j}}\right)
$$

Therefore, by (7.38) and (7.40) we deduce:

$$
\left\{\begin{array}{l}
K^{\prime 00}=\left(1-|\mathbf{v}|^{2}\right)^{-1}, \\
K^{\prime 0 n}=K^{\prime n 0}=0 \quad \forall 1 \leq n \leq 3, \\
K^{\prime m n}=v^{m} v^{n}-\delta_{m n} \quad \forall 1 \leq m, n \leq 3
\end{array}\right.
$$

Next we find that the covariant pseudo-metric tensor $\left\{K_{m n}^{\prime}\right\}_{0 \leq m, n \leq 3}$ in the curvilinear coordinate system $(* *)$ has the following form:

$$
\left\{\begin{array}{l}
K_{00}^{\prime}=\left(1-|\mathbf{v}|^{2}\right), \\
K_{0 n}^{\prime}=K_{n 0}^{\prime}=0 \quad \forall 1 \leq n \leq 3, \\
K_{m n}^{\prime}=-\left(\left(1-|\mathbf{v}|^{2}\right)^{-1} v^{m} v^{n}+\delta_{m n}\right) \quad \forall 1 \leq m, n \leq 3 .
\end{array}\right.
$$


Indeed, if $\left\{K_{m n}^{\prime}\right\}_{0 \leq m, n \leq 3}$ is defined by (7.42), then by (7.41) we have:

$$
\begin{gathered}
\sum_{k=0}^{3} K_{0 k}^{\prime} K^{\prime k 0}=K_{00}^{\prime} K^{\prime 00}+\sum_{k=1}^{3} K_{0 k}^{\prime} K^{\prime k 0}=1, \\
\sum_{k=0}^{3} K_{m k}^{\prime} K^{\prime k j}=K_{i 0}^{\prime} K^{\prime 0 j}+\sum_{k=1}^{3} K_{m k}^{\prime} K^{\prime k j}=\sum_{k=1}^{3}\left(\left(1-|\mathbf{v}|^{2}\right)^{-1} v^{m} v^{k}+\delta_{m k}\right)\left(\delta_{k j}-v^{k} v^{j}\right) \\
=\delta_{m j}-\left(1-|\mathbf{v}|^{2}\right)^{-1}|\mathbf{v}|^{2} v^{m} v^{j}-v^{m} v^{j}+\left(1-|\mathbf{v}|^{2}\right)^{-1} v^{m} v^{j}=\delta_{m j} \quad \forall 1 \leq m, j \leq 3,
\end{gathered}
$$

and

$$
\begin{array}{cc}
\sum_{k=0}^{3} K_{m k}^{\prime} K^{\prime k 0}=K_{m 0}^{\prime} K^{\prime 00}+\sum_{k=1}^{3} K_{m k}^{\prime} K^{\prime k 0}=0 & \forall 1 \leq m \leq 3 \\
\sum_{k=0}^{3} K_{0 k}^{\prime} K^{\prime k j}=K_{00}^{\prime} K^{\prime 0 j}+\sum_{k=1}^{3} K_{0 k}^{\prime} K^{\prime k j}=0 & \forall 1 \leq j \leq 3 .
\end{array}
$$

So

$$
\sum_{k=0}^{3} K_{m k}^{\prime} K^{\prime k j}=\delta_{m j} \quad \forall 0 \leq m, j \leq 3,
$$

and thus equalities (7.42) indeed define the inverse to $\left\{K^{m n}\right\}_{m, n=0,1,2,3}$ matrix. So by (7.42) we have:

$$
\left\{\begin{array}{l}
K_{00}^{\prime}=\left(1-|\mathbf{v}|^{2}\right), \\
K_{0 n}^{\prime}=K_{n 0}^{\prime}=0 \quad \forall 1 \leq n \leq 3, \\
K_{m n}^{\prime}=-\left(\left(1-|\mathbf{v}|^{2}\right)^{-1} v^{m} v^{n}+\delta_{m n}\right) \quad \forall 1 \leq m, n \leq 3 .
\end{array}\right.
$$

In particular, the quadratic form, induced by the covariant pseudo-metric tensor $\left\{K_{m n}^{\prime}\right\}_{0 \leq m, n \leq 3}$ in the curvilinear coordinate system $(* *)$, that defined on the tangent vectors $\left(d x^{\prime 0}, d x^{\prime 1}, d x^{\prime 2}, d x^{\prime 3}\right) \in$ $\mathbb{R}^{4}$ where $d \mathbf{x}^{\prime}:=\left(d x^{\prime 1}, d x^{\prime 2}, d x^{\prime 3}\right)$ has the following form:

$$
\begin{aligned}
& \sum_{m=0}^{3} \sum_{n=0}^{3} K_{m n}^{\prime} d x^{\prime m} d x^{\prime n}=\left(1-|\mathbf{v}|^{2}\right)\left(d x_{0}^{\prime}\right)^{2}-\left(\left|d \mathbf{x}^{\prime}\right|^{2}+\left(1-|\mathbf{v}|^{2}\right)^{-1}\left|\mathbf{v} \cdot d \mathbf{x}^{\prime}\right|^{2}\right)= \\
& \left(1-|\mathbf{v}|^{2}\right)\left(d x_{0}^{\prime}\right)^{2}-\left(\left(\left|d \mathbf{x}^{\prime}\right|^{2}-\left|\frac{\mathbf{v}}{|\mathbf{v}|} \cdot d \mathbf{x}^{\prime}\right|^{2}\right)+\left(1-|\mathbf{v}|^{2}\right)^{-1}|\mathbf{v}|^{2}\left|\frac{\mathbf{v}}{|\mathbf{v}|} \cdot d \mathbf{x}^{\prime}\right|^{2}+\left|\frac{\mathbf{v}}{|\mathbf{v}|} \cdot d \mathbf{x}^{\prime}\right|^{2}\right) \\
& =\left(1-|\mathbf{v}|^{2}\right)\left(d x_{0}^{\prime}\right)^{2}-\left(\left(1-|\mathbf{v}|^{2}\right)^{-1}\left|\frac{\mathbf{v}}{|\mathbf{v}|} \cdot d \mathbf{x}^{\prime}\right|^{2}+\left(\left|d \mathbf{x}^{\prime}\right|^{2}-\left|\frac{\mathbf{v}}{|\mathbf{v}|} \cdot d \mathbf{x}^{\prime}\right|^{2}\right)\right)
\end{aligned}
$$

Thus taking into account (7.25) and (7.22) we rewrite (7.44) as:

$$
\begin{aligned}
& \sum_{m=0}^{3} \sum_{n=0}^{3} K_{m n}^{\prime} d x^{\prime m} d x^{\prime n}= \\
& \quad\left(1-\left|\mathbf{v}\left(\mathbf{x}^{\prime}\right)\right|^{2}\right)\left(d x_{0}^{\prime}\right)^{2}-\left(\left(1-\left|\mathbf{v}\left(\mathbf{x}^{\prime}\right)\right|^{2}\right)^{-1}\left|\frac{\mathbf{x}^{\prime}}{\left|\mathbf{x}^{\prime}\right|} \cdot d \mathbf{x}^{\prime}\right|^{2}+\left(\left|d \mathbf{x}^{\prime}\right|^{2}-\left|\frac{\mathbf{x}^{\prime}}{\left|\mathbf{x}^{\prime}\right|} \cdot d \mathbf{x}^{\prime}\right|^{2}\right)\right) .
\end{aligned}
$$

Next, up to the end of this subsection, assume that our cartesian coordinate system $(*)$ is inertial and cartesian simultaneously and our gravitational field is formed by the resting spherical symmetric 
massive body of the effective gravitational mass $M_{0}$ and radius $r_{0}$ like the Earth, the Sun et.al. with the center at the point $\left(x^{1}, x^{2}, x^{3}\right)=0$. Then, as we get before, we have either (7.16):

$$
\mathbf{v}(\mathbf{x})=\frac{\sqrt{-2 \Phi_{1}(|\mathbf{x}|)}}{|\mathbf{x}|} \mathbf{x},
$$

or (7.17):

$$
\mathbf{v}(\mathbf{x})=-\frac{\sqrt{-2 \Phi_{1}(|\mathbf{x}|)}}{|\mathbf{x}|} \mathbf{x},
$$

where outside of the massive body surface we have

$$
\Phi_{1}(|\mathbf{x}|)=-\frac{G M_{0}}{|\mathbf{x}|},
$$

with the total effective gravitational mass of the massive body $M_{0}$, defined as in (7.14) by

$$
M_{0}=\iiint_{|\mathbf{x}| \leq r_{0}} M_{1}(|\mathbf{x}|) 2 \mathcal{G}^{\prime}\left(1+2 \Phi_{1}(|\mathbf{x}|)\right) d \mathbf{x}=\iiint_{|\mathbf{x}| \leq r_{0}} M_{1}(|\mathbf{x}|) 2 \mathcal{G}^{\prime}\left(1-\left|\nabla_{\mathbf{x}} Z_{0}(|\mathbf{x}|)\right|^{2}\right) d \mathbf{x} .
$$

Thus in particular,

$$
|\mathbf{v}(\mathbf{x})|^{2}=-2 \Phi_{1}(|\mathbf{x}|)
$$

and outside of the massive body surface we have:

$$
|\mathbf{v}(\mathbf{x})|^{2}=\frac{2 G M_{0}}{|\mathbf{x}|} .
$$

Both (7.46) and (7.47) are particular cases of (7.22), with

$$
g(\tau)= \pm \sqrt{-2 \Phi_{1}(\tau)}
$$

and in particular, outside of the massive body surface we have:

$$
g(|x|)= \pm \sqrt{\frac{2 G M_{0}}{|\mathbf{x}|}}
$$

Thus defining the function $F(\mathbf{x})$ as in (7.35), that always can be done in the case $\frac{2 G M_{0}}{r_{0}}<1$, we can define the change of variables from coordinate system $(*)$ to the curvilinear coordinate system $(* *)$ in the four-dimensional space-time $\mathbb{R}^{4}$ as in (7.25):

$$
\left\{\begin{array}{l}
x^{\prime 0}=x^{0}+F(\mathbf{x}) \\
\mathbf{x}^{\prime}=\mathbf{x} .
\end{array}\right.
$$

Then by inserting (7.46) or (7.47) into (7.43) we deduce the form of the covariant pseudo-metric tensor in the curvilinear coordinate system $(* *)$ :

$$
\left\{\begin{array}{l}
K_{00}^{\prime}=\left(1+2 \Phi_{1}\left(\left|\mathbf{x}^{\prime}\right|\right)\right), \\
K_{0 n}^{\prime}=K_{n 0}^{\prime}=0 \quad \forall 1 \leq n \leq 3, \\
K_{m n}^{\prime}=\left(\left(1+2 \Phi_{1}\left(\left|\mathbf{x}^{\prime}\right|\right)\right)^{-1} 2 \Phi_{1}\left(\left|\mathbf{x}^{\prime}\right|\right) \frac{x_{m}^{\prime}}{\left|\mathbf{x}^{\prime}\right|} \frac{x_{n}^{\prime}}{\left|\mathbf{x}^{\prime}\right|}-\delta_{m n}\right) \quad \forall 1 \leq m, n \leq 3 .
\end{array}\right.
$$


Moreover, by (7.45) we have:

$$
\begin{aligned}
& \sum_{m=0}^{3} \sum_{n=0}^{3} K_{m n}^{\prime} d x^{\prime m} d x^{\prime n}= \\
& \quad\left(1+2 \Phi_{1}\left(\left|\mathbf{x}^{\prime}\right|\right)\right) d x_{0}^{\prime 2}-\left(\left(1+2 \Phi_{1}\left(\left|\mathbf{x}^{\prime}\right|\right)\right)^{-1}\left|\frac{\mathbf{x}^{\prime}}{\left|\mathbf{x}^{\prime}\right|} \cdot d \mathbf{x}^{\prime}\right|^{2}+\left(\left|d \mathbf{x}^{\prime}\right|^{2}-\left|\frac{\mathbf{x}^{\prime}}{\left|\mathbf{x}^{\prime}\right|} \cdot d \mathbf{x}^{\prime}\right|^{2}\right)\right) .
\end{aligned}
$$

In particular, outside of the massive body surface, i.e. when $\left|x^{\prime}\right| \geq r_{0}$ we rewrite (7.55) and (7.56) as:

$$
\left\{\begin{array}{l}
K_{00}^{\prime}=\left(1-\frac{2 G M_{0}}{\left|\mathbf{x}^{\prime}\right|}\right), \\
K_{0 n}^{\prime}=K_{n 0}^{\prime}=0 \quad \forall 1 \leq n \leq 3, \\
K_{m n}^{\prime}=-\left(\left(1-\frac{2 G M_{0}}{\left|\mathbf{x}^{\prime}\right|}\right)^{-1} \frac{2 G M_{0}}{\left|\mathbf{x}^{\prime}\right|} \frac{x_{m}^{\prime}}{\left|\mathbf{x}^{\prime}\right|} \mid \frac{x_{n}^{\prime}}{\left|\mathbf{x}^{\prime}\right|}+\delta_{m n}\right) \quad \forall 1 \leq m, n \leq 3,
\end{array}\right.
$$

and

$$
\begin{aligned}
& \sum_{m=0}^{3} \sum_{n=0}^{3} K_{m n}^{\prime} d x^{\prime m} d x^{\prime n}= \\
& \quad\left(1-\frac{2 G M_{0}}{\left|\mathbf{x}^{\prime}\right|}\right) d x_{0}^{\prime 2}-\left(\left(1-\frac{2 G M_{0}}{\left|\mathbf{x}^{\prime}\right|}\right)^{-1}\left|\frac{\mathbf{x}^{\prime}}{\left|\mathbf{x}^{\prime}\right|} \cdot d \mathbf{x}^{\prime}\right|^{2}+\left(\left|d \mathbf{x}^{\prime}\right|^{2}-\left|\frac{\mathbf{x}^{\prime}}{\left|\mathbf{x}^{\prime}\right|} \cdot d \mathbf{x}^{\prime}\right|^{2}\right)\right) .
\end{aligned}
$$

Therefore, we get that in coordinate system $(* *)$, outside of the massive body, the covariant pseudometric tensor in (7.57) and (7.58) exactly the same as the well known Schwarzschild metric from the General Relativity (see [9], pages 180-181). Indeed in the spherical coordinates in $\mathbb{R}^{3}$ we rewrite (7.58) as:

$$
\begin{aligned}
& \sum_{m=0}^{3} \sum_{n=0}^{3} K_{m n}^{\prime} d x^{\prime m} d x^{\prime n}= \\
& \quad\left(1-\frac{2 G M_{0}}{r^{\prime}}\right) d x_{0}^{\prime 2}-\left(\left(1-\frac{2 G M_{0}}{r^{\prime}}\right)^{-1}\left(d r^{\prime}\right)^{2}+\left(r^{\prime}\right)^{2}\left(\left(d \theta^{\prime}\right)^{2}+\sin ^{2}\left(\theta^{\prime}\right)\left(d \varphi^{\prime}\right)^{2}\right)\right),
\end{aligned}
$$

and this is exactly the classical Schwarzschild metric! ([7],[9])

In particular, all the optical effects that we find in the framework of our model coincide with the effects considered in the framework of General Relativity for the Schwarzschild metric. In particular, the Michelson-Morely experiment and all Sagnac-type effects will lead to the same result in the framework of our model like in the case of the General relativity. Moreover, since the Maxwell equations in both models have the same tensor form, all the electromagnetic effects, where the time does not appear explicitly, will be the same. Similarly, the curvature of the light path in the Sun's gravitational field will be the same in both models. Finally, in the particular case of $\mathcal{G}(\tau)=\sqrt{\tau}$, i.e. in the case of the relativistic Lagrangian of the motion in (2.102) all the mechanical effects will be the same in the framework of our model like in the case of the General relativity for the Schwarzschild metric, provided that the time does not appear explicitly in this effects. In particular, the movement of the Mercury planet in the Sun's gravitational field will be the same in both models. 


\section{Newtonian gravity as an approximation of (6.52).}

We now approximate the Euler-Lagrange in (6.52). First of all, in the usual circumstances we obviously have for the electromagnetic field:

$$
\left|\frac{1}{4 \pi} \mathbf{D} \times \mathbf{B}\right| \ll M .
$$

Thus, by (8.1), we approximate (6.50) as:

$$
\left\{\begin{array}{l}
\operatorname{curl}_{\mathbf{x}} \mathbf{H}=4 \pi \mathbf{j}+\frac{\partial \mathbf{D}}{\partial x^{0}} \\
\operatorname{div}_{\mathbf{x}} \mathbf{D}=4 \pi \rho \\
\operatorname{curl}_{\mathbf{x}} \mathbf{E}+\frac{\partial \mathbf{B}}{\partial x^{0}}=0 \\
\operatorname{div}_{\mathbf{x}} \mathbf{B}=0 \\
\operatorname{curl}_{\mathbf{x}} \mathbf{R}+\frac{\partial \mathbf{Q}}{\partial x^{0}}-\operatorname{curl}_{\mathbf{x}}(\mathbf{v} \times \mathbf{Q})=0 \\
\operatorname{div}_{\mathbf{x}} \mathbf{Q}=0 \\
4 \pi G\left(M \mathbf{u}-M \mathbf{v}-\frac{1}{4 \pi G} \mathbf{R} \times \mathbf{Q}\right)=\operatorname{curl}_{\mathbf{x}} \mathbf{Q}-\left(\frac{\partial \mathbf{R}}{\partial x^{0}}-\operatorname{curl}_{\mathbf{x}}\{\mathbf{v} \times \mathbf{R}\}+\left(\operatorname{div}_{\mathbf{x}} \mathbf{R}\right) \mathbf{v}\right) \\
\operatorname{div}_{\mathbf{x}} \mathbf{R}=4 \pi G\left(M+Q_{0}\right) \\
\left(\frac{\partial}{\partial x^{0}}\left(\operatorname{div}_{\mathbf{x}} \mathbf{v}\right)+\mathbf{v} \cdot \nabla_{\mathbf{x}}\left(\operatorname{div}_{\mathbf{x}} \mathbf{v}\right)+\frac{1}{4}\left|d_{\mathbf{x}} \mathbf{v}+\left\{d_{\mathbf{x}} \mathbf{v}\right\}^{T}\right|^{2}\right)=\frac{1}{2}|\mathbf{Q}|^{2}-\operatorname{div}_{\mathbf{x}} \mathbf{R} \\
\operatorname{curl}_{\mathbf{x}}\left(\operatorname{curl}_{\mathbf{x}} \mathbf{v}\right)=\operatorname{curl}_{\mathbf{x}} \mathbf{Q} \\
\frac{\partial Q_{0}}{\partial x^{0}}+\operatorname{div}_{\mathbf{x}}\left(Q_{0} \mathbf{v}\right)=\operatorname{div}_{\mathbf{x}}\left\{\frac{1}{4 \pi G} \mathbf{R} \times \mathbf{Q}\right\}
\end{array}\right.
$$

where $\mathbf{u}:=\mathbf{u}\left(x^{0}, x^{1}, x^{2}, x^{3}\right)$ is the field of the velocities of the matter so that

$$
\mathbf{j}_{M}\left(x^{0}, x^{1}, x^{2}, x^{3}\right)=M\left(x^{0}, x^{1}, x^{2}, x^{3}\right) \mathbf{u}\left(x^{0}, x^{1}, x^{2}, x^{3}\right) \quad \forall\left(x^{0}, x^{1}, x^{2}, x^{3}\right) \in \mathbb{R}^{4},
$$

and where $\mathbf{D}, \mathbf{B}, \mathbf{E}, \mathbf{H}$ are given by (6.8), so that:

$$
\left\{\begin{array}{l}
\mathbf{B}=\operatorname{curl}_{\mathbf{x}} \mathbf{A} \\
\mathbf{D}=-\nabla_{\mathbf{x}} \Psi-\frac{\partial \mathbf{A}}{\partial x^{0}}+\mathbf{v} \times \operatorname{curl}_{\mathbf{x}} \mathbf{A} \\
\mathbf{E}=-\nabla_{\mathbf{x}} \Psi-\frac{\partial \mathbf{A}}{\partial x^{0}}=\mathbf{D}-\mathbf{v} \times \mathbf{B} \\
\mathbf{H}=\operatorname{curl}_{\mathbf{x}} \mathbf{A}+\mathbf{v} \times\left(-\nabla_{\mathbf{x}} \Psi-\frac{\partial \mathbf{A}}{\partial x^{0}}+\mathbf{v} \times \operatorname{curl}_{\mathbf{x}} \mathbf{A}\right)=\mathbf{B}+\mathbf{v} \times \mathbf{D} .
\end{array}\right.
$$


Furthermore, we approximate (6.52) in the inertial frame as:

$$
\left\{\begin{array}{l}
\operatorname{curl}_{\mathbf{x}} \mathbf{H}=4 \pi \mathbf{j}+\frac{\partial \mathbf{D}}{\partial x^{0}} \\
\operatorname{div}_{\mathbf{x}} \mathbf{D}=4 \pi \rho \\
\operatorname{curl}_{\mathbf{x}} \mathbf{E}+\frac{\partial \mathbf{B}}{\partial x^{0}}=0 \\
\operatorname{div}_{\mathbf{x}} \mathbf{B}=0 \\
\mathbf{R}=-\left(\frac{\partial \mathbf{v}}{\partial x^{0}}+d_{\mathbf{x}} \mathbf{v} \cdot \mathbf{v}\right) \\
\mathbf{Q}=\operatorname{curl}_{\mathbf{x}} \mathbf{v} \\
4 \pi G\left(M \mathbf{u}-M \mathbf{v}-\frac{1}{4 \pi G} \mathbf{R} \times \mathbf{Q}\right)=\operatorname{curl}_{\mathbf{x}} \mathbf{Q}-\left(\frac{\partial \mathbf{R}}{\partial x^{0}}-\operatorname{curl}_{\mathbf{x}}\{\mathbf{v} \times \mathbf{R}\}+\left(\operatorname{div}_{\mathbf{x}} \mathbf{R}\right) \mathbf{v}\right) \\
\operatorname{div}_{\mathbf{x}} \mathbf{R}=4 \pi G\left(M+Q_{0}\right) \\
\frac{\partial Q_{0}}{\partial x^{0}}+\operatorname{div}_{\mathbf{x}}\left(Q_{0} \mathbf{v}\right)=\operatorname{div}_{\mathbf{x}}\left\{\frac{1}{4 \pi G} \mathbf{R} \times \mathbf{Q}\right\}
\end{array}\right.
$$

On the other hand, we can rewrite (8.5) in the inertial frame as:

$$
\left\{\begin{array}{l}
\operatorname{curl}_{\mathbf{x}} \mathbf{H}=4 \pi \mathbf{j}+\frac{\partial \mathbf{D}}{\partial x^{0}} \\
\operatorname{div}_{\mathbf{x}} \mathbf{D}=4 \pi \rho \\
\operatorname{curl}_{\mathbf{x}} \mathbf{E}+\frac{\partial \mathbf{B}}{\partial x^{0}}=0 \\
\operatorname{div}_{\mathbf{x}} \mathbf{B}=0 \\
-\left(\operatorname{div}_{\mathbf{x}}\left\{\frac{\partial \mathbf{v}}{\partial x^{0}}+d_{\mathbf{x}} \mathbf{v} \cdot \mathbf{v}\right\}\right) \mathbf{u}_{\mathbf{v}}+\left(\frac{\partial \mathbf{v}}{\partial x^{0}}+d_{\mathbf{x}} \mathbf{v} \cdot \mathbf{v}\right) \times\left(\operatorname{curl}_{\mathbf{x}} \mathbf{v}\right)=\operatorname{curl}_{\mathbf{x}}\left(\operatorname{curl}_{\mathbf{x}} \mathbf{v}\right) \\
+\frac{\partial}{\partial x^{0}}\left\{\frac{\partial \mathbf{v}}{\partial x^{0}}+d_{\mathbf{x}} \mathbf{v} \cdot \mathbf{v}\right\}-\operatorname{curl}_{\mathbf{x}}\left\{\mathbf{v} \times\left(\frac{\partial \mathbf{v}}{\partial x^{0}}+d_{\mathbf{x}} \mathbf{v} \cdot \mathbf{v}\right)\right\}+4 \pi G Q_{0}\left(\mathbf{u}_{\mathbf{v}}-\mathbf{v}\right) \\
\operatorname{div}_{\mathbf{x}}\left\{\frac{\partial \mathbf{v}}{\partial x^{0}}+d_{\mathbf{x}} \mathbf{v} \cdot \mathbf{v}\right\}=-4 \pi G\left(M+Q_{0}\right) \\
\frac{\partial Q_{0}}{\partial x^{0}}+\operatorname{div}_{\mathbf{x}}\left(Q_{0} \mathbf{v}\right)=-\operatorname{div}_{\mathbf{x}}\left\{\frac{1}{4 \pi G}\left(\frac{\partial \mathbf{v}}{\partial x^{0}}+d_{\mathbf{x}} \mathbf{v} \cdot \mathbf{v}\right) \times\left(\operatorname{curl}_{\mathbf{x}} \mathbf{v}\right)\right\}
\end{array}\right.
$$

where we denote

$$
\mathbf{u}_{\mathbf{v}}:=\left\{\begin{array}{lll}
\mathbf{u} & \text { if } & M \neq 0 \\
\mathbf{v} & \text { if } & M=0 .
\end{array}\right.
$$

Furthermore, we assume the non-relativistic approximation and quasistationery nature of the field $\mathbf{v}$, so that

$$
\begin{aligned}
&\left|\frac{\partial^{2} \mathbf{v}}{\partial\left(x^{0}\right)^{2}}\right| \ll\left|d_{\mathbf{x}}^{2} \mathbf{v}\right|, \quad\left|\frac{\partial \mathbf{v}}{\partial x^{0}}\right|^{2} \ll\left|d_{\mathbf{x}} \mathbf{v}\right|^{2}, \quad\left|d_{\mathbf{x}}\left\{\frac{\partial \mathbf{v}}{\partial x^{0}}\right\}\right|^{2} \ll\left|d_{\mathbf{x}}^{2} \mathbf{v}\right|^{2}, \\
&|\mathbf{u}|^{2} \ll 1 \quad \text { and } \quad|\mathbf{v}|^{2} \ll 1 .
\end{aligned}
$$


Thus, by (8.8) and (8.3) we approximate (8.6) in the inertial frame as as:

$$
\left\{\begin{array}{l}
\operatorname{curl}_{\mathbf{x}} \mathbf{H}=4 \pi \mathbf{j}+\frac{\partial \mathbf{D}}{\partial x^{0}} \\
\operatorname{div}_{\mathbf{x}} \mathbf{D}=4 \pi \rho \\
\operatorname{curl}{ }_{\mathbf{x}} \mathbf{E}+\frac{\partial \mathbf{B}}{\partial x^{0}}=0 \\
\operatorname{div}_{\mathbf{x}} \mathbf{B}=0 \\
\operatorname{div}_{\mathbf{x}}\left\{\frac{\partial \mathbf{v}}{\partial x^{0}}+d_{\mathbf{x}} \mathbf{v} \cdot \mathbf{v}\right\}=-4 \pi G\left(M+Q_{0}\right) \\
\operatorname{curl}_{\mathbf{x}}\left(\operatorname{curl}_{\mathbf{x}} \mathbf{v}\right)+4 \pi G Q_{0}\left(\mathbf{u}_{\mathbf{v}}-\mathbf{v}\right)+\nabla_{x} \zeta=0 \\
\frac{\partial Q_{0}}{\partial x^{0}}+\operatorname{div}_{\mathbf{x}}\left(Q_{0} \mathbf{v}\right)=0 .
\end{array}\right.
$$

where $\zeta$ is some unspecified approximately negligible scalar field. However, the obvious solution of the last two equations in (8.9) are

$$
\left\{\begin{array}{l}
\operatorname{curl}_{\mathbf{x}} \mathbf{v}=0 \\
Q_{0}=0
\end{array}\right.
$$

So, by (8.10) we rewrite (8.9) as:

$$
\left\{\begin{array}{l}
\operatorname{curl}_{\mathbf{x}} \mathbf{H}=4 \pi \mathbf{j}+\frac{\partial \mathbf{D}}{\partial x^{0}} \\
\operatorname{div}_{\mathbf{x}} \mathbf{D}=4 \pi \rho \\
\operatorname{curl}_{\mathbf{x}} \mathbf{E}+\frac{\partial \mathbf{B}}{\partial x^{0}}=0 \\
\operatorname{div}_{\mathbf{x}} \mathbf{B}=0 \\
\operatorname{curl}_{\mathbf{x}} \mathbf{v}=0 \\
\operatorname{div}_{\mathbf{x}}\left\{\frac{\partial \mathbf{v}}{\partial x^{0}}+d_{\mathbf{x}} \mathbf{v} \cdot \mathbf{v}\right\}=-4 \pi G M
\end{array}\right.
$$

We obviously can rewrite (8.11) as:

$$
\left\{\begin{array}{l}
\operatorname{curl}_{\mathbf{x}} \mathbf{H}=4 \pi \mathbf{j}+\frac{\partial \mathbf{D}}{\partial x^{0}} \\
\operatorname{div}_{\mathbf{x}} \mathbf{D}=4 \pi \rho \\
\operatorname{curl}_{\mathbf{x}} \mathbf{E}+\frac{\partial \mathbf{B}}{\partial x^{0}}=0 \\
\operatorname{div}_{\mathbf{x}} \mathbf{B}=0 \\
\operatorname{curl}_{\mathbf{x}} \mathbf{v}=0 \\
\operatorname{div}_{\mathbf{x}}\left\{\frac{\partial \mathbf{v}}{\partial x^{0}}+\nabla_{\mathbf{x}}\left(\frac{1}{2}|\mathbf{v}|^{2}\right)\right\}=-4 \pi G M
\end{array}\right.
$$

where $\mathbf{D}, \mathbf{B}, \mathbf{E}, \mathbf{H}$ are given by (6.8), so that:

$$
\left\{\begin{array}{l}
\mathbf{B}=\operatorname{curl}_{\mathbf{x}} \mathbf{A} \\
\mathbf{D}=-\nabla_{\mathbf{x}} \Psi-\frac{\partial \mathbf{A}}{\partial x^{0}}+\mathbf{v} \times \operatorname{curl}_{\mathbf{x}} \mathbf{A} \\
\mathbf{E}=-\nabla_{\mathbf{x}} \Psi-\frac{\partial \mathbf{A}}{\partial x^{0}}=\mathbf{D}-\mathbf{v} \times \mathbf{B} \\
\mathbf{H}=\operatorname{curl}_{\mathbf{x}} \mathbf{A}+\mathbf{v} \times\left(-\nabla_{\mathbf{x}} \Psi-\frac{\partial \mathbf{A}}{\partial x^{0}}+\mathbf{v} \times \operatorname{curl}_{\mathbf{x}} \mathbf{A}\right)=\mathbf{B}+\mathbf{v} \times \mathbf{D}
\end{array}\right.
$$


and furthermore, we rewrite two last equations in (8.12) as:

$$
\left\{\begin{array}{l}
\mathbf{v}=\nabla_{\mathbf{x}} Z \\
\operatorname{div}_{\mathbf{x}}\left\{\nabla_{\mathbf{x}} \Phi\right\}=4 \pi G M \quad \text { where } \\
\frac{\partial Z}{\partial x^{0}}+\frac{1}{2}\left|\nabla_{\mathbf{x}} Z\right|^{2}=\frac{\partial Z}{\partial x^{0}}+\frac{1}{2}|\mathbf{v}|^{2}=-\Phi,
\end{array}\right.
$$

where $Z$ is some scalar field. So, finally

$$
\left\{\begin{array}{l}
\operatorname{curl}_{\mathbf{x}} \mathbf{v}=0 \quad \text { and } \\
\frac{\partial \mathbf{v}}{\partial x^{0}}+\nabla_{\mathbf{x}}\left(\frac{1}{2}|\mathbf{v}|^{2}\right)=-\nabla_{\mathbf{x}} \Phi \quad \text { where } \\
\operatorname{div}_{\mathbf{x}}\left\{\nabla_{\mathbf{x}} \Phi\right\}=4 \pi G M .
\end{array}\right.
$$

On the other hand, we remind that the motion of the particle with the mass $m$ and the charge $\sigma$ in the gravitational and electromagnetical fields is governed, in the non-relativistic case by equations (5.15) so that

$$
\begin{aligned}
m \frac{d^{2} \mathbf{z}}{d\left(x^{0}\right)^{2}}\left(x^{0}\right)=m\left(\frac{\partial}{\partial x^{0}} \mathbf{v}\left(x^{0}, \mathbf{z}\left(x^{0}\right)\right)+\right. & \left.\nabla_{\mathbf{x}}\left(\frac{1}{2}\left|\mathbf{v}\left(x^{0}, \mathbf{z}\left(x^{0}\right)\right)\right|^{2}\right)-\frac{d \mathbf{z}}{d x^{0}}\left(x^{0}\right) \times \operatorname{curl}_{\mathbf{x}} \mathbf{v}\left(x^{0}, \mathbf{z}\left(x^{0}\right)\right)\right) \\
+ & \sigma\left(\mathbf{E}\left(x^{0}, \mathbf{z}\left(x^{0}\right)\right)+\frac{d \mathbf{z}}{d x^{0}}\left(x^{0}\right) \times \mathbf{B}\left(x^{0}, \mathbf{z}\left(x^{0}\right)\right)\right) .
\end{aligned}
$$

Thus, inserting (8.15) into (8.16) gives that in the coordinate system, which is cartesian and inertial simultaneously, we have

$$
m \frac{d^{2} \mathbf{z}}{d\left(x^{0}\right)^{2}}\left(x^{0}\right)=-m \nabla_{\mathbf{x}} \Phi\left(x^{0}, \mathbf{z}\left(x^{0}\right)\right)+\sigma\left(\mathbf{E}\left(x^{0}, \mathbf{z}\left(x^{0}\right)\right)+\frac{d \mathbf{z}}{d x^{0}}\left(x^{0}\right) \times \mathbf{B}\left(x^{0}, \mathbf{z}\left(x^{0}\right)\right)\right),
$$

where $\Phi$ is given by

$$
\operatorname{div}_{\mathbf{x}}\left\{\nabla_{\mathbf{x}} \Phi\right\}=4 \pi G M .
$$

However, (8.17) with (8.18) is obviously exactly the case of the classical Newtonian Gravity! Thus, in the non-relativistic approximation and in the case of quasistationery gravitational field, the Newtonian gravity is indeed a valid approximation of (6.52), provided we deal with the coordinate system, which is cartesian and inertial simultaneously.

Similarly, if we do not assume anymore that our cartesian coordinate system is inertial, then in the non-relativistic approximation and in the case of quasistationery gravitational field, by (8.8) we approximate (8.2) as:

$$
\left\{\begin{array}{l}
\operatorname{curl}_{\mathbf{x}} \mathbf{H}=4 \pi \mathbf{j}+\frac{\partial \mathbf{D}}{\partial x^{0}} \\
\operatorname{div}_{\mathbf{x}} \mathbf{D}=4 \pi \rho \\
\operatorname{curl}_{\mathbf{x}} \mathbf{E}+\frac{\partial \mathbf{B}}{\partial x^{0}}=0 \\
\operatorname{div}_{\mathbf{x}} \mathbf{B}=0 \\
\left(\frac{\partial}{\partial x^{0}}\left(\operatorname{div}_{\mathbf{x}} \mathbf{v}\right)+\mathbf{v} \cdot \nabla_{\mathbf{x}}\left(\operatorname{div}_{\mathbf{x}} \mathbf{v}\right)+\frac{1}{4}\left|d_{\mathbf{x}} \mathbf{v}+\left\{d_{\mathbf{x}} \mathbf{v}\right\}^{T}\right|^{2}\right)=-4 \pi G M \\
\operatorname{curl}_{\mathbf{x}}\left(\operatorname{curl}_{\mathbf{x}} \mathbf{v}\right)=0
\end{array}\right.
$$


where $\mathbf{D}, \mathbf{B}, \mathbf{E}, \mathbf{H}$ are given by (6.8), so that:

$$
\left\{\begin{array}{l}
\mathbf{B}=\operatorname{curl}_{\mathbf{x}} \mathbf{A} \\
\mathbf{D}=-\nabla_{\mathbf{x}} \Psi-\frac{\partial \mathbf{A}}{\partial x^{0}}+\mathbf{v} \times \operatorname{curl}_{\mathbf{x}} \mathbf{A} \\
\mathbf{E}=-\nabla_{\mathbf{x}} \Psi-\frac{\partial \mathbf{A}}{\partial x^{0}}=\mathbf{D}-\mathbf{v} \times \mathbf{B} \\
\mathbf{H}=\operatorname{curl}_{\mathbf{x}} \mathbf{A}+\mathbf{v} \times\left(-\nabla_{\mathbf{x}} \Psi-\frac{\partial \mathbf{A}}{\partial x^{0}}+\mathbf{v} \times \operatorname{curl}_{\mathbf{x}} \mathbf{A}\right)=\mathbf{B}+\mathbf{v} \times \mathbf{D}
\end{array}\right.
$$

\section{Polarization and magnetization}

It is well known from Tensor Analysis that if $\left\{\Upsilon^{m n}\right\}_{m, n=0,1,2,3}$ is an antisymmetric two-times contravariant tensor and if $\left\{K^{m n}\right\}_{m, n=0,1,2,3}$ is a contravariant pseudo-metric, then the four-component field $\left(\gamma^{0}, \gamma^{1}, \gamma^{2}, \gamma^{3}\right)$ defined by

$$
\begin{aligned}
\gamma^{m}:=\sum_{j=0}^{3} \frac{\partial S^{m j}}{\partial x^{j}}+\sum_{j=0}^{3} S^{m j} \frac{\frac{\partial}{\partial x^{j}}\left(\left|\operatorname{det}\left(\left\{K^{p q}\right\}_{p, q=0,1,2,3}\right)\right|^{-\frac{1}{2}}\right)}{\left|\operatorname{det}\left(\left\{K^{p q}\right\}_{p, q=0,1,2,3}\right)\right|^{-\frac{1}{2}}} \\
=\sum_{j=0}^{3} \frac{1}{\left|\operatorname{det}\left(\left\{K^{p q}\right\}_{p, q=0,1,2,3}\right)\right|^{-\frac{1}{2}}} \frac{\partial}{\partial x^{j}}\left\{\left|\operatorname{det}\left(\left\{K^{p q}\right\}_{p, q=0,1,2,3}\right)\right|^{-\frac{1}{2}} S^{m j}\right\} \quad \forall m=0,1,2,3,
\end{aligned}
$$

is a valid contravariant four-vector.

Lemma 9.1. Consider an arbitrary moving point with four dimensional trajectory $z(s)=\left(z^{0}(s), z^{1}(s), z^{2}(s), z^{3}(s)\right):[a, b] \rightarrow \mathbb{R}^{4}$. Moreover, assume that the infinite trajectory of the motion $z(s)$ is considered for all instances of time from $-\infty$ to $+\infty$ so that

$$
\lim _{s \rightarrow a^{-}} \sum_{j=0}^{3}\left(\left(z^{j}(s)\right)^{2}\right)=\lim _{s \rightarrow b^{+}} \sum_{j=0}^{3}\left(\left(z^{j}(s)\right)^{2}\right)=+\infty
$$

Next consider a point charge $\sigma$ with four dimensional trajectory $\chi(s)=\left(\chi^{0}(s), \chi^{1}(s), \chi^{2}(s), \chi^{3}(s)\right)$ : $[a, b] \rightarrow \mathbb{R}^{4}$, parameterized by the same proper parameter $s \in[a, b]$. Moreover, assume that the infinite trajectory of the motion $\chi(s)$ is considered for all instances of time from $-\infty$ to $+\infty$ so that for every $\tau \in[0,1]$ we have

$$
\lim _{s \rightarrow a^{-}} \sum_{j=0}^{3}\left(\left(\tau \chi^{j}(s)+(1-\tau) z^{j}(s)\right)^{2}\right)=\lim _{s \rightarrow b^{+}} \sum_{j=0}^{3}\left(\left(\tau \chi^{j}(s)+(1-\tau) z^{j}(s)\right)^{2}\right)=+\infty .
$$

If we define the antisymmetric two-times contravariant tensor $\left\{\Upsilon_{\sigma}^{m n}\right\}_{m, n=0,1,2,3}$ by:

$$
\begin{gathered}
\Upsilon_{\sigma}^{m n}\left(x^{0}, x^{1}, x^{2}, x^{3}\right):=\left|\operatorname{det}\left(\left\{K^{p q}\left(x^{0}, x^{1}, x^{2}, x^{3}\right)\right\}_{p, q=0,1,2,3}\right)\right|^{\frac{1}{2}} \int_{a}^{b} \int_{0}^{1}(\sigma\{ \\
\left(\chi^{m}(s)-z^{m}(s)\right)\left(\tau \frac{d \chi^{n}}{d s}(s)+(1-\tau) \frac{d z^{n}}{d s}(s)\right)-\left(\chi^{n}(s)-z^{n}(s)\right)\left(\tau \frac{d \chi^{m}}{d s}(s)+(1-\tau) \frac{d z^{m}}{d s}(s)\right) \\
\left\{\delta\left(x^{0}-\left(\tau \chi^{0}(s)+(1-\tau) z^{0}(s)\right), \ldots, x^{3}-\left(\tau \chi^{3}(s)+(1-\tau) z^{3}(s)\right)\right)\right) d \tau d s .
\end{gathered}
$$


Then, we have

$$
\begin{aligned}
& \sum_{n=0}^{3} \frac{1}{\left|\operatorname{det}\left(\left\{K^{p q}\right\}_{p, q=0,1,2,3}\right)\right|^{-\frac{1}{2}}} \frac{\partial}{\partial x^{n}}\left\{\left|\operatorname{det}\left(\left\{K^{p q}\right\}_{(p, q)_{0}^{3}}\right)\right|^{-\frac{1}{2}} \Upsilon_{\sigma}^{m n}\right\}= \\
& \left|\operatorname{det}\left(\left\{K^{p q}\right\}_{p, q=0,1,2,3}\right)\right|^{\frac{1}{2}} \int_{a}^{b} \sigma \frac{d \chi^{m}}{d s}(s) \delta\left(x^{0}-\chi^{0}(s), \ldots, x^{3}-\chi^{3}(s)\right) d s \\
& -\left|\operatorname{det}\left(\left\{K^{p q}\right\}_{p, q=0,1,2,3}\right)\right|^{\frac{1}{2}} \int_{a}^{b} \sigma \frac{d z^{m}}{d s}(s) \delta\left(x^{0}-z^{0}(s), \ldots, x^{3}-z^{3}(s)\right) d s
\end{aligned}
$$

in the sense of distributions.

Definition 9.1. Consider an arbitrary moving point with four dimensional trajectory $z(s)=$ $\left(z^{0}(s), z^{1}(s), z^{2}(s), z^{3}(s)\right):[a, b] \rightarrow \mathbb{R}^{4}$. Moreover, assume that the infinite trajectory of the motion $z(s)$ is considered for all instances of time from $-\infty$ to $+\infty$ so that

$$
\lim _{s \rightarrow a^{-}} \sum_{j=0}^{3}\left(\left(z^{j}(s)\right)^{2}\right)=\lim _{s \rightarrow b^{+}} \sum_{j=0}^{3}\left(\left(z^{j}(s)\right)^{2}\right)=+\infty .
$$

Next consider a totally neutral system of $N$ point charges $\sigma_{1}, \sigma_{2}, \ldots, \sigma_{N}$ satisfying

$$
\sum_{k=1}^{N} \sigma_{k}=0
$$

with four dimensional trajectory of the $k$-th particle $\chi_{k}(s)=\left(\chi_{k}^{0}(s), \chi_{k}^{1}(s), \chi_{k}^{2}(s), \chi_{k}^{3}(s)\right):[a, b] \rightarrow \mathbb{R}^{4}$ $\forall k=1,2, \ldots N$, parameterized by the same proper parameter $s \in[a, b]$. Moreover, assume that the infinite trajectory of the motion $\chi_{k}(s)$ is considered for all instances of time from $-\infty$ to $+\infty$ so that for every $\tau \in[0,1]$ we have

$$
\begin{array}{r}
\lim _{s \rightarrow a^{-}} \sum_{j=0}^{3}\left(\left(\tau \chi_{k}^{j}(s)+(1-\tau) z^{j}(s)\right)^{2}\right)=\lim _{s \rightarrow b^{+}} \sum_{j=0}^{3}\left(\left(\tau \chi_{k}^{j}(s)+(1-\tau) z^{j}(s)\right)^{2}\right)=+\infty \\
\forall k=1,2, \ldots, N .
\end{array}
$$

Then define the antisymmetric two-times contravariant tensor $\left\{\Upsilon^{m n}\right\}_{m, n=0,1,2,3}$ by:

$$
\begin{aligned}
& \Upsilon^{m n}\left(x^{0}, x^{1}, x^{2}, x^{3}\right):=\sum_{k=1}^{N}\left|\operatorname{det}\left(\left\{K^{p q}\left(x^{0}, x^{1}, x^{2}, x^{3}\right)\right\}_{p, q=0,1,2,3}\right)\right|^{\frac{1}{2}} \int_{a}^{b} \int_{0}^{1}\left(\sigma_{k}\{\right. \\
&\left(\chi_{k}^{m}(s)-z^{m}(s)\right)\left(\tau \frac{d \chi_{k}^{n}}{d s}(s)+(1-\tau) \frac{d z^{n}}{d s}(s)\right)-\left(\chi_{k}^{n}(s)-z^{n}(s)\right)\left(\tau \frac{d \chi_{k}^{m}}{d s}(s)+(1-\tau) \frac{d z^{m}}{d s}(s)\right) \\
&\} \delta\left(x^{0}-\left(\tau \chi_{k}^{0}(s)+(1-\tau) z^{0}(s)\right), \ldots, x^{3}-\left(\tau \chi_{k}^{3}(s)+(1-\tau) z^{3}(s)\right)\right)\right) d \tau d s,
\end{aligned}
$$

so that

$$
\Upsilon^{m n}\left(x^{0}, x^{1}, x^{2}, x^{3}\right):=\sum_{k=1}^{N} \Upsilon_{\sigma_{k}}^{m n}\left(x^{0}, x^{1}, x^{2}, x^{3}\right)
$$


Then by Lemma 9.1 we have

$$
\begin{aligned}
& \sum_{n=0}^{3} \frac{1}{\left|\operatorname{det}\left(\left\{K^{p q}\right\}_{p, q=0,1,2,3}\right)\right|^{-\frac{1}{2}} \frac{\partial}{\partial x^{n}}\left\{\left|\operatorname{det}\left(\left\{K^{p q}\right\}_{p, q=0,1,2,3}\right)\right|^{-\frac{1}{2}} \Upsilon^{m n}\right\}=} \\
& \quad \sum_{k=1}^{N} \sum_{n=0}^{3} \frac{1}{\left|\operatorname{det}\left(\left\{K^{p q}\right\}_{p, q=0,1,2,3}\right)\right|^{-\frac{1}{2}}} \frac{\partial}{\partial x^{n}}\left\{\left|\operatorname{det}\left(\left\{K^{p q}\right\}_{p, q=0,1,2,3}\right)\right|^{-\frac{1}{2}} \Upsilon_{\sigma}^{m n}\right\}= \\
& \quad \sum_{k=1}^{N}\left|\operatorname{det}\left(\left\{K^{p q}\right\}_{p, q=0,1,2,3}\right)\right|^{\frac{1}{2}} \int_{a}^{b} \sigma_{k} \frac{d \chi_{k}^{m}}{d s}(s) \delta\left(x^{0}-\chi_{k}^{0}(s), \ldots, x^{3}-\chi_{k}^{3}(s)\right) d s \\
& -\left|\operatorname{det}\left(\left\{K^{p q}\right\}_{p, q=0,1,2,3}\right)\right|^{\frac{1}{2}} \int_{a}^{b}\left(\sum_{k=1}^{N} \sigma_{k}\right) \frac{d z^{m}}{d s}(s) \delta\left(x^{0}-z^{0}(s), \ldots, x^{3}-z^{3}(s)\right) d s .
\end{aligned}
$$

Therefore, by inserting (9.7) into (9.11) we deduce

$$
\begin{aligned}
& \sum_{n=0}^{3} \frac{1}{\left|\operatorname{det}\left(\left\{K^{p q}\right\}_{p, q=0,1,2,3}\right)\right|^{-\frac{1}{2}} \frac{\partial}{\partial x^{n}}\left\{\left|\operatorname{det}\left(\left\{K^{p q}\right\}_{p, q=0,1,2,3}\right)\right|^{-\frac{1}{2}} \Upsilon^{m n}\right\}=} \\
& \\
& \sum_{k=1}^{N}\left|\operatorname{det}\left(\left\{K^{p q}\right\}_{p, q=0,1,2,3}\right)\right|^{\frac{1}{2}} \int_{a}^{b} \sigma_{k} \frac{d \chi_{k}^{m}}{d s}(s) \delta\left(x^{0}-\chi_{k}^{0}(s), \ldots, x^{3}-\chi_{k}^{3}(s)\right) d s=j^{m},
\end{aligned}
$$

in the sense of distributions, where the contravariant four-vector of the charge current $\left(j^{0}, j^{1}, j^{2}, j^{3}\right)$ is given as in (3.16).

Definition 9.2. Consider a totally neutral system of $N$ point charges $\sigma_{1}, \sigma_{2}, \ldots, \sigma_{N}$ satisfying

$$
\sum_{k=1}^{N} \sigma_{k}=0
$$

with four dimensional trajectory of the $k$-th particle $\chi_{k}(s)=\left(\chi_{k}^{0}(s), \chi_{k}^{1}(s), \chi_{k}^{2}(s), \chi_{k}^{3}(s)\right):[a, b] \rightarrow \mathbb{R}^{4}$ $\forall k=1,2, \ldots N$, parameterized by the same proper parameter $s \in[a, b]$. We define the multipole momentum $\mathcal{D}(s)=\left(\mathcal{D}^{0}(s), \mathcal{D}^{1}(s), \mathcal{D}^{2}(s), \mathcal{D}^{3}(s)\right):[a, b] \rightarrow \mathbb{R}^{4}$ of this system by:

$$
\mathcal{D}^{j}(s):=\sum_{k=1}^{N} \sigma_{k} \chi_{k}^{j}(s)=\sum_{k=1}^{N} \sigma_{k}\left(\chi_{k}^{j}(s)-z^{j}(s)\right) \quad \forall j=0,1,2,3 \quad \forall s \in[a, b],
$$

where $z(s)=\left(z^{0}(s), z^{1}(s), z^{2}(s), z^{3}(s)\right):[a, b] \rightarrow \mathbb{R}^{4}$ is a four-dimensional trajectory of an arbitrary moving point.

Definition 9.3. Consider an arbitrary moving point with four dimensional trajectory $z(s)=$ $\left(z^{0}(s), z^{1}(s), z^{2}(s), z^{3}(s)\right):[a, b] \rightarrow \mathbb{R}^{4}$. Moreover, consider a totally neutral system of $N$ point charges $\sigma_{1}, \sigma_{2}, \ldots, \sigma_{N}$ (possibly with infinitesimally large absolute values) satisfying

$$
\sum_{k=1}^{N} \sigma_{k}=0
$$


with four dimensional trajectory of the $k$-th particle,

$$
\chi_{k}(s):=\left(z^{0}(s)+l_{k}^{0}(s), z^{1}(s)+l_{k}^{1}(s), z^{2}(s)+l_{k}^{2}(s), z^{3}(s)+l_{k}^{3}(s)\right):[a, b] \rightarrow \mathbb{R}^{4} \quad \forall k=1,2, \ldots N,
$$

parameterized by the same proper parameter $s \in[a, b]$, and such that $\chi_{k}(s)$ is infinitesimally close to $z(s)$ for every $s \in[a, b]$ and every $k=1,2, \ldots, N$, so that $l_{k}^{j}(s)$ is infinitesimally small for all $j=0,1,2,3$ and all $k=1,2, \ldots N$. In particular this system of charges could be a dipole or the limiting cases of the totally neutral polarized molecule. Then we define the infinitesimal multipole momentum $\mathcal{D}(z(s))=\left(\mathcal{D}^{0}(z(s)), \mathcal{D}^{1}(z(s)), \mathcal{D}^{2}(z(s)), \mathcal{D}^{3}(z(s))\right):[a, b] \rightarrow \mathbb{R}^{4}$ of this system, with respect to the center $z(s)$, as follows:

$$
\mathcal{D}^{j}(z(s)):=\sum_{k=1}^{N} \sigma_{k}\left(\left\{z^{j}+l_{k}^{j}\right\}(s)-z^{j}(s)\right)=\sum_{k=1}^{N} \sigma_{k} l_{k}^{j}(s) \quad \forall j=0,1,2,3 \quad \forall s \in[a, b] .
$$

Although in this definition $l_{k}^{j}(s)$ is assumed to be infinitesimally small and $\sigma_{k}$ is allowed to be infinitesimally large, the quantity $\mathcal{D}^{j}(s)$ in (9.16) is always assumed to be finite.

Remark 9.1. Obviously, the infinitesimal multipole momentum, defined by (9.16) is a valid contravariant four-vector at the point $z(s)$. Indeed since, $l_{k}^{j}(s)$ is infinitesimally small, then under the change of the coordinate system given by a smooth non-degenerate invertible transformation from $\mathbb{R}^{4}$ onto $\mathbb{R}^{4}$, having the form

$$
\left\{\begin{array}{l}
x^{0}=f^{(0)}\left(x^{0}, x^{1}, x^{2}, x^{3}\right), \\
x^{\prime 1}=f^{(1)}\left(x^{0}, x^{1}, x^{2}, x^{3}\right), \\
x^{\prime 2}=f^{(2)}\left(x^{0}, x^{1}, x^{2}, x^{3}\right), \\
x^{\prime 3}=f^{(3)}\left(x^{0}, x^{1}, x^{2}, x^{3}\right),
\end{array}\right.
$$

we obviously have

$$
\begin{aligned}
\mathcal{D}^{\prime j}\left(z^{\prime}\right)=\sum_{k=1}^{N} \sigma_{k}\left(\left\{z^{\prime j}+l^{\prime j} k\right\}-z^{\prime j}\right)=\sum_{k=1}^{N} \sigma_{k}\left(f^{(j)}\left(z^{0}+l_{k}^{0}, \ldots, z^{3}+l_{k}^{3}\right)-f^{(j)}(z)\right) \\
\rightarrow \sum_{k=1}^{N} \sigma_{k}\left(\sum_{n=0}^{3} \frac{\partial f^{(j)}}{\partial x^{n}}(z) l_{k}^{n}\right)=\sum_{n=0}^{3} \frac{\partial f^{(j)}}{\partial x^{n}}(z) \mathcal{D}^{n}(z) \quad \forall j=0,1,2,3 .
\end{aligned}
$$

Definition 9.4. Consider an arbitrary moving point with four dimensional trajectory $z(s)=$ $\left(z^{0}(s), z^{1}(s), z^{2}(s), z^{3}(s)\right):[a, b] \rightarrow \mathbb{R}^{4}$. Moreover, assume that the infinite trajectory of the motion $z(s)$ is considered for all instances of time from $-\infty$ to $+\infty$ so that

$$
\lim _{s \rightarrow a^{-}} \sum_{j=0}^{3}\left(\left(z^{j}(s)\right)^{2}\right)=\lim _{s \rightarrow b^{+}} \sum_{j=0}^{3}\left(\left(z^{j}(s)\right)^{2}\right)=+\infty .
$$

Next, given an infinitesimal multipole at the point $z(s)$ with the infinitesimal multipole momentum $\mathcal{D}(z(s))=\left(\mathcal{D}^{0}(z(s)), \mathcal{D}^{1}(z(s)), \mathcal{D}^{2}(z(s)), \mathcal{D}^{3}(z(s))\right):[a, b] \rightarrow \mathbb{R}^{4}$, define the two-times contravariant antisymmetric tensor field of polarization-magnetization of this infinitesimal multipole 
$\left\{P^{m n}\right\}_{n, m=0,1,2,3}$ by:

$$
\begin{aligned}
& P^{m n}\left(x^{0}, x^{1}, x^{2}, x^{3}\right):=\int_{a}^{b}\left\{\mathcal{D}^{m}(z(s)) \frac{d z^{n}}{d s}(s)-\mathcal{D}^{n}(z(s)) \frac{d z^{m}}{d s}(s)\right. \\
& \qquad\left|\operatorname{det}\left(\left\{K^{p q}\left(z_{k}(s)\right)\right\}_{p, q=0,1,2,3}\right)\right|^{\frac{1}{2}} \delta\left(x^{0}-z^{0}(s), \ldots, x^{3}-z^{3}(s)\right) d s \\
& \forall m, n=0,1,2,3 \quad \forall\left(x^{0}, x^{1}, x^{2}, x^{3}\right) \in \mathbb{R}^{4} .
\end{aligned}
$$

Remark 9.2. Consider an arbitrary moving point with four dimensional trajectory $z(s)=\left(z^{0}(s), z^{1}(s), z^{2}(s), z^{3}(s)\right):[a, b] \rightarrow \mathbb{R}^{4}$. Moreover, consider a totally neutral system of $N$ point charges $\sigma_{1}, \sigma_{2}, \ldots, \sigma_{N}$ (possibly with infinitesimally large absolute values) satisfying

$$
\sum_{k=1}^{N} \sigma_{k}=0
$$

with four dimensional trajectory of the $k$-th particle,

$$
\chi_{k}(s):=\left(z^{0}(s)+l_{k}^{0}(s), z^{1}(s)+l_{k}^{1}(s), z^{2}(s)+l_{k}^{2}(s), z^{3}(s)+l_{k}^{3}(s)\right):[a, b] \rightarrow \mathbb{R}^{4} \quad \forall k=1,2, \ldots N
$$

parameterized by the same proper parameter $s \in[a, b]$, and such that $\chi_{k}(s)$ is infinitesimally close to $z(s)$ for every $s \in[a, b]$ and every $k=1,2, \ldots, N$, so that $l_{k}^{j}(s)$ is infinitesimally small for all $j=0,1,2,3$ and all $k=1,2, \ldots N$. Then, since $l_{k}^{j}(s)$ is infinitesimally small, by (9.9), (9.16) and (9.20) we deduce

$$
\Upsilon^{m n}\left(x^{0}, x^{1}, x^{2}, x^{3}\right) \rightarrow P^{m n}\left(x^{0}, x^{1}, x^{2}, x^{3}\right) \quad \forall m, n=0,1,2,3 \quad \forall\left(x^{0}, x^{1}, x^{2}, x^{3}\right) \in \mathbb{R}^{4}
$$

Therefore, by (9.22) we can rewrite (9.12) as:

$$
\sum_{n=0}^{3} \frac{1}{\left|\operatorname{det}\left(\left\{K^{p q}\right\}_{p, q=0,1,2,3}\right)\right|^{-\frac{1}{2}}} \frac{\partial}{\partial x^{n}}\left\{\left|\operatorname{det}\left(\left\{K^{p q}\right\}_{p, q=0,1,2,3}\right)\right|^{-\frac{1}{2}} P^{m n}\right\}=j^{m}
$$

where $\left(j^{0}, j^{1}, j^{2}, j^{3}\right)$ is the contravariant four-vector of the charge current, created by the infinitesimal multipole.

Definition 9.5. Given a union of finite number of infinitesimal multipoles, we define the two-times contravariant antisymmetric tensor field of polarization-magnetization $\left\{P^{m n}\right\}_{n, m=0,1,2,3}$ of this union as a sum of polarizations of every infinitesimal multipole in the union. Then, by (9.23) we have

$$
\sum_{n=0}^{3} \frac{1}{\left|\operatorname{det}\left(\left\{K^{p q}\right\}_{p, q=0,1,2,3}\right)\right|^{-\frac{1}{2}}} \frac{\partial}{\partial x^{n}}\left\{\left|\operatorname{det}\left(\left\{K^{p q}\right\}_{p, q=0,1,2,3}\right)\right|^{-\frac{1}{2}} P^{m n}\right\}=j_{p}^{m}
$$

where $\left(j_{p}^{0}, j_{p}^{1}, j_{p}^{2}, j_{p}^{3}\right)$ is the contravariant four-vector of the charge current of polarization, created by the union of infinitesimal multipoles. 
Next, given a moving dielectric and para/dia-magnetic continuum medium, by (2.115) we have the following Maxwell Equations:

$$
\begin{aligned}
& \sum_{j=0}^{3} \frac{\partial}{\partial x^{j}}\left(\sum_{m=0}^{3} \sum_{n=0}^{3}\left|\operatorname{det}\left\{K^{p q}\right\}_{p, q=0,1,2,3}\right|^{-\frac{1}{2}} K^{k m} K^{j n}\left(\frac{\partial A_{n}}{\partial x^{m}}-\frac{\partial A_{m}}{\partial x^{n}}\right)\right) \\
&=-4 \pi\left|\operatorname{det}\left\{K^{p q}\right\}_{p, q=0,1,2,3}\right|^{-\frac{1}{2}}\left(j^{k}+j_{p}^{k}\right) \quad \forall k=0,1,2,3,
\end{aligned}
$$

where $\left(j_{p}^{0}, j_{p}^{1}, j_{p}^{2}, j_{p}^{3}\right)$ is the contravariant four-vector of the charge current of polarization-magnetization, created by the dielectric and para/dia-magnetic medium (this medium can be represented as the union of infinitesimal multipoles) and $\left(j^{0}, j^{1}, j^{2}, j^{3}\right)$ is the contravariant four-vector of the free (real) charge current. Therefore, by (9.24) and (9.25) we deduce the following Maxwell Equations in the dielectric medium:

$$
\begin{aligned}
\sum_{j=0}^{3} \frac{\partial}{\partial x^{j}}\left\{\left|\operatorname{det}\left\{K^{p q}\right\}_{p, q=0,1,2,3}\right|^{-\frac{1}{2}}\right. & \left.\left(4 \pi P^{k j}+\sum_{m=0}^{3} \sum_{n=0}^{3} K^{k m} K^{j n}\left(\frac{\partial A_{n}}{\partial x^{m}}-\frac{\partial A_{m}}{\partial x^{n}}\right)\right)\right\} \\
& =-4 \pi\left|\operatorname{det}\left\{K^{p q}\right\}_{p, q=0,1,2,3}\right|^{-\frac{1}{2}} j^{k} \quad \forall k=0,1,2,3 .
\end{aligned}
$$

Next in the case of the simplest isotropic dielectric and para/dia-magnetic continuum medium we have

$$
\begin{aligned}
& P^{m n}=\sum_{j=0}^{3} \sum_{j=0}^{3} \kappa K^{m j} K^{n k}\left(\frac{\partial A_{k}}{\partial x^{j}}-\frac{\partial A_{j}}{\partial x^{k}}\right) \\
&+\sum_{k=0}^{3} \sum_{j=0}^{3}(\gamma-\kappa)\left(\sum_{k=0}^{3} \sum_{d=0}^{3} K_{k d} u^{k} u^{d}\right)^{-1}\left(K^{m j} u^{n} u^{k}+u^{m} u^{j} K^{n k}\right)\left(\frac{\partial A_{k}}{\partial x^{j}}-\frac{\partial A_{j}}{\partial x^{k}}\right)= \\
& \sum_{j=0}^{3} \sum_{j=0}^{3} \kappa K^{m j} K^{n k}\left(\frac{\partial A_{k}}{\partial x^{j}}-\frac{\partial A_{j}}{\partial x^{k}}\right) \\
&+\sum_{k=0}^{3} \sum_{j=0}^{3}(\gamma-\kappa)\left(\sum_{k=0}^{3} \sum_{d=0}^{3} K_{k d} u^{k} u^{d}\right)^{-1}\left(u^{n} K^{m j}-u^{m} K^{n j}\right) u^{k}\left(\frac{\partial A_{k}}{\partial x^{j}}-\frac{\partial A_{j}}{\partial x^{k}}\right) \quad \forall m, n=0,1,2,3 .
\end{aligned}
$$

where $\gamma$ and $\kappa$ are scalar fields and $\left(u^{1}, u^{2}, u^{3}, u^{4}\right)$ is the contravariant four-vector field of velocities of the moving dielectric continuum medium. In other words,

$$
\begin{array}{r}
P^{k j}=\sum_{j=0}^{3} \sum_{j=0}^{3} \kappa K^{j m} K^{k n}\left(\frac{\partial A_{n}}{\partial x^{m}}-\frac{\partial A_{m}}{\partial x^{n}}\right)+\sum_{m=0}^{3} \sum_{n=0}^{3}(\gamma-\kappa)\left(K^{k m} \tilde{u}^{j} \tilde{u}^{n}+\tilde{u}^{k} \tilde{u}^{m} K^{j n}\right)\left(\frac{\partial A_{n}}{\partial x^{m}}-\frac{\partial A_{m}}{\partial x^{n}}\right) \\
=\sum_{j=0}^{3} \sum_{j=0}^{3} \kappa K^{j m} K^{k n}\left(\frac{\partial A_{n}}{\partial x^{m}}-\frac{\partial A_{m}}{\partial x^{n}}\right)+\sum_{m=0}^{3} \sum_{n=0}^{3}(\gamma-\kappa)\left(\tilde{u}^{j} K^{k m}-\tilde{u}^{k} K^{j m}\right) \tilde{u}^{n}\left(\frac{\partial A_{n}}{\partial x^{m}}-\frac{\partial A_{m}}{\partial x^{n}}\right) \\
\forall k, j=0,1,2,3,
\end{array}
$$

where we denote

$$
\tilde{u}_{j}:=\left(\sum_{k=0}^{3} \sum_{d=0}^{3} K_{k d} u^{k} u^{d}\right)^{-\frac{1}{2}} u_{j} \quad \forall j=0,1,2,3
$$


Thus, inserting (9.28) into (9.26) implies

$$
\begin{array}{r}
\sum_{j=0}^{3} \frac{\partial}{\partial x^{j}}\left\{\sum_{m=0}^{3} \sum_{n=0}^{3}\left|\operatorname{det}\left\{K^{p q}\right\}_{p, q=0,1,2,3}\right|^{-\frac{1}{2}}\right. \\
\left.\left.(1+4 \pi \kappa)\left(K^{k m}+\frac{4 \pi(\gamma-\kappa)}{\sqrt{1+4 \pi \kappa}} \tilde{u}^{k} \tilde{u}^{m}\right)\left(K^{j n}+\frac{4 \pi(\gamma-\kappa)}{\sqrt{1+4 \pi \kappa}} \tilde{u}^{j} \tilde{u}^{n}\right)\right)\left(\frac{\partial A_{n}}{\partial x^{m}}-\frac{\partial A_{m}}{\partial x^{n}}\right)\right\} \\
=-4 \pi\left|\operatorname{det}\left\{K^{p q}\right\}_{p, q=0,1,2,3}\right|^{-\frac{1}{2}} j^{k} \quad \forall k=0,1,2,3 .
\end{array}
$$

(Note here that the normalized quantity $\left(\tilde{u}_{0}, \tilde{u}_{1}, \tilde{u}_{2}, \tilde{u}_{3}\right)$ is the same as the four-speed on the usual Theory of Relativity).

Finally, we consider the generally covariant Ohm's Law in a moving conducting medium of the form

$$
j^{m}-\left(\sum_{r=0}^{3} \sum_{k=0}^{3} K_{r k} \tilde{u}^{k} j^{r}\right) \tilde{u}^{m}=\sum_{r=0}^{3} \sum_{k=0}^{3} \varepsilon K^{m r} F_{r k} \tilde{u}^{k},
$$

where $\varepsilon$ is a certain scalar field and $\left\{F_{m n}\right\}_{n, m=0,1,2,3}$ is defined by

$$
F_{m j}:=\frac{\partial A_{j}}{\partial x^{m}}-\frac{\partial A_{m}}{\partial x^{j}} \quad \forall m, j=0,1,2,3 .
$$

Moreover, we consider the generally covariant Ohm's Law, including the Hall effect, of the form

$$
j^{m}-\left(\sum_{r=0}^{3} \sum_{k=0}^{3} K_{r k} \tilde{u}^{k} j^{r}\right) \tilde{u}^{m}=\sum_{r=0}^{3} \sum_{k=0}^{3} K^{m r} F_{r k}\left(\varepsilon \tilde{u}^{k}-\varsigma\left(j^{k}-\left(\sum_{r=0}^{3} \sum_{d=0}^{3} K_{r d} \tilde{u}^{d} j^{r}\right) \tilde{u}^{k}\right)\right),
$$

where $\varsigma$ is a certain scalar field.

\subsection{Polarization and magnetization in a cartesian coordinate system}

Consider the simplified model of the gravity, governed by the Lagrangian in (2.219), (2.220) and consider we deal with a cartesian coordinate system. Then, $\left\{K^{m n}\right\}_{n, m=0,1,2,3}$ is given by (4.29) and $\left\{K_{m n}\right\}_{n, m=0,1,2,3}$ is given by $(4.30)$, so that

$$
\begin{gathered}
\left\{\begin{array}{l}
K^{00}=1 \\
K^{j m}=-\delta_{j m}+v^{j} v^{m} \quad \forall 1 \leq j, m \leq 3 \\
K^{0 j}=K^{j 0}=v^{j} \quad \forall 1 \leq j \leq 3,
\end{array}\right. \\
\left\{\begin{array}{l}
K_{00}=1-|\mathbf{v}|^{2} \\
K_{j m}=-\delta_{j m} \quad \forall 1 \leq j, m \leq 3 \\
K_{0 j}=K_{j 0}=v^{j} \quad \forall 1 \leq j \leq 3,
\end{array}\right.
\end{gathered}
$$

where we denote

$$
\left(v^{0}, v^{1}, v^{2}, v^{3}\right):=(1, \mathbf{v}) \quad \text { with } \quad \mathbf{v}:=\left(v^{1}, v^{2}, v^{3}\right) \in \mathbb{R}^{3}
$$


Furthermore, consider $\left\{\Lambda^{m n}\right\}_{n, m=0,1,2,3}$ as

$$
\Lambda^{m n}=v^{m} v^{n}-K^{m n} \quad \forall m, n=0,1,2,3 .
$$

Then, by (9.33) $\left\{\Lambda^{m n}\right\}_{n, m=0,1,2,3}$ is given by

$$
\left\{\begin{array}{l}
\Lambda^{00}=0 \\
\Lambda^{j m}=\delta_{j m} \quad \forall 1 \leq j, m \leq 3 \\
\Lambda^{0 j}=\Lambda^{j 0}=0 \quad \forall 1 \leq j \leq 3 .
\end{array}\right.
$$

Next, let $\left\{F_{m n}\right\}_{n, m=0,1,2,3}$ be an anti-symmetric two-times covariant tensor, defined by

$$
F_{m j}:=\frac{\partial A_{j}}{\partial x^{m}}-\frac{\partial A_{m}}{\partial x^{j}} \quad \forall m, j=0,1,2,3 .
$$

Then, denoting

$$
\begin{gathered}
\quad\left(x^{0}, x^{1}, x^{2}, x^{3}\right)=\left(x^{0}, \mathbf{x}\right) \quad \text { where } \quad \mathbf{x}:=\left(x^{1}, x^{2}, x^{3}\right) \in \mathbb{R}^{3}, \\
\left(A_{0}, A_{1}, A_{2}, A_{3}\right)=(\Psi,-\mathbf{A}) \quad \text { where } \quad A_{0}=\Psi \text { and }\left(A_{1}, A_{2}, A_{3}\right)=-\mathbf{A}, \\
\left\{\begin{array}{l}
\mathbf{B}:=\left(B_{1}, B_{2}, B_{3}\right):=\operatorname{curl}_{\mathbf{x}} \mathbf{A}, \\
\mathbf{E}:=\left(E_{1}, E_{2}, E_{3}\right):=-\nabla_{\mathbf{x}} \Psi-\frac{\partial \mathbf{A}}{\partial x^{0}}, \\
\mathbf{D}^{(0)}:=\left(D_{1}^{(0)}, D_{2}^{(0)}, D_{3}^{(0)}\right):=\mathbf{E}+\mathbf{v} \times \mathbf{B}, \\
\mathbf{H}^{(0)}:=\left(H_{1}^{(0)}, H_{2}^{(0)}, H_{3}^{(0)}\right):=\mathbf{B}+\mathbf{v} \times \mathbf{D}^{(0)}=\mathbf{B}+\mathbf{v} \times(\mathbf{E}+\mathbf{v} \times \mathbf{B}),
\end{array}\right.
\end{gathered}
$$

and

$$
F^{m n}:=\sum_{k=0}^{3} \sum_{j=0}^{3} K^{m j} K^{n k} F_{j k}=\sum_{k=0}^{3} \sum_{j=0}^{3} K^{m j} K^{n k}\left(\frac{\partial A_{k}}{\partial x^{j}}-\frac{\partial A_{j}}{\partial x^{k}}\right) \quad \forall m, n=0,1,2,3,
$$

by Lemma 4.1 we have

and

$$
\left\{\begin{array}{l}
F_{00}=0 \\
F_{0 j}=-F_{j 0}=E_{j} \quad \forall j=1,2,3 \\
F_{j j}=0 \quad \forall j=1,2,3 \\
F_{12}=-F_{21}=-B_{3} \\
F_{13}=-F_{31}=B_{2} \\
F_{23}=-F_{32}=-B_{1},
\end{array}\right.
$$

$$
\left\{\begin{array}{l}
F^{00}=0 \\
F^{0 j}=-F^{j 0}=-D_{j}^{(0)} \quad \forall j=1,2,3 \\
F^{j j}=0 \quad \forall j=1,2,3 \\
F^{12}=-F^{21}=-H_{3}^{(0)} \\
F^{13}=-F^{31}=H_{2}^{(0)} \\
F^{23}=-F^{32}=-H_{1}^{(0)}
\end{array}\right.
$$


Next, given a moving dielectric and para/dia-magnetic medium, represented by a system of $N_{0}$ infinitesimal multipoles at the points

$$
\begin{aligned}
& z_{k}\left(x^{0}\right):=:\left(z_{k}^{0}\left(x^{0}\right), z_{k}^{1}\left(x^{0}\right), z_{k}^{2}\left(x^{0}\right), z_{k}^{3}\left(x^{0}\right)\right)=\left(x^{0}, \mathbf{z}_{k}\left(x^{0}\right)\right) \quad \text { where } \\
& \mathbf{z}_{k}\left(x^{0}\right):=\left(z_{k}^{1}\left(x^{0}\right), z_{k}^{2}\left(x^{0}\right), z_{k}^{3}\left(x^{0}\right)\right) \in \mathbb{R}^{3} \quad \forall k=1,2, \ldots, N_{0},
\end{aligned}
$$

parameterized by the first coordinate $z_{k}^{0}\left(z^{0}\right):=z^{0}$, with the infinitesimal multipole momentum $\mathcal{D}_{k}\left(z_{k}\left(z^{0}\right)\right):(-\infty,+\infty) \rightarrow \mathbb{R}^{4}$, given by

$$
\begin{aligned}
& \mathcal{D}_{k}\left(z_{k}\left(z^{0}\right)\right)=\left(\mathcal{D}_{k}^{0}\left(z_{k}\left(z^{0}\right)\right), \mathcal{D}_{k}^{1}\left(z_{k}\left(z^{0}\right)\right), \mathcal{D}_{k}^{2}\left(z_{k}\left(z^{0}\right)\right), \mathcal{D}_{k}^{3}\left(z_{k}\left(z^{0}\right)\right)\right)=\left(0, \mathbf{d}\left(z^{0}\right)\right) \\
& \text { where } \mathbf{d}\left(z^{0}\right):=\left(\mathcal{D}_{k}^{1}\left(z_{k}\left(z^{0}\right)\right), \mathcal{D}_{k}^{2}\left(z_{k}\left(z^{0}\right)\right), \mathcal{D}_{k}^{3}\left(z_{k}\left(z^{0}\right)\right)\right) \in \mathbb{R}^{3} \quad \forall k=1,2, \ldots, N_{0},
\end{aligned}
$$

where obviously we have $\mathcal{D}_{k}^{0}\left(z_{k}\left(z^{0}\right)\right)=0$, consider the two-times contravariant antisymmetric tensor $\left\{P^{m n}\right\}_{n, m=0,1,2,3}$, given by Definition 9.5 together with (9.20), so that we have

$$
\begin{aligned}
& P^{m n}\left(x^{0}, x^{1}, x^{2}, x^{3}\right)=\sum_{k=1}^{N_{0}} \int_{-\infty}^{+\infty}\left\{\mathcal{D}_{k}^{m}\left(z_{k}\left(z^{0}\right)\right) \frac{d z_{k}^{n}}{d z^{0}}\left(z^{0}\right)-\mathcal{D}_{k}^{n}\left(z_{k}\left(z^{0}\right)\right) \frac{d z_{k}^{m}}{d z^{0}}\left(z^{0}\right)\right. \\
& \delta\left(x^{0}-z_{k}^{0}\left(z^{0}\right), x^{1}-z_{k}^{1}\left(z^{0}\right), x^{2}-z_{k}^{2}\left(z^{0}\right), x^{3}-z_{k}^{3}\left(z^{0}\right)\right) d z^{0} \\
&=\sum_{k=1}^{N_{0}}\left\{\mathcal{D}_{k}^{m}\left(z_{k}\left(x^{0}\right)\right) \frac{d z_{k}^{n}}{d x^{0}}\left(x^{0}\right)-\mathcal{D}_{k}^{n}\left(z_{k}\left(x^{0}\right)\right) \frac{d z_{k}^{m}}{d x^{0}}\left(x^{0}\right)\right\} \delta\left(x^{1}-z_{k}^{1}\left(x^{0}\right), x^{2}-z_{k}^{2}\left(x^{0}\right), x^{3}-z_{k}^{3}\left(x^{0}\right)\right) \\
& \forall m, n=0,1,2,3 \quad \forall\left(x^{0}, x^{1}, x^{2}, x^{3}\right) \in \mathbb{R}^{4} .
\end{aligned}
$$

Thus, by (9.24) we have

$$
\sum_{n=0}^{3} \frac{\partial P^{m n}}{\partial x^{n}}=j_{p}^{m},
$$

In particular, by (9.47) we have

$$
\begin{array}{r}
P^{0 n}\left(x^{0}, x^{1}, x^{2}, x^{3}\right)=-\sum_{k=1}^{N_{0}} \mathcal{D}_{k}^{n}\left(z_{k}\left(x^{0}\right)\right) \delta\left(x^{1}-z_{k}^{1}\left(x^{0}\right), x^{2}-z_{k}^{2}\left(x^{0}\right), x^{3}-z_{k}^{3}\left(x^{0}\right)\right) \\
\forall n=0,1,2,3 \quad \forall\left(x^{0}, x^{1}, x^{2}, x^{3}\right) \in \mathbb{R}^{4} .
\end{array}
$$

Thus, considering $\mathbf{P}:=\left(P_{1}, P_{2}, P_{3}\right) \in \mathbb{R}^{3}$ and $\mathbf{M}:=\left(M_{1}, M_{2}, M_{3}\right) \in \mathbb{R}^{3}$, defined by

$$
\left\{\begin{array}{l}
P^{00}=0 \\
P^{0 j}=-P^{j 0}=-P_{j} \quad \forall j=1,2,3, \\
P^{j j}=0 \quad \forall j=1,2,3 \\
P^{12}=-P^{21}=-M_{3} \\
P^{13}=-P^{31}=M_{2} \\
P^{23}=-P^{32}=-M_{1}
\end{array}\right.
$$


by (9.49) and (9.47) we have

$$
\begin{array}{r}
\mathbf{P}\left(x^{0}, x^{1}, x^{2}, x^{3}\right)=\sum_{k=1}^{N_{0}} \mathbf{d}_{k}\left(z_{k}\left(x^{0}\right)\right) \delta\left(x^{1}-z_{k}^{1}\left(x^{0}\right), x^{2}-z_{k}^{2}\left(x^{0}\right), x^{3}-z_{k}^{3}\left(x^{0}\right)\right) \\
\forall m, n=0,1,2,3 \quad \forall\left(x^{0}, x^{1}, x^{2}, x^{3}\right) \in \mathbb{R}^{4},
\end{array}
$$

and

$$
\begin{array}{r}
\mathbf{M}\left(x^{0}, x^{1}, x^{2}, x^{3}\right)=\sum_{k=1}^{N_{0}} \frac{d \mathbf{z}_{k}}{d x^{0}}\left(x^{0}\right) \times \mathbf{d}_{k}\left(z_{k}\left(x^{0}\right)\right) \delta\left(x^{1}-z_{k}^{1}\left(x^{0}\right), x^{2}-z_{k}^{2}\left(x^{0}\right), x^{3}-z_{k}^{3}\left(x^{0}\right)\right) \\
\forall m, n=0,1,2,3 \quad \forall\left(x^{0}, x^{1}, x^{2}, x^{3}\right) \in \mathbb{R}^{4} .
\end{array}
$$

On the other hand by (9.50) and (9.48) we have

$$
\operatorname{div}_{\mathbf{x}} \mathbf{P}=-\sum_{n=1}^{3} \frac{\partial P^{0 n}}{\partial x^{n}}=-j_{p}^{0}=-\rho_{p}
$$

and

$$
\begin{aligned}
& \frac{\partial P_{1}}{\partial x^{0}}-\left(\frac{\partial M_{3}}{\partial x^{2}}-\frac{\partial M_{2}}{\partial x^{3}}\right)=\frac{\partial P^{10}}{\partial x^{0}}+\sum_{n=1}^{3} \frac{\partial P^{1 n}}{\partial x^{n}}=j_{p}^{1} \\
& \frac{\partial P_{2}}{\partial x^{0}}-\left(\frac{\partial M_{1}}{\partial x^{3}}-\frac{\partial M_{3}}{\partial x^{1}}\right)=\frac{\partial P^{20}}{\partial x^{0}}+\sum_{n=1}^{3} \frac{\partial P^{2 n}}{\partial x^{n}}=j_{p}^{2} \\
& \frac{\partial P_{3}}{\partial x^{0}}-\left(\frac{\partial M_{2}}{\partial x^{1}}-\frac{\partial M_{1}}{\partial x^{2}}\right)=\frac{\partial P^{30}}{\partial x^{0}}+\sum_{n=1}^{3} \frac{\partial P^{3 n}}{\partial x^{n}}=j_{p}^{3}
\end{aligned}
$$

so that

$$
\left\{\begin{array}{l}
\operatorname{div}_{\mathbf{x}} \mathbf{P}=-\rho_{p} \\
\frac{\partial \mathbf{P}}{\partial x^{0}}-\operatorname{curl}_{\mathbf{x}} \mathbf{M}=\mathbf{j}_{p} .
\end{array}\right.
$$

where as before, we denote $\left(x^{0}, x^{1}, x^{2}, x^{3}\right):=\left(x^{0}, \mathbf{x}\right)$ with $\mathbf{x}:=\left(x^{1}, x^{2}, x^{3}\right) \in \mathbb{R}^{3}$ and $\left(j_{p}^{0}, j_{p}^{1}, j_{p}^{2}, j_{p}^{3}\right):=$ $\left(\rho_{p}, \mathbf{j}_{p}\right)$ with $\mathbf{j}_{p}:=\left(j_{p}^{1}, j_{p}^{2}, j_{p}^{3}\right) \in \mathbb{R}^{3}$. However, by (9.42) and (9.33) we rewrite (9.25) as

$$
\sum_{j=0}^{3} \frac{\partial F^{k j}}{\partial x^{j}}=-4 \pi\left(j^{k}+j_{p}^{k}\right) \quad \forall k=0,1,2,3,
$$

where $F^{m n}$ is given by (9.44) and $\left(j^{0}, j^{1}, j^{2}, j^{3}\right):=(\rho, \mathbf{j})$ is the contravariant four vector of the free (real) charge current with $\mathbf{j}:=\left(j^{1}, j^{2}, j^{3}\right) \in \mathbb{R}^{3}$. Therefore, similarly to that we get in (9.57) we rewrite (9.58) as:

$$
\left\{\begin{array}{l}
\operatorname{div}_{\mathbf{x}} \mathbf{D}^{(0)}=4 \pi\left(\rho+\rho_{p}\right) \\
\operatorname{curl}_{\mathbf{x}} \mathbf{H}^{(0)}=4 \pi\left(\mathbf{j}+\mathbf{j}_{p}\right)+\frac{\partial \mathbf{D}^{(0)}}{\partial x^{0}}
\end{array}\right.
$$

where by (9.41) we have

$$
\left\{\begin{array}{l}
\mathbf{D}^{(0)}=\mathbf{E}+\mathbf{v} \times \mathbf{B}, \\
\mathbf{H}^{(0)}=\mathbf{B}+\mathbf{v} \times \mathbf{D}^{(0)}=\mathbf{B}+\mathbf{v} \times(\mathbf{E}+\mathbf{v} \times \mathbf{B}) .
\end{array}\right.
$$


Thus, by (9.57) we rewrite (9.59) as:

$$
\left\{\begin{array}{l}
\operatorname{div}_{\mathbf{x}}\left(\mathbf{D}^{(0)}+4 \pi \mathbf{P}\right)=4 \pi \rho \\
\operatorname{curl}_{\mathbf{x}}\left(\mathbf{H}^{(0)}+4 \pi \mathbf{M}\right)=4 \pi \mathbf{j}+\frac{\partial}{\partial x^{0}}\left(\mathbf{D}^{(0)}+4 \pi \mathbf{P}\right) .
\end{array}\right.
$$

Therefore, denoting:

$$
\left\{\begin{array}{l}
\mathbf{D}:=\mathbf{D}^{(0)}+4 \pi \mathbf{P}=\mathbf{E}+\mathbf{v} \times \mathbf{B}+4 \pi \mathbf{P} \\
\mathbf{H}:=\mathbf{H}^{(0)}+4 \pi \mathbf{M}=\mathbf{B}+\mathbf{v} \times(\mathbf{E}+\mathbf{v} \times \mathbf{B})+4 \pi \mathbf{M}
\end{array}\right.
$$

we rewrite (9.61) or equivalently (9.26) in the cartesian coordinate system as:

$$
\left\{\begin{array}{l}
\operatorname{div}_{\mathbf{x}} \mathbf{D}=4 \pi \rho \\
\operatorname{curl}_{\mathbf{x}} \mathbf{H}=4 \pi \mathbf{j}+\frac{\partial \mathbf{D}}{\partial x^{0}} \\
\operatorname{curl}_{\mathbf{x}} \mathbf{E}+\frac{\partial \mathbf{B}}{\partial x^{0}}=0 \\
\operatorname{div}_{\mathbf{x}} \mathbf{B}=0
\end{array}\right.
$$

where $\mathbf{D}$ is the electric displacement field and $\mathbf{H}$ is an auxiliary magnetic field in the dielectric medium, given by

$$
\left\{\begin{array}{l}
\mathbf{D}:=\mathbf{E}+\mathbf{v} \times \mathbf{B}+4 \pi \mathbf{P} \\
\mathbf{H}:=\mathbf{B}+\mathbf{v} \times(\mathbf{E}+\mathbf{v} \times \mathbf{B})+4 \pi \mathbf{M}
\end{array}\right.
$$

Next consider two-times contravariant tensor $\left\{G^{m n}\right\}_{m, n=0,1,2,3}$ defined by:

$$
\begin{array}{r}
G^{m n}:=\sum_{k=0}^{3} \sum_{j=0}^{3}\left(K^{m j} u^{n} u^{k}+u^{m} u^{j} K^{n k}\right) F_{j k}=\sum_{k=0}^{3} \sum_{j=0}^{3}\left(u^{n} K^{m j}-u^{m} K^{n j}\right) u^{k} F_{j k} \\
\forall m, n=0,1,2,3,
\end{array}
$$

where $\left(u^{1}, u^{2}, u^{3}, u^{4}\right)$ is the contravariant four-vector field of velocities of the moving dielectric continuum medium and we have

$$
\left(u^{0}, u^{1}, u^{2}, u^{3}\right):=(1, \mathbf{u}) \quad \text { with } \quad \mathbf{u}:=\left(u^{1}, u^{2}, u^{3}\right) \in \mathbb{R}^{3} .
$$

In particular, since the tensor $\left\{G^{m n}\right\}_{m, n=0,1,2,3}$ is antisymmetric, i.e. $G^{m n}=-G^{n m} \forall m, n=$ $0,1,2,3$, then we have

$$
\left\{\begin{array}{l}
G^{00}=0 \\
G^{m m}=0 \quad \forall m=1,2,3, \\
G^{0 m}=-G^{m 0} \\
G^{m n}=-G^{n m} \quad \forall m \neq n=1,2,3 .
\end{array}\right.
$$


Next by (9.36), since $\left\{F_{j k}\right\}_{j, k=0,1,2,3}$ is an antisymmetric matrix, we rewrite (9.65) as:

$$
\begin{gathered}
G^{m n}=\sum_{k=0}^{3} \sum_{j=0}^{3}\left(u^{n}\left(v^{m} v^{j}-\Lambda^{m j}\right)-u^{m}\left(v^{n} v^{j}-\Lambda^{n j}\right)\right) u^{k} F_{j k}=-\sum_{k=0}^{3} \sum_{j=0}^{3}\left(u^{n} \Lambda^{m j}-u^{m} \Lambda^{n j}\right) u^{k} F_{j k} \\
\quad+\sum_{k=0}^{3} \sum_{j=0}^{3}\left(u^{n} v^{m} v^{j}-u^{m} v^{n} v^{j}\right) u^{k} F_{j k}=-\sum_{k=0}^{3} \sum_{j=0}^{3}\left(u^{n} \Lambda^{m j}-u^{m} \Lambda^{n j}\right) u^{k} F_{j k} \\
\quad+\left(u^{n} v^{m}-u^{m} v^{n}\right)\left(\sum_{j=0}^{3} \sum_{k=0}^{3} v^{j} u^{k} F_{j k}\right)=-\sum_{k=0}^{3} \sum_{j=0}^{3}\left(u^{n} \Lambda^{m j}-u^{m} \Lambda^{n j}\right) u^{k} F_{j k} \\
+\sum_{k=0}^{3} \sum_{j=0}^{3}\left(u^{n}\left(v^{m}-u^{m}\right)-u^{m}\left(v^{n}-u^{n}\right)\right)\left(\sum_{j=0}^{3} \sum_{k=0}^{3}\left(v^{j}-u^{j}\right) u^{k} F_{j k}\right) \quad \forall m, n=0,1,2,3 .
\end{gathered}
$$

So,

$$
\begin{aligned}
G^{m n}= & -\sum_{k=0}^{3} \sum_{j=0}^{3}\left(u^{n} \Lambda^{m j}-u^{m} \Lambda^{n j}\right) u^{k} F_{j k} \\
& +\left(u^{n}\left(u^{m}-v^{m}\right)-u^{m}\left(u^{n}-v^{n}\right)\right)\left(\sum_{j=0}^{3} \sum_{k=0}^{3}\left(u^{j}-v^{j}\right) u^{k} F_{j k}\right) \quad \forall m, n=0,1,2,3 .
\end{aligned}
$$

Next consider two-times contravariant tensor $\left\{\tilde{G}^{m n}\right\}_{m, n=0,1,2,3}$ defined by:

$$
\begin{array}{r}
-\tilde{G}^{m n}:=\sum_{k=0}^{3} \sum_{j=0}^{3}\left(\Lambda^{m j} u^{n} u^{k}+u^{m} u^{j} \Lambda^{n k}\right) F_{j k}=\sum_{k=0}^{3} \sum_{j=0}^{3}\left(u^{n} \Lambda^{m j}-u^{m} \Lambda^{n j}\right) u^{k} F_{j k} \\
\forall m, n=0,1,2,3 .
\end{array}
$$

In particular, since the tensor $\left\{\tilde{G}^{m n}\right\}_{m, n=0,1,2,3}$ is antisymmetric, i.e. $\tilde{G}^{m n}=-\tilde{G}^{n m} \forall m, n=$ $0,1,2,3$, then we have

$$
\left\{\begin{array}{l}
\tilde{G}^{00}=0 \\
\tilde{G}^{m m}=0 \quad \forall m=1,2,3, \\
\tilde{G}^{0 m}=-\tilde{G}^{m 0} \\
\tilde{G}^{m n}=-\tilde{G}^{n m} \quad \forall m \neq n=1,2,3 .
\end{array}\right.
$$

On the other hand, by (9.69) and (9.70) together we deduce:

$$
G^{m n}=\tilde{G}^{m n}+\left(u^{n}\left(u^{m}-v^{m}\right)-u^{m}\left(u^{n}-v^{n}\right)\right)\left(\sum_{j=0}^{3} \sum_{k=0}^{3}\left(u^{j}-v^{j}\right) u^{k} F_{j k}\right) \quad \forall m, n=0,1,2,3 .
$$

In particular, in the case we have

$$
|\mathbf{u}-\mathbf{v}|^{2} \ll 1
$$


by (9.72), using (9.35) and (9.66), we rewrite (9.65) as

$$
\begin{gathered}
\left(\sum_{k=0}^{3} \sum_{d=0}^{3} K_{k d} u^{k} u^{d}\right)^{-1} G^{m n}=\sum_{k=0}^{3} \sum_{j=0}^{3}\left(\sum_{r=0}^{3} \sum_{d=0}^{3} K_{r d} u^{r} u^{d}\right)^{-1}\left(u^{n} K^{m j}-u^{m} K^{n j}\right) u^{k} F_{j k} \\
\approx \tilde{G}^{m n}=-\sum_{k=0}^{3} \sum_{j=0}^{3}\left(u^{n} \Lambda^{m j}-u^{m} \Lambda^{n j}\right) u^{k} F_{j k}
\end{gathered}
$$$$
\forall m, n=0,1,2,3 \text {. }
$$

Next we rewrite (9.70) as:

$$
\begin{gathered}
-\tilde{G}^{m n}=\sum_{k=0}^{3} \sum_{j=0}^{3}\left(u^{n} \Lambda^{m j}-u^{m} \Lambda^{n j}\right) u^{k} F_{j k}=\sum_{j=0}^{3}\left(u^{n} \Lambda^{m j}-u^{m} \Lambda^{n j}\right)\left(F_{j 0}+\sum_{k=1}^{3} u^{k} F_{j k}\right) \\
=\left(u^{n} \Lambda^{m 0}-u^{m} \Lambda^{n 0}\right)\left(F_{00}+\sum_{k=1}^{3} u^{k} F_{0 k}\right)+\sum_{j=1}^{3}\left(u^{n} \Lambda^{m j}-u^{m} \Lambda^{n j}\right)\left(F_{j 0}+\sum_{k=1}^{3} u^{k} F_{j k}\right) \\
=\left(u^{n} \Lambda^{m 0}-u^{m} \Lambda^{n 0}\right)\left(\sum_{k=1}^{3} u^{k} F_{0 k}\right)+\sum_{j=1}^{3}\left(u^{n} \Lambda^{m j}-u^{m} \Lambda^{n j}\right) F_{j 0} \\
+\sum_{j=1}^{3}\left(u^{n} \Lambda^{m j}-u^{m} \Lambda^{n j}\right)\left(\sum_{k=1}^{3} u^{k} F_{j k}\right) \quad \forall m, n=0,1,2,3 .
\end{gathered}
$$

So, by (9.37) and (9.75) we deduce

$$
\begin{aligned}
& \tilde{G}^{n 0}=-\tilde{G}^{0 n}= \\
& \left(u^{n} \Lambda^{00}-\Lambda^{n 0}\right)\left(\sum_{k=1}^{3} u^{k} F_{0 k}\right)+\sum_{j=1}^{3}\left(u^{n} \Lambda^{0 j}-\Lambda^{n j}\right) F_{j 0}+\sum_{j=1}^{3}\left(u^{n} \Lambda^{0 j}-\Lambda^{n j}\right)\left(\sum_{k=1}^{3} u^{k} F_{j k}\right)= \\
& -\sum_{j=1}^{3} \delta_{n j} F_{j 0}-\sum_{j=1}^{3} \delta_{n j}\left(\sum_{k=1}^{3} u^{k} F_{j k}\right)=-F_{n 0}-\sum_{k=1}^{3} u^{k} F_{n k}=\left(E^{n}-\sum_{k=1}^{3} u^{k} F_{n k}\right) \quad \forall n=1,2,3 .
\end{aligned}
$$

Therefore, by (9.43) and (9.76) we have

$$
\left\{\begin{array}{l}
\tilde{G}^{10}=-\tilde{G}^{01}=\left(E^{1}-u^{2} F_{12}-u^{3} F_{13}\right)=\left(E^{1}+\left(u^{2} B_{3}-u^{3} B_{2}\right)\right) \\
\tilde{G}^{20}=-\tilde{G}^{02}=\left(E^{2}-u^{3} F_{23}-u^{1} F_{21}\right)=\left(E^{2}+\left(u^{3} B_{1}-u^{1} B_{3}\right)\right) \\
\tilde{G}^{30}=-\tilde{G}^{03}=\left(E^{3}-u^{1} F_{31}-u^{2} F_{32}\right)=\left(E^{3}+\left(u^{1} B_{2}-u^{2} B_{1}\right)\right) .
\end{array}\right.
$$

On the other hand, by (9.37) and (9.75) we obtain

$$
\begin{gathered}
-\tilde{G}^{m n}=\sum_{j=1}^{3}\left(u^{n} \delta_{m j}-u^{m} \delta_{n j}\right) F_{j 0}+\sum_{j=1}^{3}\left(u^{n} \delta_{m j}-u^{m} \delta_{n j}\right)\left(\sum_{k=1}^{3} u^{k} F_{j k}\right) \\
=u^{n} F_{m 0}-u^{m} F_{n 0}+u^{n}\left(\sum_{k=1}^{3} u^{k} F_{m k}\right)-u^{m}\left(\sum_{k=1}^{3} u^{k} F_{n k}\right) \\
=-\left(u^{n}\left(E^{m}-\sum_{k=1}^{3} u^{k} F_{m k}\right)-u^{m}\left(E^{n}-\sum_{k=1}^{3} u^{k} F_{n k}\right)\right) \quad \forall m, n=1,2,3 .
\end{gathered}
$$


Thus, considering $\mathbf{G}:=\left(G_{1}, G_{2}, G_{3}\right) \in \mathbb{R}^{3}$ and $\mathbf{V}:=\left(V_{1}, V_{2}, V_{3}\right) \in \mathbb{R}^{3}$, defined by

$$
\left\{\begin{array}{l}
G^{00}=0 \\
G^{0 j}=-G^{j 0}=-G_{j} \quad \forall j=1,2,3, \\
G^{j j}=0 \quad \forall j=1,2,3 \\
G^{12}=-G^{21}=-V_{3} \\
G^{13}=-G^{31}=V_{2} \\
G^{23}=-G^{32}=-V_{1}
\end{array}\right.
$$

by (9.76), (9.78), (9.77) and (9.74), together with (9.72), we deduce:

$$
\left\{\begin{array}{l}
\left(\sum_{k=0}^{3} \sum_{d=0}^{3} K_{k d} u^{k} u^{d}\right)^{-1} \mathbf{G} \approx \mathbf{E}+\mathbf{u} \times \mathbf{B} \\
\left(\sum_{k=0}^{3} \sum_{d=0}^{3} K_{k d} u^{k} u^{d}\right)^{-1} \mathbf{V}=\left(\sum_{k=0}^{3} \sum_{d=0}^{3} K_{k d} u^{k} u^{d}\right)^{-1} \mathbf{u} \times \mathbf{G} \approx \mathbf{u} \times(\mathbf{E}+\mathbf{u} \times \mathbf{B}),
\end{array}\right.
$$

provided, we have (9.73). Next in the case of the simplest isotropic continuum medium we rewrite (9.27) as:

$$
P^{m n}=\sum_{j=0}^{3} \sum_{j=0}^{3} \kappa K^{m j} K^{n k} F_{j k}+(\gamma-\kappa)\left(\sum_{k=0}^{3} \sum_{d=0}^{3} K_{k d} u^{k} u^{d}\right)^{-1} G^{m n} \quad \forall m, n=0,1,2,3,
$$

where $\gamma$ ad $\kappa$ are scalar fields. Thus, by (9.50), (9.79) and (9.80), together with (9.81) and (9.44) we have

$$
\left\{\begin{array}{l}
\mathbf{P} \approx(\gamma-\kappa)(\mathbf{E}+\mathbf{u} \times \mathbf{B})+\kappa(\mathbf{E}+\mathbf{v} \times \mathbf{B}) \\
\mathbf{M} \approx(\gamma-\kappa) \mathbf{u} \times(\mathbf{E}+\mathbf{u} \times \mathbf{B})+\kappa(\mathbf{B}+\mathbf{v} \times(\mathbf{E}+\mathbf{v} \times \mathbf{B})) .
\end{array}\right.
$$

We rewrite (9.82) as:

$$
\left\{\begin{array}{l}
\mathbf{P} \approx \gamma(\mathbf{E}+\mathbf{u} \times \mathbf{B})-\kappa(\mathbf{u}-\mathbf{v}) \times \mathbf{B} \\
\mathbf{M} \approx \mathbf{u} \times(\gamma(\mathbf{E}+\mathbf{u} \times \mathbf{B}-\kappa(\mathbf{u}-\mathbf{v}) \times \mathbf{B}))+\kappa \mathbf{B}-\kappa(\mathbf{u}-\mathbf{v}) \times(\mathbf{E}+\mathbf{v} \times \mathbf{B}) .
\end{array}\right.
$$

Thus, by (9.83) we rewrite (9.64) as

$$
\begin{aligned}
\mathbf{D} \approx(1+4 \pi \gamma)\left(\mathbf{E}+\left(\frac{1}{1+4 \pi \gamma} \mathbf{v}+\frac{4 \pi \gamma}{1+4 \pi \gamma} \mathbf{u}-\frac{4 \pi \kappa}{1+4 \pi \gamma}(\mathbf{u}-\mathbf{v})\right) \times \mathbf{B}\right)= \\
(1+4 \pi \gamma)\left(\mathbf{E}+\left(\frac{1+4 \pi \kappa}{1+4 \pi \gamma} \mathbf{v}+\left(1-\frac{1+4 \pi \kappa}{1+4 \pi \gamma}\right) \mathbf{u}\right) \times \mathbf{B}\right)
\end{aligned}
$$


and

$$
\begin{gathered}
\mathbf{H} \approx(1+4 \pi \kappa) \mathbf{B}+(1+4 \pi \gamma)\left(\frac{1}{1+4 \pi \gamma} \mathbf{v}+\frac{4 \pi \gamma}{1+4 \pi \gamma} \mathbf{u}\right) \times \mathbf{E}+\mathbf{v} \times(\mathbf{v} \times \mathbf{B})+4 \pi \gamma \mathbf{u} \times(\mathbf{u} \times \mathbf{B}) \\
-4 \pi \kappa \mathbf{u} \times((\mathbf{u}-\mathbf{v}) \times \mathbf{B})-4 \pi \kappa(\mathbf{u}-\mathbf{v}) \times(\mathbf{E}+\mathbf{v} \times \mathbf{B})= \\
(1+4 \pi \kappa) \mathbf{B}+(1+4 \pi \gamma)\left(\frac{1}{1+4 \pi \gamma} \mathbf{v}+\frac{4 \pi \gamma}{1+4 \pi \gamma} \mathbf{u}-\frac{4 \pi \kappa}{1+4 \pi \gamma}(\mathbf{u}-\mathbf{v})\right) \times \mathbf{E}+\mathbf{v} \times(\mathbf{v} \times \mathbf{B}) \\
+4 \pi \gamma \mathbf{u} \times(\mathbf{u} \times \mathbf{B})-4 \pi \kappa \mathbf{u} \times((\mathbf{u}-\mathbf{v}) \times \mathbf{B})-4 \pi \kappa(\mathbf{u}-\mathbf{v}) \times(\mathbf{v} \times \mathbf{B})=(1+4 \pi \kappa) \mathbf{B} \\
+(1+4 \pi \gamma)\left(\frac{1+4 \pi \kappa}{1+4 \pi \gamma} \mathbf{v}+\left(1-\frac{1+4 \pi \kappa}{1+4 \pi \gamma}\right) \mathbf{u}\right) \times\left(\mathbf{E}+\left(\frac{1+4 \pi \kappa}{1+4 \pi \gamma} \mathbf{v}+\left(1-\frac{1+4 \pi \kappa}{1+4 \pi \gamma}\right) \mathbf{u}\right) \times \mathbf{B}\right) \\
-(1+4 \pi \gamma)\left(\frac{1+4 \pi \kappa}{1+4 \pi \gamma} \mathbf{v}+\left(1-\frac{1+4 \pi \kappa}{1+4 \pi \gamma}\right) \mathbf{u}\right) \times\left(\left(\frac{1+4 \pi \kappa}{1+4 \pi \gamma} \mathbf{v}+\left(1-\frac{1+4 \pi \kappa}{1+4 \pi \gamma}\right) \mathbf{u}\right) \times \mathbf{B}\right) \\
+\mathbf{v} \times(\mathbf{v} \times \mathbf{B})+4 \pi \gamma \mathbf{u} \times(\mathbf{u} \times \mathbf{B})-4 \pi \kappa \mathbf{u} \times((\mathbf{u}-\mathbf{v}) \times \mathbf{B})-4 \pi \kappa(\mathbf{u}-\mathbf{v}) \times(\mathbf{v} \times \mathbf{B}) .
\end{gathered}
$$

Thus, by (9.84) we rewrite (9.85) as:

$$
\begin{gathered}
\mathbf{H} \approx(1+4 \pi \kappa) \mathbf{B}+\left(\frac{1+4 \pi \kappa}{1+4 \pi \gamma} \mathbf{v}+\left(1-\frac{1+4 \pi \kappa}{1+4 \pi \gamma}\right) \mathbf{u}\right) \times \mathbf{D} \\
-(1+4 \pi \gamma)\left(\frac{1+4 \pi \kappa}{1+4 \pi \gamma} \mathbf{v}+\left(1-\frac{1+4 \pi \kappa}{1+4 \pi \gamma}\right) \mathbf{u}\right) \times\left(\left(\frac{1+4 \pi \kappa}{1+4 \pi \gamma} \mathbf{v}+\left(1-\frac{1+4 \pi \kappa}{1+4 \pi \gamma}\right) \mathbf{u}\right) \times \mathbf{B}\right) \\
+\mathbf{v} \times(\mathbf{v} \times \mathbf{B})+4 \pi \gamma \mathbf{u} \times(\mathbf{u} \times \mathbf{B})-4 \pi \kappa \mathbf{u} \times((\mathbf{u}-\mathbf{v}) \times \mathbf{B})-4 \pi \kappa(\mathbf{u}-\mathbf{v}) \times(\mathbf{v} \times \mathbf{B})= \\
(1+4 \pi \kappa) \mathbf{B}+\left(\frac{1+4 \pi \kappa}{1+4 \pi \gamma} \mathbf{v}+\left(1-\frac{1+4 \pi \kappa}{1+4 \pi \gamma}\right) \mathbf{u}\right) \times \mathbf{D} \\
+(1+4 \pi \kappa) \frac{(1+4 \pi \gamma)-(1+4 \pi \kappa)}{1+4 \pi \gamma}(\mathbf{u}-\mathbf{v}) \times((\mathbf{u}-\mathbf{v}) \times \mathbf{B})
\end{gathered}
$$

Therefore, denoting

$$
\left\{\begin{array}{l}
\gamma_{0}:=\frac{1}{1+4 \pi \gamma} \\
\kappa_{0}:=(1+4 \pi \kappa) \\
\hat{\mathbf{u}}:=\left(\gamma_{0} \kappa_{0} \mathbf{v}+\left(1-\gamma_{0} \kappa_{0}\right) \mathbf{u}\right)=\left(\frac{1+4 \pi \kappa}{1+4 \pi \gamma} \mathbf{v}+\left(1-\frac{1+4 \pi \kappa}{1+4 \pi \gamma}\right) \mathbf{u}\right)
\end{array}\right.
$$

and using (9.73) we rewrite (9.84) and (9.86) as:

$$
\left\{\begin{array}{l}
\mathbf{E}=\gamma_{0} \mathbf{D}-\hat{\mathbf{u}} \times \mathbf{B} \\
\mathbf{H}=\kappa_{0} \mathbf{B}+\hat{\mathbf{u}} \times \mathbf{D}+\kappa_{0}\left(1-\gamma_{0} \kappa_{0}\right)(\mathbf{u}-\mathbf{v}) \times((\mathbf{u}-\mathbf{v}) \times \mathbf{B}) .
\end{array}\right.
$$

In particular, if

$$
\left|\kappa_{0}\right|\left|1-\gamma_{0} \kappa_{0}\right||\mathbf{u}-\mathbf{v}|^{2} \ll 1,
$$

we have

$$
\left\{\begin{array}{l}
\mathbf{E}=\gamma_{0} \mathbf{D}-\hat{\mathbf{u}} \times \mathbf{B} \\
\mathbf{H}=\kappa_{0} \mathbf{B}+\hat{\mathbf{u}} \times \mathbf{D}
\end{array}\right.
$$


Thus, the case of (9.89), by (9.90) we rewrite (9.63), or equivalently we approximate (9.30) in the cartesian coordinate system as:

$$
\left\{\begin{array}{l}
\operatorname{div}_{\mathbf{x}} \mathbf{D}=4 \pi \rho \\
\operatorname{curl}_{\mathbf{x}} \mathbf{H}=4 \pi \mathbf{j}+\frac{\partial \mathbf{D}}{\partial x^{0}} \\
\operatorname{curl}_{\mathbf{x}} \mathbf{E}+\frac{\partial \mathbf{B}}{\partial x^{0}}=0 \\
\operatorname{div}_{\mathbf{x}} \mathbf{B}=0 \\
\mathbf{E}=\gamma_{0} \mathbf{D}-\left(\gamma_{0} \kappa_{0} \mathbf{v}+\left(1-\gamma_{0} \kappa_{0}\right) \mathbf{u}\right) \times \mathbf{B} \\
\mathbf{H}=\kappa_{0} \mathbf{B}+\left(\gamma_{0} \kappa_{0} \mathbf{v}+\left(1-\gamma_{0} \kappa_{0}\right) \mathbf{u}\right) \times \mathbf{D}
\end{array}\right.
$$

provided we have

$$
\left|\kappa_{0}\right|\left|1-\gamma_{0} \kappa_{0}\right||\mathbf{u}-\mathbf{v}|^{2} \ll 1
$$

Moreover, we can rewrite (9.91) as:

$$
\left\{\begin{array}{l}
\operatorname{div}_{\mathbf{x}} \mathbf{D}=4 \pi \rho \\
\operatorname{curl}_{\mathbf{x}}\left(\kappa_{0} \mathbf{B}\right)=4 \pi \mathbf{j}+\frac{\partial \mathbf{D}}{\partial x^{0}}-\operatorname{curl}_{\mathbf{x}}(\hat{\mathbf{u}} \times \mathbf{D}) \\
\operatorname{curl}_{\mathbf{x}}\left(\gamma_{0} \mathbf{D}\right)=-\left(\frac{\partial \mathbf{B}}{\partial x^{0}}-\operatorname{curl}_{\mathbf{x}}(\hat{\mathbf{u}} \times \mathbf{B})\right) \\
\operatorname{div}_{\mathbf{x}} \mathbf{B}=0 \\
\mathbf{E}=\gamma_{0} \mathbf{D}-\hat{\mathbf{u}} \times \mathbf{B} \\
\mathbf{H}=\kappa_{0} \mathbf{B}+\hat{\mathbf{u}} \times \mathbf{D} \\
\hat{\mathbf{u}}:=\left(\gamma_{0} \kappa_{0} \mathbf{v}+\left(1-\gamma_{0} \kappa_{0}\right) \mathbf{u}\right) .
\end{array}\right.
$$

We call $\gamma_{0}$ the dielectric permeability of the dielectric medium, we call $\kappa_{0}$ the magnetic permeability of the medium and we call the speed-like vector field $\hat{\mathbf{u}} \in \mathbb{R}^{3}$ the optical displacement of the moving dielectric medium. Finally, note that the Maxwell Equations in the medium in the form (9.93) agree with the Fizeau experiment.

Finally, In the case of (9.73), by (9.34) we have

$$
\sum_{r=0}^{3} \sum_{k=0}^{3} K_{r k} \tilde{u}^{k} j^{r} \approx\left(1-|\mathbf{v}|^{2}\right) \rho+\mathbf{v} \cdot \mathbf{j}+\rho \mathbf{v} \cdot \mathbf{u}-\mathbf{u} \cdot \mathbf{j}=\rho-(\mathbf{u}-\mathbf{v}) \cdot(\mathbf{j}-\rho \mathbf{v})
$$

Then, by (9.94) and (9.73) we have

$$
\begin{aligned}
j^{m}-\left(\sum_{r=0}^{3} \sum_{k=0}^{3} K_{r k} \tilde{u}^{k} j^{r}\right) \tilde{u}^{m} \approx j^{m}-\rho u^{m}+(\mathbf{u}-\mathbf{v}) \cdot(\mathbf{j}-\rho \mathbf{v}) u^{m} & \\
& \approx j^{m}-\rho u^{m}+(\mathbf{u}-\mathbf{v}) \cdot(\mathbf{j}-\rho \mathbf{u}) u^{m},
\end{aligned}
$$

so that

$$
\left\{\begin{array}{l}
j^{0}-\left(\sum_{r=0}^{3} \sum_{k=0}^{3} K_{r k} \tilde{u}^{k} j^{r}\right) \tilde{u}^{0} \approx(\mathbf{u}-\mathbf{v}) \cdot(\mathbf{j}-\rho \mathbf{u}) \\
j^{m}-\left(\sum_{r=0}^{3} \sum_{k=0}^{3} K_{r k} \tilde{u}^{k} j^{r}\right) \tilde{u}^{m} \approx j^{m}-\rho u^{m}+(\mathbf{u}-\mathbf{v}) \cdot(\mathbf{j}-\rho \mathbf{u}) u^{m} \quad \forall m=1,2,3 .
\end{array}\right.
$$


On the other hand, by (9.73) and (9.43) we have

$$
\left\{\begin{array}{l}
\sum_{k=0}^{3} F_{0 k} \tilde{u}^{k}=\tilde{u}^{0} F_{00}+\sum_{k=1}^{3} \tilde{u}^{k} F_{0 k} \approx \mathbf{u} \cdot(\mathbf{E}+\mathbf{u} \times \mathbf{B}) \\
\sum_{k=0}^{3} F_{m k} \tilde{u}^{k}=\tilde{u}^{0} F_{m 0}+\sum_{k=1}^{3} \tilde{u}^{k} F_{m k} \approx-(\mathbf{E}+\mathbf{u} \times \mathbf{B}) \quad \forall m=1,2,3 .
\end{array}\right.
$$

However, we have

$$
\left\{\begin{array}{l}
\sum_{r=0}^{3} \sum_{k=0}^{3} K^{0 r} F_{r k} \tilde{u}^{k}=K^{00}\left(\sum_{k=0}^{3} F_{0 k} \tilde{u}^{k}\right)+K^{0 r}\left(\sum_{k=0}^{3} F_{r k} \tilde{u}^{k}\right) \\
\sum_{r=0}^{3} \sum_{k=0}^{3} K^{m r} F_{r k} \tilde{u}^{k}=K^{m 0}\left(\sum_{k=0}^{3} F_{0 k} \tilde{u}^{k}\right)+K^{m r}\left(\sum_{k=0}^{3} F_{r k} \tilde{u}^{k}\right) \quad \forall m=1,2,3 .
\end{array}\right.
$$

Thus, by (9.98) and (9.97), together with (9.34) we have

$$
\left\{\begin{array}{l}
\sum_{r=0}^{3} \sum_{k=0}^{3} K^{0 r} F_{r k} \tilde{u}^{k} \approx Y_{0} \\
\sum_{r=0}^{3} \sum_{k=0}^{3} K^{m r} F_{r k} \tilde{u}^{k} \approx Y^{m} \quad \forall m=1,2,3,
\end{array}\right.
$$

where

$$
\begin{aligned}
\left(Y_{0}, Y_{1}, Y_{2}, Y_{3}\right)= & ((\mathbf{u}-\mathbf{v}) \cdot(\mathbf{E}+\mathbf{u} \times \mathbf{B}),((\mathbf{u}-\mathbf{v}) \cdot(\mathbf{E}+\mathbf{u} \times \mathbf{B})) \mathbf{v}+(\mathbf{E}+\mathbf{u} \times \mathbf{B})) \\
& \approx((\mathbf{u}-\mathbf{v}) \cdot(\mathbf{E}+\mathbf{u} \times \mathbf{B}),((\mathbf{u}-\mathbf{v}) \cdot(\mathbf{E}+\mathbf{u} \times \mathbf{B})) \mathbf{u}+(\mathbf{E}+\mathbf{u} \times \mathbf{B}))
\end{aligned}
$$

Thus, in a cartesian coordinate system (9.31) in the case (9.73) is equivalent to:

$$
\mathbf{j}-\rho \mathbf{u}=\varepsilon(\mathbf{E}+\mathbf{u} \times \mathbf{B}),
$$

that is the usual form of the Ohm's Law in a moving conducting medium. Similarly, we deduce

$$
\left\{\begin{array}{l}
\sum_{r=0}^{3} \sum_{k=0}^{3} K^{0 r} F_{r k}\left(j^{k}-\left(\sum_{r=0}^{3} \sum_{d=0}^{3} K_{r d} \tilde{u}^{d} j^{r}\right) \tilde{u}^{k}\right) \approx Z_{0} \\
\sum_{r=0}^{3} \sum_{k=0}^{3} K^{m r} F_{r k}\left(j^{k}-\left(\sum_{r=0}^{3} \sum_{d=0}^{3} K_{r d} \tilde{u}^{d} j^{r}\right) \tilde{u}^{k}\right) \approx Z^{m} \quad \forall m=1,2,3,
\end{array}\right.
$$

where

$$
\begin{array}{r}
\left(Z_{0}, Z_{1}, Z_{2}, Z_{3}\right)=((\mathbf{u}-\mathbf{v}) \cdot((\mathbf{j}-\rho \mathbf{u}) \times \mathbf{B}),((\mathbf{u}-\mathbf{v}) \cdot((\mathbf{j}-\rho \mathbf{u}) \times \mathbf{B})) \mathbf{v}+((\mathbf{j}-\rho \mathbf{u}) \times \mathbf{B})) \\
\approx((\mathbf{u}-\mathbf{v}) \cdot((\mathbf{j}-\rho \mathbf{u}) \times \mathbf{B}),((\mathbf{u}-\mathbf{v}) \cdot((\mathbf{j}-\rho \mathbf{u}) \times \mathbf{B})) \mathbf{u}+((\mathbf{j}-\rho \mathbf{u}) \times \mathbf{B}))
\end{array}
$$

Thus, in a cartesian coordinate system (9.32) in the case (9.73) is equivalent to:

$$
\mathbf{j}-\rho \mathbf{u}=\varepsilon(\mathbf{E}+\mathbf{u} \times \mathbf{B})-\varsigma(\mathbf{j}-\rho \mathbf{u}) \times \mathbf{B},
$$

that is the usual form of the Ohm's Law, including the Hall effect.

\section{Detailed proves of the stated Theorems, Propositions and Lemmas}

First of all we would like to remind the definitions of the vectors, covectors and covariant and contravariant tensors of second order in $\mathbb{R}^{4}$ : 
Definition 10.1. Let $\mathcal{S}:=\mathcal{S}\left(\mathbb{R}^{4}\right)$ be the group of all smooth non-degenerate invertible transformations from $\mathbb{R}^{4}$ onto $\mathbb{R}^{4}$ having the form

$$
\left\{\begin{array}{l}
x^{\prime 0}=f^{(0)}\left(x^{0}, x^{1}, x^{2}, x^{3}\right), \\
x^{\prime 1}=f^{(1)}\left(x^{0}, x^{1}, x^{2}, x^{3}\right), \\
x^{\prime 2}=f^{(2)}\left(x^{0}, x^{1}, x^{2}, x^{3}\right), \\
x^{\prime 3}=f^{(3)}\left(x^{0}, x^{1}, x^{2}, x^{3}\right) .
\end{array}\right.
$$

We say that a one-component field $a:=a\left(x^{0}, x^{1}, x^{2}, x^{3}\right)$, defined on $\mathbb{R}^{4}$, is a covariant (contravariant) scalar field on the group $\mathcal{S}$, if under the coordinate transformation in the group $\mathcal{S}$ of the form (10.1) this field transforms as:

$$
a^{\prime}=a
$$

Next we say that a four-component field $\left(a^{0}, a^{1}, a^{2}, a^{3}\right)$, defined on $\mathbb{R}^{4}$, is a contravariant four-vector field on the group $\mathcal{S}$, if under the coordinate transformation in the group $\mathcal{S}$ of the form (10.1) every of four components of this field transforms as:

$$
a^{\prime j}=\sum_{k=0}^{3} \frac{\partial f^{(j)}}{\partial x^{k}} a^{k} \quad \forall j=0,1,2,3 .
$$

Next we say that a four-component field $\left(a_{0}, a_{1}, a_{2}, a_{3}\right)$, defined on $\mathbb{R}^{4}$, is a four-covector field on the group $\mathcal{S}$, if under the coordinate transformation in the group $\mathcal{S}$ of the form (10.1) every of four components of this field transforms as:

$$
a_{j}=\sum_{k=0}^{3} \frac{\partial f^{(k)}}{\partial x^{j}} a_{k}^{\prime} \quad \forall j=0,1,2,3 .
$$

Furthermore, we say that a 16 -component field $\left\{a_{m n}\right\}_{m, n=0,1,2,3}$, defined on $\mathbb{R}^{4}$, is a two-times covariant tensor field on the group $\mathcal{S}$, if under the coordinate transformation in the group $\mathcal{S}$ of the form (10.1) every of 16 components of this field transforms as:

$$
a_{m n}=\sum_{j=0}^{3} \sum_{k=0}^{3} \frac{\partial f^{(k)}}{\partial x^{m}} \frac{\partial f^{(j)}}{\partial x^{n}} a_{k j}^{\prime} \quad \forall m, n=0,1,2,3 .
$$

Next we say that a 16 -component field $\left\{a^{m n}\right\}_{m, n=0,1,2,3}$, defined on $\mathbb{R}^{4}$, is a two-times contravariant tensor field on the group $\mathcal{S}$, if under the coordinate transformation in the group $\mathcal{S}$ of the form (10.1) every of 16 components of this field transforms as:

$$
a^{\prime m n}=\sum_{j=0}^{3} \sum_{k=0}^{3} \frac{\partial f^{(m)}}{\partial x^{k}} \frac{\partial f^{(n)}}{\partial x^{j}} a^{k j} \quad \forall m, n=0,1,2,3 .
$$

Then it is well known that for every two contravariant four-vectors $\left(a^{0}, a^{1}, a^{2}, a^{3}\right)$ and $\left(b^{0}, b^{1}, b^{2}, b^{3}\right)$ on $\mathcal{S}$, the 16-component field $\left\{c^{m n}\right\}_{m, n=0,1,2,3}$, defined in every coordinate system by

$$
c^{m n}:=a^{m} b^{n} \quad \forall m, n=0,1,2,3,
$$


is a two-times contravariant tensor on $\mathcal{S}$. Moreover, for every two four-covectors $\left(a_{0}, a_{1}, a_{2}, a_{3}\right)$ and $\left(b_{0}, b_{1}, b_{2}, b_{3}\right)$ on $\mathcal{S}$, the 16 -component field $\left\{c_{m n}\right\}_{m, n=0,1,2,3}$, defined in every coordinate system by

$$
c_{m n}:=a_{m} b_{n} \quad \forall m, n=0,1,2,3,
$$

is a two-times covariant tensor on $\mathcal{S}$. It is also well known that if $\left\{a^{m n}\right\}_{m, n=0,1,2,3}$ is a two times contravariant tensor field on the group $\mathcal{S}$ and if a 16-component field $\left\{b_{m n}\right\}_{m, n=0,1,2,3}$ satisfies

$$
\sum_{k=0}^{3} a^{m k} b_{k n}=\left\{\begin{array}{lll}
1 & \text { if } & m=n \\
0 & \text { if } & m \neq n
\end{array} \quad \forall m, n=0,1,2,3,\right.
$$

then $\left\{b_{m n}\right\}_{m, n=0,1,2,3}$ is a two-times covariant tensor on $\mathcal{S}$. Next it is well known that, given a fourcovector $\left(a_{0}, a_{1}, a_{2}, a_{3}\right)$ a four-vector $\left(b^{0}, b^{1}, b^{2}, b^{3}\right)$, a two-times covariant tensor $\left\{c_{m n}\right\}_{m, n=0,1,2,3}$ and a two-times contravariant tensor $\left\{d^{m n}\right\}_{m, n=0,1,2,3}$ on the group $\mathcal{S}$, the quantities

$$
\sum_{k=0}^{3} a_{k} b^{k} \text { and } \sum_{m=0}^{3} \sum_{n=0}^{3} c_{m n} d^{m n}
$$

are covariant (contravariant) scalars on $\mathcal{S}$, the four-component fields defined by

$$
\left\{\sum_{k=0}^{3} d^{m k} a_{k}\right\}_{m=0,1,2,3} \text { and }\left\{\sum_{k=0}^{3} c_{m k} b^{k}\right\}_{m=0,1,2,3}
$$

are covariant four-vector and contravariant four-covector on $\mathcal{S}$ and moreover, 16-component fields $\left\{\hat{c}^{m n}\right\}_{m, n=0,1,2,3}$ and $\left\{\hat{d}_{m n}\right\}_{m, n=0,1,2,3}$ defined by

$$
\hat{c}^{m n}:=\sum_{k=0}^{3} \sum_{j=0}^{3} d^{m j} d^{n k} c_{j k} \quad \text { and } \quad \hat{d}_{m n}:=\sum_{j=0}^{3} \sum_{k=0}^{3} c_{m j} c_{n k} d^{j k} \quad \forall m, n=0,1,2,3,
$$

are two-times contravariant and two-times covariant tensors on $\mathcal{S}$. Finally, it is well known that, if $a:=a\left(x^{0}, x^{1}, x^{2}, x^{3}\right)$ is a scalar field on the group $\mathcal{S}$, then the four-component field $\left(w_{0}, w_{1}, w_{2}, w_{3}\right)$ defined by:

$$
w_{j}:=\frac{\partial a}{\partial x^{j}} \quad \forall j=0,1,2,3,
$$

is a four-covector field on the group $\mathcal{S}$.

Proof of Proposition 2.1. By (2.8) we obviously deduce that $\left\{\Lambda^{m n}\right\}_{m, n=0,1,2,3}$ is a symmetric twotimes contravariant tensor field. Next, as in (2.5), by (2.4) we have

$$
\sum_{j=0}^{3} \sum_{m=0}^{3} K_{j m} v^{j} v^{m}=1 \quad \forall\left(x^{0}, x^{1}, x^{2}, x^{3}\right) \in \mathbb{R}^{4} .
$$

Moreover, by (2.4) we deduce

$$
\sum_{j=0}^{3} v^{j} w_{j}=\left(\sum_{j=0}^{3} \sum_{k=0}^{3} K^{j k} w_{j} w_{k}\right)^{\frac{1}{2}}
$$


and thus, by (2.8), (10.15) and (2.4) we have

$$
\sum_{j=0}^{3} \Lambda^{m j} w_{j}=0 \quad \forall m=0,1,2,3 .
$$

Next, fix a constant point $\left(x^{0}, x^{1}, x^{2}, x^{3}\right) \in \mathbb{R}^{4}$. Then, since $K^{m n}\left(x^{0}, x^{1}, x^{2}, x^{3}\right)$ has one positive and three negative eigenvalues, by the Sylvester's law of inertia there exist a coordinate system, that we now fix, such that at the fixed point $\left(x^{0}, x^{1}, x^{2}, x^{3}\right)$ we have

$$
\left\{\begin{array}{l}
K^{00}\left(x^{0}, x^{1}, x^{2}, x^{3}\right)=1 \\
K^{j m}\left(x^{0}, x^{1}, x^{2}, x^{3}\right)=-\delta_{j m} \quad \forall 1 \leq j, m \leq 3 \\
K^{0 j}\left(x^{0}, x^{1}, x^{2}, x^{3}\right)=K^{j 0}\left(x^{0}, x^{1}, x^{2}, x^{3}\right)=0 \quad \forall 1 \leq j \leq 3 .
\end{array}\right.
$$

Then, we also have

$$
\left\{\begin{array}{l}
K_{00}\left(x^{0}, x^{1}, x^{2}, x^{3}\right)=1 \\
K_{j m}\left(x^{0}, x^{1}, x^{2}, x^{3}\right)=-\delta_{j m} \quad \forall 1 \leq j, m \leq 3 \\
K_{0 j}\left(x^{0}, x^{1}, x^{2}, x^{3}\right)=K_{j 0}\left(x^{0}, x^{1}, x^{2}, x^{3}\right)=0 \quad \forall 1 \leq j \leq 3 .
\end{array}\right.
$$

In particular, by (2.8) and (10.17) we have at the point $\left(x^{0}, x^{1}, x^{2}, x^{3}\right)$ :

$$
\left\{\begin{array}{l}
\Lambda^{00}\left(x^{0}, x^{1}, x^{2}, x^{3}\right)=\left(v^{0}\left(x^{0}, x^{1}, x^{2}, x^{3}\right)\right)^{2}-1 \\
\Lambda^{j m}\left(x^{0}, x^{1}, x^{2}, x^{3}\right)=\delta_{j m}+v^{j}\left(x^{0}, x^{1}, x^{2}, x^{3}\right) v^{m}\left(x^{0}, x^{1}, x^{2}, x^{3}\right) \quad \forall 1 \leq j, m \leq 3 \\
\Lambda^{0 j}\left(x^{0}, x^{1}, x^{2}, x^{3}\right)=\Lambda^{j 0}\left(x^{0}, x^{1}, x^{2}, x^{3}\right)=v^{0}\left(x^{0}, x^{1}, x^{2}, x^{3}\right) v^{j}\left(x^{0}, x^{1}, x^{2}, x^{3}\right) .
\end{array}\right.
$$

Furthermore, by (10.19), (10.14) and (10.18), using Lemma 11.4 from the Appendix, we deduce that the matrix $\Lambda^{m n}\left(x^{0}, x^{1}, x^{2}, x^{3}\right)$ is degenerate and moreover, it has one vanishing and three positive eigenvalues at the point $\left(x^{0}, x^{1}, x^{2}, x^{3}\right)$. Thus, since $\left(x^{0}, x^{1}, x^{2}, x^{3}\right) \in \mathbb{R}^{4}$ was chosen arbitrary, this completes the proof.

Proof of Proposition 2.2. Consider $\left\{K^{m n}\right\}_{m, n=0,1,2,3}$, given as in (2.17) by

$$
K^{m n}=v^{j} v^{m}-\Lambda^{j m} \quad \forall j, m=0,1,2,3 .
$$

Next, fix a constant point $\left(y^{0}, y^{1}, y^{2}, y^{3}\right) \in \mathbb{R}^{4}$. Then, since $\Lambda^{m n}\left(y^{0}, y^{1}, y^{2}, y^{3}\right)$ has one vanishing and three positive eigenvalues, by the Sylvester's law of inertia there exist a coordinate system, that we now fix, such that at the fixed point $\left(y^{0}, y^{1}, y^{2}, y^{3}\right)$ we have

$$
\left\{\begin{array}{l}
\Lambda^{00}\left(y^{0}, y^{1}, y^{2}, y^{3}\right)=0 \\
\Lambda^{j m}\left(y^{0}, y^{1}, y^{2}, y^{3}\right)=\delta_{j m} \quad \forall 1 \leq j, m \leq 3 \\
\Lambda^{0 j}\left(y^{0}, y^{1}, y^{2}, y^{3}\right)=\Lambda^{j 0}\left(y^{0}, y^{1}, y^{2}, y^{3}\right)=0 \quad \forall 1 \leq j \leq 3 .
\end{array}\right.
$$


Moreover, by (2.15) and by (10.21) in the particular point $\left(y^{0}, y^{1}, y^{2}, y^{3}\right)$ we must have

$$
w_{j}\left(y^{0}, y^{1}, y^{2}, y^{3}\right)=0 \quad \forall j=1,2,3 .
$$

Then by (10.22) and (2.16) we deduce

$$
v^{0}\left(y^{0}, y^{1}, y^{2}, y^{3}\right) w_{0}\left(y^{0}, y^{1}, y^{2}, y^{3}\right)>0,
$$

and in particular, at the point $\left(y^{0}, y^{1}, y^{2}, y^{3}\right)$ we must have

$$
v^{0}\left(y^{0}, y^{1}, y^{2}, y^{3}\right) \neq 0
$$

Thus, by (10.20), (10.21) and (10.24), using Lemma 11.5 from the Appendix we deduce that at the point $\left(y^{0}, y^{1}, y^{2}, y^{3}\right)$ we have

$$
\operatorname{det}\left(\left\{K^{m n}\left(y^{0}, y^{1}, y^{2}, y^{3}\right)\right\}_{m, n=0,1,2,3}\right) \neq 0,
$$

and moreover, the matrix $\left\{K^{m n}\left(y^{0}, y^{1}, y^{2}, y^{3}\right)\right\}_{0 \leq m, n \leq 3}$ necessary has one positive and three negative eigenvalues at the point $\left(y^{0}, y^{1}, y^{2}, y^{3}\right)$. Thus, since a point $\left(y^{0}, y^{1}, y^{2}, y^{3}\right) \in \mathbb{R}^{4}$ was chosen arbitrary, we deduce that the tensor field $\left\{K^{m n}\right\}_{m, n=0,1,2,3}$, given by (10.20) indeed forms a contravariant pseudo-metric and a valid generalized-Lorentz's structure on $\mathbb{R}^{4}$. On the other hand, by (2.15) and (10.20), at every point $\left(x^{0}, x^{1}, x^{2}, x^{3}\right) \in \mathbb{R}^{4}$ we have

$$
\sum_{j=0}^{3} K^{j m} w_{j}=v^{m}\left(\sum_{j=0}^{3} v^{j} w_{j}\right)-\sum_{j=0}^{3} \Lambda^{j m} w_{j}=v^{m}\left(\sum_{j=0}^{3} v^{j} w_{j}\right) \quad \forall m=0,1,2,3 .
$$

Thus, by (10.26) we also infer

$$
\sum_{m=0}^{3}\left(\sum_{j=0}^{3} K^{j m} w_{j}\right) w_{m}=\left(\sum_{j=0}^{3} v^{j} w_{j}\right)^{2}>0 \quad \forall\left(x^{0}, x^{1}, x^{2}, x^{3}\right) \in \mathbb{R}^{4},
$$

so, by (10.27) and (2.16) we get

$$
\sum_{j=0}^{3} v^{j} w_{j}=\left(\sum_{j=0}^{3} \sum_{k=0}^{3} K^{j m} w_{j} w_{k}\right)^{\frac{1}{2}}>0 \quad \forall\left(x^{0}, x^{1}, x^{2}, x^{3}\right) \in \mathbb{R}^{4},
$$

and moreover, by the Definition 2.3, pseudo-metric $K^{j m}$ and the time direction $\left(w_{0}, w_{1}, w_{2}, w_{3}\right)$ are weakly correlated. Finally, by (10.28) and (10.26) we deduce

$$
v^{m}=\left(\sum_{j=0}^{3} \sum_{k=0}^{3} K^{j m} w_{j} w_{k}\right)^{-\frac{1}{2}}\left(\sum_{j=0}^{3} K^{j m} w_{j}\right) \quad \forall m=0,1,2,3 \quad \forall\left(x^{0}, x^{1}, x^{2}, x^{3}\right) \in \mathbb{R}^{4},
$$

This completes the proof. 
Proof of Theorem 2.1. Let

$$
\left\{\begin{array}{l}
x^{0}=f^{(0)}\left(x^{0}, x^{1}, x^{2}, x^{3}\right), \\
x^{\prime 1}=f^{(1)}\left(x^{0}, x^{1}, x^{2}, x^{3}\right), \\
x^{\prime 2}=f^{(2)}\left(x^{0}, x^{1}, x^{2}, x^{3}\right), \\
x^{\prime 3}=f^{(3)}\left(x^{0}, x^{1}, x^{2}, x^{3}\right) .
\end{array}\right.
$$

be the corresponding change of coordinates and assume that in the first coordinate system we have

$$
\left\{\begin{array}{lll}
\Lambda^{00}=0 & \forall\left(x^{0}, x^{1}, x^{2}, x^{3}\right) \in \mathbb{R}^{4} & \\
\Lambda^{0 j}=\Lambda^{j 0}=0 & \forall j=1,2,3 \quad \forall\left(x^{0}, x^{1}, x^{2}, x^{3}\right) \in \mathbb{R}^{4} \\
\Lambda^{m n}:=\delta_{m n} & \forall m, n=1,2,3 & \forall\left(x^{0}, x^{1}, x^{2}, x^{3}\right) \in \mathbb{R}^{4}
\end{array}\right.
$$

and

$$
\left(\frac{\partial \varphi}{\partial x^{0}}, \frac{\partial \varphi}{\partial x^{1}}, \frac{\partial \varphi}{\partial x^{2}}, \frac{\partial \varphi}{\partial x^{3}}\right)\left(x^{0}, x^{1}, x^{2}, x^{3}\right)=(1,0,0,0) \quad \forall\left(x^{0}, x^{1}, x^{2}, x^{3}\right) \in \mathbb{R}^{4} .
$$

However, by (10.4) we have

$$
\frac{\partial \varphi}{\partial x^{j}}=\sum_{k=0}^{3} \frac{\partial f^{(k)}}{\partial x^{j}} \frac{\partial \varphi}{\partial x^{\prime k}} \quad \forall j=0,1,2,3
$$

and by (10.6) we have

$$
\Lambda^{\prime m n}=\sum_{j=0}^{3} \sum_{k=0}^{3} \frac{\partial f^{(m)}}{\partial x^{k}} \frac{\partial f^{(n)}}{\partial x^{j}} \Lambda^{k j} \quad \forall m, n=0,1,2,3 .
$$

Thus, by (10.32) and (10.33) we have

$$
\delta_{0 j}=\sum_{k=0}^{3} \frac{\partial f^{(k)}}{\partial x^{j}} \frac{\partial \varphi}{\partial x^{\prime k}} \quad \forall j=0,1,2,3 .
$$

Moreover, by (10.31) and (10.34) we deduce

$$
\Lambda^{\prime m n}=\sum_{j=1}^{3} \sum_{k=1}^{3} \frac{\partial f^{(m)}}{\partial x^{k}} \frac{\partial f^{(n)}}{\partial x^{j}} \delta_{k j}=\sum_{j=1}^{3} \frac{\partial f^{(m)}}{\partial x^{j}} \frac{\partial f^{(n)}}{\partial x^{j}} \quad \forall m, n=0,1,2,3 .
$$

In particular, by (10.36) we deduce that the following identity

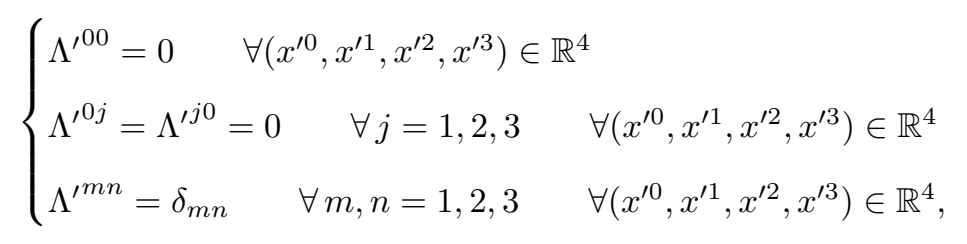

is equivalent to identity:

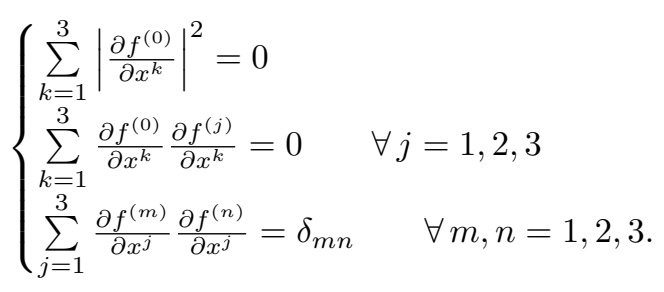


In other words, (10.37) is equivalent to the following:

$$
\left\{\begin{array}{l}
\left(\frac{\partial f^{(0)}}{\partial x^{1}}, \frac{\partial f^{(0)}}{\partial x^{2}}, \frac{\partial f^{(0)}}{\partial x^{3}}\right)=0 \\
\sum_{j=1}^{3} \frac{\partial f^{(j)}}{\partial x^{m}} \frac{\partial f^{(j)}}{\partial x^{n}}=\sum_{j=1}^{3} \frac{\partial f^{(m)}}{\partial x^{j}} \frac{\partial f^{(n)}}{\partial x^{j}}=\delta_{m n} \quad \forall m, n=1,2,3 .
\end{array}\right.
$$

However, using Corollary 11.1 from the Appendix, we deduce that (10.39) is equivalent to the following:

$$
\left\{\begin{array}{l}
x^{\prime 0}=\zeta\left(x^{0}\right), \\
x^{\prime m}=\sum_{j=1}^{3} A_{m j}\left(x^{0}\right) x^{j}+z^{m}\left(x^{0}\right) \quad \forall m=1,2,3,
\end{array}\right.
$$

where $\left\{A_{m n}\left(x^{0}\right)\right\}_{n, m=1,2,3} \in \mathbb{R}^{3 \times 3}$ is a $3 \times 3$-matrix, depending on the coordinate $x^{0}$ only (independent on $\left.\mathbf{x}:=\left(x^{1}, x^{2}, x^{3}\right)\right)$, and satisfying

$$
\sum_{j=1}^{3} A_{m j}\left(x^{0}\right) A_{n j}\left(x^{0}\right)=\sum_{j=1}^{3} A_{j m}\left(x^{0}\right) A_{j n}\left(x^{0}\right)=\delta_{m n} \quad \forall m, n=1,2,3 \quad \forall\left(x^{0}, x^{1}, x^{2}, x^{3}\right) \in \mathbb{R}^{4},
$$

$\zeta\left(x^{0}\right) \in \mathbb{R}$ is depending on the coordinate $x^{0}$ only (independent on $\left.\mathbf{x}:=\left(x^{1}, x^{2}, x^{3}\right)\right)$ and $\mathbf{z}\left(x^{0}\right):=$ $\left(z^{1}\left(x^{0}\right), z^{2}\left(x^{0}\right), z^{3}\left(x^{0}\right)\right) \in \mathbb{R}^{3}$ is a three-dimensional vector field, depending on the coordinate $x^{0}$ only (independent on $\left.\mathbf{x}:=\left(x^{1}, x^{2}, x^{3}\right)\right)$. So, (10.37) is equivalent to (10.40). On the other hand, by (10.35) the following equality

$$
\left(\frac{\partial \varphi}{\partial x^{\prime 0}}, \frac{\partial \varphi}{\partial x^{\prime 1}}, \frac{\partial \varphi}{\partial x^{\prime 2}}, \frac{\partial \varphi}{\partial x^{\prime 3}}\right)\left(x^{\prime 0}, x^{\prime 1}, x^{\prime 2}, x^{\prime 3}\right)=(1,0,0,0) \quad \forall\left(x^{\prime 0}, x^{\prime 1}, x^{\prime 2}, x^{\prime 3}\right) \in \mathbb{R}^{4}
$$

is equivalent to

$$
\delta_{0 j}=\sum_{k=0}^{3} \frac{\partial f^{(k)}}{\partial x^{j}} \delta_{0 k}=\frac{\partial f^{(0)}}{\partial x^{j}} \quad \forall j=0,1,2,3 .
$$

In other words, (10.42) is equivalent to the following:

$$
x^{\prime 0}=x^{0}+c,
$$

where $c$ is a constant independent on $\left(x^{0}, x^{1}, x^{2}, x^{3}\right) \in \mathbb{R}^{4}$. Finally, by the above, (10.37) and (10.42) together are equivalent to (10.40) and (10.44) together. In other words, (10.37) and (10.42) together are equivalent to (2.44). This completes the proof.

Proof of Theorem 2.2. Let

$$
\left\{\begin{array}{l}
x^{\prime 0}=f^{(0)}\left(x^{0}, x^{1}, x^{2}, x^{3}\right), \\
x^{\prime 1}=f^{(1)}\left(x^{0}, x^{1}, x^{2}, x^{3}\right), \\
x^{\prime 2}=f^{(2)}\left(x^{0}, x^{1}, x^{2}, x^{3}\right), \\
x^{\prime 3}=f^{(3)}\left(x^{0}, x^{1}, x^{2}, x^{3}\right) .
\end{array}\right.
$$


be the corresponding change of coordinates and assume that in the first coordinate system we have

$$
\begin{aligned}
& \left\{\begin{array}{lll}
K^{00}=1 \quad \forall\left(x^{0}, x^{1}, x^{2}, x^{3}\right) \in \mathbb{R}^{4} & \\
K^{0 j}=K^{j 0}=0 & \forall j=1,2,3 & \forall\left(x^{0}, x^{1}, x^{2}, x^{3}\right) \in \mathbb{R}^{4} \\
K^{j m}:=-\delta_{j m} & \forall j, m=1,2,3 & \forall\left(x^{0}, x^{1}, x^{2}, x^{3}\right) \in \mathbb{R}^{4},
\end{array}\right. \\
& \left\{\begin{array}{lll}
\Lambda^{00}=0 & \forall\left(x^{0}, x^{1}, x^{2}, x^{3}\right) \in \mathbb{R}^{4} & \\
\Lambda^{0 j}=\Lambda^{j 0}=0 & \forall j=1,2,3 & \forall\left(x^{0}, x^{1}, x^{2}, x^{3}\right) \in \mathbb{R}^{4} \\
\Lambda^{m n}:=\delta_{m n} & \forall m, n=1,2,3 & \forall\left(x^{0}, x^{1}, x^{2}, x^{3}\right) \in \mathbb{R}^{4}
\end{array}\right.
\end{aligned}
$$

and

$$
\left(\frac{\partial \varphi}{\partial x^{0}}, \frac{\partial \varphi}{\partial x^{1}}, \frac{\partial \varphi}{\partial x^{2}}, \frac{\partial \varphi}{\partial x^{3}}\right)\left(x^{0}, x^{1}, x^{2}, x^{3}\right)=(1,0,0,0) \quad \forall\left(x^{0}, x^{1}, x^{2}, x^{3}\right) \in \mathbb{R}^{4} .
$$

In particular, denoting a two-times contravariant tensor $\left\{\Xi^{m n}\right\}_{m, n=0,1,2,3}$, defined by

$$
\Xi^{m n}:=K^{m n}+\Lambda^{m n} \quad \forall m, n=0,1,2,3 \quad \forall\left(x^{0}, x^{1}, x^{2}, x^{3}\right) \in \mathbb{R}^{4},
$$

by (10.46) and (10.47) we have

$$
\left\{\begin{array}{l}
\Xi^{00}=1 \quad \forall\left(x^{0}, x^{1}, x^{2}, x^{3}\right) \in \mathbb{R}^{4} \\
\Xi^{0 j}=\Xi^{j 0}=0 \quad \forall j=1,2,3 \quad \forall\left(x^{0}, x^{1}, x^{2}, x^{3}\right) \in \mathbb{R}^{4} \\
\Xi^{j m}:=0 \quad \forall j, m=1,2,3 \quad \forall\left(x^{0}, x^{1}, x^{2}, x^{3}\right) \in \mathbb{R}^{4} .
\end{array}\right.
$$

However, by (10.6) we have

$$
\Xi^{\prime m n}=\sum_{j=0}^{3} \sum_{k=0}^{3} \frac{\partial f^{(m)}}{\partial x^{k}} \frac{\partial f^{(n)}}{\partial x^{j}} \Xi^{k j} \quad \forall m, n=0,1,2,3 .
$$

Thus, by (10.51) and (10.50) we deduce:

$$
\Xi^{\prime m n}=\frac{\partial f^{(m)}}{\partial x^{0}} \frac{\partial f^{(n)}}{\partial x^{0}} \quad \forall m, n=0,1,2,3 .
$$

In particular, by (10.52) we deduce that the following identity

$$
\left\{\begin{array}{l}
\Xi^{\prime 00}=1 \quad \forall\left(x^{\prime 0}, x^{\prime 1}, x^{\prime 2}, x^{\prime 3}\right) \in \mathbb{R}^{4} \\
\Xi^{\prime 0 j}=\Xi^{\prime j 0}=0 \quad \forall\left(x^{\prime 0}, x^{\prime 1}, x^{\prime 2}, x^{\prime 3}\right) \in \mathbb{R}^{4} \\
\Xi^{\prime m n}=0 \quad \forall m, n=1,2,3 \quad \forall\left(x^{\prime 0}, x^{\prime 1}, x^{\prime 2}, x^{\prime 3}\right) \in \mathbb{R}^{4},
\end{array}\right.
$$

is equivalent to identity:

$$
\left\{\begin{array}{l}
\left(\frac{\partial f^{(m)}}{\partial x^{0}}\right)^{2}=0 \quad \forall m=1,2,3 \\
\frac{\partial f^{(m)}}{\partial x^{0}} \frac{\partial f^{(n)}}{\partial x^{0}}=0 \quad \forall m, n=1,2,3 \\
\frac{\partial f^{(j)}}{\partial x^{0}} \frac{\partial f^{(0)}}{\partial x^{0}} \quad \forall j=1,2,3 \\
\left(\frac{\partial f^{(0)}}{\partial x^{0}}\right)^{2}=1 .
\end{array}\right.
$$


In other words, (10.53) is equivalent to the following:

$$
\left\{\begin{array}{l}
\frac{\partial f^{(m)}}{\partial x^{0}}=0 \quad \forall m=1,2,3 \\
\left(\frac{\partial f^{(0)}}{\partial x^{0}}\right)^{2}=1
\end{array}\right.
$$

On the other hand, using Theorem 2.1 we deduce that

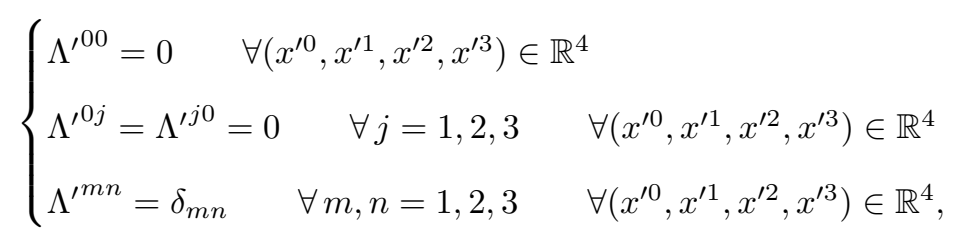

and

$$
\left(\frac{\partial \varphi}{\partial x^{\prime 0}}, \frac{\partial \varphi}{\partial x^{\prime 1}}, \frac{\partial \varphi}{\partial x^{\prime 2}}, \frac{\partial \varphi}{\partial x^{\prime 3}}\right)\left(x^{\prime 0}, x^{\prime 1}, x^{\prime 2}, x^{\prime 3}\right)=(1,0,0,0) \quad \forall\left(x^{\prime 0}, x^{\prime 1}, x^{\prime 2}, x^{\prime 3}\right) \in \mathbb{R}^{4}
$$

together are equivalent to the following relations:

$$
\left\{\begin{array}{l}
x^{\prime 0}=x^{0}+c \\
x^{\prime m}=\sum_{j=1}^{3} A_{m j}\left(x^{0}\right) x^{j}+z^{m}\left(x^{0}\right) \quad \forall m=1,2,3,
\end{array}\right.
$$

where $\left\{A_{m n}\left(x^{0}\right)\right\}_{n, m=1,2,3} \in \mathbb{R}^{3 \times 3}$ is a $3 \times 3$-matrix, depending on the coordinate $x^{0}$ only (independent on $\left.\mathbf{x}:=\left(x^{1}, x^{2}, x^{3}\right)\right)$, and satisfying

$$
\sum_{j=1}^{3} A_{m j}\left(x^{0}\right) A_{n j}\left(x^{0}\right)=\sum_{j=1}^{3} A_{j m}\left(x^{0}\right) A_{j n}\left(x^{0}\right)=\delta_{m n} \quad \forall m, n=1,2,3 \quad \forall\left(x^{0}, x^{1}, x^{2}, x^{3}\right) \in \mathbb{R}^{4},
$$

$c \in \mathbb{R}$ is a constant (independent on $\left.\left(x^{0}, x^{1}, x^{2}, x^{3}\right) \in \mathbb{R}^{4}\right)$ and $\mathbf{z}\left(x^{0}\right):=\left(z^{1}\left(x^{0}\right), z^{2}\left(x^{0}\right), z^{3}\left(x^{0}\right)\right) \in \mathbb{R}^{3}$ is a three-dimensional vector field, depending on the coordinate $x^{0}$ only (independent on $\mathbf{x}:=$ $\left.\left(x^{1}, x^{2}, x^{3}\right)\right)$. Therefore, inserting (10.55) into (10.58) we deduce that, (10.53), (10.56) and (10.57) together are equivalent to (10.58), where $A_{m j}$ and $z^{m}$ are constants (independent on $x^{0}$ ). So, we deduced that

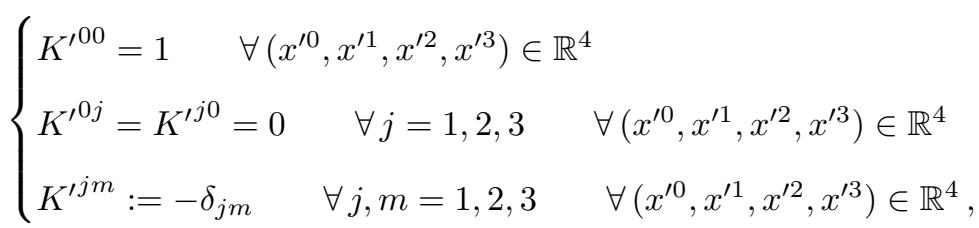

together with (10.56) and (10.57) are equivalent to the following

$$
\left\{\begin{array}{l}
x^{\prime 0}=x^{0}+c^{0}, \\
x^{\prime m}=\sum_{j=1}^{3} B_{m j} x^{j}+c^{m} \quad \forall m=1,2,3,
\end{array}\right.
$$

where $\left\{B_{m n}\right\}_{n, m=1,2,3} \in \mathbb{R}^{3 \times 3}$ is a constant (independent on $\left(x^{0}, x^{1}, x^{2}, x^{3}\right) \in \mathbb{R}^{4}$ ) matrix, satisfying

$$
\sum_{j=1}^{3} B_{m j} B_{n j}=\sum_{j=1}^{3} B_{j m} B_{j n}=\delta_{m n} \quad \forall m, n=1,2,3,
$$


and $\left(c^{0}, c^{1}, c^{2}, c^{3}\right) \in \mathbb{R}^{4}$ is a constant (independent on $\left.\left(x^{0}, x^{1}, x^{2}, x^{3}\right) \in \mathbb{R}^{4}\right)$ vector. This completes the proof.

Proof of Theorem 2.5. First, by Proposition 2.1 we obviously obtain

$$
\sum_{j=0}^{3} \Lambda^{j m} w_{j}=0 \quad \forall m=0,1,2,3,
$$

and

$$
\sum_{j=0}^{3} \sum_{m=0}^{3} J_{j m} r^{j} r^{m}=1 \quad \forall\left(x^{0}, x^{1}, x^{2}, x^{3}\right) \in \mathbb{R}^{4} .
$$

Thus, by (2.122), using Proposition 2.2, we deduce that $\left\{K^{m n}\right\}_{m, n=0,1,2,3}$, given by (2.123), is a symmetric non-degenerate two-times contravariant tensor field, which forms a contravariant pseudometric and a valid generalized-Lorentz's structure on $\mathbb{R}^{4}$. Moreover, by Proposition 2.2 we also have

$$
\begin{gathered}
\sum_{j=0}^{3} K^{m j} w_{j}=\left(\sum_{j=0}^{3} v^{j} w_{j}\right) v^{m} \quad \forall m=0,1,2,3 \quad \forall\left(x^{0}, x^{1}, x^{2}, x^{3}\right) \in \mathbb{R}^{4}, \\
\sum_{j=0}^{3} \sum_{k=0}^{3} K^{j m} w_{j} w_{k}=\left(\sum_{j=0}^{3} v^{j} w_{j}\right)^{2} \quad \forall\left(x^{0}, x^{1}, x^{2}, x^{3}\right) \in \mathbb{R}^{4},
\end{gathered}
$$

and therefore,

$$
v^{m}=\left(\sum_{j=0}^{3} \sum_{k=0}^{3} K^{j m} w_{j} w_{k}\right)^{-\frac{1}{2}}\left(\sum_{j=0}^{3} K^{j m} w_{j}\right) \quad \forall m=0,1,2,3 \quad \forall\left(x^{0}, x^{1}, x^{2}, x^{3}\right) \in \mathbb{R}^{4},
$$

So, $\left(v^{0}, v^{1}, v^{2}, v^{3}\right)$ is a potential of generalized gravity, corresponding to $K^{m n}$ and $\left(w_{0}, w_{1}, w_{2}, w_{3}\right)$. In particular, we have

$$
\operatorname{det}\left(\left\{K^{m n}\right\}_{m, n=0,1,2,3}\right)<0,
$$

and there exists the inverse to $\left\{K^{m n}\right\}_{m, n=0,1,2,3}$ covariant pseudo-metric, which satisfies

$$
\sum_{k=0}^{3} K^{m k} K_{k n}=\left\{\begin{array}{lll}
1 & \text { if } & m=n \\
0 & \text { if } & m \neq n
\end{array} \quad \forall m, n=0,1,2,3 .\right.
$$

Moreover, again, at every point in $\mathbb{R}^{4}$ we also have

$$
\sum_{j=0}^{3} \sum_{m=0}^{3} K_{j m} v^{j} v^{m}=1
$$

Next, fix a constant point $\left(x^{0}, x^{1}, x^{2}, x^{3}\right) \in \mathbb{R}^{4}$. Then, since $\left\{J^{m n}\right\}_{m, n=0,1,2,3}$ has one positive and three negative eigenvalues, by the Sylvester's law of inertia together with (10.64), using Lemma 
11.7 from the Appendix, we deduce that there exist a coordinate system, that we now fix, such that in this coordinate system at the fixed point $\left(x^{0}, x^{1}, x^{2}, x^{3}\right)$ we have

$$
\left\{\begin{array}{l}
J^{00}\left(x^{0}, x^{1}, x^{2}, x^{3}\right)=1 \\
J^{j m}\left(x^{0}, x^{1}, x^{2}, x^{3}\right)=-\delta_{j m} \quad \forall 1 \leq j, m \leq 3 \\
J^{0 j}\left(x^{0}, x^{1}, x^{2}, x^{3}\right)=J^{j 0}\left(x^{0}, x^{1}, x^{2}, x^{3}\right)=0 \quad \forall 1 \leq j \leq 3,
\end{array}\right.
$$

and

$$
\left(r^{0}, r^{1}, r^{2}, r^{3}\right)\left(x^{0}, x^{1}, x^{2}, x^{3}\right)=(1,0,0,0)
$$

Then, we also obviously have

$$
\left\{\begin{array}{l}
J_{00}\left(x^{0}, x^{1}, x^{2}, x^{3}\right)=1 \\
J_{j m}\left(x^{0}, x^{1}, x^{2}, x^{3}\right)=-\delta_{j m} \quad \forall 1 \leq j, m \leq 3 \\
J_{0 j}\left(x^{0}, x^{1}, x^{2}, x^{3}\right)=J_{j 0}\left(x^{0}, x^{1}, x^{2}, x^{3}\right)=0 \quad \forall 1 \leq j \leq 3 .
\end{array}\right.
$$

Moreover, by $(2.121),(10.71)$ and $(10.72)$ we deduce that at the fixed point $\left(x^{0}, x^{1}, x^{2}, x^{3}\right)$ we have

$$
\left\{\begin{array}{l}
\Lambda^{00}\left(x^{0}, x^{1}, x^{2}, x^{3}\right)=0 \\
\Lambda^{j m}\left(x^{0}, x^{1}, x^{2}, x^{3}\right)=\delta_{j m} \quad \forall 1 \leq j, m \leq 3 \\
\Lambda^{0 j}\left(x^{0}, x^{1}, x^{2}, x^{3}\right)=\Lambda^{j 0}\left(x^{0}, x^{1}, x^{2}, x^{3}\right)=0 \quad \forall 1 \leq j \leq 3 .
\end{array}\right.
$$

On the other hand, by (2.120) and (10.72), in the same coordinate system we deduce

$$
w_{j}\left(x^{0}, x^{1}, x^{2}, x^{3}\right)=0 \quad \forall 1 \leq j \leq 3 \quad \text { and } \quad w_{0}\left(x^{0}, x^{1}, x^{2}, x^{3}\right)>0 .
$$

Then, by (10.75) and (2.122) we deduce that

$$
w_{0}\left(x^{0}, x^{1}, x^{2}, x^{3}\right) v^{0}\left(x^{0}, x^{1}, x^{2}, x^{3}\right)=\sum_{j=0}^{3} v^{j}\left(x^{0}, x^{1}, x^{2}, x^{3}\right) w_{j}\left(x^{0}, x^{1}, x^{2}, x^{3}\right)>0 .
$$

In particular, we have

$$
v^{0}\left(x^{0}, x^{1}, x^{2}, x^{3}\right)>0
$$

Furthermore, using Lemma 11.5 from the Appendix, we deduce that $\left\{K_{m n}\right\}_{m, n=0,1,2,3}$ is given at the point $\left(x^{0}, x^{1}, x^{2}, x^{3}\right)$, in the coordinate system, where (10.74) holds, by the following:

$$
\left\{\begin{array}{l}
K_{00}\left(x^{0}, x^{1}, x^{2}, x^{3}\right)=\left(\frac{1}{\left(v^{0}\right)^{2}}-\frac{|\mathbf{v}|^{2}}{\left(v^{0}\right)^{2}}\right)\left(x^{0}, x^{1}, x^{2}, x^{3}\right) \\
K_{j m}\left(x^{0}, x^{1}, x^{2}, x^{3}\right)=-\delta_{j m} \quad \forall 1 \leq j, m \leq 3 \\
K_{0 j}\left(x^{0}, x^{1}, x^{2}, x^{3}\right)=K_{j 0}\left(x^{0}, x^{1}, x^{2}, x^{3}\right)=\left(\frac{v^{j}}{v^{0}}\right)\left(x^{0}, x^{1}, x^{2}, x^{3}\right) \quad \forall 1 \leq j \leq 3,
\end{array}\right.
$$

with $\mathbf{v}:=\left(v^{1}, v^{2}, v^{3}\right)$, and moreover, by Lemma 11.5 from the Appendix, we have

$$
\operatorname{det}\left(\left\{K^{m n}\left(x^{0}, x^{1}, x^{2}, x^{3}\right)\right\}_{m, n=0,1,2,3}\right)=-\left(v^{0}\right)^{2}\left(x^{0}, x^{1}, x^{2}, x^{3}\right)<0 .
$$


Thus, by (10.79) we obviously have

$$
\left(-\operatorname{det}\left(\left\{K_{m n}\right\}_{m, n=0,1,2,3}\right)\right)^{-1}\left(x^{0}, x^{1}, x^{2}, x^{3}\right)=\left(v^{0}\right)^{2}\left(x^{0}, x^{1}, x^{2}, x^{3}\right) .
$$

Therefore, by (10.80) and (10.77) we deduce

$$
\left(-\operatorname{det}\left(\left\{K_{m n}\right\}_{m, n=0,1,2,3}\right)\right)^{-\frac{1}{2}}\left(x^{0}, x^{1}, x^{2}, x^{3}\right)=v^{0}\left(x^{0}, x^{1}, x^{2}, x^{3}\right) .
$$

In particular, by (10.78) and (10.72) we have

$$
\begin{gathered}
\sum_{j=0}^{3} K_{0 j}\left(x^{0}, x^{1}, x^{2}, x^{3}\right) r^{j}\left(x^{0}, x^{1}, x^{2}, x^{3}\right)= \\
K_{00}\left(x^{0}, x^{1}, x^{2}, x^{3}\right) r^{0}\left(x^{0}, x^{1}, x^{2}, x^{3}\right)+\sum_{j=1}^{3} K_{0 j}\left(x^{0}, x^{1}, x^{2}, x^{3}\right) r^{j}\left(x^{0}, x^{1}, x^{2}, x^{3}\right) \\
=\left(\frac{1}{\left(v^{0}\right)^{2}}\left(1-|\mathbf{v}|^{2}\right)\right)\left(x^{0}, x^{1}, x^{2}, x^{3}\right) \\
\quad \sum_{j=0}^{3} K_{m j}\left(x^{0}, x^{1}, x^{2}, x^{3}\right) r^{j}\left(x^{0}, x^{1}, x^{2}, x^{3}\right)= \\
\text { and } \quad \sum_{j=0}^{3} K_{m j}\left(x^{0}, x^{1}, x^{2}, x^{3}\right) r^{j}\left(x^{0}, x^{1}, x^{2}, x^{3}\right)= \\
\quad\left(\frac{1}{v^{0}} v^{m}\right)\left(x^{0}, x^{1}, x^{2}, x^{3}\right) \quad \forall 1 \leq m \leq 3 .
\end{gathered}
$$

Next, if we consider, a covariant four-covector $\left(S_{0}, S_{1}, S_{2}, S_{3}\right)$, defined by:

$$
S_{m}:=\frac{1}{2}\left(\sum_{j=0}^{3} \sum_{k=0}^{3} J^{j k} w_{j} w_{k}\right)^{-\frac{1}{2}}\left(\sum_{j=0}^{3} K_{m j} r^{j}\right)-\frac{1}{2}\left(\sum_{j=0}^{3} v^{j} w_{j}\right)^{-1}\left(\sum_{j=0}^{3} J_{m j} v^{j}\right) \quad \forall 0 \leq m \leq 3,
$$

then by $(10.82),(10.73),(10.75),(10.77)$ and $(10.72)$ we deduce

$$
\begin{aligned}
& S_{0}\left(x^{0}, x^{1}, x^{2}, x^{3}\right)=\left(\frac{1}{2 w_{0}}\left(\frac{1}{\left(v^{0}\right)^{2}}\left(1-|\mathbf{v}|^{2}\right)-1\right)\right)\left(x^{0}, x^{1}, x^{2}, x^{3}\right) \\
& \text { and } \quad S_{m}\left(x^{0}, x^{1}, x^{2}, x^{3}\right)=\left(\frac{1}{w_{0} v^{0}} v^{m}\right)\left(x^{0}, x^{1}, x^{2}, x^{3}\right) \quad \forall 1 \leq m \leq 3 .
\end{aligned}
$$

On the other hand, by (10.78) and (10.73) we have

$$
\left\{\begin{array}{l}
\left(K_{00}-J_{00}\right)\left(x^{0}, x^{1}, x^{2}, x^{3}\right)=\left(\frac{1}{\left(v^{0}\right)^{2}}\left(1-|\mathbf{v}|^{2}\right)-1\right) \\
\left(K_{j m}-J_{j m}\right)\left(x^{0}, x^{1}, x^{2}, x^{3}\right)=0 \quad \forall 1 \leq j, m \leq 3 \\
\left(K_{0 j}-J_{0 j}\right)\left(x^{0}, x^{1}, x^{2}, x^{3}\right)=\left(K_{j 0}-J_{j 0}\right)\left(x^{0}, x^{1}, x^{2}, x^{3}\right)=\frac{w_{0}}{w_{0} v^{0}} v^{j} \quad \forall 1 \leq j \leq 3 .
\end{array}\right.
$$

Thus, by (10.84) and (10.85) we deduce

$$
\left\{\begin{array}{l}
\left(K_{00}-J_{00}\right)\left(x^{0}, x^{1}, x^{2}, x^{3}\right)=2\left(w_{0} S_{0}\right)\left(x^{0}, x^{1}, x^{2}, x^{3}\right) \\
\left(K_{j m}-J_{j m}\right)\left(x^{0}, x^{1}, x^{2}, x^{3}\right)=0 \quad \forall 1 \leq j, m \leq 3 \\
\left(K_{0 j}-J_{0 j}\right)\left(x^{0}, x^{1}, x^{2}, x^{3}\right)=\left(K_{j 0}-J_{j 0}\right)\left(x^{0}, x^{1}, x^{2}, x^{3}\right)=\left(w_{0} S_{j}\right)\left(x^{0}, x^{1}, x^{2}, x^{3}\right) \quad \forall 1 \leq j \leq 3 .
\end{array}\right.
$$


Therefore, inserting (10.75) into (10.86) we deduce

$$
\left\{\begin{array}{l}
\left(K_{00}-J_{00}\right)\left(x^{0}, x^{1}, x^{2}, x^{3}\right)=2\left(w_{0} S_{0}\right)\left(x^{0}, x^{1}, x^{2}, x^{3}\right) \\
\left(K_{j m}-J_{j m}\right)\left(x^{0}, x^{1}, x^{2}, x^{3}\right)=\left(w_{j} S_{m}+w_{m} S_{j}\right) \quad \forall 1 \leq j, m \leq 3 \\
\left(K_{0 j}-J_{0 j}\right)\left(x^{0}, x^{1}, x^{2}, x^{3}\right)=\left(K_{j 0}-J_{j 0}\right)\left(x^{0}, x^{1}, x^{2}, x^{3}\right)=\left(w_{0} S_{j}+w_{j} S_{0}\right) \quad \forall 1 \leq j \leq 3 .
\end{array}\right.
$$

We can rewrite (10.87) in the equivalent form:

$$
K_{j m}\left(x^{0}, x^{1}, x^{2}, x^{3}\right)=J_{j m}\left(x^{0}, x^{1}, x^{2}, x^{3}\right)+\left(w_{j} S_{m}+w_{m} S_{j}\right)\left(x^{0}, x^{1}, x^{2}, x^{3}\right) \quad \forall 0 \leq j, m \leq 3 .
$$

On the other hand, since by (10.71) and (10.75) we have

$$
\left(\sqrt{\sum_{m=0}^{3} \sum_{n=0}^{3} J^{m n} w_{m} w_{n}}\right)\left(x^{0}, x^{1}, x^{2}, x^{3}\right)=w_{0}\left(x^{0}, x^{1}, x^{2}, x^{3}\right),
$$

by (10.73), (10.89), (10.76) and (10.81) we deduce:

$$
\begin{aligned}
&\left(\sqrt{\frac{\left(-\operatorname{det}\left(\left\{J_{m n}\right\}_{m, n=0,1,2,3}\right)\right)\left(\sum_{m=0}^{3} \sum_{n=0}^{3} J^{m n} w_{m} w_{n}\right)}{\left(-\operatorname{det}\left(\left\{K_{m n}\right\}_{m, n=0,1,2,3}\right)\right)}}\right)\left(x^{0}, x^{1}, x^{2}, x^{3}\right) \\
&=\left(\sum_{j=0}^{3} v^{j} w_{j}\right)\left(x^{0}, x^{1}, x^{2}, x^{3}\right)>0 .
\end{aligned}
$$

Next, since $\left(x^{0}, x^{1}, x^{2}, x^{3}\right) \in \mathbb{R}^{4}$ in (10.88) and (10.90) was chosen arbitrary, and moreover since (10.88) and (10.90) are independent on the coordinate system, we deduce that the inverse to $\left\{K^{m n}\right\}_{m, n=0,1,2,3}$ covariant pseudo-metric is given at every point in $\mathbb{R}^{4}$ by the following

$$
K_{j m}=\left(J_{j m}+w_{j} S_{m}+w_{m} S_{j}\right) \quad \forall 0 \leq j, m \leq 3,
$$

where the covariant four-covector $\left(S_{0}, S_{1}, S_{2}, S_{3}\right)$ is defined by (10.83) and, moreover, we have

$$
\left(\sqrt{\frac{\left(-\operatorname{det}\left(\left\{J_{m n}\right\}_{m, n=0,1,2,3}\right)\right)\left(\sum_{m=0}^{3} \sum_{n=0}^{3} J^{m n} w_{m} w_{n}\right)}{\left(-\operatorname{det}\left(\left\{K_{m n}\right\}_{m, n=0,1,2,3}\right)\right)}}\right)=\left(\sum_{j=0}^{3} v^{j} w_{j}\right)>0 .
$$

Finally, by (10.66) and (10.92) we deduce

$$
\sum_{m=0}^{3} \sum_{j=0}^{3} K^{j m} w_{j} w_{m}=\frac{\left(-\operatorname{det}\left(\left\{J_{m n}\right\}_{m, n=0,1,2,3}\right)\right)\left(\sum_{m=0}^{3} \sum_{n=0}^{3} J^{m n} w_{m} w_{n}\right)}{\left(-\operatorname{det}\left(\left\{K_{m n}\right\}_{m, n=0,1,2,3}\right)\right)} .
$$

Proof of Theorem 2.6. As before, we have

$$
r^{m}:=\left(\sum_{j=0}^{3} \sum_{k=0}^{3} J^{j k} w_{j} w_{k}\right)^{-\frac{1}{2}}\left(\sum_{j=0}^{3} J^{m j} w_{j}\right) \quad \forall m=0,1,2,3,
$$


and

$$
\Lambda^{j m}:=r^{j} r^{m}-J^{j m} \quad \forall j, m=0,1,2,3,
$$

so that

$$
\sum_{j=0}^{3} \sum_{m=0}^{3} J_{j m} r^{j} r^{m}=1 \quad \forall\left(x^{0}, x^{1}, x^{2}, x^{3}\right) \in \mathbb{R}^{4} .
$$

Next, consider, an arbitrary covariant four-covector $\left(S_{0}, S_{1}, S_{2}, S_{3}\right)$ such that a two-times covariant tensor field $\left\{K_{m n}\right\}_{m, n=0,1,2,3}$, defined by (2.129) satisfies (2.130) at every point in $\mathbb{R}^{4}$. Then, by (2.130) there exists the inverse to $\left\{K_{m n}\right\}_{m, n=0,1,2,3}$ two-times contravariant symmetric tensor field $\left\{K_{m n}\right\}_{m, n=0,1,2,3}$, which satisfies

$$
\sum_{k=0}^{3} K^{m k} K_{k n}=\left\{\begin{array}{lll}
1 & \text { if } & m=n \\
0 & \text { if } & m \neq n
\end{array} \quad \forall m, n=0,1,2,3 .\right.
$$

Next fix a constant point $\left(x^{0}, x^{1}, x^{2}, x^{3}\right) \in \mathbb{R}^{4}$. Then, by (10.96), since $\left\{J^{m n}\right\}_{m, n=0,1,2,3}$ has one positive and three negative eigenvalues, by the Sylvester's law of inertia together with (10.96), using Lemma 11.7 from the Appendix, we deduce that there exist a coordinate system, that we now fix, such that in this coordinate system at the fixed point $\left(x^{0}, x^{1}, x^{2}, x^{3}\right)$ we have

$$
\left\{\begin{array}{l}
J^{00}\left(x^{0}, x^{1}, x^{2}, x^{3}\right)=1 \\
J^{j m}\left(x^{0}, x^{1}, x^{2}, x^{3}\right)=-\delta_{j m} \quad \forall 1 \leq j, m \leq 3 \\
J^{0 j}\left(x^{0}, x^{1}, x^{2}, x^{3}\right)=J^{j 0}\left(x^{0}, x^{1}, x^{2}, x^{3}\right)=0 \quad \forall 1 \leq j \leq 3,
\end{array}\right.
$$

and

$$
\left(r^{0}, r^{1}, r^{2}, r^{3}\right)\left(x^{0}, x^{1}, x^{2}, x^{3}\right)=(1,0,0,0)
$$

Then, we also obviously have

$$
\left\{\begin{array}{l}
J_{00}\left(x^{0}, x^{1}, x^{2}, x^{3}\right)=1 \\
J_{j m}\left(x^{0}, x^{1}, x^{2}, x^{3}\right)=-\delta_{j m} \quad \forall 1 \leq j, m \leq 3 \\
J_{0 j}\left(x^{0}, x^{1}, x^{2}, x^{3}\right)=J_{j 0}\left(x^{0}, x^{1}, x^{2}, x^{3}\right)=0 \quad \forall 1 \leq j \leq 3 .
\end{array}\right.
$$

Moreover, by (10.95), (10.98) and (10.99) we deduce that at the fixed point $\left(x^{0}, x^{1}, x^{2}, x^{3}\right)$ we have

$$
\left\{\begin{array}{l}
\Lambda^{00}\left(x^{0}, x^{1}, x^{2}, x^{3}\right)=0 \\
\Lambda^{j m}\left(x^{0}, x^{1}, x^{2}, x^{3}\right)=\delta_{j m} \quad \forall 1 \leq j, m \leq 3 \\
\Lambda^{0 j}\left(x^{0}, x^{1}, x^{2}, x^{3}\right)=\Lambda^{j 0}\left(x^{0}, x^{1}, x^{2}, x^{3}\right)=0 \quad \forall 1 \leq j \leq 3 .
\end{array}\right.
$$

Thus, by (10.94) and (10.99), in the same coordinate system we deduce

$$
w_{j}\left(x^{0}, x^{1}, x^{2}, x^{3}\right)=0 \quad \forall 1 \leq j \leq 3 \quad \text { and } \quad w_{0}\left(x^{0}, x^{1}, x^{2}, x^{3}\right)>0,
$$


and by (10.102) and (10.98) we also have

$$
\left(\sqrt{\sum_{m=0}^{3} \sum_{n=0}^{3} J^{m n} w_{m} w_{n}}\right)\left(x^{0}, x^{1}, x^{2}, x^{3}\right)=w_{0}\left(x^{0}, x^{1}, x^{2}, x^{3}\right) .
$$

Next by (10.102), (10.100) and (2.129) we deduce that $\left\{K_{m n}\left(x^{0}, x^{1}, x^{2}, x^{3}\right)\right\}_{m, n=0,1,2,3}$ is given at the point $\left(x^{0}, x^{1}, x^{2}, x^{3}\right)$, in the coordinate system, where (10.100) and (10.99) holds, by the following:

$$
\left\{\begin{array}{l}
K_{00}\left(x^{0}, x^{1}, x^{2}, x^{3}\right)=1+\left(2 w_{0} S_{0}\right)\left(x^{0}, x^{1}, x^{2}, x^{3}\right) \\
K_{j m}\left(x^{0}, x^{1}, x^{2}, x^{3}\right)=-\delta_{j m} \quad \forall 1 \leq j, m \leq 3 \\
K_{0 j}\left(x^{0}, x^{1}, x^{2}, x^{3}\right)=K_{j 0}\left(x^{0}, x^{1}, x^{2}, x^{3}\right)=\left(w_{0} S_{j}\right)\left(x^{0}, x^{1}, x^{2}, x^{3}\right) \quad \forall 1 \leq j \leq 3 .
\end{array}\right.
$$

We rewrite (10.104) as

$$
\left\{\begin{array}{l}
K_{00}\left(x^{0}, x^{1}, x^{2}, x^{3}\right)=1+\left(2 w_{0} S_{0}+w_{0}^{2}|\mathbf{S}|^{2}\right)\left(x^{0}, x^{1}, x^{2}, x^{3}\right)-\left(w_{0}^{2}|\mathbf{S}|^{2}\right)\left(x^{0}, x^{1}, x^{2}, x^{3}\right) \\
K_{j m}\left(x^{0}, x^{1}, x^{2}, x^{3}\right)=-\delta_{j m} \quad \forall 1 \leq j, m \leq 3 \\
K_{0 j}\left(x^{0}, x^{1}, x^{2}, x^{3}\right)=K_{j 0}\left(x^{0}, x^{1}, x^{2}, x^{3}\right)=\left(w_{0} S_{j}\right)\left(x^{0}, x^{1}, x^{2}, x^{3}\right) \quad \forall 1 \leq j \leq 3,
\end{array}\right.
$$

where we denote $\mathbf{S}:=\left(S_{1}, S_{2}, S_{3}\right)$. Thus, by (10.105) for every $\left(h^{0}, h^{1}, h^{2}, h^{3}\right) \in \mathbb{R}^{4}$, with $\mathbf{h}:=$ $\left(h^{1}, h^{2}, h^{3}\right) \in \mathbb{R}^{3}$, we have

$$
\begin{aligned}
\sum_{j=0}^{3} \sum_{m=0}^{3} K_{j m}\left(x^{0}, x^{1}, x^{2}, x^{3}\right) h^{j} h^{m} & = \\
& \left(1+2 w_{0} S_{0}+w_{0}^{2}|\mathbf{S}|^{2}\right)\left(x^{0}, x^{1}, x^{2}, x^{3}\right)\left(h^{0}\right)^{2}-\left(\left|\mathbf{h}-h^{0} w_{0} \mathbf{S}\right|^{2}\right)\left(x^{0}, x^{1}, x^{2}, x^{3}\right) .
\end{aligned}
$$

Thus, if we assume that $\left(1+2 w_{0} S_{0}+w_{0}^{2}|\mathbf{S}|^{2}\right)\left(x^{0}, x^{1}, x^{2}, x^{3}\right)<0$, then by (10.106) we deduce that the matrix $\left\{-K_{m n}\left(x^{0}, x^{1}, x^{2}, x^{3}\right)\right\}_{m, n=0,1,2,3}$ is positively defined, which contradicts to (2.130). On the other hand, if we assume that $\left(1+2 w_{0} S_{0}+w_{0}^{2}|\mathbf{S}|^{2}\right)\left(x^{0}, x^{1}, x^{2}, x^{3}\right)=0$ then, by (10.106) we deduce that $\left(h^{0}, h^{1}, h^{2}, h^{3}\right) \in \mathbb{R}^{4}$, defined by

$$
\left\{\begin{array}{l}
h^{0}=1 \\
h^{j}=w_{0} S_{j} \quad \forall 1 \leq j \leq 3
\end{array}\right.
$$

is an eigenvector of the matrix $\left\{K_{m n}\left(x^{0}, x^{1}, x^{2}, x^{3}\right)\right\}_{m, n=0,1,2,3}$, corresponding to the vanishing eigenvalue, and therefore, the matrix $\left\{K_{m n}\left(x^{0}, x^{1}, x^{2}, x^{3}\right)\right\}_{m, n=0,1,2,3}$ is degenerate, which also contradicts to (2.130). So, (2.130) necessary implies

$$
\left(1+2 w_{0} S_{0}+w_{0}^{2}|\mathbf{S}|^{2}\right)\left(x^{0}, x^{1}, x^{2}, x^{3}\right)>0 .
$$

Therefore, by (10.108), using Lemma 11.5 from the Appendix, we deduce that the inverse to 
$\left\{K_{m n}\left(x^{0}, x^{1}, x^{2}, x^{3}\right)\right\}_{m, n=0,1,2,3}$ matrix $\left\{K^{m n}\left(x^{0}, x^{1}, x^{2}, x^{3}\right)\right\}_{m, n=0,1,2,3}$ is given by the following

$$
\left\{\begin{array}{l}
K^{00}\left(x^{0}, x^{1}, x^{2}, x^{3}\right)=\frac{1}{1+2 w_{0} S_{0}+w_{0}^{2}|\mathbf{S}|^{2}}\left(x^{0}, x^{1}, x^{2}, x^{3}\right) \\
K^{j m}\left(x^{0}, x^{1}, x^{2}, x^{3}\right)=\left(\frac{w_{0}^{2} S_{j} S_{m}}{1+2 w_{0} S_{0}+w_{0}^{2}|\mathbf{S}|^{2}}\right)\left(x^{0}, x^{1}, x^{2}, x^{3}\right)-\delta_{j m} \quad \forall 1 \leq j, m \leq 3 \\
K^{0 j}\left(x^{0}, x^{1}, x^{2}, x^{3}\right)=K^{j 0}\left(x^{0}, x^{1}, x^{2}, x^{3}\right)=\left(\frac{w_{0} S_{j}}{1+2 w_{0} S_{0}+w_{0}^{2}|\mathbf{S}|^{2}}\right)\left(x^{0}, x^{1}, x^{2}, x^{3}\right) \quad \forall 1 \leq j \leq 3,
\end{array}\right.
$$

and moreover, we have

$$
\begin{aligned}
& \left(-\operatorname{det}\left(\left\{K^{m n}\right\}_{m, n=0,1,2,3}\right)\right)^{-1} \\
& \quad=\left(-\operatorname{det}\left(\left\{K_{m n}\right\}_{m, n=0,1,2,3}\right)\right)=\left(1+2 w_{0} S_{0}+w_{0}^{2}|\mathbf{S}|^{2}\right)\left(x^{0}, x^{1}, x^{2}, x^{3}\right)>0 .
\end{aligned}
$$

Then, by (10.110) and (10.109) we infer

$$
\left\{\begin{array}{l}
K^{00}\left(x^{0}, x^{1}, x^{2}, x^{3}\right)=\frac{1}{\left(-\operatorname{det}\left(\left\{K_{m n}\right\}_{m, n=0,1,2,3}\right)\right)}\left(x^{0}, x^{1}, x^{2}, x^{3}\right) \\
K^{j m}\left(x^{0}, x^{1}, x^{2}, x^{3}\right)=\left(\frac{w_{0}^{2} S_{j} S_{m}}{\left(-\operatorname{det}\left(\left\{K_{m n}\right\}_{m, n=0,1,2,3}\right)\right)}\right)\left(x^{0}, x^{1}, x^{2}, x^{3}\right)-\delta_{j m} \quad \forall 1 \leq j, m \leq 3 \\
K^{0 j}\left(x^{0}, x^{1}, x^{2}, x^{3}\right)=K^{j 0}\left(x^{0}, x^{1}, x^{2}, x^{3}\right)=\left(\frac{w_{0} S_{j}}{\left(-\operatorname{det}\left(\left\{K_{m n}\right\}_{m, n=0,1,2,3}\right)\right)}\right)\left(x^{0}, x^{1}, x^{2}, x^{3}\right) \quad \forall 1 \leq j \leq 3 .
\end{array}\right.
$$

Thus, by (10.101), (10.99), (10.103) and (10.100) using (10.111) we deduce

$$
\begin{array}{r}
K^{m n}\left(x^{0}, x^{1}, x^{2}, x^{3}\right)=v^{m}\left(x^{0}, x^{1}, x^{2}, x^{3}\right) v^{n}\left(x^{0}, x^{1}, x^{2}, x^{3}\right)-\Lambda^{m n}\left(x^{0}, x^{1}, x^{2}, x^{3}\right) \\
\forall m, n=0,1,2,3,
\end{array}
$$

where the contravariant vector field $\left(v^{0}, v^{1}, v^{2}, v^{3}\right)$ is given by

$$
\begin{array}{r}
v^{m}:=\left(\sqrt{\frac{-\operatorname{det}\left(\left\{J_{k n}\right\}_{k, n=0,1,2,3}\right)}{-\operatorname{det}\left(\left\{K_{k n}\right\}_{k, n=0,1,2,3}\right)}}\right)\left(\left(\sqrt{\sum_{j=0}^{3} \sum_{k=0}^{3} J^{j k} w_{j} w_{k}}\right)\left(\sum_{j=0}^{3} \Lambda^{m j} S_{j}\right)+r^{m}\right) \\
\forall m=0,1,2,3 .
\end{array}
$$

Moreover, by (10.113), (10.99), (10.101) and (10.102) we obtain

$$
\left(\sum_{j=0}^{3} v^{j} w_{j}\right)\left(x^{0}, x^{1}, x^{2}, x^{3}\right)=\left(w_{0} \sqrt{\frac{-\operatorname{det}\left(\left\{J_{m n}\right\}_{m, n=0,1,2,3}\right)}{-\operatorname{det}\left(\left\{K_{m n}\right\}_{m, n=0,1,2,3}\right)}}\right)\left(x^{0}, x^{1}, x^{2}, x^{3}\right)>0 .
$$

Next, since $\left(x^{0}, x^{1}, x^{2}, x^{3}\right) \in \mathbb{R}^{4}$ in (10.112) and (10.114) was chosen arbitrary, and moreover since (10.112) and the left hand side of (10.114) are independent on the coordinate system, we deduce that at every point we have

$$
K^{m n}=v^{m} v^{n}-\Lambda_{m n} \quad \forall m, n=0,1,2,3 .
$$

where the contravariant vector field $\left(v^{0}, v^{1}, v^{2}, v^{3}\right)$ is given by (10.113) and moreover, we have

$$
\left(\sum_{j=0}^{3} v^{j} w_{j}\right)>0
$$


Thus we can apply Theorem 2.5 to complete the proof.

Proof of Proposition 2.3. Differentiating (2.58) in Definition 2.13 gives,

$$
\begin{aligned}
& \frac{\partial x^{\prime m}}{\partial x^{n}}= \\
& B_{m n}\left(\sum_{k=0}^{3} w_{k} x^{k}\right)+\left(\sum_{j=0}^{3} \frac{d B_{m j}}{d s}\left(\sum_{k=0}^{3} w_{k} x^{k}\right) x^{j}+\frac{d z^{m}}{d s}\left(\sum_{k=0}^{3} w_{k} x^{k}\right)\right) w_{n} \quad \forall m, n=0,1,2,3 .
\end{aligned}
$$

However, differentiating the identity

$$
B^{-1}(s) \cdot B(s)=I d_{4 \times 4} \quad \forall s \in \mathbb{R},
$$

gives

$$
\frac{d B^{-1}}{d s}(s) \cdot B(s)+B^{-1}(s) \cdot \frac{d B}{d s}(s)=0 \quad \forall s \in \mathbb{R},
$$

that implies,

$$
\frac{d B^{-1}}{d s}(s)=-B^{-1}(s) \cdot \frac{d B}{d s}(s) \cdot B^{-1}(s) \quad \forall s \in \mathbb{R} .
$$

Therefore, by (2.60) in Definition 2.13 together with (10.120) we deduce:

$$
\begin{aligned}
& \frac{d}{d s}\left(\sum_{j=0}^{3} w_{j}\left(\left\{B^{-1}\right\}_{j m}(s)\right)\right)=\sum_{j=0}^{3} w_{j}\left(\left\{\frac{d B^{-1}}{d s}\right\}_{j m}(s)\right)= \\
& -\sum_{j=0}^{3} w_{j}\left(\left\{B^{-1}(s) \cdot \frac{d B}{d s}(s) \cdot B^{-1}(s)\right\}_{j m}(s)\right)= \\
& -\sum_{k=0}^{3} \sum_{j=0}^{3} w_{j}\left(\left\{B^{-1} \cdot \frac{d B}{d s}\right\}_{j k}(s)\right)\left(\left\{B^{-1}\right\}_{k m}(s)\right)=0 \quad \forall m=0,1,2,3, \quad \forall s \in \mathbb{R} .
\end{aligned}
$$

In particular,

$$
\sum_{j=0}^{3} w_{j}\left(\left\{B^{-1}\right\}_{j m}(s)\right)=\sum_{j=0}^{3} w_{j}\left(\left\{B^{-1}\right\}_{j m}(0)\right) \quad \forall m=0,1,2,3, \quad \forall s \in \mathbb{R} .
$$

Then, using (10.117) and (10.122), together with (2.60) and (2.61) in Definition 2.13 we deduce:

$$
\begin{gathered}
\sum_{m=0}^{3} w_{m}^{\prime} \frac{\partial x^{\prime m}}{\partial x^{n}}=\sum_{m=0}^{3} \sum_{j=0}^{3} w_{j}\left(\left\{B^{-1}\right\}_{j m}(0)\right) \frac{\partial x^{\prime m}}{\partial x^{n}}=\sum_{m=0}^{3} \sum_{j=0}^{3} w_{j}\left(\left\{B^{-1}\right\}_{j m}\left(\sum_{k=0}^{3} w_{k} x^{k}\right)\right) \frac{\partial x^{\prime m}}{\partial x^{n}} \\
=\sum_{m=0}^{3} \sum_{j=0}^{3} w_{j}\left(\left\{B^{-1}\right\}_{j m}\left(\sum_{k=0}^{3} w_{k} x^{k}\right)\right) B_{m n}\left(\sum_{k=0}^{3} w_{k} x^{k}\right) \\
+\sum_{m=0}^{3} \sum_{j=0}^{3} w_{j}\left(\left\{B^{-1}\right\}_{j m}\left(\sum_{k=0}^{3} w_{k} x^{k}\right)\right)\left(\sum_{r=0}^{3} \frac{d B_{m r}}{d s}\left(\sum_{k=0}^{3} w_{k} x^{k}\right) x^{r}+\frac{d z^{m}}{d s}\left(\sum_{k=0}^{3} w_{k} x^{k}\right)\right) w_{n} \\
=w_{n} \quad \forall n=0,1,2,3 .
\end{gathered}
$$


Moreover, by (2.57) and (2.59) in Definition 2.13 we deduce

$$
\begin{aligned}
\sum_{m=0}^{3} \sum_{n=0}^{3} M^{m n} w_{m}^{\prime} w_{n}^{\prime}=\sum_{m=0}^{3} \sum_{n=0}^{3} M^{m n}\left(\sum_{j=0}^{3} w_{j}\left(\left\{B^{-1}\right\}_{j m}(0)\right)\right) & \left(\sum_{k=0}^{3} w_{k}\left(\left\{B^{-1}\right\}_{k n}(0)\right)\right) \\
& =\sum_{j=0}^{3} \sum_{k=0}^{3} M^{j k} w_{j} w_{k}=1 .
\end{aligned}
$$

This completes the proof.

Proof of Proposition 2.4. By Definition 2.13 we have,

$$
x^{\prime m}:=\sum_{j=0}^{3} B_{m j}\left(\sum_{k=0}^{3} w_{k} x^{k}\right) x^{j}+z^{m}\left(\sum_{k=0}^{3} w_{k} x^{k}\right) \quad \forall m=0,1,2,3,
$$

where $B(s):=\left\{B_{m j}(s)\right\}_{m, j=0,1,2,3}: \mathbb{R} \rightarrow \mathbb{R}^{4 \times 4}$ and $\left(z^{0}(s), z^{1}(s), z^{2}(s), z^{3}(s)\right): \mathbb{R} \rightarrow \mathbb{R}^{4}$ satisfy

$$
\begin{aligned}
& M^{m n}= \sum_{j=0}^{3} \sum_{k=0}^{3} B_{m j}(s) B_{n k}(s) M^{j k} \quad \forall m, n=0,1,2,3, \quad \forall s \in \mathbb{R}, \\
& \sum_{j=0}^{3} w_{j}\left(\left\{B^{-1} \cdot \frac{d B}{d s}\right\}_{j m}(s)\right)=0 \quad \forall m=0,1,2,3, \quad \forall s \in \mathbb{R},
\end{aligned}
$$

and

$$
\sum_{m=0}^{3} \sum_{j=0}^{3} w_{j}\left(\left\{B^{-1}\right\}_{j m}(s)\right) \frac{d z^{m}}{d s}(s)=0 \quad \forall s \in \mathbb{R} .
$$

However, by (2.72) in Proposition 2.3 there exists a constant $C \in \mathbb{R}$ such that we have

$$
\left(\sum_{k=0}^{3} w_{k}^{\prime} x^{\prime k}\right)=C+\left(\sum_{k=0}^{3} w_{k} x^{k}\right)
$$

Thus, by (10.125) and (10.129) we deduce

$$
x^{\prime m}=\sum_{j=0}^{3} B_{m j}\left(\sum_{k=0}^{3} w_{k}^{\prime} x^{\prime k}-C\right) x^{j}+z^{m}\left(\sum_{k=0}^{3} w_{k}^{\prime} x^{\prime k}-C\right) \quad \forall m=0,1,2,3,
$$

that we rewrite as:

$$
\begin{array}{r}
x^{m}=\sum_{j=0}^{3}\left\{B^{-1}\right\}_{m j}\left(\sum_{k=0}^{3} w_{k}^{\prime} x^{\prime k}-C\right) x^{\prime j}-\sum_{j=0}^{3}\left\{B^{-1}\right\}_{m j}\left(\sum_{k=0}^{3} w_{k}^{\prime} x^{\prime k}-C\right) z^{j}\left(\sum_{k=0}^{3} w_{k}^{\prime} x^{\prime k}-C\right) \\
\forall m=0, \ldots, 3 .
\end{array}
$$

Thus, denoting $B^{\prime}(s):=\left\{B_{m j}^{\prime}(s)\right\}_{m, j=0,1,2,3}: \mathbb{R} \rightarrow \mathbb{R}^{4 \times 4}$ and $\left(z^{\prime 0}(s), z^{\prime 1}(s), z^{\prime 2}(s), z^{\prime 3}(s)\right): \mathbb{R} \rightarrow \mathbb{R}^{4}$, given by

$$
\left\{\begin{array}{l}
B^{\prime}(s)=B^{-1}(s-C) \\
z^{\prime m}(s)=-\sum_{j=0}^{3}\left\{B^{-1}\right\}_{m j}(s-C) z^{j}(s-C) \quad \forall m=0, \ldots, 3
\end{array}\right.
$$


by (10.131) we infer

$$
x^{m}=\sum_{j=0}^{3} B_{m j}^{\prime}\left(\sum_{k=0}^{3} w_{k}^{\prime} x^{\prime k}\right) x^{\prime j}+z^{\prime m}\left(\sum_{k=0}^{3} w_{k}^{\prime} x^{\prime k}\right) \quad \forall m=0, \ldots, 3 .
$$

However, by (10.126) and (10.132) we deduce

$$
M^{m n}=\sum_{j=0}^{3} \sum_{k=0}^{3} B_{m j}^{\prime}(s) B_{n k}^{\prime}(s) M^{j k} \quad \forall m, n=0,1,2,3, \quad \forall s \in \mathbb{R},
$$

Moreover, by (10.132) and (2.70) in Proposition 2.3 we deduce

$$
\sum_{j=0}^{3} w_{j}^{\prime}\left(\left\{B^{\prime-1} \cdot \frac{d B^{\prime}}{d s}\right\}_{j m}(s)\right)=\sum_{m=0}^{3} \sum_{j=0}^{3} w_{j} \frac{d}{d s}\left\{B^{-1}\right\}_{j m}(s-C) \quad \forall m=0,1,2,3, \quad \forall s \in \mathbb{R},
$$

and

$$
\begin{aligned}
\sum_{m=0}^{3} \sum_{j=0}^{3} w_{j}^{\prime}\left(\left\{B^{\prime-1}\right\}_{j m}(s)\right) \frac{d z^{\prime m}}{d s}(s)= & \\
& \sum_{m=0}^{3} \sum_{j=0}^{3} w_{m} \frac{d}{d s}\left(-\sum_{k=0}^{3}\left\{B^{-1}\right\}_{m k}(s-C) z^{k}(s-C)\right) \\
= & \sum_{m=0}^{3} \sum_{j=0}^{3} w_{m}\left(-\sum_{k=0}^{3} \frac{d}{d s}\left(\left\{B^{-1}\right\}_{m k}(s-C)\right) z^{k}(s-C)\right) \\
& +\sum_{m=0}^{3} \sum_{j=0}^{3} w_{m}\left(-\sum_{k=0}^{3}\left\{B^{-1}\right\}_{m k}(s-C) \frac{d z^{k}}{d s}(s-C)\right) \quad \forall s \in \mathbb{R} .
\end{aligned}
$$

On the other hand, as in (10.120) we have:

$$
\frac{d B^{-1}}{d s}(s-C)=-B^{-1}(s-C) \cdot \frac{d B}{d s}(s-C) \cdot B^{-1}(s-C) \quad \forall s \in \mathbb{R} .
$$

Thus, by (10.135) and (10.137) together, using (10.127) we deduce

$$
\sum_{j=0}^{3} w_{j}^{\prime}\left(\left\{B^{\prime-1} \cdot \frac{d B^{\prime}}{d s}\right\}_{j m}(s)\right)=0 \quad \forall m=0,1,2,3, \quad \forall s \in \mathbb{R} .
$$

Finally, by (10.136) and (10.137) together, using (10.127) and (10.128) we deduce

$$
\sum_{m=0}^{3} \sum_{j=0}^{3} w_{j}^{\prime}\left(\left\{B^{\prime-1}\right\}_{j m}(s)\right) \frac{d z^{\prime m}}{d s}(s)=0 \quad \forall s \in \mathbb{R} .
$$

This completes the proof.

Lemma 10.1. Given a constant vector $\left(w_{0}, w_{1}, w_{2}, w_{3}\right) \in \mathbb{R}^{4}$, satisfying

$$
\left(w_{0}\right)^{2}-\sum_{j=1}^{3}\left(w_{j}\right)^{2}=1
$$

assume that that the change of coordinate system $\left(x^{0}, x^{1}, x^{2}, x^{3}\right) \rightarrow\left(x^{\prime 0}, x^{\prime 1}, x^{\prime 2}, x^{\prime 3}\right)$ belongs to the class $P L\left(w_{0}, w_{1}, w_{2}, w_{3}\right)$. Next, as before, let $\left(w^{\prime 0}, w_{1}^{\prime}, w_{2}^{\prime}, w_{3}^{\prime}\right) \in \mathbb{R}^{4}$ be defined as

$$
w_{m}^{\prime}=\sum_{j=0}^{3} w_{j}\left(\left\{B^{-1}\right\}_{j m}(0)\right) \quad \forall m=0,1,2,3, \quad \forall s \in \mathbb{R},
$$


where $B(s):=\left\{B_{m j}(s)\right\}_{m, j=0,1,2,3}: \mathbb{R} \rightarrow \mathbb{R}^{4 \times 4}$ be as in (2.58). Furthermore, consider another change of coordinate system $\left(x^{\prime 0}, x^{\prime 1}, x^{\prime 2}, x^{\prime 3}\right) \rightarrow\left(x^{\prime \prime 0}, x^{\prime \prime 1}, x^{\prime \prime 2}, x^{\prime \prime 3}\right)$, belonging to the class $P L\left(w_{0}^{\prime}, w_{1}^{\prime}, w_{2}^{\prime}, w_{3}^{\prime}\right)$.

Then, the composition of the above two changes of coordinate systems:

$$
\left(x^{0}, x^{1}, x^{2}, x^{3}\right) \rightarrow\left(x^{\prime \prime 0}, x^{\prime \prime 1}, x^{\prime \prime 2}, x^{\prime \prime 3}\right)=\left(x^{0}, x^{1}, x^{2}, x^{3}\right) \rightarrow\left(x^{\prime 0}, x^{\prime 1}, x^{\prime 2}, x^{\prime 3}\right) \rightarrow\left(x^{\prime \prime 0}, x^{\prime \prime 1}, x^{\prime \prime 2}, x^{\prime \prime 3}\right)
$$

also belongs to the class $P L\left(w_{0}, w_{1}, w_{2}, w_{3}\right)$.

Proof of Lemma 10.1. As before, assume that $\left(w_{0}, w_{1}, w_{2}, w_{3}\right) \in \mathbb{R}^{4}, B(s):=\left\{B_{m j}(s)\right\}_{m, j=0,1,2,3}$ : $\mathbb{R} \rightarrow \mathbb{R}^{4 \times 4}$ and $\left(z^{0}(s), z^{1}(s), z^{2}(s), z^{3}(s)\right): \mathbb{R} \rightarrow \mathbb{R}^{4}$ satisfy

$$
\begin{gathered}
\sum_{j=0}^{3} \sum_{m=0}^{3} M^{j m} w_{j} w_{m}=1, \\
M^{m n}=\sum_{j=0}^{3} \sum_{k=0}^{3} B_{m j}(s) B_{n k}(s) M^{j k} \quad \forall m, n=0,1,2,3, \quad \forall s \in \mathbb{R}, \\
\sum_{j=0}^{3} w_{j}\left(\left\{B^{-1} \cdot \frac{d B}{d s}\right\}_{j m}(s)\right)=0 \quad \forall m=0,1,2,3, \quad \forall s \in \mathbb{R},
\end{gathered}
$$

and

$$
\sum_{m=0}^{3} \sum_{j=0}^{3} w_{j}\left(\left\{B^{-1}\right\}_{j m}(s)\right) \frac{d z^{m}}{d s}(s)=0 \quad \forall s \in \mathbb{R} .
$$

Then, consider

$$
x^{\prime m}=\sum_{j=0}^{3} B_{m j}\left(\sum_{k=0}^{3} w_{k} x^{k}\right) x^{j}+z^{m}\left(\sum_{k=0}^{3} w_{k} x^{k}\right) \quad \forall m=0,1,2,3 .
$$

Furthermore, let $\left(w_{0}^{\prime}, w_{1}^{\prime}, w_{2}^{\prime}, w_{3}^{\prime}\right) \in \mathbb{R}^{4}$ be defined as

$$
w_{m}^{\prime}=\sum_{j=0}^{3} w_{j}\left(\left\{B^{-1}\right\}_{j m}(0)\right) \quad \forall m=0,1,2,3, \quad \forall s \in \mathbb{R},
$$

and assume that $B^{\prime}(s):=\left\{B_{m j}^{\prime}(s)\right\}_{m, j=0,1,2,3}: \mathbb{R} \rightarrow \mathbb{R}^{4 \times 4}$ and $\left(z^{\prime 0}(s), z^{11}(s), z^{\prime 2}(s), z^{\prime 3}(s)\right): \mathbb{R} \rightarrow$ $\mathbb{R}^{4}$ satisfy

$$
\begin{array}{cc}
M^{m n}=\sum_{j=0}^{3} \sum_{k=0}^{3} B_{m j}^{\prime}(s) B_{n k}^{\prime}(s) M^{j k} & \forall m, n=0,1,2,3, \quad \forall s \in \mathbb{R}, \\
\sum_{j=0}^{3} w_{j}^{\prime}\left(\left\{B^{\prime-1} \cdot \frac{d B^{\prime}}{d s}\right\}_{j m}(s)\right)=0 \quad \forall m=0,1,2,3, \quad \forall s \in \mathbb{R},
\end{array}
$$

and

$$
\sum_{m=0}^{3} \sum_{j=0}^{3} w_{j}^{\prime}\left(\left\{B^{\prime-1}\right\}_{j m}(s)\right) \frac{d z^{\prime m}}{d s}(s)=0 \quad \forall s \in \mathbb{R} .
$$

Then, consider

$$
x^{\prime \prime m}=\sum_{j=0}^{3} B_{m j}^{\prime}\left(\sum_{k=0}^{3} w_{k}^{\prime} x^{\prime k}\right) x^{\prime j}+z^{\prime m}\left(\sum_{k=0}^{3} w_{k}^{\prime} x^{\prime k}\right) \quad \forall m=0,1,2,3 .
$$


However by (2.72) in Proposition 2.3 we have

$$
\left(\sum_{k=0}^{3} w_{k}^{\prime} x^{\prime k}\right)=C+\left(\sum_{k=0}^{3} w_{k} x^{k}\right)
$$

In particular, (2.72) in Proposition 2.3 together with (10.146) in the case $x^{j}=0$, with the help of (10.147), give us the following:

$$
\sum_{m=0}^{3} \sum_{r=0}^{3}\left(\left\{B^{-1}\right\}_{r m}(0)\right) w_{r} z^{m}(0)=C
$$

Therefore, by (10.153) we rewrite (10.152) as:

$$
\sum_{m=0}^{3} w_{m}^{\prime} x^{\prime m}=\sum_{j=0}^{3} w_{j} x^{j}+\sum_{m=0}^{3} \sum_{r=0}^{3}\left(\left\{B^{-1}\right\}_{r m}(0)\right) w_{r} z^{m}(0) .
$$

Thus, by (10.151), (10.146) and (10.154) we have

$$
x^{\prime \prime m}=\sum_{j=0}^{3} B_{m j}^{\prime \prime}\left(\sum_{k=0}^{3} w_{k} x^{k}\right) x^{j}+z^{\prime \prime m}\left(\sum_{k=0}^{3} w_{k} x^{k}\right) \quad \forall m=0,1,2,3,
$$

with $B^{\prime \prime}(s):=\left\{B_{m j}^{\prime \prime}(s)\right\}_{m, j=0,1,2,3}: \mathbb{R} \rightarrow \mathbb{R}^{4 \times 4}$, given by

$$
B_{m j}^{\prime \prime}(s):=\sum_{q=0}^{3} B_{m q}^{\prime}\left(\tau_{s}\right) B_{q j}(s) \quad \forall m, j=0,1,2,3 \quad \forall s \in \mathbb{R},
$$

and with

$$
z^{\prime \prime m}(s):=\sum_{j=0}^{3} B_{m j}^{\prime}\left(\tau_{s}\right) z^{j}(s)+z^{\prime m}\left(\tau_{s}\right) \quad \forall m=0,1,2,3 \quad \forall s \in \mathbb{R},
$$

where we denote

$$
\tau_{s}:=s+\sum_{d=0}^{3} \sum_{r=0}^{3}\left(\left\{B^{-1}\right\}_{r d}(0)\right) w_{r} z^{d}(0) .
$$

In particular, by (10.143), (10.148) and (10.156) we deduce

$$
M^{m n}=\sum_{j=0}^{3} \sum_{k=0}^{3} B_{m j}^{\prime \prime}(s) B_{n k}^{\prime \prime}(s) M^{j k} \quad \forall m, n=0,1,2,3, \quad \forall s \in \mathbb{R} .
$$

Moreover, by (10.156) we have

$$
\begin{array}{r}
\sum_{j=0}^{3} w_{j}\left(\left\{B^{\prime \prime-1} \cdot \frac{d B^{\prime \prime}}{d s}\right\}_{j m}(s)\right)=\sum_{j=0}^{3} w_{j}\left(\left\{B^{-1}(s) \cdot B^{\prime-1}\left(\tau_{s}\right) \cdot\left(\frac{d B^{\prime}}{d s}\left(\tau_{s}\right) \cdot B(s)\right)\right\}_{j m}\right) \\
+\sum_{j=0}^{3} w_{j}\left(\left\{B^{-1}(s) \cdot B^{\prime-1}\left(\tau_{s}\right) \cdot\left(B^{\prime}\left(\tau_{s}\right) \cdot \frac{d B}{d s}(s)\right)\right\}_{j m}\right)= \\
\sum_{j=0}^{3} w_{j}\left(\left\{B^{-1}(s) \cdot B^{\prime-1}\left(\tau_{s}\right) \cdot\left(\frac{d B^{\prime}}{d s}\left(\tau_{s}\right) \cdot B(s)\right)\right\}_{j m}\right)+\sum_{j=0}^{3} w_{j}\left(\left\{B^{-1}(s) \cdot \frac{d B}{d s}(s)\right\}_{j m}\right) \\
\forall m=0,1,2,3, \quad \forall s \in \mathbb{R} .
\end{array}
$$


Thus, by (2.68) and (2.70) in Proposition 2.3 and (10.144), (10.149), we rewrite (10.160) as:

$$
\begin{aligned}
& \sum_{j=0}^{3} w_{j}\left(\left\{B^{\prime \prime-1} \cdot \frac{d B^{\prime \prime}}{d s}\right\}_{j m}(s)\right)= \\
& \sum_{j=0}^{3} w_{j}\left(\left\{B^{-1}(s) \cdot B^{\prime-1}\left(\tau_{s}\right) \cdot\left(\frac{d B^{\prime}}{d s}\left(\tau_{s}\right) \cdot B(s)\right)\right\}_{j m}\right)+\sum_{j=0}^{3} w_{j}\left(\left\{B^{-1}(s) \cdot \frac{d B}{d s}(s)\right\}_{j m}\right) \\
& =\sum_{j=0}^{3} w_{j}\left(\left\{B^{-1}(0) \cdot B^{\prime-1}\left(\tau_{s}\right) \cdot\left(\frac{d B^{\prime}}{d s}\left(\tau_{s}\right) \cdot B(s)\right)\right\}_{j m}\right)= \\
& \sum_{j=0}^{3} w_{j}^{\prime}\left(\left\{B^{\prime-1}\left(\tau_{s}\right) \cdot\left(\frac{d B^{\prime}}{d s}\left(\tau_{s}\right) \cdot B(s)\right)\right\}_{j m}\right)=0 \quad \forall m=0,1,2,3, \quad \forall s \in \mathbb{R} . \quad
\end{aligned}
$$

Similarly, by (2.68) and (2.70) in Proposition 2.3 together with (10.145), (10.150), (10.156), (10.149) and (10.157), using (10.158) we obtain:

$$
\begin{gathered}
\sum_{m=0}^{3} \sum_{j=0}^{3} w_{j}\left(\left\{B^{\prime \prime-1}\right\}_{j m}(s)\right) \frac{d z^{\prime \prime m}}{d s}(s)= \\
\sum_{m=0}^{3} \sum_{j=0}^{3} w_{j}\left(\left\{B^{-1}(s) \cdot B^{\prime-1}\left(\tau_{s}\right)\right\}_{j m}\right) \frac{d}{d s}\left(\sum_{k=0}^{3} B_{m k}^{\prime}\left(\tau_{s}\right) z^{k}(s)+z^{\prime m}\left(\tau_{s}\right)\right) \\
=\sum_{m=0}^{3} \sum_{j=0}^{3} w_{j}\left(\left\{B^{-1}(s) \cdot B^{\prime-1}\left(\tau_{s}\right)\right\}_{j m}\right)\left(\sum_{k=0}^{3} \frac{d B_{m k}^{\prime}}{d s}\left(\tau_{s}\right) z^{k}(s)\right) \\
+\sum_{m=0}^{3} \sum_{j=0}^{3} w_{j}\left(\left\{B^{-1}(s) \cdot B^{\prime-1}\left(\tau_{s}\right)\right\}_{j m}\right)\left(\sum_{k=0}^{3} B_{m k}^{\prime}\left(\tau_{s}\right) \frac{d z^{k}}{d s}(s)\right) \\
\quad+\sum_{m=0}^{3} \sum_{j=0}^{3} w_{j}\left(\left\{B^{-1}(s) \cdot B^{\prime-1}\left(\tau_{s}\right)\right\}_{j m}\right) \frac{d z^{\prime m}}{d s}\left(\tau_{s}\right) \\
=\sum_{j=0}^{3} \sum_{k=0}^{3} w_{j}^{\prime}\left(\left\{B^{\prime-1}\left(\tau_{s}\right) \cdot \frac{d B^{\prime}}{d s}\left(\tau_{s}\right)\right\}_{j k}\right) z^{k}(s)+\sum_{k=0}^{3} \sum_{j=0}^{3} w_{j}\left(\left\{B^{-1}(s)\right\}_{j k}\right) \frac{d z^{k}}{d s}(s) \\
+\sum_{m=0}^{3} \sum_{j=0}^{3} w_{j}^{\prime}\left(\left\{B^{\prime-1}\left(\tau_{s}\right)\right\}_{j m}\right) \frac{d z^{\prime m}}{d s}\left(\tau_{s}\right)=0 \quad \forall s \in \mathbb{R} . \quad(10.1
\end{gathered}
$$

This completes the proof.

Lemma 10.2. For every constant vector $\left(w_{0}, w_{1}, w_{2}, w_{3}\right) \in \mathbb{R}^{4}$, satisfying

$$
\sum_{j=0}^{3} \sum_{m=0}^{3} M^{j m} w_{j} w_{m}=1
$$

the classes $L\left(\left(w_{0}, w_{1}, w_{2}, w_{3}\right),(1,0,0,0)\right)$ and $L\left((1,0,0,0),\left(w_{0}, w_{1}, w_{2}, w_{3}\right)\right)$ are not empty. Moreover, for every transformation in $L\left(\left(w_{0}, w_{1}, w_{2}, w_{3}\right),(1,0,0,0)\right)$, the inverse transformation belongs to $L\left((1,0,0,0),\left(w_{0}, w_{1}, w_{2}, w_{3}\right)\right)$.

Proof of Lemma 10.2. Follows by Lemma 11.7. 
Corollary 10.1. For every given two fixed constant vectors $\left(w_{0}, w_{1}, w_{2}, w_{3}\right) \in \mathbb{R}^{4},\left(w_{0}^{\prime}, w_{1}^{\prime}, w_{2}^{\prime}, w_{3}^{\prime}\right) \in$ $\mathbb{R}^{4}$, satisfying

$$
\left\{\begin{array}{l}
\sum_{j=0}^{3} \sum_{m=0}^{3} M^{j m} w_{j} w_{m}=1 \\
\sum_{j=0}^{3} \sum_{m=0}^{3} M^{j m} w_{j}^{\prime} w_{m}^{\prime}=1,
\end{array}\right.
$$

the class $L\left(\left(w_{0}, w_{1}, w_{2}, w_{3}\right),\left(w_{0}^{\prime}, w_{1}^{\prime}, w_{2}^{\prime}, w_{3}^{\prime}\right)\right)$ is not empty. Moreover, for every transformation in $L\left(\left(w_{0}, w_{1}, w_{2}, w_{3}\right),\left(w_{0}^{\prime}, w_{1}^{\prime}, w_{2}^{\prime}, w_{3}^{\prime}\right)\right)$, the inverse transformation belongs to $L\left(\left(w_{0}^{\prime}, w_{1}^{\prime}, w_{2}^{\prime}, w_{3}^{\prime}\right),\left(w_{0}, w_{1}, w_{2}, w_{3}\right)\right)$.

Lemma 10.3. Assume that the new coordinate system is obtained from some old Pseudo-Lorentzian system by a Lorentz's transformation. Then the new coordinate system is also a Pseudo-Lorentzian system.

Proof of Lemma 10.3. Consider a ancillary contravariant pseudo-metric $\left\{L^{m n}\right\}_{m, n=0,1,2,3}$, given in the old coordinate system by

$$
\begin{cases}L^{00}=1 & \\ L^{0 j}=L^{j 0}=0 & \forall j=1,2,3 \\ L^{j m}:=-\delta_{j m} & \forall j, m=1,2,3 .\end{cases}
$$

Note that in general this pseudo-metric can differ from pseudo-metric $\left\{K^{m n}\right\}_{m, n=0,1,2,3}$. Then, since the old system is Pseudo-Lorentzian, there exists a covector, which in this system is equal to some constant (independent on the point $\left.\left(x^{0}, x^{1}, x^{2}, x^{3}\right) \in \mathbb{R}^{4}\right)$ vector $\left(w_{0}, w_{1}, w_{2}, w_{3}\right) \in \mathbb{R}^{4}$ satisfying

$$
\sum_{m=0}^{3} \sum_{j=0}^{3} L^{m j} w_{m} w_{j}=1
$$

and such that in the old system we have

$$
\Lambda^{m n}=-L^{m n}+\left(\sum_{k=0}^{3} L^{m k} w_{k}\right)\left(\sum_{k=0}^{3} L^{n k} w_{k}\right) \quad \forall 0 \leq m, n \leq 3
$$

and at the same time in the old coordinate system we have

$$
\left(\frac{\partial \varphi}{\partial x^{0}}, \frac{\partial \varphi}{\partial x^{1}}, \frac{\partial \varphi}{\partial x^{2}}, \frac{\partial \varphi}{\partial x^{3}}\right)\left(x^{0}, x^{1}, x^{2}, x^{3}\right)=\left(w_{0}, w_{1}, w_{2}, w_{3}\right) \quad \forall\left(x^{0}, x^{1}, x^{2}, x^{3}\right) \in \mathbb{R}^{4}
$$

On the other hand, since the new system is obtained from the old one by some Lorentz's transformation, that are also necessarily linear, in the new coordinate system we also have

$$
\left\{\begin{array}{l}
L^{\prime 00}=1 \\
L^{\prime 0 j}=L^{\prime j 0}=0 \quad \forall j=1,2,3 \\
L^{\prime j m}:=-\delta_{j m} \quad \forall j, m=1,2,3,
\end{array}\right.
$$


and moreover, the covector $\left(w_{0}^{\prime}, w_{1}^{\prime}, w_{2}^{\prime}, w_{3}^{\prime}\right) \in \mathbb{R}^{4}$ also in the new system is equal to some constant vector in $\mathbb{R}^{4}$ (independent on the point $\left.\left(x^{\prime 0}, x^{\prime 1}, x^{\prime 2}, x^{\prime 3}\right) \in \mathbb{R}^{4}\right)$. Finally, by covariance of (10.166), (10.167) and (10.168), in the new system obviously we have

$$
\begin{gathered}
\sum_{m=0}^{3} \sum_{j=0}^{3} L^{\prime m j} w_{m} w_{j}^{\prime}=1, \\
\Lambda^{\prime m n}=-L^{\prime m n}+\left(\sum_{k=0}^{3} L^{\prime m k} w_{k}^{\prime}\right)\left(\sum_{k=0}^{3} L^{\prime n k} w_{k}^{\prime}\right) \quad \forall 0 \leq m, n \leq 3,
\end{gathered}
$$

and

$$
\left(\frac{\partial \varphi}{\partial x^{\prime 0}}, \frac{\partial \varphi}{\partial x^{\prime 1}}, \frac{\partial \varphi}{\partial x^{\prime 2}}, \frac{\partial \varphi}{\partial x^{\prime 3}}\right)\left(x^{\prime 0}, x^{\prime 1}, x^{\prime 2}, x^{\prime 3}\right)=\left(w_{0}^{\prime}, w_{1}^{\prime}, w_{2}^{\prime}, w_{3}^{\prime}\right) \quad \forall\left(x^{\prime 0}, x^{\prime 1}, x^{\prime 2}, x^{\prime 3}\right) \in \mathbb{R}^{4} .
$$

Thus, using (10.169), we deduce by (10.170), (10.171) and (10.172) that the new system is also Pseudo-Lorentzian.

Proof of Theorem 2.3. Consider a Pseudo-Lorentzian coordinate system, so that

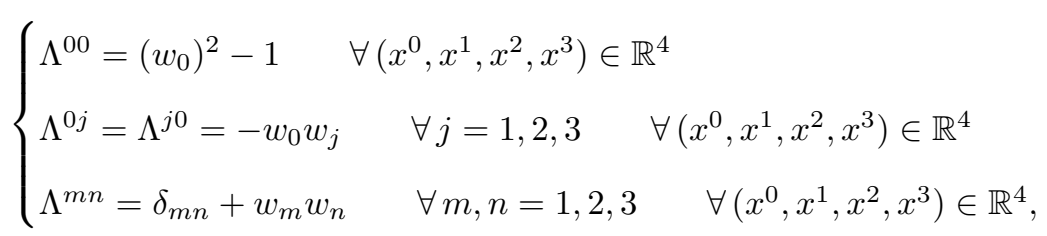

and at the same time in the same coordinate system we have

$$
\left(\frac{\partial \varphi}{\partial x^{0}}, \frac{\partial \varphi}{\partial x^{1}}, \frac{\partial \varphi}{\partial x^{2}}, \frac{\partial \varphi}{\partial x^{3}}\right)\left(x^{0}, x^{1}, x^{2}, x^{3}\right)=\left(w_{0}, w_{1}, w_{2}, w_{3}\right) \quad \forall\left(x^{0}, x^{1}, x^{2}, x^{3}\right) \in \mathbb{R}^{4},
$$

where $\left(w_{0}, w_{1}, w_{2}, w_{3}\right) \in \mathbb{R}^{4}$ is some constant (independent on the point $\left.\left(x^{0}, x^{1}, x^{2}, x^{3}\right) \in \mathbb{R}^{4}\right)$ vector, satisfying

$$
\left(w_{0}\right)^{2}-\sum_{j=1}^{3}\left(w_{j}\right)^{2}=1
$$

Next, again let $\left\{L^{m n}\right\}_{m, n=0,1,2,3}$ be an ancillary contravariant pseudo-metric, satisfying

$$
\left\{\begin{array}{l}
L^{00}=1 \\
L^{0 j}=L^{j 0}=0 \quad \forall j=1,2,3 \\
L^{j m}:=-\delta_{j m} \quad \forall j, m=1,2,3
\end{array}\right.
$$

in the given coordinate system. Then, by Lemma 11.7, there exists a constant non-degenerate matrix $\left\{A_{m n}\right\}_{0 \leq m, n \leq 3} \in \mathbb{R}^{4 \times 4}$, such that $\operatorname{det}\left(\left\{A_{m n}\right\}_{0 \leq m, n \leq 3}\right) \neq 0$, and if we consider a matrix $\left\{L^{\prime m n}\right\}_{0 \leq m, n \leq 3} \in \mathbb{R}^{4 \times 4}$, defined by

$$
L^{\prime m n}:=\sum_{j=0}^{3} \sum_{k=0}^{3} A_{m k} A_{n j} L^{k j} \quad \forall m, n=0,1,2,3,
$$


and a vector $\left(w^{\prime 0}, w^{\prime 1}, w^{\prime 2}, w^{\prime 3}\right) \in \mathbb{R}^{4}$, defined by

$$
w^{\prime j}:=\sum_{k=0}^{3} A_{j k} w^{k} \quad \forall j=0,1,2,3
$$

then we have:

$$
\left\{\begin{array}{l}
L^{\prime 00}=1 \\
L^{\prime j m}=-\delta_{j m} \quad \forall 1 \leq j, m \leq 3 \\
L^{\prime 0 j}=L^{\prime j 0}=0 \quad \forall 1 \leq j \leq 3,
\end{array}\right.
$$

and

$$
\left(w^{\prime 0}, w^{\prime 1}, w^{\prime 2}, w^{\prime 3}\right)=(1,0,0,0) .
$$

Obviously matrix $\left\{A_{m n}\right\}_{0 \leq m, n \leq 3} \in \mathbb{R}^{4 \times 4}$ represents some Lorentz's transformation leading to a new system. However, by (10.173), (10.174) and (10.175) we have

$$
\begin{gathered}
\sum_{m=0}^{3} \sum_{j=0}^{3} L^{m j} w_{m} w_{j}=1, \\
\Lambda^{m n}=-L^{m n}+\left(\sum_{k=0}^{3} L^{m k} w_{k}\right)\left(\sum_{k=0}^{3} L^{n k} w_{k}\right) \quad \forall 0 \leq m, n \leq 3 .
\end{gathered}
$$

and

$$
\left(\frac{\partial \varphi}{\partial x^{0}}, \frac{\partial \varphi}{\partial x^{1}}, \frac{\partial \varphi}{\partial x^{2}}, \frac{\partial \varphi}{\partial x^{3}}\right)\left(x^{0}, x^{1}, x^{2}, x^{3}\right)=\left(w_{0}, w_{1}, w_{2}, w_{3}\right) \quad \forall\left(x^{0}, x^{1}, x^{2}, x^{3}\right) \in \mathbb{R}^{4} .
$$

Thus, again by a covariance in the new system we also observe:

$$
\begin{gathered}
\sum_{m=0}^{3} \sum_{j=0}^{3} L^{\prime m j} w_{m} w_{j}^{\prime}=1, \\
\Lambda^{\prime m n}=-L^{\prime m n}+\left(\sum_{k=0}^{3} L^{\prime m k} w_{k}^{\prime}\right)\left(\sum_{k=0}^{3} L^{\prime n k} w_{k}^{\prime}\right) \quad \forall 0 \leq m, n \leq 3 .
\end{gathered}
$$

and

$$
\left(\frac{\partial \varphi}{\partial x^{\prime 0}}, \frac{\partial \varphi}{\partial x^{\prime 1}}, \frac{\partial \varphi}{\partial x^{\prime 2}}, \frac{\partial \varphi}{\partial x^{\prime 3}}\right)\left(x^{\prime 0}, x^{\prime 1}, x^{\prime 2}, x^{\prime 3}\right)=\left(w_{0}^{\prime}, w_{1}^{\prime}, w_{2}^{\prime}, w_{3}^{\prime}\right) \quad \forall\left(x^{\prime 0}, x^{\prime 1}, x^{\prime 2}, x^{\prime 3}\right) \in \mathbb{R}^{4},
$$

Therefore, inserting (10.180) into (10.186) gives

$$
\left(\frac{\partial \varphi}{\partial x^{\prime 0}}, \frac{\partial \varphi}{\partial x^{\prime 1}}, \frac{\partial \varphi}{\partial x^{\prime 2}}, \frac{\partial \varphi}{\partial x^{\prime 3}}\right)\left(x^{\prime 0}, x^{\prime 1}, x^{\prime 2}, x^{\prime 3}\right)=(1,0,0,0) \quad \forall\left(x^{\prime 0}, x^{\prime 1}, x^{\prime 2}, x^{\prime 3}\right) \in \mathbb{R}^{4},
$$

and inserting (10.180) together with (10.179) into (10.185) gives,

$$
\left\{\begin{array}{l}
\Lambda^{\prime 00}=0 \\
\Lambda^{\prime 0 j}=\Lambda^{\prime j 0}=0 \quad \forall j=1,2,3 \\
\Lambda^{\prime j m}=\delta_{j m} \quad \forall j, m=1,2,3 .
\end{array}\right.
$$


Therefore, by (10.187) and (10.188) the new system is cartesian and obtained from the old system by some Lorentz's transformation. Thus, since the inverse transform to Lorentz's transformation is also a Lorentz's transformation, we deduce that the original system (old) is obtained from the cartesian system (new) by some Lorentz's transformation.

Conversely, if any new system is obtained from the old cartesian coordinate system by Lorentz's transformation, then since every cartesian system is also a Pseudo-Lorentzian coordinate system, by Lemma 10.3 we deduce that the new system is also a Pseudo-Lorentzian coordinate system.

Proof of Theorem 2.4. Indeed, assume that the transformation in (2.67) is of class $P L\left(\left(w_{0}, w_{1}, w_{2}, w_{3}\right)\right)$. Then, obviously there exists another constant vector $\left(w_{0}^{\prime}, w_{1}^{\prime}, w_{2}^{\prime}, w_{3}^{\prime}\right) \in \mathbb{R}^{4}$, satisfying

$$
\left(w_{0}^{\prime}\right)^{2}-\sum_{j=1}^{3}\left(w_{j}^{\prime}\right)^{2}=1,
$$

and such that the transformation in $(2.67)$ is of class $P L\left(\left(w_{0}, w_{1}, w_{2}, w_{3}\right) ;\left(w_{0}^{\prime}, w_{1}^{\prime}, w_{2}^{\prime}, w_{3}^{\prime}\right)\right)$. Then by Corollary 2.8 there exists three other changes of coordinate system $\left(x^{0}, x^{1}, x^{2}, x^{3}\right) \rightarrow\left(x^{\prime 0}, x^{\prime 1}, x^{\prime 2}, x^{\prime 3}\right)$, belonging to the class $L\left(\left(w_{0}, w_{1}, w_{2}, w_{3}\right) ;(1,0,0,0)\right)$, $\left(x^{\prime 0}, x^{\prime 1}, x^{\prime 2}, x^{\prime 3}\right) \rightarrow\left(x^{\prime \prime 0}, x^{\prime \prime 1}, x^{\prime \prime 2}, x^{\prime \prime 3}\right)$, belonging to the class $P L((1,0,0,0) ;(1,0,0,0))$ and $\left(x^{\prime \prime 0}, x^{\prime \prime 1}, x^{\prime \prime 2}, x^{\prime \prime 3}\right) \rightarrow\left(x^{\prime \prime \prime 0}, x^{\prime \prime \prime 1}, x^{\prime \prime \prime 2}, x^{\prime \prime \prime 3}\right)$, belonging to the class $L\left(\left((1,0,0,0) ;\left(w_{0}^{\prime}, w_{1}^{\prime}, w_{2}^{\prime}, w_{3}^{\prime}\right)\right)\right.$, so that the original transformation $\left(x^{0}, x^{1}, x^{2}, x^{3}\right) \rightarrow\left(x^{\prime \prime \prime}, x^{\prime \prime \prime}, x^{\prime \prime \prime 2}, x^{\prime \prime \prime}\right)$ is a composition of the above three changes of coordinate systems:

$$
\begin{aligned}
\left(x^{0}, x^{1}, x^{2}, x^{3}\right) & \rightarrow\left(x^{\prime \prime \prime}, x^{\prime \prime \prime 1}, x^{\prime \prime \prime 2}, x^{\prime \prime \prime 3}\right) \\
\left(x^{0}, x^{1}, x^{2}, x^{3}\right) & \rightarrow\left(x^{\prime 0}, x^{\prime 1}, x^{\prime 2}, x^{\prime 3}\right) \rightarrow\left(x^{\prime \prime 0}, x^{\prime \prime 1}, x^{\prime \prime 2}, x^{\prime \prime 3}\right) \rightarrow\left(x^{\prime \prime \prime}, x^{\prime \prime \prime 1}, x^{\prime \prime \prime 2}, x^{\prime \prime \prime 3}\right) .
\end{aligned}
$$

However, since the original system with coordinates $\left(x^{0}, x^{1}, x^{2}, x^{3}\right)$ is Pseudo-Lorntzian and since, the change $\left(x^{0}, x^{1}, x^{2}, x^{3}\right) \rightarrow\left(x^{\prime 0}, x^{\prime 1}, x^{\prime 2}, x^{\prime 3}\right)$, belongs to the class $L\left(\left(w_{0}, w_{1}, w_{2}, w_{3}\right) ;(1,0,0,0)\right)$, by Lemma 10.3 the system with coordinates $\left(x^{\prime 0}, x^{\prime 1}, x^{\prime 2}, x^{\prime 3}\right)$ is Pseudo-Lorntzian, and moreover, we have

$$
\left(\frac{\partial \varphi}{\partial x^{\prime 0}}, \frac{\partial \varphi}{\partial x^{\prime 1}}, \frac{\partial \varphi}{\partial x^{\prime 2}}, \frac{\partial \varphi}{\partial x^{\prime 3}}\right)\left(x^{\prime 0}, x^{\prime 1}, x^{\prime 2}, x^{\prime 3}\right)=(1,0,0,0) \quad \forall\left(x^{\prime 0}, x^{\prime 1}, x^{\prime 2}, x^{\prime 3}\right) \in \mathbb{R}^{4},
$$

and thus also

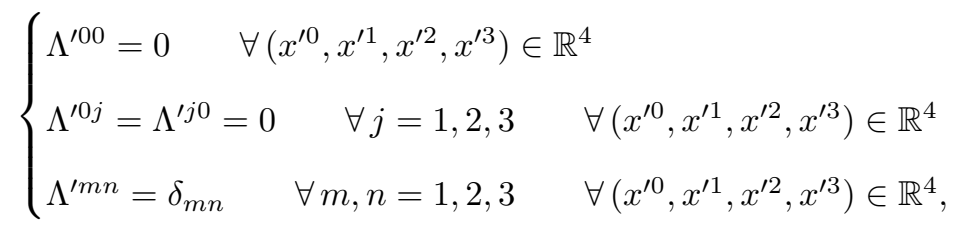

so that the system with coordinates $\left(x^{\prime 0}, x^{\prime 1}, x^{\prime 2}, x^{\prime 3}\right)$ is cartesian. Therefore, since the change $\left(x^{\prime 0}, x^{\prime 1}, x^{\prime 2}, x^{\prime 3}\right) \rightarrow\left(x^{\prime \prime 0}, x^{\prime \prime 1}, x^{\prime \prime 2}, x^{\prime \prime 3}\right)$, belongs to the class $P L((1,0,0,0) ;(1,0,0,0))$, by Corollary 
2.7 we deduce that the system with coordinates $\left(x^{\prime \prime 0}, x^{\prime \prime 1}, x^{\prime \prime 2}, x^{\prime \prime 3}\right)$ is also cartesian, and thus in particular, the system with coordinates $\left(x^{\prime \prime 0}, x^{\prime \prime 1}, x^{\prime \prime 2}, x^{\prime \prime 3}\right)$ is Pseudo-Lorentzian. Finally, since the change $\left(x^{\prime \prime 0}, x^{\prime \prime 1}, x^{\prime \prime 2}, x^{\prime \prime 3}\right) \rightarrow\left(x^{\prime \prime \prime 0}, x^{\prime \prime \prime 1}, x^{\prime \prime \prime 2}, x^{\prime \prime \prime 3}\right)$ is a Lorentian transformation, and since the system with coordinates $\left(x^{\prime \prime 0}, x^{\prime \prime 1}, x^{\prime \prime 2}, x^{\prime \prime 3}\right)$ is Pseudo-Lorentzian, by Lemma 10.3 we deduce that the system with coordinates $\left(x^{\prime \prime \prime}, x^{\prime \prime \prime 1}, x^{\prime \prime \prime 2}, x^{\prime \prime \prime 3}\right)$ is also Pseudo-Lorntzian.

Conversely, assume that the system with coordinates $\left(x^{\prime \prime \prime}, x^{\prime \prime \prime 1}, x^{\prime \prime \prime 2}, x^{\prime \prime \prime 3}\right)$ is Pseudo-Lorntzian. Then, by Theorem 2.3, there exists two Lorentz's transformations $\left(x^{0}, x^{1}, x^{2}, x^{3}\right) \rightarrow\left(x^{\prime 0}, x^{\prime 1}, x^{\prime 2}, x^{\prime 3}\right)$ and $\left(x^{\prime \prime 0}, x^{\prime \prime 1}, x^{\prime \prime 2}, x^{\prime \prime 3}\right) \rightarrow\left(x^{\prime \prime \prime}, x^{\prime \prime \prime}, x^{\prime \prime \prime 2}, x^{\prime \prime \prime 3}\right)$, so that the both systems with coordinates $\left(x^{\prime 0}, x^{\prime 1}, x^{\prime 2}, x^{\prime 3}\right)$ and $\left(x^{\prime \prime 0}, x^{\prime \prime 1}, x^{\prime \prime 2}, x^{\prime \prime 3}\right)$ are cartesian. Then, again by Corollary 2.7 , the change $\left(x^{\prime 0}, x^{\prime 1}, x^{\prime 2}, x^{\prime 3}\right) \rightarrow\left(x^{\prime \prime 0}, x^{\prime \prime 1}, x^{\prime \prime 2}, x^{\prime \prime 3}\right)$, belongs to the class $P L((1,0,0,0) ;(1,0,0,0))$. However, since the system with coordinates $\left(x^{\prime 0}, x^{\prime 1}, x^{\prime 2}, x^{\prime 3}\right)$ is cartesian, by $(2.65)$ we deduce that the transformation $\left(x^{0}, x^{1}, x^{2}, x^{3}\right) \rightarrow\left(x^{\prime 0}, x^{\prime 1}, x^{\prime 2}, x^{\prime 3}\right)$ also belongs to the class $P L\left(\left(w_{0}, w_{1}, w_{2}, w_{3}\right) ;(1,0,0,0)\right)$. Therefore, by Proposition 2.5 we obtain that the transformation $\left(x^{0}, x^{1}, x^{2}, x^{3}\right) \rightarrow\left(x^{\prime \prime 0}, x^{\prime \prime 1}, x^{\prime \prime 2}, x^{\prime \prime 3}\right)$ also belongs to the class $P L\left(\left(w_{0}, w_{1}, w_{2}, w_{3}\right) ;(1,0,0,0)\right)$. On the other hand, since the change $\left(x^{\prime \prime 0}, x^{\prime \prime 1}, x^{\prime \prime 2}, x^{\prime \prime 3}\right) \rightarrow\left(x^{\prime \prime \prime 0}, x^{\prime \prime \prime 1}, x^{\prime \prime \prime 2}, x^{\prime \prime \prime 3}\right)$ is a Lorentz's transformation, it also belongs to the class $P L((1,0,0,0))$. Then, by Corollary 2.6 together with Proposition 2.5, we finally deduce that the composed transformation $\left(x^{0}, x^{1}, x^{2}, x^{3}\right) \rightarrow\left(x^{\prime \prime \prime 0}, x^{\prime \prime \prime 1}, x^{\prime \prime \prime 2}, x^{\prime \prime \prime 3}\right)$ given through:

$$
\left(x^{0}, x^{1}, x^{2}, x^{3}\right) \rightarrow\left(x^{\prime \prime 0}, x^{\prime \prime 1}, x^{\prime \prime 2}, x^{\prime \prime 3}\right) \rightarrow\left(x^{\prime \prime \prime 0}, x^{\prime \prime \prime 1}, x^{\prime \prime \prime 2}, x^{\prime \prime \prime 3}\right)
$$

belongs to the class $P L\left(\left(w_{0}, w_{1}, w_{2}, w_{3}\right)\right)$.

Proof of Proposition 3.1. For every smooth scalar classical function with compact support $\xi\left(x^{0}, x^{1}, x^{2}, x^{3}\right) \in C_{c}^{\infty}\left(\mathbb{R}^{4}\right)$, by $(3.7)$ we have

$$
\begin{aligned}
& \iiint_{\mathbb{R}^{4}}\left\{\operatorname{div}\left(I_{W}^{0}, I_{W}^{1}, I_{W}^{2}, I_{W}^{3}\right)\right\}_{K} \xi\left(x^{0}, x^{1}, x^{2}, x^{3}\right)\left|\operatorname{det}\left(\left\{K^{m n}\right\}_{n, m=0,1,2,3}\right)\right|^{-\frac{1}{2}} d x^{0} d x^{1} d x^{2} d x^{3}= \\
& \iiint_{\mathbb{R}^{4}} \int \sum_{j=0}^{3} \frac{\partial I_{W}^{j}}{\partial x^{j}}\left(x^{0}, x^{1}, x^{2}, x^{3}\right) \xi\left(x^{0}, x^{1}, x^{2}, x^{3}\right)\left|\operatorname{det}\left(\left\{K^{m n}\right\}_{n, m=0,1,2,3}\right)\right|^{-\frac{1}{2}} d x^{0} d x^{1} d x^{2} d x^{3}+ \\
& \iiint_{\mathbb{R}^{4}} \int \sum_{j=0}^{3} I_{W}^{j}\left(x^{0}, x^{1}, x^{2}, x^{3}\right) \xi\left(x^{0}, x^{1}, x^{2}, x^{3}\right) \frac{\partial}{\partial x^{j}}\left(\left|\operatorname{det}\left(\left\{K^{m n}\right\}_{n, m=0,1,2,3}\right)\right|^{-\frac{1}{2}}\right) d x^{0} d x^{1} d x^{2} d x^{3} .
\end{aligned}
$$


However, by the definition of the derivative of the distribution we have,

$$
\begin{aligned}
& \iiint_{\mathbb{R}^{4}} \int_{j=0} \sum_{j=0} \frac{\partial I_{W}^{j}}{\partial x^{j}}\left(x^{0}, x^{1}, x^{2}, x^{3}\right)\left(\left|\operatorname{det}\left(\left\{K^{m n}\right\}_{n, m=0,1,2,3}\right)\right|^{-\frac{1}{2}} \xi\right)\left(x^{0}, x^{1}, x^{2}, x^{3}\right) d x^{0} d x^{1} d x^{2} d x^{3}= \\
& -\iiint \int_{\mathbb{R}^{4}} \sum_{j=0}^{3} I_{W}^{j} \frac{\partial}{\partial x^{j}}\left(\left|\operatorname{det}\left(\left\{K^{m n}\right\}_{n, m=0,1,2,3}\right)\right|^{-\frac{1}{2}} \xi\right)\left(x^{0}, x^{1}, x^{2}, x^{3}\right) d x^{0} d x^{1} d x^{2} d x^{3}= \\
& \quad-\iiint \int_{\mathbb{R}^{4}} \sum_{j=0}^{3} I_{W}^{j} \frac{\partial}{\partial x^{j}}\left(\left|\operatorname{det}\left(\left\{K^{m n}\right\}_{n, m=0,1,2,3}\right)\right|^{-\frac{1}{2}}\right) \xi\left(x^{0}, x^{1}, x^{2}, x^{3}\right) d x^{0} d x^{1} d x^{2} d x^{3} \\
& -\iiint \int_{\mathbb{R}^{4}}^{3} \sum_{j=0}^{3} I_{W}^{j}\left|\operatorname{det}\left(\left\{K^{m n}\right\}_{n, m=0,1,2,3}\right)\right|^{-\frac{1}{2}} \frac{\partial \xi}{\partial x^{j}}\left(x^{0}, x^{1}, x^{2}, x^{3}\right) d x^{0} d x^{1} d x^{2} d x^{3} . \quad \text { (10.194) }
\end{aligned}
$$

Thus, inserting (10.194) into (10.193) gives

$$
\begin{array}{r}
\iiint \int_{\mathbb{R}^{4}}\left\{\operatorname{div}\left(I_{W}^{0}, I_{W}^{1}, I_{W}^{2}, I_{W}^{3}\right)\right\}_{K} \xi\left(x^{0}, x^{1}, x^{2}, x^{3}\right)\left|\operatorname{det}\left(\left\{K^{m n}\right\}_{n, m=0,1,2,3}\right)\right|^{-\frac{1}{2}} d x^{0} d x^{1} d x^{2} d x^{3}= \\
-\iiint \int_{\mathbb{R}^{4}} \sum_{j=0}^{3} I_{W}^{j}\left|\operatorname{det}\left(\left\{K^{m n}\right\}_{n, m=0,1,2,3}\right)\right|^{-\frac{1}{2}} \frac{\partial \xi}{\partial x^{j}}\left(x^{0}, x^{1}, x^{2}, x^{3}\right) d x^{0} d x^{1} d x^{2} d x^{3} \cdot \quad
\end{array}
$$

On the other hand, by (3.5) we deduce:

$$
\begin{array}{r}
\iiint \int_{\mathbb{R}^{4}} \sum_{j=0}^{3} I_{W}^{j}\left(x^{0}, x^{1}, x^{2}, x^{3}\right)\left(\left|\operatorname{det}\left(\left\{K^{m n}\right\}_{n, m=0,1,2,3}\right)\right|^{-\frac{1}{2}} \frac{\partial \xi}{\partial x^{j}}\right)\left(x^{0}, x^{1}, x^{2}, x^{3}\right) d x^{0} d x^{1} d x^{2} d x^{3} \\
=\int_{a}^{b} W(s)\left(\sum_{j=0}^{3} \frac{\partial \xi}{\partial x^{j}}(\chi(s)) \frac{d \chi^{j}}{d s}(s)\right) d s=\int_{a}^{b} W(s) \frac{d}{d s}(\xi(\chi(s))) d s . \quad \text { (10.196) }
\end{array}
$$

Thus, if we assume that $W(s)$ is a constant across the trajectory of the motion, so that $W(s)=W_{0}$ where $W_{0}$ is independent on $s$, then by (10.195) and (10.196), using the Newton-Leibnitz formula, we deduce:

$$
\begin{gathered}
\iiint \int_{\mathbb{R}^{4}}\left\{\operatorname{div}\left(I_{W}^{0}, I_{W}^{1}, I_{W}^{2}, I_{W}^{3}\right)\right\}_{K} \xi\left(x^{0}, x^{1}, x^{2}, x^{3}\right)\left|\operatorname{det}\left(\left\{K^{m n}\right\}_{n, m=0,1,2,3}\right)\right|^{-\frac{1}{2}} d x^{0} d x^{1} d x^{2} d x^{3} \\
=-\int_{a}^{b} W(s) \frac{d}{d s}(\xi(\chi(s))) d s=-W_{0} \int_{a}^{b} \frac{d}{d s}(\xi(\chi(s))) d s=W_{0}(\xi(\chi(a))-\xi(\chi(b))) .
\end{gathered}
$$

However, since $\xi\left(x^{0}, x^{1}, x^{2}, x^{3}\right) \in C_{c}^{\infty}\left(\mathbb{R}^{4}\right)$ has a compact support, by (3.8) we obtain

$$
\xi(\chi(b))=\xi(\chi(a))=0,
$$

and thus, inserting (10.198) into (10.197) gives:

$$
\begin{array}{r}
\iiint \int_{\mathbb{R}^{4}}\left\{\operatorname{div}\left(I_{W}^{0}, I_{W}^{1}, I_{W}^{2}, I_{W}^{3}\right)\right\}_{K} \xi\left(x^{0}, x^{1}, x^{2}, x^{3}\right)\left|\operatorname{det}\left(\left\{K^{m n}\right\}_{n, m=0,1,2,3}\right)\right|^{-\frac{1}{2}} d x^{0} d x^{1} d x^{2} d x^{3} \\
=0 \quad \forall \xi \in C_{c}^{\infty}\left(\mathbb{R}^{4}\right) .
\end{array}
$$


Therefore, since the test function $\xi\left(x^{0}, x^{1}, x^{2}, x^{3}\right) \in C_{c}^{\infty}\left(\mathbb{R}^{4}\right)$ in (10.199) is arbitrary, by (10.199), using the basic properties of distributions, we finally deduce (3.9). This completes the proof.

Proof of Lemma 4.1. By inserting (4.60) into (4.58) we deduce:

$$
\left\{\begin{array}{l}
F_{00}=0 \\
F_{0 j}=-F_{j 0}=-\frac{\partial\left(-A_{j}\right)}{\partial x^{0}}-\frac{\partial \Psi}{\partial x^{j}} \quad \forall j=1,2,3 \\
F_{j j}=0 \quad \forall j=1,2,3 \\
F_{m j}=-F_{j m}=\frac{\partial\left(-A_{m}\right)}{\partial x^{j}}-\frac{\partial\left(-A_{j}\right)}{\partial x^{m}} \quad \forall m \neq j=1,2,3,
\end{array}\right.
$$

Thus, if we define, as usual the magnetic and the electric field:

$$
\left\{\begin{array}{l}
\mathbf{B}:=\operatorname{curl}_{\mathbf{x}} \mathbf{A}, \\
\mathbf{E}:=-\nabla_{\mathbf{x}} \Psi-\frac{\partial \mathbf{A}}{\partial x^{0}},
\end{array}\right.
$$

then denoting $\mathbf{E}:=\left(E_{1}, E_{2}, E_{3}\right)$ and $\mathbf{B}:=\left(B_{1}, B_{2}, B_{3}\right)$, by (10.201) we rewrite (10.200) as:

$$
\left\{\begin{array}{l}
F_{00}=0 \\
F_{0 j}=-F_{j 0}=E_{j} \quad \forall j=1,2,3 \\
F_{j j}=0 \quad \forall j=1,2,3 \\
F_{12}=-F_{21}=-B_{3} \\
F_{13}=-F_{31}=B_{2} \\
F_{23}=-F_{32}=-B_{1}
\end{array}\right.
$$

so that we get (4.63). Next consider two-times contravariant tensor $\left\{F^{m n}\right\}_{m, n=0,1,2,3}$ defined by:

$$
F^{m n}:=\sum_{k=0}^{3} \sum_{j=0}^{3} K^{m j} K^{n k} F_{j k} \quad \forall m, n=0,1,2,3 .
$$

We rewrite (10.203) as:

$$
\begin{aligned}
& F^{m n}= \\
& K^{m 0} K^{n 0} F_{00}+\sum_{k=1}^{3} K^{m 0} K^{n k} F_{0 k}+\sum_{j=1}^{3} K^{m j} K^{n 0} F_{j 0}+\sum_{k=1}^{3} \sum_{j=1}^{3} K^{m j} K^{n k} F_{j k} \quad \forall m, n=0,1,2,3 .
\end{aligned}
$$

In particular, by inserting (4.29) into (10.204) we deduce:

$$
\left\{\begin{array}{l}
F^{00}=F_{00}+\sum_{k=1}^{3} v^{k} F_{0 k}+\sum_{j=1}^{3} v^{j} F_{j 0}+\sum_{k=1}^{3} \sum_{j=1}^{3} v^{j} v^{k} F_{j k} \\
F^{m 0}=v^{m} F^{00}-F_{m 0}-\sum_{k=1}^{3} v^{k} F_{m k} \quad \forall m=1,2,3, \\
F^{0 n}=v^{n} F^{00}-F_{0 n}-\sum_{j=1}^{3} v^{j} F_{j n} \quad \forall n=1,2,3, \\
F^{m n}=v^{m} v^{n} F^{00}-\sum_{k=1}^{3} v^{n} v^{k} F_{m k}-\sum_{j=1}^{3} v_{m} v^{j} F_{j n}-v^{m} F_{0 n}-v^{n} F_{m 0}+F_{m n} \quad \forall m, n=1,2,3 .
\end{array}\right.
$$


We rewrite (10.205) as:

$$
\left\{\begin{array}{l}
F^{00}=F_{00}+\sum_{k=1}^{3} v^{k} F_{0 k}+\sum_{j=1}^{3} v^{j} F_{j 0}+\sum_{k=1}^{3} \sum_{j=1}^{3} v^{j} v^{k} F_{j k} \\
F^{m 0}=v^{m} F^{00}-F_{m 0}-\sum_{k=1}^{3} v^{k} F_{m k} \quad \forall m=1,2,3, \\
F^{0 n}=v^{n} F^{00}-F_{0 n}-\sum_{j=1}^{3} v^{j} F_{j n} \quad \forall n=1,2,3, \\
F^{m n}=v^{m} F^{0 n}+v^{n} F^{m 0}-v^{m} v^{n} F^{00}+F_{m n} \quad \forall m, n=1,2,3 .
\end{array}\right.
$$

In particular, since the tensor $\left\{F_{m n}\right\}_{m, n=0,1,2,3}$ is antisymmetric, i.e. $F_{m n}=-F_{n m} \forall m, n=$ $0,1,2,3$, then we simplify (10.206) as

$$
\left\{\begin{array}{l}
F^{00}=0 \\
F^{m m}=0 \quad \forall m=1,2,3, \\
F^{0 m}=-F^{m 0}=-F_{0 m}+\sum_{k=1}^{3} v^{k} F_{m k} \quad \forall m=1,2,3, \\
F^{m n}=v^{m} F^{0 n}-v^{n} F^{0 m}+F_{m n} \quad \forall m, n=1,2,3 .
\end{array}\right.
$$

In particular, since $\left\{F_{m j}\right\}_{0 \leq m, j \leq 3}$ is the antisymmetric two times covariant tensor field, then by inserting (10.202) into (10.207) we deduce:

$$
\left\{\begin{array}{l}
F^{00}=0 \\
F^{j j}=0 \quad \forall j=1,2,3 \\
F^{01}=-F^{10}=-F_{01}+v^{2} F_{12}+v^{3} F_{13}=-\left(E_{1}+\left(v^{2} B_{3}-v^{3} B_{2}\right)\right) \\
F^{02}=-F^{20}=-F_{02}+v^{1} F_{21}+v^{3} F_{23}=-\left(E_{2}+\left(v^{3} B_{1}-v^{1} B_{3}\right)\right) \\
F^{03}=-F^{30}=-F_{03}+v^{1} F_{31}+v^{2} F_{32}=-\left(E_{3}+\left(v^{1} B_{2}-v^{2} B_{1}\right)\right) \\
F^{12}=-F^{21}=v^{1} F^{02}-v^{2} F^{01}+F_{12}=-\left(B_{3}+\left(v^{1} F^{20}-v^{2} F^{10}\right)\right) \\
F^{13}=-F^{31}=v^{1} F^{03}-v^{3} F^{01}+F_{13}=B_{2}+\left(v^{3} F^{10}-v^{1} F^{30}\right) \\
F^{23}=-F^{32}=v^{2} F^{03}-v^{3} F^{02}+F_{23}=-\left(B_{1}+\left(v^{2} F^{30}-v^{3} F^{20}\right)\right) .
\end{array} .\right.
$$

Thus, defining:

$$
\left\{\begin{array}{l}
\mathbf{D}:=\mathbf{E}+\mathbf{v} \times \mathbf{B} \\
\mathbf{H}:=\mathbf{B}+\mathbf{v} \times \mathbf{D},
\end{array}\right.
$$

and denoting $\mathbf{D}:=\left(D_{1}, D_{2}, D_{3}\right)$ and $\mathbf{H}:=\left(H_{1}, H_{2}, H_{3}\right)$ we rewrite (10.208) as:

$$
\left\{\begin{array}{l}
F^{00}=0 \\
F^{0 j}=-F^{j 0}=-D_{j} \quad \forall j=1,2,3, \\
F^{j j}=0 \quad \forall j=1,2,3 \\
F^{12}=-F^{21}=-H_{3} \\
F^{13}=-F^{31}=H_{2} \\
F^{23}=-F^{32}=-H_{1}
\end{array}\right.
$$


so that we get (4.64). In particular, by (10.202) and (10.210), using (10.209) and using the definition (4.58), we deduce that

$$
\begin{aligned}
\left(\sum_{n=0}^{3} \sum_{k=0}^{3} \sum_{m=0}^{3} \sum_{p=0}^{3} K^{m n} K^{p k}\left(\frac{\partial A_{p}}{\partial x^{m}}-\frac{\partial A_{m}}{\partial x^{p}}\right)\left(\frac{\partial A_{k}}{\partial x^{n}}-\frac{\partial A_{n}}{\partial x^{k}}\right)\right)=\sum_{j=0}^{3} \sum_{k=0}^{3} F^{j k} F_{j k} \\
=F^{00} F_{00}+\sum_{k=1}^{3} F^{0 k} F_{0 k}+\sum_{j=1}^{3} F^{j 0} F_{j 0}+\sum_{j=1}^{3} \sum_{k=1}^{3} F^{j k} F_{j k}=-2 \mathbf{E} \cdot \mathbf{D}+2 \mathbf{B} \cdot \mathbf{H}= \\
-2((\mathbf{D}-\mathbf{v} \times \mathbf{B}) \cdot \mathbf{D}-\mathbf{B} \cdot(\mathbf{B}+\mathbf{v} \times \mathbf{D}))=-2\left(|\mathbf{D}|^{2}-|\mathbf{B}|^{2}\right),
\end{aligned}
$$

and so by (10.211), (10.209) and (10.201) we finally obtain (4.65).

Proof of Lemma 6.1. Since the current coordinate system is cartesian, by Theorem 2.1 there exists a change of variables from the kinematically preferable coordinate system to the current cartesian coordinate system of the form:

$$
\left\{\begin{array}{l}
x^{0}=x^{\prime 0}+c, \\
x^{m}=\sum_{j=1}^{3} A_{m j}\left(x^{\prime 0}\right) x^{\prime j}+z^{m}\left(x^{\prime 0}\right) \quad \forall m=1,2,3,
\end{array}\right.
$$

where $\left\{A_{m n}\left(x^{\prime 0}\right)\right\}_{n, m=1,2,3} \in \mathbb{R}^{3 \times 3}$ is a $3 \times 3$-matrix, depending on the coordinate $x^{\prime 0}$ only (independent on $\left.\mathbf{x}^{\prime}:=\left(x^{\prime 1}, x^{\prime 2}, x^{\prime 3}\right)\right)$, and satisfying

$\sum_{j=1}^{3} A_{m j}\left(x^{\prime 0}\right) A_{n j}\left(x^{\prime 0}\right)=\sum_{j=1}^{3} A_{j m}\left(x^{\prime 0}\right) A_{j n}\left(x^{\prime 0}\right)=\delta_{m n} \quad \forall m, n=1,2,3 \quad \forall\left(x^{\prime 0}, x^{\prime 1}, x^{\prime 2}, x^{\prime 3}\right) \in \mathbb{R}^{4}$,

$c \in \mathbb{R}$ is a constant (independent on $\left.\left(x^{\prime 0}, x^{\prime 1}, x^{\prime 2}, x^{\prime 3}\right) \in \mathbb{R}^{4}\right)$ and $\mathbf{z}\left(x^{\prime 0}\right):=\left(z^{1}\left(x^{\prime 0}\right), z^{2}\left(x^{\prime 0}\right), z^{3}\left(x^{\prime 0}\right)\right) \in$ $\mathbb{R}^{3}$ is a three-dimensional vector field, depending on the coordinate $x^{\prime 0}$ only (independent on $\left.\mathbf{x}^{\prime}:=\left(x^{\prime 1}, x^{\prime 2}, x^{\prime 3}\right)\right)$. On the other hand, since the kinematically preferable system is simultaneously Lorentzian and cartesian, in this system we have

$$
\left(r^{\prime 0}, r^{\prime 1}, r^{\prime 2}, r^{\prime 3}\right)=(1,0,0,0) \quad \text { so that } \quad \mathbf{r}^{\prime}:=\left(r^{\prime 1}, r^{\prime 2}, r^{\prime 3}\right)=(0,0,0) .
$$

On the other hand, by the rule of transformations of contravariant vector in (10.3), using (10.212), (10.214) and (10.213) we deduce

$$
r^{0}=1,
$$

and

$$
\begin{aligned}
r^{m}=\sum_{j=1}^{3} \frac{d A_{m j}}{d x^{\prime 0}}\left(x^{\prime 0}\right) x^{\prime j}+\frac{d z^{m}}{d x^{\prime 0}}\left(x^{\prime 0}\right)= \\
\sum_{j=1}^{3} \sum_{n=1}^{3} \frac{d A_{m j}}{d x^{0}}\left(x^{0}\right) A_{n j}\left(x^{0}\right)\left(x^{n}-z^{n}\left(x^{0}\right)\right)+\frac{d z^{m}}{d x^{0}}\left(x^{0}\right) \quad \forall m=1,2,3 .
\end{aligned}
$$


Therefore, differentiating (10.216) gives:

$$
\frac{\partial r^{m}}{\partial x^{n}}=\sum_{j=1}^{3} \frac{d A_{m j}}{d x^{0}}\left(x^{0}\right) A_{n j}\left(x^{0}\right) \quad \forall m, n=1,2,3 .
$$

Thus, by (10.217) and (10.213) we deduce

$$
\begin{aligned}
\left(\frac{\partial r^{m}}{\partial x^{n}}+\frac{\partial r^{n}}{\partial x^{m}}\right) & =\sum_{j=1}^{3}\left(\frac{d A_{m j}}{d x^{0}}\left(x^{0}\right) A_{n j}\left(x^{0}\right)+\frac{d A_{n j}}{d x^{0}}\left(x^{0}\right) A_{m j}\left(x^{0}\right)\right) \\
& =\frac{d}{d x^{0}}\left\{\sum_{j=1}^{3} A_{m j}\left(x^{0}\right) A_{n j}\left(x^{0}\right)\right\}=\frac{d}{d x^{0}}\left\{\delta_{m n}\right\}=0 \quad \forall m, n=1,2,3 .
\end{aligned}
$$

In particular,

$$
\left\{\begin{array}{l}
\left(\frac{\partial r^{m}}{\partial x^{n}}+\frac{\partial r^{n}}{\partial x^{m}}\right)=0 \quad \forall m, n=1,2,3 \\
\operatorname{div}_{\mathbf{x}} \mathbf{r}=\sum_{n=1}^{3} \frac{\partial r^{n}}{\partial x^{n}}=0
\end{array}\right.
$$

Moreover, differentiating (10.217) one more time by $x^{j}$ gives

$$
\frac{\partial^{2} r^{m}}{\partial x^{j} \partial x^{n}}=0 \quad \forall j, m, n=1,2,3 .
$$

So, by (10.219) and (10.220) we deduce (6.3).

Proof of Lemma 9.1. By the Theory of Distribution, the definition in (9.4) means that for every smooth scalar classical function with compact support $\xi\left(x^{0}, x^{1}, x^{2}, x^{3}\right) \in C_{c}^{\infty}\left(\mathbb{R}^{4}\right)$ we have

$$
\begin{array}{r}
\iiint \int_{\mathbb{R}^{4}}\left|\operatorname{det}\left(\left\{K^{p q}\right\}_{p, q=0,1,2,3}\right)\right|^{-\frac{1}{2}} \Upsilon_{\sigma}^{m n}\left(x^{0}, x^{1}, x^{2}, x^{3}\right), \xi\left(x^{0}, x^{1}, x^{2}, x^{3}\right) d x^{0} d x^{1} d x^{2} d x^{3}=\int_{0}^{1} \int_{a}^{b} \sigma\{ \\
\left(\chi^{m}(s)-z^{m}(s)\right)\left(\tau \frac{d \chi^{n}}{d s}(s)+(1-\tau) \frac{d z^{n}}{d s}(s)\right)-\left(\chi^{n}(s)-z^{n}(s)\right)\left(\tau \frac{d \chi^{m}}{d s}(s)+(1-\tau) \frac{d z^{m}}{d s}(s)\right) \\
\} \xi(\tau \chi(s)+(1-\tau) z(s)) d s d \tau \quad \forall m=0,1,2,3 . \quad(10.221)
\end{array}
$$

Next, since by the Theory of Distributions we have

$$
\begin{aligned}
& \sum_{n=0}^{3} \iiint_{\mathbb{R}^{4}} \int \frac{\partial}{\partial x^{n}}\left\{\left|\operatorname{det}\left(\left\{K^{p q}\right\}_{p, q=0,1,2,3}\right)\right|^{-\frac{1}{2}} \Upsilon_{\sigma}^{m n}\right\} \xi\left(x^{0}, x^{1}, x^{2}, x^{3}\right) d x^{0} d x^{1} d x^{2} d x^{3}= \\
&-\sum_{n=0}^{3} \iiint_{\mathbb{R}^{4}} \int\left|\operatorname{det}\left(\left\{K^{p q}\right\}_{p, q=0,1,2,3}\right)\right|^{-\frac{1}{2}} \Upsilon_{\sigma}^{m n} \frac{\partial \xi}{\partial x^{n}}\left(x^{0}, x^{1}, x^{2}, x^{3}\right) d x^{0} d x^{1} d x^{2} d x^{3} \quad \forall m=0,1,2,3,
\end{aligned}
$$


by $(10.221)$ we can proceed in (10.222) as:

$$
\begin{array}{r}
\sum_{n=0}^{3} \iiint_{\mathbb{R}^{4}} \frac{\partial}{\partial x^{n}}\left\{\left|\operatorname{det}\left(\left\{K^{p q}\right\}_{p, q=0,1,2,3}\right)\right|^{-\frac{1}{2}} \Upsilon_{\sigma}^{m n}\right\} \xi\left(x^{0}, x^{1}, x^{2}, x^{3}\right) d x^{0} d x^{1} d x^{2} d x^{3}= \\
-\sum_{n=0}^{3} \int_{0}^{1} \int_{a}^{b} \sigma\left\{\left(\chi^{m}(s)-z^{m}(s)\right)\left(\tau \frac{d \chi^{n}}{d s}(s)+(1-\tau) \frac{d z^{n}}{d s}(s)\right)\right. \\
\left.-\left(\chi^{n}(s)-z^{n}(s)\right)\left(\tau \frac{d \chi^{m}}{d s}(s)+(1-\tau) \frac{d z^{m}}{d s}(s)\right)\right\} \frac{\partial \xi}{\partial x^{n}}(\tau \chi(s)+(1-\tau) z(s)) d s d \tau \\
\forall m=0,1,2,3 .
\end{array}
$$

Furthermore, by the Chain Rule we can rewrite (10.223) as:

$$
\begin{aligned}
& \sum_{n=0}^{3} \iiint_{\mathbb{R}^{4}} \int_{0} \frac{\partial}{\partial x^{n}}\left\{\left|\operatorname{det}\left(\left\{K^{p q}\right\}_{p, q=0,1,2,3}\right)\right|^{-\frac{1}{2}} \Upsilon_{\sigma}^{m n}\right\} \xi\left(x^{0}, x^{1}, x^{2}, x^{3}\right) d x^{0} d x^{1} d x^{2} d x^{3}= \\
& \quad-\int_{0}^{1} \int_{a}^{b} \sigma\left(\chi^{m}(s)-z^{m}(s)\right) \frac{\partial \xi}{\partial s}(\tau \chi(s)+(1-\tau) z(s)) d s d \tau \\
& +\int_{0}^{1} \int_{a}^{b} \sigma\left(\tau \frac{d \chi^{m}}{d s}(s)+(1-\tau) \frac{d z^{m}}{d s}(s)\right) \frac{\partial \xi}{\partial \tau}(\tau \chi(s)+(1-\tau) z(s)) d s d \tau \quad \forall m=0,1,2,3 .
\end{aligned}
$$

However, taking into account (9.3), integration by partes by $s$ gives:

$$
\begin{aligned}
-\int_{0}^{1} \int_{a}^{b} \sigma & \left(\chi^{m}(s)-z^{m}(s)\right) \frac{\partial \xi}{\partial s}(\tau \chi(s)+(1-\tau) z(s)) d s d \tau= \\
& \int_{0}^{1} \int_{a}^{b} \sigma\left(\frac{d \chi^{m}}{d s}(s)-\frac{d z^{m}}{d s}(s)\right) \xi(\tau \chi(s)+(1-\tau) z(s)) d s d \tau \quad \forall m=0,1,2,3 .
\end{aligned}
$$

On the other hand, integration by partes by $\tau$ gives:

$$
\begin{aligned}
\int_{0}^{1} \int_{a}^{b} \sigma\left(\tau \frac{d \chi^{m}}{d s}(s)+\right. & \left.(1-\tau) \frac{d z^{m}}{d s}(s)\right) \frac{\partial \xi}{\partial \tau}(\tau \chi(s)+(1-\tau) z(s)) d s d \tau \\
& \left.\left.+\int_{a}^{b} \sigma \frac{d \chi^{m}}{d s}(s) \xi(\chi(s))\right) d s-\int_{a}^{b} \sigma \frac{d z^{m}}{d s}(s) \xi(z(s))\right) d s \\
& -\int_{0}^{1} \int_{a}^{b} \sigma\left(\frac{d \chi^{m}}{d s}(s)-\frac{d z^{m}}{d s}(s)\right) \xi(\tau \chi(s)+(1-\tau) z(s)) d s d \tau \quad \forall m=0,1,2,3 .
\end{aligned}
$$


Thus, inserting (10.225) and (10.226) into (10.224) gives:

$$
\begin{array}{r}
\sum_{n=0}^{3} \iiint_{\mathbb{R}^{4}} \int \frac{\partial}{\partial x^{n}}\left\{\left|\operatorname{det}\left(\left\{K^{p q}\right\}_{p, q=0,1,2,3}\right)\right|^{-\frac{1}{2}} \Upsilon_{\sigma}^{m n}\right\} \xi\left(x^{0}, x^{1}, x^{2}, x^{3}\right) d x^{0} d x^{1} d x^{2} d x^{3}= \\
\left.\left.\int_{a}^{b} \sigma \frac{d \chi^{m}}{d s}(s) \xi(\chi(s))\right) d s-\int_{a}^{b} \sigma \frac{d z^{m}}{d s}(s) \xi(z(s))\right) d s \quad \forall m=0,1,2,3 .
\end{array}
$$

Thus, since $\xi\left(x^{0}, x^{1}, x^{2}, x^{3}\right) \in C_{c}^{\infty}\left(\mathbb{R}^{4}\right)$ was chosen arbitrary, by the Theory of Distributions, using (10.227) we deduce

$$
\begin{aligned}
& \sum_{n=0}^{3} \frac{\partial}{\partial x^{n}}\left\{\left|\operatorname{det}\left(\left\{K^{p q}\right\}_{p, q=0,1,2,3}\right)\right|^{-\frac{1}{2}} \Upsilon_{\sigma}^{m n}\right\}= \\
& \int_{a}^{b} \sigma \frac{d \chi^{m}}{d s}(s) \delta\left(x^{0}-\chi^{0}(s), \ldots, x^{3}-\chi^{3}(s)\right) d s-\int_{a}^{b} \sigma \frac{d z^{m}}{d s}(s) \delta\left(x^{0}-z^{0}(s), \ldots, x^{3}-z^{3}(s)\right) d s .
\end{aligned}
$$

Finally, (10.228) obviously implies:

$$
\begin{aligned}
& \sum_{n=0}^{3} \frac{1}{\left.\operatorname{det}\left(\left\{K^{p q}\right\}_{p, q=0,1,2,3}\right)\right|^{-\frac{1}{2}} \frac{\partial}{\partial x^{n}}\left\{\left|\operatorname{det}\left(\left\{K^{p q}\right\}_{(p, q)_{0}^{3}}\right)\right|^{-\frac{1}{2}} \Upsilon_{\sigma}^{m n}\right\}=} \\
& \left|\operatorname{det}\left(\left\{K^{p q}\right\}_{p, q=0,1,2,3}\right)\right|^{\frac{1}{2}} \int_{a}^{b} \sigma \frac{d \chi^{m}}{d s}(s) \delta\left(x^{0}-\chi^{0}(s), \ldots, x^{3}-\chi^{3}(s)\right) d s \\
& -\left|\operatorname{det}\left(\left\{K^{p q}\right\}_{p, q=0,1,2,3}\right)\right|^{\frac{1}{2}} \int_{a}^{b} \sigma \frac{d z^{m}}{d s}(s) \delta\left(x^{0}-z^{0}(s), \ldots, x^{3}-z^{3}(s)\right) d s .
\end{aligned}
$$

\section{Appendix: some technical statements}

Lemma 11.1. Assume that a contravariant pseudo-metric $\left\{K^{m n}\right\}_{m, n=0,1,2,3}$ and a covector of the time-direction $\left(w_{0}, w_{1}, w_{2}, w_{3}\right)$ are weakly correlated (see Definition 2.3), so that we have everywhere

$$
\sum_{j=0}^{3} \sum_{m=0}^{3} K^{j m} w_{j} w_{m}>0 \quad \forall\left(x^{0}, x^{1}, x^{2}, x^{3}\right) \in \mathbb{R}^{4} .
$$

Moreover, assume that there exists a scalar field $\varphi$ such that

$$
\begin{gathered}
\lim _{\left\{\left(x^{0}\right)^{2}+\left(x^{1}\right)^{2}+\left(x^{2}\right)^{2}+\left(x^{3}\right)^{2}\right\} \rightarrow+\infty}\left(\sum_{j=0}^{3} \sum_{m=0}^{3} K^{j m} \frac{\partial \varphi}{\partial x^{j}} \frac{\partial \varphi}{\partial x^{m}}\right)=1, \\
\left(w_{0}, w_{1}, w_{2}, w_{3}\right)=\left(\frac{\partial \varphi}{\partial x^{0}}, \frac{\partial \varphi}{\partial x^{1}}, \frac{\partial \varphi}{\partial x^{2}}, \frac{\partial \varphi}{\partial x^{3}}\right) \quad \forall\left(x^{0}, x^{1}, x^{2}, x^{3}\right) \in \mathbb{R}^{4},
\end{gathered}
$$


and

$$
\sum_{j=0}^{3} \frac{\partial}{\partial x^{j}}\left\{\left(\sum_{m=0}^{3} \sum_{n=0}^{3} K^{m n} \frac{\partial \varphi}{\partial x^{m}} \frac{\partial \varphi}{\partial x^{n}}-1\right)\left(\sum_{n=0}^{3} K^{j n} \frac{\partial \varphi}{\partial x^{n}}\right)\right\}=0 \quad \forall\left(x^{0}, x^{1}, x^{2}, x^{3}\right) \in \mathbb{R}^{4} .
$$

Then, scalar global time $\varphi$ and pseudo-metric $\left\{K^{m n}\right\}_{m, n=0,1,2,3}$ are strongly correlated on $\mathbb{R}^{4}$, i.e. $\varphi$ satisfies the following eikonal-type equation:

$$
\sum_{j=0}^{3} \sum_{m=0}^{3} K^{j m} \frac{\partial \varphi}{\partial x^{j}} \frac{\partial \varphi}{\partial x^{m}}=1 \quad \forall\left(x^{0}, x^{1}, x^{2}, x^{3}\right) \in \mathbb{R}^{4} .
$$

Proof. Define

$$
\begin{aligned}
& \mathcal{A}_{1}=\left\{\left(x^{0}, x^{1}, x^{2}, x^{3}\right) \in \mathbb{R}^{4}:\left(\sum_{j=0}^{3} \sum_{m=0}^{3} K^{j m} \frac{\partial \varphi}{\partial x^{j}} \frac{\partial \varphi}{\partial x^{m}}\right)\left(x^{0}, x^{1}, x^{2}, x^{3}\right)-1<0\right\}, \\
& \mathcal{A}_{2}=\left\{\left(x^{0}, x^{1}, x^{2}, x^{3}\right) \in \mathbb{R}^{4}:\left(\sum_{j=0}^{3} \sum_{m=0}^{3} K^{j m} \frac{\partial \varphi}{\partial x^{j}} \frac{\partial \varphi}{\partial x^{m}}\right)\left(x^{0}, x^{1}, x^{2}, x^{3}\right)-1>0\right\} .
\end{aligned}
$$

Then, we obviously have

$$
\sum_{j=0}^{3} \sum_{m=0}^{3} K^{j m} \frac{\partial \varphi}{\partial x^{j}} \frac{\partial \varphi}{\partial x^{m}}-1=0 \quad \forall\left(x^{0}, x^{1}, x^{2}, x^{3}\right) \in \partial \mathcal{A}_{1} \cup \partial \mathcal{A}_{2} .
$$

where by $\partial \mathcal{A}$ we denote the boundary of the set $\mathcal{A}$. Therefore, by (11.4), (11.2) and (11.8), the Gauss-Green formula gives

$$
\begin{gathered}
\iiint \int_{\mathcal{A}_{k}}\left\{\left(\sum_{m=0}^{3} \sum_{n=0}^{3} K^{m n} \frac{\partial \varphi}{\partial x^{m}} \frac{\partial \varphi}{\partial x^{n}}-1\right)\left(\sum_{j=0}^{3} \sum_{n=0}^{3} K^{j n} \frac{\partial \varphi}{\partial x^{j}} \frac{\partial \varphi}{\partial x^{n}}\right)\right\} d\left(x^{0}, x^{1}, x^{2}, x^{3}\right)= \\
-\iiint \int_{\mathcal{A}_{k}} \varphi\left(\sum_{j=0}^{3} \frac{\partial}{\partial x^{j}}\left\{\left(\sum_{m=0}^{3} \sum_{n=0}^{3} K^{m n} \frac{\partial \varphi}{\partial x^{m}} \frac{\partial \varphi}{\partial x^{n}}-1\right)\left(\sum_{n=0}^{3} K^{j n} \frac{\partial \varphi}{\partial x^{n}}\right)\right\}\right) d\left(x^{0}, x^{1}, x^{2}, x^{3}\right) \\
=0 \quad \forall k=1,2 .
\end{gathered}
$$

However, by (11.1) and (11.3) we have

$$
\sum_{j=0}^{3} \sum_{m=0}^{3} K^{j m} \frac{\partial \varphi}{\partial x^{j}} \frac{\partial \varphi}{\partial x^{n}}>0 \quad \forall\left(x^{0}, x^{1}, x^{2}, x^{3}\right) \in \mathbb{R}^{4} .
$$

Thus, inserting (11.6), (11.7) and (11.10) into (11.9) gives

$$
\begin{aligned}
\iiint \int_{\mathcal{A}_{k}}\left|\left(\sum_{m=0}^{3} \sum_{n=0}^{3} K^{m n} \frac{\partial \varphi}{\partial x^{m}} \frac{\partial \varphi}{\partial x^{n}}-1\right)\left(\sum_{j=0}^{3} \sum_{n=0}^{3} K^{j n} \frac{\partial \varphi}{\partial x^{j}} \frac{\partial \varphi}{\partial x^{n}}\right)\right| d\left(x^{0}, x^{1}, x^{2}, x^{3}\right) \\
=0 \quad \forall k=1,2 .
\end{aligned}
$$

So, by the definition of $\mathcal{A}_{1}, \mathcal{A}_{2}$ we must have

$$
\iiint_{\mathbb{R}^{4}}\left|\left(\sum_{m=0}^{3} \sum_{n=0}^{3} K^{m n} \frac{\partial \varphi}{\partial x^{m}} \frac{\partial \varphi}{\partial x^{n}}-1\right)\left(\sum_{j=0}^{3} \sum_{n=0}^{3} K^{j n} \frac{\partial \varphi}{\partial x^{j}} \frac{\partial \varphi}{\partial x^{n}}\right)\right| d\left(x^{0}, x^{1}, x^{2}, x^{3}\right)=0,
$$

which, together with (11.10), finally implies (11.5). 
Lemma 11.2. Consider a contravariant pseudo-metric $\left\{K^{m n}\right\}_{m, n=0,1,2,3}$ on $\mathbb{R}^{4}$ and let $\left\{K_{m n}\right\}_{m, n=0,1,2,3}$ be the inverse covariant pseudo-metric on $\mathbb{R}^{4}$, associated with $\left\{K^{m n}\right\}_{m, n=0,1,2,3}$. Next, consider the Christoffel Symbols $\left\{\Gamma_{k j}^{m}\right\}_{K}$, defined by (2.25). Then, in some coordinate system we have

$$
\left\{\Gamma_{m n}^{j}\right\}_{K}=0 \quad \forall j, m, n=0,1,2,3,
$$

if and only if the tensor $\left\{K_{m n}\right\}_{m, n=0,1,2,3}$ is independent on the local coordinates $\left(x^{0}, x^{1}, x^{2}, x^{3}\right) \in \mathbb{R}^{4}$ in the given coordinate system.

Proof. If in some fixed coordinate system $\left\{K_{m n}\right\}_{m, n=0,1,2,3}$ is independent on the local coordinates $\left(x^{0}, x^{1}, x^{2}, x^{3}\right) \in \mathbb{R}^{4}$, then by (2.25) we obviously deduce (11.13).

Conversely, if in some fixed coordinate system we have (11.13), then we obviously have

$$
\frac{\partial K_{m n}}{\partial x^{j}}=\left\{\delta_{j} K_{m n}\right\}_{K}=0 \quad \forall j, m, n=0,1,2,3,
$$

where $\left\{\delta_{j} K_{m n}\right\}_{K}$ is the covariant derivative of the pseudo-metric $K$ with respect to the same pseudometric $K$, which is vanishes, due to the well known rule of the tensor analysis. So, by (11.14) we deduce that $\left\{K_{m n}\right\}_{m, n=0,1,2,3}$ is indeed independent on the local coordinates $\left(x^{0}, x^{1}, x^{2}, x^{3}\right) \in$ $\mathbb{R}^{4}$.

Lemma 11.3. Consider a contravariant pseudo-metric $\left\{K^{m n}\right\}_{m, n=0,1,2,3}$ on $\mathbb{R}^{4}$ and let $\left\{K_{m n}\right\}_{m, n=0,1,2,3}$ be the inverse covariant pseudo-metric on $\mathbb{R}^{4}$, associated with $\left\{K^{m n}\right\}_{m, n=0,1,2,3}$. Next, consider two coordinate systems in $\mathbb{R}^{4}$, so that the change of coordinates from the first to the second coordinate system is given by:

$$
\left\{\begin{array}{l}
x^{\prime 0}=f^{(0)}\left(x^{0}, x^{1}, x^{2}, x^{3}\right), \\
x^{\prime 1}=f^{(1)}\left(x^{0}, x^{1}, x^{2}, x^{3}\right), \\
x^{\prime 2}=f^{(2)}\left(x^{0}, x^{1}, x^{2}, x^{3}\right), \\
x^{\prime 3}=f^{(3)}\left(x^{0}, x^{1}, x^{2}, x^{3}\right) .
\end{array}\right.
$$

Finally, assume that the tensor $\left\{K_{m n}\right\}_{m, n=0,1,2,3}$ is independent on the local coordinates in both given coordinate systems, i.e. $\left\{K_{m n}\right\}_{m, n=0,1,2,3}$ is independent on the coordinates $\left(x^{0}, x^{1}, x^{2}, x^{3}\right) \in \mathbb{R}^{4}$ and $\left\{K_{m n}^{\prime}\right\}_{m, n=0,1,2,3}$ is independent on the coordinates $\left(x^{\prime 0}, x^{\prime 1}, x^{\prime 2}, x^{\prime 3}\right) \in \mathbb{R}^{4}$. Then, the transformations in (11.15) are linear, i.e.

$$
\frac{\partial^{2} f^{(j)}}{\partial x^{n} \partial x^{k}}\left(x^{0}, x^{1}, x^{2}, x^{3}\right)=0 \quad \forall\left(x^{0}, x^{1}, x^{2}, x^{3}\right) \in \mathbb{R}^{4} \quad \forall j, k, n=0,1,2,3 .
$$

Proof. First of all, observe that since $\left\{K_{m n}\right\}_{m, n=0,1,2,3}$ is independent on the coordinates $\left(x^{0}, x^{1}, x^{2}, x^{3}\right) \in \mathbb{R}^{4}$ and $\left\{K_{m n}^{\prime}\right\}_{m, n=0,1,2,3}$ is independent on the coordinates $\left(x^{\prime 0}, x^{\prime 1}, x^{\prime 2}, x^{\prime 3}\right) \in \mathbb{R}^{4}$, then, by (2.25) in both coordinate systems we have

$$
\left\{\Gamma_{m n}^{j}\right\}_{K}=0 \quad \forall j, m, n=0,1,2,3
$$


and

$$
\left\{\Gamma_{m n}^{\prime j}\right\}_{K^{\prime}}=0 \quad \forall j, m, n=0,1,2,3 .
$$

Next fix an index $j \in\{0,1,2,3\}$ and define the proper scalar field $\psi^{\prime}\left(x^{\prime 0}, x^{\prime 1}, x^{\prime 2}, x^{\prime 3}\right)=\psi\left(x^{0}, x^{1}, x^{2}, x^{3}\right)$ by the following

$$
\psi\left(x^{0}, x^{1}, x^{2}, x^{3}\right)=f^{(j)}\left(x^{0}, x^{1}, x^{2}, x^{3}\right) \quad \forall\left(x^{0}, x^{1}, x^{2}, x^{3}\right) \in \mathbb{R}^{4}
$$

so that we have

$$
\psi^{\prime}\left(x^{\prime 0}, x^{\prime 1}, x^{\prime 2}, x^{\prime 3}\right)=x^{\prime j} \quad \forall\left(x^{\prime 0}, x^{\prime 1}, x^{\prime 2}, x^{\prime 3}\right) \in \mathbb{R}^{4} .
$$

On the other hand, by (2.26) with the covector $\left(\frac{\partial \psi}{\partial x^{0}}, \frac{\partial \psi}{\partial x^{1}}, \frac{\partial \psi}{\partial x^{2}}, \frac{\partial \psi}{\partial x^{3}}\right)$ instead of $\left(h_{0}, h_{1}, h_{2}, h_{3}\right)$, using (11.17) and (11.18), in both coordinate systems we deduce

$$
\left\{\delta_{n}\left(\frac{\partial \psi}{\partial x^{k}}\right)\right\}_{K}=\frac{\partial^{2} \psi}{\partial x^{n} \partial x^{k}} \quad \forall n, k=0,1,2,3 \quad \forall\left(x^{0}, x^{1}, x^{2}, x^{3}\right) \in \mathbb{R}^{4},
$$

and

$$
\left\{\delta_{n}^{\prime}\left(\frac{\partial \psi^{\prime}}{\partial x_{k}^{\prime}}\right)\right\}_{K^{\prime}}=\frac{\partial^{2} \psi^{\prime}}{\partial x_{n}^{\prime} \partial x_{k}^{\prime}} \quad \forall n, k=0,1,2,3 \quad \forall\left(x^{\prime 0}, x^{\prime 1}, x^{\prime 2}, x^{\prime 3}\right) \in \mathbb{R}^{4} .
$$

However, by (11.20) and (11.22) together we deduce

$$
\left\{\delta_{n}^{\prime}\left(\frac{\partial \psi^{\prime}}{\partial x_{k}^{\prime}}\right)\right\}_{K^{\prime}}=\frac{\partial^{2} \psi^{\prime}}{\partial x_{n}^{\prime} \partial x_{k}^{\prime}}=\frac{\partial^{2} x^{\prime j}}{\partial x_{n}^{\prime} \partial x_{k}^{\prime}}=0 \quad \forall n, k=0,1,2,3 \quad \forall\left(x^{\prime 0}, x^{\prime 1}, x^{\prime 2}, x^{\prime 3}\right) \in \mathbb{R}^{4} .
$$

Therefore, since $\left\{\left\{\delta_{n}\left(\frac{\partial \psi}{\partial x^{k}}\right)\right\}_{K}\right\}_{n, k=0,1,2,3}$ is a proper two-times covariant tensor, by (11.23) we deduce

$$
\left\{\delta_{n}\left(\frac{\partial \psi}{\partial x^{k}}\right)\right\}_{K}=0 \quad \forall n, k=0,1,2,3 \quad \forall\left(x^{0}, x^{1}, x^{2}, x^{3}\right) \in \mathbb{R}^{4} .
$$

Thus, by (11.24) and (11.21) we deduce

$$
\frac{\partial^{2} \psi}{\partial x^{n} \partial x^{k}}=0 \quad \forall n, k=0,1,2,3 \quad \forall\left(x^{0}, x^{1}, x^{2}, x^{3}\right) \in \mathbb{R}^{4} .
$$

Finally, by (11.25) and (11.19) we obtain

$$
\frac{\partial^{2} f^{(j)}}{\partial x^{n} \partial x^{k}}\left(x^{0}, x^{1}, x^{2}, x^{3}\right)=0 \quad \forall\left(x^{0}, x^{1}, x^{2}, x^{3}\right) \in \mathbb{R}^{4} \quad \forall k, n=0,1,2,3,
$$

and since the fixed index $j \in\{0,1,2,3\}$ was arbitrary, we deduce (11.16).

Corollary 11.1. Let $\mathbf{f}(\mathbf{x}):=\mathbf{f}\left(x^{1}, x^{2}, x^{3}\right): \mathbb{R}^{3} \rightarrow \mathbb{R}^{3}$ be a smooth mapping. Next assume that the Jacoby's derivatives matrix $d_{\mathbf{x}} \mathbf{f}:=\left\{\frac{\partial f_{m}}{\partial x^{n}}\right\}_{1 \leq m, n \leq 3}$ of $\mathbf{f}$ satisfies (everywhere)

$$
\left\{d_{\mathbf{x}} \mathbf{f}(\mathbf{x})\right\}^{T} \cdot\left\{d_{\mathbf{x}} \mathbf{f}(\mathbf{x})\right\}=I \quad \forall \mathbf{x} \in \mathbb{R}^{3},
$$

where $I \in \mathbb{R}^{3 \times 3}$ is the identity matrix. Then $d_{\mathbf{x}} \mathbf{f}$ is a constant matrix (independent on $\mathbf{x}$ ) in $\mathbb{R}^{3}$ and so $\mathbf{f}$ is a linear orthogonal mapping, in other word there exists a constant matrix $A \in \mathbb{R}^{3 \times 3}$ and a constat vector $\mathbf{w} \in \mathbb{R}^{3}$ such that

$$
A^{T} \cdot A=I,
$$


and we have

$$
\mathbf{f}(\mathbf{x})=A \cdot \mathbf{x}+\mathbf{w} \quad \forall \mathbf{x} \in \mathbb{R}^{3}
$$

We give here the full proof, although, the result is well known.

Proof of Corollary 11.1. Consider two coordinate systems in $\mathbb{R}^{4}$, so that the change of coordinates from the first to the second coordinate system is given by:

$$
\left\{\begin{array}{l}
x^{\prime 0}=x^{0}, \\
x^{\prime 1}=f_{1}\left(x^{1}, x^{2}, x^{3}\right), \\
x^{\prime 2}=f_{2}\left(x^{1}, x^{2}, x^{3}\right), \\
x^{\prime 3}=f_{3}\left(x^{1}, x^{2}, x^{3}\right),
\end{array}\right.
$$

where $\left(f_{1}\left(x^{1}, x^{2}, x^{3}\right), f_{2}\left(x^{1}, x^{2}, x^{3}\right), f_{3}\left(x^{1}, x^{2}, x^{3}\right)\right):=\mathbf{f}\left(x^{1}, x^{2}, x^{3}\right)$. Next, consider a contravariant pseudo-metric $\left\{K^{m n}\right\}_{m, n=0,1,2,3}$ on $\mathbb{R}^{4}$ and the corresponding inverse covariant pseudo-metric $\left\{K_{m n}\right\}_{m, n=0,1,2,3}$, such that in the first coordinate system we have:

$$
\left\{\begin{array}{l}
K^{00}=1 \\
K^{0 j}=K^{j 0}=0 \quad \forall j=1,2,3 \\
K^{j m}=-\delta_{j m} \quad \forall j, m=1,2,3
\end{array}\right.
$$

at every point in $\mathbb{R}^{4}$, so that in the same coordinate system we have:

$$
\left\{\begin{array}{l}
K_{00}=1 \\
K_{0 j}=K_{j 0}=0 \quad \forall j=1,2,3 \\
K_{j m}=-\delta_{j m} \quad \forall j, m=1,2,3
\end{array}\right.
$$

at every point in $\mathbb{R}^{4}$. On the other hand, by (11.30) and (10.5) we have

$$
\begin{gathered}
K_{00}=K_{00}^{\prime} \\
K_{m 0}=K_{0 m}=\sum_{k=1}^{3} \frac{\partial f^{(k)}}{\partial x^{m}} K_{k 0}^{\prime}=\sum_{k=1}^{3} \frac{\partial f^{(k)}}{\partial x^{m}} K_{0 k}^{\prime} \quad \forall m=1,2,3 .
\end{gathered}
$$

and

$$
K_{m n}=\sum_{j=1}^{3} \sum_{k=1}^{3} \frac{\partial f^{(k)}}{\partial x^{m}} \frac{\partial f^{(j)}}{\partial x^{n}} K_{k j}^{\prime} \quad \forall m, n=1,2,3 .
$$

Therefore, using (11.27) and (11.32), by (11.33), (11.34) and (11.35) we deduce

$$
\begin{cases}K_{00}^{\prime}=1 & \\ K_{0 j}^{\prime}=K_{j 0}^{\prime}=0 & \forall j=1,2,3 \\ K_{j m}^{\prime}:=-\delta_{j m} & \forall j, m=1,2,3 .\end{cases}
$$


Then, by (11.32) and (11.36) we can apply Lemma 11.3 to deduce

$$
\frac{\partial^{2} f^{(j)}}{\partial x^{n} \partial x^{k}}\left(x^{1}, x^{2}, x^{3}\right)=0 \quad \forall\left(x^{1}, x^{2}, x^{3}\right) \in \mathbb{R}^{3} \quad \forall j, k, n=1,2,3 .
$$

Therefore, there exists a constant (independent on $\mathbf{x}$ ) matrix $A \in \mathbb{R}^{3 \times 3}$ such that

$$
A^{T} \cdot A=I
$$

and

$$
d_{\mathbf{x}} \mathbf{f}(\mathbf{x})=A \quad \forall x \in \mathbb{R}^{3} .
$$

So there exists a constat vector $\mathbf{w} \in \mathbb{R}^{3}$, such that

$$
\mathbf{f}(\mathbf{x})=A \cdot \mathbf{x}+\mathbf{w} \quad \forall x \in \mathbb{R}^{3}
$$

This completes the proof.

Lemma 11.4. Given an arbitrary $\left(w^{0}, w^{1}, w^{2}, w^{3}\right) \in \mathbb{R}^{4}$ such that

$$
\left(w^{0}\right)^{2}-|\mathbf{w}|^{2}=1
$$

where $\mathbf{w}:=\left(w^{1}, w^{2}, w^{3}\right) \in \mathbb{R}^{3}$, consider a matrix $\left\{\Lambda^{m n}\right\}_{0 \leq m, n \leq 3} \in \mathbb{R}^{4 \times 4}$, defined by

$$
\left\{\begin{array}{l}
\Lambda^{00}=\left(w^{0}\right)^{2}-1 \\
\Lambda^{j m}=\delta_{j m}+w^{j} w^{m} \quad \forall 1 \leq j, m \leq 3 \\
\Lambda^{0 j}=\Lambda^{j 0}=w^{0} w^{j} \quad \forall 1 \leq j \leq 3 .
\end{array}\right.
$$

Then the matrix $\left\{\Lambda^{m n}\right\}_{0 \leq m, n \leq 3}$ is degenerate and moreover, it has one vanishing and three positive eigenvalues.

Proof of Lemma 11.4. By (11.42) and (11.41) we have

$$
\left\{\begin{array}{l}
\Lambda^{00}=|\mathbf{w}|^{2} \\
\Lambda^{j m}=\delta_{j m}+w^{j} w^{m} \quad \forall 1 \leq j, m \leq 3 \\
\Lambda^{0 j}=\Lambda^{j 0}=w^{0} w^{j} \quad \forall 1 \leq j \leq 3,
\end{array}\right.
$$

Thus, in the case $\mathbf{w}=0$ we obviously have

$$
\left\{\begin{array}{l}
\Lambda^{00}=0 \\
\Lambda^{j m}=\delta_{j m} \quad \forall 1 \leq j, m \leq 3 \\
\Lambda^{0 j}=\Lambda^{j 0}=0 \quad \forall 1 \leq j \leq 3
\end{array}\right.
$$

and so, by (11.44) we infer that $\left\{\Lambda^{m n}\right\}_{0 \leq m, n \leq 3}$ is degenerate and moreover, it indeed has one vanishing and three positive eigenvalues. 
Next we assume from now that $\mathbf{w} \neq 0$. Furthermore, observe that by (11.42), for every $\left(z_{0}, z_{1}, z_{2}, z_{3}\right) \in \mathbb{R}^{4}$, with $\mathbf{z}:=\left(z_{1}, z_{2}, z_{3}\right) \in \mathbb{R}^{3}$, we have

$$
\sum_{j=0}^{3} \Lambda^{0 j} z_{j}=\Lambda^{00} z_{0}+\sum_{j=1}^{3} \Lambda^{0 j} z_{j}=\left(\left(w^{0}\right)^{2}-1\right) z_{0}+w^{0}(\mathbf{w} \cdot \mathbf{z})
$$

and

$$
\begin{aligned}
\sum_{j=0}^{3} \Lambda^{m j} z_{j}=\Lambda^{m 0} z_{0}+\sum_{j=1}^{3} \Lambda^{m j} z_{j}=z_{0} w^{0} & w^{m}+\sum_{j=1}^{3}\left(\delta_{j m}+w^{j} w^{m}\right) z_{j} \\
& =z_{0} w^{0} w^{m}+z_{m}+(\mathbf{w} \cdot \mathbf{z}) w^{m} \quad \forall m=1,2,3 .
\end{aligned}
$$

In other words,

$$
\left\{\begin{array}{l}
\sum_{j=0}^{3} \Lambda^{0 j} z_{j}=-z_{0}+\left(w^{0} z_{0}+\mathbf{w} \cdot \mathbf{z}\right) w^{0} \\
\sum_{j=0}^{3} \Lambda^{m j} z_{j}=z_{m}+\left(w^{0} z_{0}+\mathbf{w} \cdot \mathbf{z}\right) w^{m} \quad \forall m=1,2,3 .
\end{array}\right.
$$

Furthermore, assume that $\left(z_{0}, z_{1}, z_{2}, z_{3}\right) \in \mathbb{R}^{4}$, with $\mathbf{z}:=\left(z_{1}, z_{2}, z_{3}\right) \in \mathbb{R}^{3}$, satisfies

$$
\left\{\begin{array}{l}
z_{0}=\frac{1}{\lambda+1} w^{0} \\
\mathbf{z}=\frac{1}{\lambda-1} \mathbf{w}
\end{array}\right.
$$

where $\lambda$ solves

$$
\lambda\left(\lambda-\left(1+2|\mathbf{w}|^{2}\right)\right)=0
$$

or in other words, $\lambda$ satisfies

$$
\text { either } \quad \lambda=0 \quad \text { or } \quad \lambda=\left(2\left(w^{0}\right)^{2}-1\right)=\left(1+2|\mathbf{w}|^{2}\right)>1 \text {. }
$$

Then, in particular, by (11.48) we have

$$
\left(w^{0} z_{0}+\mathbf{w} \cdot \mathbf{z}\right)=\frac{1}{\lambda+1}\left(w^{0}\right)^{2}+\frac{1}{\lambda-1}|\mathbf{w}|^{2} .
$$

Then by (11.41) and (11.51) we have

$$
\left(w^{0} z_{0}+\mathbf{w} \cdot \mathbf{z}\right)=\left(\frac{1}{\lambda+1}+\frac{1}{\lambda-1}\right)\left(w^{0}\right)^{2}-\frac{1}{\lambda-1}=\frac{\lambda}{\lambda^{2}-1}\left(2\left(w^{0}\right)^{2}-1\right)-\frac{1}{\lambda^{2}-1} .
$$

If $\lambda=0$ then, by (11.52) we have clearly

$$
w^{0} z_{0}+\mathbf{w} \cdot \mathbf{z}=1 .
$$

On the other hand, if $\lambda=\left(1+2|\mathbf{w}|^{2}\right)=\left(2\left(w^{0}\right)^{2}-1\right)$ then by $(11.52)$ we also have

$$
\left(w^{0} z_{0}+\mathbf{w} \cdot \mathbf{z}\right)=\frac{\lambda}{\lambda^{2}-1} \lambda-\frac{1}{\lambda^{2}-1}=1 \text {. }
$$

Thus, if $\lambda$ solves (11.49) then in both cases $w^{0} z_{0}+\mathbf{w} \cdot \mathbf{z}=1$, and therefore, by (11.47) we deduce

$$
\left\{\begin{array}{l}
\sum_{j=0}^{3} \Lambda^{0 j} z_{j}=-z_{0}+w^{0} \\
\sum_{j=0}^{3} \Lambda^{m j} z_{j}=z_{m}+w^{m} \quad \forall m=1,2,3 .
\end{array}\right.
$$


However, by (11.48) we have

$$
\left\{\begin{array}{l}
w^{0}=(\lambda+1) z_{0} \\
\mathbf{w}=(\lambda-1) \mathbf{z}
\end{array}\right.
$$

Thus by (11.55) and (11.56) we deduce

$$
\left\{\begin{array}{l}
\sum_{j=0}^{3} \Lambda^{0 j} z_{j}=\lambda z_{0} \\
\sum_{j=0}^{3} \Lambda^{m j} z_{j}=\lambda z_{m} \quad \forall m=1,2,3 .
\end{array}\right.
$$

Thus, in the case where $\lambda$ solves (11.49) and $\left(z_{0}, z_{1}, z_{2}, z_{3}\right) \in \mathbb{R}^{4}$, with $\mathbf{z}:=\left(z_{1}, z_{2}, z_{3}\right) \in \mathbb{R}^{3}$, satisfies (11.48), by (11.57) we deduce that $\left(z_{0}, z_{1}, z_{2}, z_{3}\right)$ is an eigenvector of the matrix $\left\{\Lambda^{m n}\right\}_{0 \leq m, n \leq 3}$. So, $\lambda_{0}=0 \quad$ and $\quad \lambda_{1}=\left(1+2|\mathbf{w}|^{2}\right)>1 \quad$ are two eigenvalues of the matrix $\quad\left\{\Lambda^{m n}\right\}_{0 \leq m, n \leq 3}$.

Furthermore if $\lambda \in \mathbb{R}$ and $\left(z_{0}, z_{1}, z_{2}, z_{3}\right) \in \mathbb{R}^{4}$ with $\left(z_{1}, z_{2}, z_{3}\right):=\mathbf{z} \in \mathbb{R}^{3}$ satisfy

$$
\left\{\begin{array}{l}
\lambda=1 \\
\mathbf{z} \cdot \mathbf{w}=0 \\
z_{0}=0
\end{array}\right.
$$

then by (11.59) and (11.47) we have

$$
\left\{\begin{array}{l}
\sum_{j=0}^{3} \Lambda^{0 j} z_{j}=0=\lambda z_{0} \\
\sum_{j=0}^{3} \Lambda^{m j} z_{j}=z_{m}=\lambda z_{m} \quad \forall m=1,2,3 .
\end{array}\right.
$$

However, obviously there exists two orthogonal unit vectors $\mathbf{z}_{1} \in \mathbb{R}^{3}$ and $\mathbf{z}_{2} \in \mathbb{R}^{3}$ such that

$$
\mathbf{z}_{1} \cdot \mathbf{w}=\mathbf{z}_{2} \cdot \mathbf{w}=\mathbf{z}_{1} \cdot \mathbf{z}_{2}=0
$$

Therefore, in the case $\lambda=1$, if $\left(z_{0}, z_{1}, z_{2}, z_{3}\right) \in \mathbb{R}^{4}$ satisfies $z_{0}=0$ and either $\left(z_{1}, z_{2}, z_{3}\right)=\mathbf{z}_{1}$ or $\left(z_{1}, z_{2}, z_{3}\right)=\mathbf{z}_{2}$, then by (11.60) we deduce that in both cases $\left(z_{0}, z_{1}, z_{2}, z_{3}\right)$ is an eigenvector of of the matrix $\left\{\Lambda^{m n}\right\}_{0 \leq m, n \leq 3}$. So,

$$
\lambda_{2}=1 \quad \text { and } \quad \lambda_{3}=1 \quad \text { are two coinciding eigenvalues of the matrix } \quad\left\{\Lambda^{m n}\right\}_{0 \leq m, n \leq 3} .
$$

Therefore, by (11.58) and (11.61) we deduce that the matrix $\left\{\Lambda^{m n}\right\}_{0 \leq m, n \leq 3}$ is degenerate, and moreover it has one vanishing and three positive eigenvalues.

Lemma 11.5. Consider an arbitrary $\left(w^{0}, w^{1}, w^{2}, w^{3}\right) \in \mathbb{R}^{4}$ and consider a matrix $\left\{K^{m n}\right\}_{0 \leq m, n \leq 3} \in$ $\mathbb{R}^{4 \times 4}$, defined by:

$$
\left\{\begin{array}{l}
K^{00}=\left(w^{0}\right)^{2} \\
K^{j m}=-\delta_{j m}+w^{j} w^{m} \quad \forall 1 \leq j, m \leq 3 \\
K^{0 j}=K^{j 0}=w^{0} w^{j} \quad \forall 1 \leq j \leq 3 .
\end{array}\right.
$$


Then,

$$
\text { in the case } w^{0}=0 \text { we have } \operatorname{det}\left(\left\{K^{m n}\right\}_{0 \leq m, n \leq 3}\right)=0 .
$$

On the other hand, in the case $w^{0} \neq 0$ the matrix $\left\{K^{m n}\right\}_{0 \leq m, n \leq 3}$ is invertible, its reverse matrix $\left\{K_{m n}\right\}_{0 \leq m, n \leq 3} \in \mathbb{R}^{4 \times 4}$ is defined by the following:

$$
\left\{\begin{array}{l}
K_{00}=\frac{1}{\left(w^{0}\right)^{2}}-\frac{|\mathbf{w}|^{2}}{\left(w^{0}\right)^{2}} \\
K_{j m}=-\delta_{j m} \quad \forall 1 \leq j, m \leq 3 \\
K_{0 j}=K_{j 0}=\frac{w^{j}}{w^{0}} \quad \forall 1 \leq j \leq 3,
\end{array}\right.
$$

where $\mathbf{w}:=\left(w^{1}, w^{2}, w^{3}\right) \in \mathbb{R}^{3}$. Moreover, the matrix $\left\{K^{m n}\right\}_{0 \leq m, n \leq 3}$ necessary has one positive and three negative eigenvalues and

$$
\operatorname{det}\left(\left\{K^{m n}\right\}_{0 \leq m, n \leq 3}\right)=-\left(w^{0}\right)^{2} .
$$

Proof of Lemma 11.5. First of all, observe that in the case $w^{0}=0$ the first row of the matrix, given by (11.62), vanishes and thus we obviously deduce (11.63).

Next, assume from now that $w^{0} \neq 0$. Then, consider a matrix $\left\{K_{m n}\right\}_{0 \leq m, n \leq 3} \in \mathbb{R}^{4 \times 4}$ defined by (11.64). Thus, by (11.62) and (11.64) we obtain

$$
\begin{gathered}
\sum_{k=0}^{3} K_{0 k} K^{k 0}=K_{00} K^{00}+\sum_{k=1}^{3} K_{0 k} K^{k 0}=1-|\mathbf{w}|^{2}+|\mathbf{w}|^{2}=1, \\
\sum_{k=0}^{3} K_{m k} K^{k j}=K_{m 0} K^{0 j}+\sum_{k=1}^{3} K_{m k} K^{k j}=w^{m} w^{j}+\delta_{m j}-w^{m} w^{j}=\delta_{m j} \quad \forall 1 \leq m, j \leq 3,
\end{gathered}
$$

and

$$
\begin{gathered}
\sum_{k=0}^{3} K_{m k} K^{k 0}=K_{m 0} K^{00}+\sum_{k=1}^{3} K_{m k} K^{k 0}=w^{m} w^{0}-w^{m} w^{0}=0 \quad \forall 1 \leq m \leq 3, \\
\sum_{k=0}^{3} K_{0 k} K^{k j}=K_{00} K^{0 j}+\sum_{k=1}^{3} K_{0 k} K^{k j}=\frac{1}{w^{0}}\left(1-|\mathbf{w}|^{2}\right) w^{j}-\sum_{k=1}^{3} \frac{w^{k}}{w^{0}}\left(\delta_{k j}-w^{k} w^{j}\right)=0 \quad \forall 1 \leq j \leq 3 .
\end{gathered}
$$

So,

$$
\sum_{k=0}^{3} K^{m k} K_{k j}=\left\{\begin{array}{lll}
1 & \text { if } & m=j \\
0 & \text { if } & i \neq j
\end{array} \quad \forall m, j=0,1,2,3 .\right.
$$

Therefore, we deduce that $\left\{K_{m n}\right\}_{0 \leq m, n \leq 3}$ is an inverse matrix to $\left\{K^{m n}\right\}_{0 \leq m, n \leq 3} \in \mathbb{R}^{4 \times 4}$ and, in particular, in the case $w^{0} \neq 0$ matrix $\left\{K^{m n}\right\}_{0 \leq m, n \leq 3} \in \mathbb{R}^{4 \times 4}$ is invertible.

Next, in the case $\mathbf{w}:=\left(w^{1}, w^{2}, w^{3}\right)=0$ we are done, since then matrix $\left\{K^{m n}\right\}_{0 \leq m, n \leq 3} \in \mathbb{R}^{4 \times 4}$ obviously satisfies,

$$
\left\{\begin{array}{l}
K^{00}=\left(w^{0}\right)^{2} \\
K^{j m}=-\delta_{j m} \quad \forall 1 \leq j, m \leq 3 \\
K^{0 j}=K^{j 0}=0 \quad \forall 1 \leq j \leq 3,
\end{array}\right.
$$


and thus it has one positive eigenvalue $\lambda_{0}=\left(w^{0}\right)^{2}$, three negative eigenvalues $\lambda_{1}=\lambda_{2}=\lambda_{3}=-1$ and we have (11.65).

Thus, we assume from now that $w^{0} \neq 0$ and $\mathbf{w}:=\left(w^{1}, w^{2}, w^{3}\right) \neq 0$. Furthermore, observe that, by (11.62) for every $\left(z_{0}, z_{1}, z_{2}, z_{3}\right) \in \mathbb{R}^{4}$, with $\mathbf{z}:=\left(z_{1}, z_{2}, z_{3}\right) \in \mathbb{R}^{3}$, we have

$$
\sum_{j=0}^{3} K^{0 j} z_{j}=K^{00} z_{0}+\sum_{j=1}^{3} K^{0 j} z_{j}=\left(w^{0}\right)^{2} z_{0}+w^{0}(\mathbf{w} \cdot \mathbf{z})
$$

and

$$
\begin{aligned}
& \sum_{j=0}^{3} K^{m j} z_{j}=K^{m 0} z_{0}+\sum_{j=1}^{3} K^{m j} z_{j}=z_{0} w^{0} w^{m}+\sum_{j=1}^{3}\left(-\delta_{j m}+w^{j} w^{m}\right) z_{j} \\
&=z_{0} w^{0} w^{m}-z_{m}+(\mathbf{w} \cdot \mathbf{z}) w^{m} \quad \forall m=1,2,3 .
\end{aligned}
$$

In other words,

$$
\left\{\begin{array}{l}
\sum_{j=0}^{3} K^{0 j} z_{j}=\left(w^{0} z_{0}+\mathbf{w} \cdot \mathbf{z}\right) w^{0} \\
\sum_{j=0}^{3} K^{m j} z_{j}=-z_{m}+\left(w^{0} z_{0}+\mathbf{w} \cdot \mathbf{z}\right) w^{m} \quad \forall m=1,2,3 .
\end{array}\right.
$$

Next, if $\left(z_{0}, z_{1}, z_{2}, z_{3}\right) \in \mathbb{R}^{4}$, with $\mathbf{z}:=\left(z_{1}, z_{2}, z_{3}\right) \in \mathbb{R}^{3}$, satisfies

$$
\left\{\begin{array}{l}
\mathbf{z}=\frac{1}{\lambda+1} \mathbf{w} \\
z_{0}=\frac{1}{\lambda} w^{0}
\end{array}\right.
$$

where $\lambda \in \mathbb{R}$ is a solution of the following quadratic equation

$$
\lambda^{2}+\left(1-\left(w^{0}\right)^{2}-|\mathbf{w}|^{2}\right) \lambda-\left(w^{0}\right)^{2}=0
$$

(obviously that if $w^{0} \neq 0$ and $\mathbf{w} \neq 0$, then $\lambda \in\{-1,0\}$ does not satisfies (11.72)). Thus in particular, by (11.71) and (11.72) we have:

$$
w^{0} z_{0}+\mathbf{w} \cdot \mathbf{z}=\frac{1}{\lambda}\left|w^{0}\right|^{2}+\frac{1}{\lambda+1}|\mathbf{w}|^{2}=\frac{(\lambda+1)\left|w^{0}\right|^{2}+\lambda|\mathbf{w}|^{2}}{\lambda(\lambda+1)}=1 .
$$

Therefore, by (11.73) and (11.70) we deduce:

$$
\left\{\begin{array}{l}
\sum_{j=0}^{3} K^{0 j} z_{j}=w^{0} \\
\sum_{j=0}^{3} K^{m j} z_{j}=-z_{m}+w^{m} \quad \forall m=1,2,3
\end{array}\right.
$$

However, by (11.71) we have

$$
\left\{\begin{array}{l}
\mathbf{w}=(\lambda+1) \mathbf{z} \\
w^{0}=\lambda z_{0}
\end{array}\right.
$$

Therefore, by (11.75) and (11.74) we deduce:

$$
\sum_{j=0}^{3} K^{m j} z_{j}:=\lambda z_{m} \quad \forall m=0,1,2,3 .
$$


So, we obtain that, if $\lambda$ is a root of the quadratic equation (11.72) then, by (11.76) $\left(z_{0}, z_{1}, z_{2}, z_{3}\right) \in \mathbb{R}^{4}$, with $\mathbf{z}:=\left(z_{1}, z_{2}, z_{3}\right) \in \mathbb{R}^{3}$, given by (11.71), is an eigenvector of the matrix $\left\{K^{m n}\right\}_{0 \leq m, n \leq 3}$. In other words, every root of the quadratic equation (11.72) is an eigenvalue of the matrix $\left\{K^{m n}\right\}_{0 \leq m, n \leq 3}$. However, by Vieta's formulas the quadratic equation (11.72) has two distinct real roots $\lambda_{0}>0$ and $\lambda_{1}<0$ and $\lambda_{0} \lambda_{1}=-\left(w^{0}\right)^{2}$. Moreover, if $w^{0} \neq 0$ and $\mathbf{w} \neq 0$ then -1 does not satisfies (11.72)), and so,

$\lambda_{0}>0$ and $\quad-1 \neq \lambda_{1}<0$ are two eigenvalues of the matrix $\left\{K^{m n}\right\}_{0 \leq m, n \leq 3}$

$$
\text { and } \quad \lambda_{0} \lambda_{1}=-\left(w^{0}\right)^{2} \text {. }
$$

Furthermore, if $\lambda \in \mathbb{R}$ and $\left(z_{0}, z_{1}, z_{2}, z_{3}\right) \in \mathbb{R}^{4}$, with $\mathbf{z}:=\left(z_{1}, z_{2}, z_{3}\right) \in \mathbb{R}^{3}$, satisfy

$$
\left\{\begin{array}{l}
\lambda=-1 \\
\mathbf{z} \cdot \mathbf{w}=0 \\
z_{0}=0
\end{array}\right.
$$

then by (11.78) and (11.70) we have

$$
\left\{\begin{array}{l}
\sum_{j=0}^{3} K^{0 j} z_{j}=0=\lambda z_{0} \\
\sum_{j=0}^{3} K^{m j} z_{j}=-z_{m}=\lambda z_{m} \quad \forall m=1,2,3 .
\end{array}\right.
$$

However, obviously there exists two orthogonal unit vectors $\mathbf{z}_{1} \in \mathbb{R}^{3}$ and $\mathbf{z}_{2} \in \mathbb{R}^{3}$ such that

$$
\mathbf{z}_{1} \cdot \mathbf{w}=\mathbf{z}_{2} \cdot \mathbf{w}=\mathbf{z}_{1} \cdot \mathbf{z}_{2}=0 .
$$

Therefore, in the case $\lambda=-1$, if $\left(z_{0}, z_{1}, z_{2}, z_{3}\right) \in \mathbb{R}^{4}$ satisfies $z_{0}=0$ and either $\left(z_{1}, z_{2}, z_{3}\right)=\mathbf{z}_{1}$ or $\left(z_{1}, z_{2}, z_{3}\right)=\mathbf{z}_{2}$, then by (11.79) we deduce that in both cases $\left(z_{0}, z_{1}, z_{2}, z_{3}\right)$ is an eigenvector of of the matrix $\left\{K^{m n}\right\}_{0 \leq m, n \leq 3}$. So,

$$
\lambda_{2}=-1 \quad \text { and } \quad \lambda_{3}=-1 \text { are two coinciding eigenvalues of the matrix }\left\{K^{m n}\right\}_{0 \leq m, n \leq 3} .
$$

Therefore, by (11.77) and (11.80) we deduce that the matrix $\left\{K^{m n}\right\}_{0 \leq m, n \leq 3}$ has one positive and three negative eigenvalues and moreover,

$$
\lambda_{0} \lambda_{1} \lambda_{2} \lambda_{3}=-\left(w^{0}\right)^{2} .
$$

However, it is well known from the Linear Algebra that

$$
\lambda_{0} \lambda_{1} \lambda_{2} \lambda_{3}=\operatorname{det}\left(\left\{K^{m n}\right\}_{0 \leq m, n \leq 3}\right)
$$

and thus, by (11.81) we finally deduce (11.65). 
Lemma 11.6. Let $\left\{\Lambda^{m n}\right\}_{0 \leq m, n \leq 3} \in \mathbb{R}^{4 \times 4}$ be a degenerate symmetric matrix, given by

$$
\left\{\begin{array}{l}
\Lambda^{00}=0 \\
\Lambda^{j m}=\delta_{j m} \quad \forall 1 \leq j, m \leq 3 \\
\Lambda^{0 j}=\Lambda^{j 0}=0 \quad \forall 1 \leq j \leq 3,
\end{array}\right.
$$

with

$$
\begin{cases}\delta_{j j}=1 & \forall j=1,2,3 \\ \delta_{j m}=0 & \forall j \neq m=1,2,3,\end{cases}
$$

and let $\left(w^{0}, w^{1}, w^{2}, w^{3}\right) \in \mathbb{R}^{4}$ be such that

$$
w^{0} \neq 0
$$

Then, there exists a non-degenerate matrix $\left\{A_{m n}\right\}_{0 \leq m, n \leq 3} \in \mathbb{R}^{4 \times 4}$, such that $\operatorname{det}\left(\left\{A_{m n}\right\}_{0 \leq m, n \leq 3}\right) \neq$ 0 , and if we consider a matrix $\left\{\Lambda^{\prime m n}\right\}_{0 \leq m, n \leq 3} \in \mathbb{R}^{4 \times 4}$, defined by

$$
\Lambda^{\prime m n}:=\sum_{j=0}^{3} \sum_{k=0}^{3} A_{m k} A_{n j} \Lambda^{k j} \quad \forall m, n=0,1,2,3,
$$

and a vector $\left(w^{\prime 0}, w^{\prime 1}, w^{\prime 2}, w^{\prime 3}\right) \in \mathbb{R}^{4}$, defined by

$$
w^{\prime j}:=\sum_{k=0}^{3} A_{j k} w^{k} \quad \forall j=0,1,2,3,
$$

then we have:

$$
\left\{\begin{array}{l}
\Lambda^{\prime 00}=0 \\
\Lambda^{\prime j m}=\delta_{j m} \quad \forall 1 \leq j, m \leq 3 \\
\Lambda^{\prime 0 j}=\Lambda^{\prime j 0}=0 \quad \forall 1 \leq j \leq 3,
\end{array}\right.
$$

and

$$
\left(w^{\prime 0}, w^{\prime 1}, w^{\prime 2}, w^{\prime 3}\right)=(1,0,0,0)
$$

Proof of Lemma 11.6. Consider a a non-degenerate matrix $\left\{A_{m n}\right\}_{0 \leq m, n \leq 3} \in \mathbb{R}^{4 \times 4}$ defined by:

$$
\left\{\begin{array}{l}
A_{00}=\frac{1}{w^{0}} \\
A_{j m}=\delta_{j m} \quad \forall 1 \leq j, m \leq 3 \\
A_{j 0}=-\frac{w^{j}}{w^{0}} \quad \forall 1 \leq j \leq 3 \\
A_{0 j}=0 \quad \forall 1 \leq j \leq 3 .
\end{array}\right.
$$

Then, by (11.86) and (11.89) we obtain

$$
w^{\prime j}=A_{j 0} w^{0}+\sum_{k=1}^{3} A_{j k} w^{k}=\left(-\frac{w^{j}}{w^{0}}\right) w^{0}+\sum_{k=1}^{3} \delta_{j k} w^{k}=-w^{j}+w^{j}=0 \quad \forall j=1,2,3,
$$


and

$$
w^{\prime 0}=A_{00} w^{0}+\sum_{k=1}^{3} A_{0 k} w^{k}=\left(\frac{1}{w^{0}}\right) w^{0}+0=1 .
$$

So we deduce (11.88). On the other hand, by (11.85) and (11.82) we deuce:

$$
\Lambda^{\prime m n}=\sum_{j=0}^{3} \sum_{k=0}^{3} A_{m k} A_{n j} \Lambda^{k j}=\sum_{j=1}^{3} \sum_{k=1}^{3} A_{m k} A_{n j} \delta_{k j}=\sum_{j=1}^{3} A_{m j} A_{n j} \quad \forall m, n=0,1,2,3 .
$$

Therefore, by (11.92) and (11.89) we infer

$$
\begin{gathered}
\Lambda^{\prime 00}=\sum_{j=1}^{3} A_{0 j}^{2}=0, \\
\Lambda^{\prime m 0}=\Lambda^{\prime 0 m}=\sum_{j=1}^{3} A_{m j} A_{0 j}=0 \quad \forall m=1,2,3,
\end{gathered}
$$

and

$$
\Lambda^{\prime m n}=\sum_{j=1}^{3} A_{m j} A_{n j}=\sum_{j=1}^{3} \delta_{m j} \delta_{n j}=\delta_{m n} \quad \forall m, n=1,2,3 .
$$

So, by (11.93), (11.94) and (11.95) we also deduce (11.87). This completes the proof.

Lemma 11.7. Let $\left\{K^{m n}\right\}_{0 \leq m, n \leq 3} \in \mathbb{R}^{4 \times 4}$ be a non-degenerate symmetric matrix, given by

$$
\left\{\begin{array}{l}
K^{00}=1 \\
K^{j m}=-\delta_{j m} \quad \forall 1 \leq j, m \leq 3 \\
K^{0 j}=K^{j 0}=0 \quad \forall 1 \leq j \leq 3,
\end{array}\right.
$$

and let $\left(w^{0}, w^{1}, w^{2}, w^{3}\right) \in \mathbb{R}^{4}$ be such that the real number $M$, given by

$$
M:=\left|w^{0}\right|^{2}-\sum_{j=1}^{3}\left|w^{j}\right|^{2}
$$

satisfies

$$
M>0
$$

Then, there exists a non-degenerate matrix $\left\{A_{m n}\right\}_{0 \leq m, n \leq 3} \in \mathbb{R}^{4 \times 4}$, such that $\operatorname{det}\left(\left\{A_{m n}\right\}_{0 \leq m, n \leq 3}\right) \neq$ 0 , and if we consider a matrix $\left\{K^{\prime m n}\right\}_{0 \leq m, n \leq 3} \in \mathbb{R}^{4 \times 4}$, defined by

$$
K^{\prime m n}:=\sum_{j=0}^{3} \sum_{k=0}^{3} A_{m k} A_{n j} K^{k j} \quad \forall m, n=0,1,2,3,
$$

and a vector $\left(w^{\prime 0}, w^{\prime 1}, w^{\prime 2}, w^{\prime 3}\right) \in \mathbb{R}^{4}$, defined by

$$
w^{\prime j}:=\sum_{k=0}^{3} A_{j k} w^{k} \quad \forall j=0,1,2,3,
$$

then we have:

$$
\left\{\begin{array}{l}
K^{\prime 00}=1 \\
K^{\prime j m}=-\delta_{j m} \quad \forall 1 \leq j, m \leq 3 \\
K^{\prime 0 j}=K^{\prime j 0}=0 \quad \forall 1 \leq j \leq 3
\end{array}\right.
$$


and

$$
\left(w^{\prime 0}, w^{\prime 1}, w^{\prime 2}, w^{\prime 3}\right)=(\sqrt{M}, 0,0,0)
$$

Proof of Lemma 11.7. Without any loss of generality we may assume $M=1$ in (11.97), otherwise we just replace $\left(w^{0}, w^{1}, w^{2}, w^{3}\right)$ by $\left(\frac{w^{0}}{\sqrt{M}}, \frac{w^{1}}{\sqrt{M}}, \frac{w^{2}}{\sqrt{M}}, \frac{w^{3}}{\sqrt{M}}\right)$. So consider from now that $M=1$. In other words, we have

$$
\left|w^{0}\right|^{2}-\sum_{j=1}^{3}\left|w^{j}\right|^{2}=1
$$

Then, by (11.103), using Lemma 11.4 we deduce that a matrix $\left\{\Lambda^{m n}\right\}_{0 \leq m, n \leq 3} \in \mathbb{R}^{4 \times 4}$, defined by

$$
\left\{\begin{array}{l}
\Lambda^{00}=\left(w^{0}\right)^{2}-1 \\
\Lambda^{j m}=\delta_{j m}+w^{j} w^{m} \quad \forall 1 \leq j, m \leq 3 \\
\Lambda^{0 j}=\Lambda^{j 0}=w^{0} w^{j} \quad \forall 1 \leq j \leq 3,
\end{array}\right.
$$

has one vanishing and three positive eigenvalues. Therefor, by the Sylvester's law of inertia, there exists a non-degenerate matrix $\left\{B_{m n}\right\}_{0 \leq m, n \leq 3} \in \mathbb{R}^{4 \times 4}$, such that $\operatorname{det}\left(\left\{B_{m n}\right\}_{0 \leq m, n \leq 3}\right) \neq 0$, and if we consider a matrix $\left\{\Lambda^{\prime \prime m n}\right\}_{0 \leq m, n \leq 3} \in \mathbb{R}^{4 \times 4}$, defined by

$$
\Lambda^{\prime \prime m n}:=\sum_{j=0}^{3} \sum_{k=0}^{3} B_{m k} B_{n j} \Lambda^{k j} \quad \forall m, n=0,1,2,3,
$$

then we have

$$
\left\{\begin{array}{l}
\Lambda^{\prime \prime 00}=0 \\
\Lambda^{\prime \prime j m}=\delta_{j m} \quad \forall 1 \leq j, m \leq 3 \\
\Lambda^{\prime \prime 0 j}=\Lambda^{\prime \prime j 0}=0 \quad \forall 1 \leq j \leq 3 .
\end{array}\right.
$$

However, by (11.101) and (11.104) we have

$$
K^{m n}=w^{m} w^{n}-\Lambda^{m n}: \quad \forall m, n=0,1,2,3 .
$$

Thus, if we consider a vector $\left(w^{\prime \prime 0}, w^{\prime \prime 1}, w^{\prime \prime 2}, w^{\prime \prime 3}\right) \in \mathbb{R}^{4}$, defined by

$$
w^{\prime \prime j}:=\sum_{k=0}^{3} B_{j k} w^{k} \quad \forall j=0,1,2,3,
$$

and a matrix $\left\{K^{\prime \prime m n}\right\}_{0 \leq m, n \leq 3} \in \mathbb{R}^{4 \times 4}$, defined by

$$
K^{\prime \prime m n}:=\sum_{j=0}^{3} \sum_{k=0}^{3} B_{m k} B_{n j} K^{k j} \quad \forall m, n=0,1,2,3,
$$

then, by (11.107) and (11.109) we deduce

$$
K^{\prime \prime m n}=w^{\prime \prime m} w^{\prime \prime n}-\Lambda^{\prime \prime m n}: \quad \forall m, n=0,1,2,3 .
$$


Then, by (11.110) and (11.106) we obtain

$$
\begin{cases}K^{\prime \prime 00}=\left(w^{\prime \prime 0}\right)^{2} & \\ K^{\prime \prime j m}=w^{\prime \prime j} w^{\prime \prime m}-\delta_{j m} & \forall 1 \leq j, m \leq 3 \\ K^{\prime \prime 0 j}=K^{\prime \prime j 0}=w^{\prime \prime 0} w^{\prime \prime j} & \forall 1 \leq j \leq 3 .\end{cases}
$$

In particular, since the matrix $\left\{K^{\prime \prime m n}\right\}_{0 \leq m, n \leq 3}$ is non-degenerate (follows by the fact that $\left\{K^{m n}\right\}_{0 \leq m, n \leq 3}$ is non-degenerate), we deduce from (11.111) that we necessary have

$$
w^{\prime \prime 0} \neq 0
$$

Therefore, we can apply Lemma 11.6 to deduce that, there exists a non-degenerate matrix $\left\{A_{m n}^{\prime}\right\}_{0 \leq m, n \leq 3} \in \mathbb{R}^{4 \times 4}$, such that $\operatorname{det}\left(\left\{A_{m n}^{\prime}\right\}_{0 \leq m, n \leq 3}\right) \neq 0$, and if we consider a matrix $\left\{\Lambda^{\prime m n}\right\}_{0 \leq m, n \leq 3} \in \mathbb{R}^{4 \times 4}$, defined by

$$
\Lambda^{\prime m n}:=\sum_{j=0}^{3} \sum_{k=0}^{3} A_{m k}^{\prime} A_{n j}^{\prime} \Lambda^{\prime \prime k j} \quad \forall m, n=0,1,2,3,
$$

and a vector $\left(w^{\prime 0}, w^{\prime 1}, w^{\prime 2}, w^{\prime 3}\right) \in \mathbb{R}^{4}$, defined by

$$
w^{\prime j}:=\sum_{k=0}^{3} A_{j k}^{\prime} w^{\prime \prime k} \quad \forall j=0,1,2,3,
$$

then we have:

$$
\left\{\begin{array}{l}
\Lambda^{\prime 00}=0 \\
\Lambda^{\prime j m}=\delta_{j m} \quad \forall 1 \leq j, m \leq 3 \\
\Lambda^{\prime 0 j}=\Lambda^{\prime j 0}=0 \quad \forall 1 \leq j \leq 3
\end{array}\right.
$$

and

$$
\left(w^{\prime 0}, w^{1}, w^{\prime 2}, w^{\prime 3}\right)=(1,0,0,0)
$$

Therefore, considering a non-degenerate matrix $\left\{A_{m n}\right\}_{0 \leq m, n \leq 3} \in \mathbb{R}^{4 \times 4}$, defined by

$$
A_{m n}:=\sum_{k=0}^{3} A_{m k}^{\prime} B_{k n} \quad \forall j=0,1,2,3,
$$

we obviously deduce, $\operatorname{det}\left(\left\{A_{m n}\right\}_{0 \leq m, n \leq 3}\right) \neq 0$ and moreover, by (11.105) and (11.113) we have

$$
\Lambda^{\prime m n}:=\sum_{j=0}^{3} \sum_{k=0}^{3} A_{m k} A_{n j} \Lambda^{k j} \quad \forall m, n=0,1,2,3,
$$

and by (11.108) and (11.114) we have

$$
w^{\prime j}:=\sum_{k=0}^{3} A_{j k} w^{k} \quad \forall j=0,1,2,3 .
$$

Thus, if we consider a matrix $\left\{K^{\prime m n}\right\}_{0 \leq m, n \leq 3} \in \mathbb{R}^{4 \times 4}$, defined by

$$
K^{\prime m n}:=\sum_{j=0}^{3} \sum_{k=0}^{3} A_{m k} A_{n j} K^{k j} \quad \forall m, n=0,1,2,3,
$$


then, by (11.118), (11.119) and (11.107) we deduce

$$
K^{\prime m n}=w^{\prime m} w^{\prime n}-\Lambda^{\prime \prime m n} \quad \forall m, n=0,1,2,3 .
$$

Finally, inserting (11.115) and (11.116) into (11.121) gives

$$
\left\{\begin{array}{l}
K^{\prime 00}=1 \\
K^{\prime j m}=-\delta_{j m} \quad \forall 1 \leq j, m \leq 3 \\
K^{\prime 0 j}=K^{\prime j 0}=0 \quad \forall 1 \leq j \leq 3
\end{array}\right.
$$

and

$$
\left(w^{\prime 0}, w^{\prime 1}, w^{\prime 2}, w^{\prime 3}\right)=(1,0,0,0)
$$

where $\left\{K^{\prime m n}\right\}_{0 \leq m, n \leq 3}$ is given by $(11.120)$ and $\left(w^{\prime 0}, w^{\prime 1}, w^{\prime 2}, w^{\prime 3}\right)$ is given by (11.119) with $\operatorname{det}\left(\left\{A_{m n}\right\}_{0 \leq m, n \leq 3}\right) \neq 0$. This completes the proof.

\section{References}

[1] C. Duval, On Galilean isometries, Class. Quantum Grav. 10, 2217 (1993), doi:10.1088/02649381/10/11/006, [arXiv:0903.1641].

[2] C. Duval, G. Burdet, H. P. Künzle and M. Perrin, Bargmann structures and Newton-Cartan theory, Phys. Rev. D 31, 1841 (1985), doi:10.1103/PhysRevD.31.1841.

[3] C. Duval and P. A. Horváthy, Non-relativistic conformal symmetries and Newton-Cartan structures, J. Phys. A: Math. Theor. 42, 465206 (2009), doi:10.1088/1751- 8113/42/46/465206, arXiv:0904.0531.

[4] C. Duval and H. P. Künzle, Minimal gravitational coupling in the Newtonian theory and the covariant Schrödinger equation, Gen. Relat. Gravit. 16, 333 (1984), doi:10.1007/BF00762191.

[5] K. Jensen, On the coupling of Galilean-invariant field theories to curved spacetime, SciPost Phys. 5, 011 (2018).

[6] H. P. Künzle, Galilei and lorentz structures on space-time : comparison of the corresponding geometry and physics, Ann. Inst. H. Poincaré Phys. Théor. 17, 337 (1972).

[7] L.D. Landau, E.M. Lifshitz, The Classical Theory of Fields Vol. 2 in Course of Theoretical Physics Book (4rd ed.) (1975).

[8] H. Minkowski, Die Grundgleichungen für die elektromagnetischen Vorgänge in bewegten Körpern, Nachr. Ges. Wiss. Göttn Math.-Phys. Kl. 53 (1908); Reprinted in Math. Ann. 68, 472 (1910). 
[9] S. Weinberg, Gravitation and Cosmology: Principles and Applications of the General Theory of Relativity, 1972, Wiley 